NOTE TO USERS

This reproduction is the best copy available.

UMI 


\title{
Pattern Recognition of Functional Neuroimage Data of the Human Sensorimotor System After Stroke
}

\author{
by \\ Camille Gómez-Laberge, M.A.Sc., B.Eng., B.Sc.
}

A thesis submitted to the Faculty of Graduate Studies and Research in partial fulfilment of the requirements for the degree of Doctor of Philosophy in Electrical and Computer Engineering

Neuroscience Program, Ottawa Hospital Research Institute

Ottawa-Carleton Institute for Biomedical Engineering Department of Systems and Computer Engineering

Carleton University

Ottawa, Ontario, Canada, K1S 5B6

June 2010

(C) Camille Gómez-Laberge 


\section{Library and Archives} Canada

Published Heritage

Branch

395 Wellington Street Ottawa ON K1A ON4 Canada
Bibliothèque et

Archives Canada

Direction du

Patrimoine de l'édition

395 , rue Wellington

Ottawa ON K1A ON4

Canada
Your file Votre référence

ISBN: 978-0-494-67877-0

Ourfile Notre reférence

ISBN: 978-0-494-67877-0
NOTICE:

The author has granted a nonexclusive license allowing Library and Archives Canada to reproduce, publish, archive, preserve, conserve, communicate to the public by telecommunication or on the Internet, loan, distribute and sell theses worldwide, for commercial or noncommercial purposes, in microform, paper, electronic and/or any other formats.

The author retains copyright ownership and moral rights in this thesis. Neither the thesis nor substantial extracts from it may be printed or otherwise reproduced without the author's permission.
AVIS:

L'auteur a accordé une licence non exclusive permettant à la Bibliothèque et Archives Canada de reproduire, publier, archiver, sauvegarder, conserver, transmettre au public par télécommunication ou par l'Internet, prêter, distribuer et vendre des thèses partout dans le monde, à des fins commerciales ou autres, sur support microforme, papier, électronique et/ou autres formats.

L'auteur conserve la propriété du droit d'auteur et des droits moraux qui protège cette thèse. $\mathrm{Ni}$ la thèse ni des extraits substantiels de celle-ci ne doivent être imprimés ou autrement reproduits sans son autorisation.
In compliance with the Canadian Privacy Act some supporting forms may have been removed from this thesis.

While these forms may be included in the document page count, their removal does not represent any loss of content from the thesis.
Conformément à la loi canadienne sur la protection de la vie privée, quelques formulaires secondaires ont été enlevés de cette thèse.

Bien que ces formulaires aient inclus dans la pagination, il n'y aura aucun contenu manquant. 
The undersigned recommend to

the Faculty of Graduate Studies and Research acceptance of the thesis

\title{
Pattern Recognition of Functional Neuroimage Data of the Human Sensorimotor System After Stroke
}

\author{
submitted by \\ Camille Gómez-Laberge, M.A.Sc., B.Eng., B.Sc.
}

in partial fulfilment of the requirements for the degree of Doctor of Philosophy in Electrical and Computer Engineering

Chair, Howard M. Schwartz, Ph.D., P.Eng. Department of Systems and Computer Engineering

Thesis Supervisor, Andy Adler, Ph.D., P.Eng. Department of Systems and Computer Engineering

Thesis Supervisor, Matthew J. Hogan, M.D., Ph.D., F.R.C.P.C. Neuroscience Program, Ottawa Hospital Research Institute Faculty of Medicine, University of Ottawa

External Examiner, G. Bruce Pike, Ph.D. McConnell Brain Imaging Centre, Montreal Neurological Institute Faculty of Medicine, McGill University

Carleton University

May 2010 
For Tanya 


\section{Abstract}

This thesis contributes to the study of the regulatory mechanisms of the cerebral circulation and its dysfunction in disease. Novel analytical methods are studied for the characterisation of the neurovascular mechanisms measured by functional magnetic resonance imaging of humans having suffered ischemic stroke.

Functional neuroimage data are acquired from consenting healthy volunteers and ischemic stroke patients with motor deficits who participated in an event-related visual feedback controlled hand motor task. The neuroscience and stroke literature provides the basis for this research on analytical methods for i) exploratory pattern recognition of the cerebrovascular responses to sensorimotor tasks, ii) the characterisation of their space-time structure, and iii) a Bayesian hierarchical model for evaluating their statistical significance for intersubject comparison. Ultimately, these methods aim to identify and distinguish between distinct response signals, thereby making them amenable to expert visual inspection and interpretation.

The results from the trials presented here show that the method is capable of differentiating between the response characteristics from the healthy and stroke patient groups. In the months following ischemic stroke, an abnormal increase in the number of distinct response signals occurs, exhibiting a dispersed spatial pattern and diminished temporal relationship to the motor task that is either extensively delayed, or abnormally early and anti-correlated. The most likely explanation is that these widely dispersed response signals appearing throughout the cerebral cortex of the stroke patients are no longer coupled to colocalised neural activity. According to this premise, our results suggest that the regulatory mechanisms of the cerebrovascular system can remain compromised in the months following ischemic stroke.

In summary, direct evidence is provided here supporting the application of exploratory pattern recognition and Bayesian hierarchical analysis of neuroimage data acquired during the study of ischemic stroke. Specifically, the aforementioned analytical methods are shown to be suitable for monitoring the spatiotemporal characteristics of the neurovascular response to sensorimotor stimuli. Its implementation in longitudinal trials would provide key information for the evaluation of stroke rehabilitation programmes and for the assessment of individual patient recovery. 


\section{Acknowledgements}

My utmost gratitude and appreciation is extended to my supervisors Dr. Matthew J. Hogan in the Neuroscience Program at the Ottawa Hospital Research Institute, and Dr. Andy Adler in the Faculty of Engineering at Carleton University. I am obliged for their judicious advice and criticism, steady encouragement and availability, and generous financial support during my doctoral training.

I am also indebted to my collaborators at The Ottawa Hospital: Dr. Ian Cameron and Dr. Greg Cron in the Diagnostic Imaging Department, Dr. Thanh Nguyen in the Radiology Division, as well as Dr. Andra Smith in the Psychology Department at the University of Ottawa. Their ability, resourcefulness, and dedication to the experimental work was instrumental to its success.

A fundamental part of my doctoral research experience revolves around my fellowship in the Neuroscience Program at the Ottawa Hospital Research Institute. I will always be grateful to the Neuroscience Program's faculty and students who, with exemplary dedication and talent, taught me about the marvels of neuroscience while always making me feel at home. I sincerely thank the Program director Dr. Antoine Hakim, and faculty members Dr. Charlie Thompson and Dr. Michael Schlossmacher for their mentorship and support.

I could never express enough gratitude for the unconditional encouragement and enthusiasm from my loving parents, family, and friends. Finally, I dedicate this thesis to my dearest love, Tanya. Her beauty bestows on me the greatest proof of nature's splendour.

Camille Gómez-Laberge

March, 2010

Ottawa, Canada 


\section{Contents}

Abstract $\quad$ iv

Acknowledgements $\quad$ v

Contents vi vi vis vis

List of Tables $\quad x$

List of Figures $\quad$ xi

List of Acronymns xix

1 Human Neurophysiology at the System Level 1

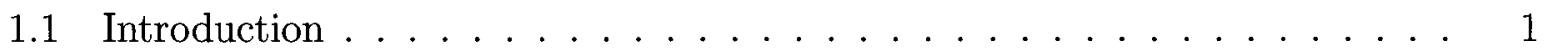

1.2 General neuroanatomy and physiology $\ldots \ldots \ldots \ldots$

1.3 Toward a theory of computational neuroscience $\ldots \ldots \ldots 7$

1.4 Gathering empirical evidence of brain function . . . . . . . . . . . . 12

1.4.1 Direct cortical recordings . . . . . . . . . . . . . . . . . 13

1.4 .2 Electromagnetic field recordings . . . . . . . . . . . . . . . 13

1.4 .3 Metabolic and vascular imaging . . . . . . . . . . . 14

1.4.4 Blood oxygenation imaging . . . . . . . . . . . . 15

1.5 Analytical methods for neuroimage data $\ldots \ldots \ldots 16$

1.5 .1 Pattern recognition $\ldots \ldots \ldots \ldots \ldots \ldots$ 
1.5.2 Parametric modelling . . . . . . . . . . . . . . . . 18

1.5.3 Statistical inference . . . . . . . . . . . . . . . . . . 19

1.6 Focus and summary of the thesis $\ldots \ldots \ldots \ldots$

$\begin{array}{llr}2 & \text { The Sensorimotor System } & 21\end{array}$

2.1 Introduction . . . . . . . . . . . . . . . 21

2.2 Functional anatomy of the sensorimotor system $\ldots \ldots \ldots . \ldots 21$

2.2 .1 The sensorimotor cortex $\ldots \ldots \ldots \ldots \ldots \ldots$

2.2.2 The basal ganglia and cerebellum . . . . . . . . . . . 24

2.3 The neurovascular unit $\ldots \ldots \ldots \ldots \ldots \ldots$

2.4 The effects of ischemic stroke . . . . . . . . . . . . . . 29

2.5 Imaging stroke recovery with fMRI . . . . . . . . . . . . . . 32

2.6 Discussion . . . . . . . . . . . . . . . . . . . 34

3 Data Acquisition I: Experiment Design \& Imaging 36

3.1 Introduction . . . . . . . . . . . . . . . . . . . . . . 36

3.2 Experimental design $\ldots \ldots \ldots \ldots \ldots \ldots$

3.3 Neuroimaging data . . . . . . . . . . . . . . . . . . . 41

3.4 Participation of normal and stroke subjects . . . . . . . . . . . . 44

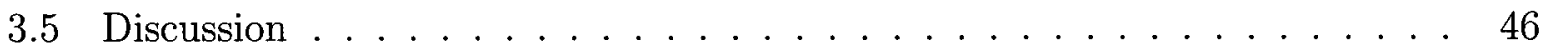

4 Data Acquisition II: Preliminary Analysis 48

4.1 Introduction . . . . . . . . . . . . . . . . . . . 48

4.2 Mean and autocorrelation function estimation . . . . . . . . . . 49

4.3 Physiological BOLD signal characterisation . . . . . . . . . . . 54

4.4 Data simulations . . . . . . . . . . . . . . . . . . . 58

4.5 Discussion . . . . . . . . . . . . . . . . . . . 61 
5.1 Introduction . . . . . . . . . . . . . . . . 64

5.2 Fuzzy set theory and partitions $\ldots \ldots \ldots \ldots 6 \ldots$

5.3 Fuzzy $k$-means on time sequence data . . . . . . . . . . . 70

5.4 Partition entropy minimisation $\ldots \ldots \ldots \ldots \ldots$

5.5 Implementation and results $\ldots \ldots \ldots \ldots \ldots$

5.6 Discussion . . . . . . . . . . . . . . . . . . 83

6 Analysis II: Space-Time Structure of BOLD Signal 86

6.1 Introduction . . . . . . . . . . . . . . . . . 86

6.2 Space characterisation: voxel cluster contiguity . . . . . . . . . . . 87

6.3 Time characterisation: causal cross-correlation . . . . . . . . . . . . . 92

6.4 Results . . . . . . . . . . . . . . . . . . . . . 94

6.5 Discussion . . . . . . . . . . . . . . . . 97

7 Analysis III: Bayesian Hierarchical Model 99

7.1 Introduction . . . . . . . . . . . . . . . . . . 99

7.2 Empirical inspection of clustered data . . . . . . . . . . . . . 101

7.3 Bayesian hierarchical model . . . . . . . . . . . . . . . . 103

$7.3 .1 \quad$ Joint probability distribution . . . . . . . . . . . . . . 104

7.3.2 The likelihood, population, and prior distributions . . . . . . . . 105

7.4 Gibbs sampling of the posterior density . . . . . . . . . . . 106

7.5 Results . . . . . . . . . . . . . . . . 111

7.6 Discussion . . . . . . . . . . . . . . . . . . 114

8 Imaging Motor Deficits After Stroke $\quad 117$

8.1 Introduction . . . . . . . . . . . . . . . . 117

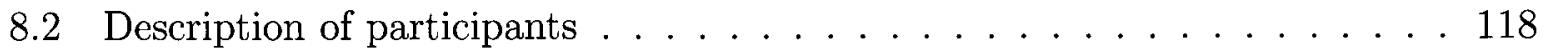

$8.2 .1 \quad$ Normal subjects . . . . . . . . . . . . . . . . . . 118 
8.2 .2 Stroke patients . . . . . . . . . . . . . . . 119

8.3 Results . . . . . . . . . . . . . . . . . . . . 123

8.3 .1 Motor response data . . . . . . . . . . . . . 123

8.3 .2 Neuroimage data . . . . . . . . . . . . . . 125

8.3.3 Bayesian hierarchical model . . . . . . . . . . . . . 127

8.3.4 Space-time structure of selected clusters . . . . . . . . . 129

8.3.5 Identified sensorimotor-related brain regions . . . . . . . . . . . 132

8.4 Discussion . . . . . . . . . . . . . . . . . . . . 141

9 Conclusion $\quad 144$

9.1 Introduction . . . . . . . . . . . . . . . . 144

9.2 Summary and critique of the proposed methods . . . . . . . . . . 145

9.3 Summary of the neuroimage stroke trial results . . . . . . . . . 147

9.4 General conclusion and future directions $\ldots \ldots \ldots$

$\begin{array}{lr}\text { Bibliography } & 150\end{array}$

$\begin{array}{ll}\text { A Ethics and Experimental Protocol } & 162\end{array}$

A.1 Research ethics board letter of approval . . . . . . . . . . . . . . 162

A.2 Protocol involving human subject particiaption $\ldots \ldots \ldots 165$

A.3 Consent form and supporting documents . . . . . . . . . . . . 184 


\section{List of Tables}

8.1 Participants in the normal group. Subject 2 declared that he is taking medication to control his hypertension. . . . . . . . . . . . . . . 119

8.2 Participants in the ischemic stroke group. . . . . . . . . . . 120

8.3 Summary of stroke group motor responses. The response strength is qualitatively described by $=$ when it was close to the target strength of $25 \%$ MVC, and by $\Uparrow$ or $\Downarrow$ when it was much greater than or less than the target, respectively. The number of events varies between sessions due to truncations ${ }^{\dagger}$ The number of events is small here because the experimental apparatus malfunctioned in the second half of these sessions. ${ }^{\ddagger}$ Patient 4 did not respond when testing his contralesional hand because it is plegic. . . . 124 


\section{List of Figures}

1.1 Brodmann's cytoarchitectural atlas adapted from (Garey, 1994). Sagittal views of Brodmann's areas on the lateral (a) and medial (b) surfaces of the

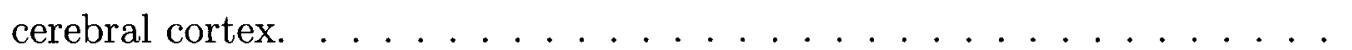

1.2 Illustration of the gross human neuroanatomy adapted from Kandel et al. (2000). The major regions are labelled in bold type, and the lobes of the cerebrum are labelled in plain type. . . . . . . . . . . . .

1.3 Illustration of neurons found in a cortical column of the human neocortex, adapted from Barr (1974). a, Various types of neurons shown to scale with their axon labelled (layer 3 pyramidal cell shown has diameter 10-50 $\mu \mathrm{m}) . \mathbf{b}$, Neuronal arrangement in a cortical column, consisting of six layers identified by the occupying cell type (height of a column varies throughout cortex between $1.5-4.5 \mathrm{~mm}$ )

1.4 Solution to equation (1.1) plotting the propagated action potential $-V(t)$, given the transmembrane conductance $g$ and its $\mathrm{K}^{+}$and $\mathrm{Na}^{+}$components, adapted from Hodgkin \& Huxley (1952) . . . . . . . . . . . . . .

1.5 Illustration of the Kolmogorov-Lorentz artificial neural network. The arbitrary continuous $n$-dimensional function $f$ is computed by the superposition of two continuous one-dimensional functions $\psi$ and $g$ as required in theorem

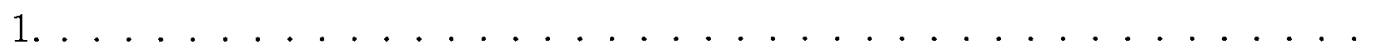


2.1 Coronal view of the somatotopic mapping of the human primary motor cortex adapted from Penfield \& Rasmussen (1950). . . . . . . . . . . . . 22

2.2 The sensorimotor circuits involving the basal ganglia and thalamus. Transverse (a) and coronal (b) sections adapted from Grey (1977). c, Illustration of the main interconnections between cortex, basal ganglia, and thalamus; adapted from Kandel et al. (2000). . . . . . . . . . . . . . . . 25

2.3 Illustration of the neurovascular unit in the cerebral cortex, adapted from Hamel (2006) . . . . . . . . . . . . . . . . . . . . 26

2.4 Inferior view of the brain revealing the large cerebral arteries forming the circle of Willis, adapted from Grey (1977) . . . . . . . . . . . .

3.1 Photographs of the hand grip portion of the experimental apparatus. a, The hand grip device in relation to a Canadian dollar. $\mathbf{b}$, The pressure sensors for both devices colour-coded (red for right hand; purple for left

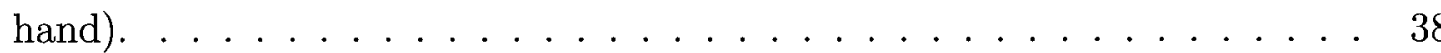

3.2 Plot of the BOLD signal measuring functional hyperemia after one-second stimuli are presented once, twice, and thrice with one-second interstimulus rest periods, adapted from Rosen et al. (1998). a, The raw signals show that the hemodynamic response compounds with additional stimuli. $\mathrm{b}$, Subtracting individual responses from the compound signal shows that subsequent responses are different from the original response. . . . . . . . . 39

3.3 Display frames seen by the subject during rest (a), when an event begins (b), and when the subject performs the motor response (c). . . . . . . 40

3.4 Photograph of the electronic portion of the experimental apparatus (top view). The signal inputs, the signal conditioning circuit, the data acquisition board, and the computer output are labelled. . . . . . . . . . . . . . 41

3.5 Diagram of the experimental apparatus interacting with the scanner and the subject ('A/D' represents the data acquisition board) $\ldots \ldots \ldots \ldots$ 
4.1 The autocovariance function estimate $\hat{\gamma}[\tau]$ plotted along the read-encode $x$-axis (a), the phase-encode $y$-axis (b), the slice-select $z$-axis $(\mathbf{c})$, and the time axis $(\mathbf{d})$. The phantom data were acquired in the $\mathrm{CP}(\mathrm{PN}$ on) RF coil

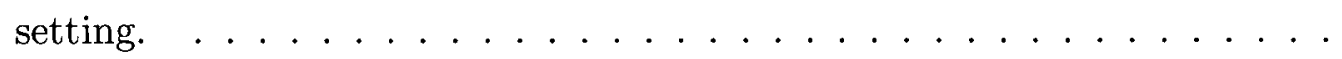

4.2 Comparison of the six possible MRI RF coil settings. a, The SNR is shown in $\mathrm{dB}$. $\mathbf{b}$, Plot of the six $\hat{\eta}[t]$ signals shown as percent change from the mean; the heavy black curve corresponds to $\mathrm{CP}$ (PN on). c, The adjacent intervoxel correlation coefficients along the read-encode $x$, phase-encode $y$,

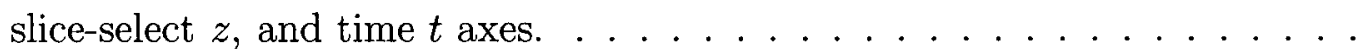

4.3 Simulated fMRI data. a, Modelled BOLD responses from normal subjects (signal 1) and CVD patients (signals 2,3). b, Event-related paradigm with interstimulus period randomly varying between 16-20 s (grey area indicates simulated BOLD response duration) $\ldots \ldots \ldots \ldots \ldots$

4.4 Typical autocovariance function estimates $\hat{\gamma}_{t}[\tau]$ computed from single voxel time series of length $T=150$ shown from head phantom (a), null fMRI data (b), and $b=9$ block resampling simulations from null fMRI data (c). The empirical correlation coefficient $\hat{r}[1]$ is shown along with the peak width shaded in grey.

5.1 Relation between data and their partition entropy. Obtaining a probability distribution of $H$ requires knowledge of the composition of functions $\mathcal{J}_{\min } \circ$

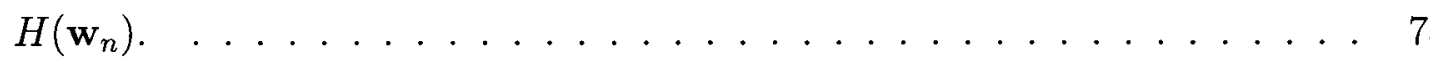

5.2 Comparison of partition entropy estimates between a null fMRI session and an event-related fMRI session.

5.3 Illustration of a $k$-partition obtained from a normal subject performing the event-related visual feedback controlled motor paradigm. Member voxels (green) appear in the cluster with maximum membership. The brain surface is rendered (grey) for intercluster comparison. . . . . . . . . . . 
6.1 Contiguity for a collection of two-dimensional clusters. In each case, the total number of member voxels (black) is $\left|X_{i}\right|=14$, and the smallest group size is set to $m=3 . \ldots \ldots \ldots \ldots \ldots \ldots \ldots$

6.2 Illustration of the effect of $r_{c}$ threshold values on the contiguity function for a typical voxel cluster with colour bar indicating $r\left(x, v_{i}\right)$ on a transverse anatomical overlay shown in radiological convention. Voxels forming a contiguous cluster $X_{i}$ are shown according to the colourmap scale that quantifies $r\left(x, v_{i}\right)$ for contiguity thresholds $r_{c}$ of 0.6 (a, blue square), 0.7 (b, green square), and $0.8(\mathbf{c}$, red square $) . \ldots \ldots \ldots . \ldots . \ldots 91$

6.3 Plot of a cluster centroid BOLD response signal (black), the corresponding motor response signal (red), and the event-related stimuli (grey) acquired throughout a neuroimage session from a normal subject. . . . . . . . . . 93

6.4 Illustration of the space-time characteristics of the clusters from a normal subject performing the event-related visual feedback controlled motor paradigm. The membership maps (bottom four rows) are displayed in transcranial, sagittal, and coronal projections seen from the superior, right, and posterior views, respectively. The voxels identified by each cluster have been circled in their corresponding colour on the GLM- $t$ map (top row) testing for the normal hemodynamic response at $p<0.001$. The contiguity threshold value is marked by a symbol on the contiguity functions. . . .

7.1 Overview of the fMRI analysis stages. The acquired neuroimage data are partitioned by an exploratory pattern recognition algorithm into a set of $k$ clusters. The set of clusters is then parameterised by $k$ features. The features are then used in a Bayesian model to determine the salience of each cluster. 
7.2 Histograms demonstrating the hierarchical and Gaussian form of cluster voxel correlations. a, Guassian-shaped distribution of $r(x, m)$ correlations for ten null fMRI sessions shows how the global signal can be biased in either direction. $\mathbf{b}$, frequency-normalised distribution (colour bar indicates relative frequency) of member voxel correlations (plotted along the ordinate) around their cluster (index along the abscissa) centroid correlation $r\left(v_{i}, m\right)$ within one fMRI session. These histograms demonstrate how motor response correlations are hierarchically distributed and global and cluster levels. . . . . . . . . . . . . . . . . . . . . . . . . 102

7.3 Bayesian hierarchical model parameters (adapted from Gelman et al. (2004)) used in the final stage of the analysis. The model parameters $(\alpha, \beta)$ are fit to the data $y$, such that each cluster feature $y_{i}$ is drawn from an underlying signal $\beta_{i}$. In turn, all underlying signals $\beta$ are drawn from a global signal $\alpha . \ldots \ldots \ldots$. . . . . . . . . . . . . . . . . . . 104

7.4 Simulation results from the proposed method. a, Box plots of paradigmcluster correlation values from the entire data set $\alpha$ and each cluster $\beta_{i}$ $(i=1, \ldots, k)$. The ${ }^{*}$ symbol denotes clusters that are significantly different from $\alpha$ with $p<0.05$. b, Significant voxel-cluster correlation maps for clusters 3-5 (membership threshold indicated by white bar on scale). c, Averaged voxel time series (events indicated by vertical grey bars). . . . . 113

7.5 Simulation results from the GLM- $t$ method. a, Significant voxel-paradigm $t$-maps obtained from the correct paradigm. $\mathbf{b}$, Significant voxel-paradigm $t$-maps obtained from the paradigm advanced by 4 seconds ( $t$-threshold indicated by white bar on scale). c, Averaged voxel time series (events indicated by vertical grey bars). . . . . . . . . . . . . . 113 
8.1 Summary showing the structural MRI (radiological convention) and motor responses from the stroke patients in comparison to a normal subject. The motor response signals from both hands are superimposed over each event occurring between the vertical grey lines shown. These data for each subject were acquired during two neuroimage sessions testing the right hand (top graph) and left hand (bottom graph).

8.2 Demonstration of the neuroimage quality in a normal subject (a), and in the presence of a large stroke infarction (b) and extracerebral paramagnetic implant (c). A considerable distortion in the anatomy of the coregistered and functional images in row $\mathrm{c}$ is appreciable. . . . . . . . . .

8.3 Graph of the distribution of the MCMC simulated Bayesian hierarchical model parameters for every cluster identified in the normal (black circles) and stroke (red crosses) groups. Each point in the graph represents the difference of means between the cluster and global signal correlations (plotted along the abscissa) and the cluster's SD of correlations (plotted along the ordinate). The boldfaced points were selected as representing a BOLD signal response to the event-related paradigm. . . . . . . . . . . . . . 128

8.4 Characterisation of the space-time structure of the BOLD signal identified in the normal (black) and stroke (red) groups. a, Plot of the mean contiguity function and its standard error for the selected clusters representing a BOLD response signal. $\mathbf{b}$, Graph of the causal cross-correlation maxima $\left(r_{m}, d\right)$ for the selected clusters representing a BOLD response signal. In order to distinguish the delay values (discretised as $T_{R}$ multiples), they were jittered using random noise uniformly distributed over $[-1,1]$ seconds. 
8.5 BOLD response signal from the normal group performing the event-related motor task with either hand shown in radiological convention on a coregistered transverse structural image from a normal subject. Neuroimage data from right-handed sessions are reflected across the midline. Voxels in the selected cluster maps from all 14 sessions are superimposed, and the colour scale indicates the number of sessions in which the voxels are significant. . 133

8.6 BOLD response signal from the stroke group performing the event-related motor task with the ipsilesional hand. The overlay is shown in radiological convention on a coregistered transverse structural image from a normal subject. Neuroimage data from left-handed sessions are reflected across the midline. Voxels in the selected cluster maps from all 7 sessions are superimposed, and the colour scale indicates the number of sessions in which the voxels were significant. Arrows indicate slice and hemisphere where the SMC response is expected according to normal group results. . . . . . . .

8.7 BOLD response signal from the stroke group performing the event-related motor task with the contralesional hand. The overlay is shown in radiological convention on a coregistered transverse structural image from a normal subject. Neuroimage data from right-handed sessions are reflected across the midline. Voxels in the selected cluster maps from all 7 sessions are superimposed, and the colour scale indicates the number of sessions in which the voxels were significant. Arrows indicate slice and hemisphere where the SMC response is expected according to normal group results. . . . . . 136 
8.8 Summary of neuroimage analysis results from normal subject 5 using her right hand. The Bayesian hierarchical model shows which clusters are selected by ${ }^{*}$ having Bayes' error $p<0.05$ relative to the global signal. The cluster voxels are shown (in green) from three computer rendered views, and the anatomical overlay, in radiological convention, corresponds to the slice containing the voxel with maximum centroid correlation. Cross-correlation and contiguity plots for all clusters are shown with the same scale. . . . 139

8.9 Summary of neuroimage analysis results from stroke patient 1 using her right hand. The Bayesian hierarchical model shows which clusters are selected by ${ }^{*}$ having Bayes' error $p<0.05$ relative to the global signal. The cluster voxels are shown (in green) from three computer rendered views, and the anatomical overlay, in radiological convention, corresponds to the slice containing the voxel with maximum centroid correlation. Cross-correlation and contiguity plots for all clusters are shown with the same scale. . . . 140 


\title{
List of Acronyms
}

\author{
ANOVA analysis of variance \\ AR autoregressive \\ BA Brodmann's area \\ BOLD blood oxygen level dependent \\ CBF cerebral blood flow \\ $\mathrm{CBV}$ cerebral blood volume \\ CMRglu cerebral metabolic rate of glucose \\ $\mathrm{CMRO}_{2}$ cerebral metabolic rate of oxygen \\ CNS central nervous system \\ CP circular polarisation \\ CWS compact well-separated \\ deoxy-Hb deoxygenated hemoglobin \\ DFT discrete Fourier transform \\ DTI diffusion tensor imaging \\ EEG electroencephalography \\ EPI echo-planar imaging \\ i.i.d. independent and identically distributed \\ FLAIR fluid attenuated inversion recovery \\ fMRI functional magnetic resonance imaging \\ GLM general linear model \\ LTI linear time-invariant \\ MCA middle cerebral artery \\ MCMC Markov chain Monte Carlo \\ MEG magnetoencephalography \\ MRI magnetic resonance imaging \\ OHREB Ottawa Hosptial Research Ethics Board \\ oxy- $\mathrm{Hb}$ oxygenated hemoglobin \\ PET positron emission tomography \\ PLS partial least squares \\ PN prescan normalisation
}


PNS peripheral nervous system

PSD power spectrum density

QED quod erat demonstrandum

$\mathrm{RF}$ radio frequency

SD standard deviation

SMC sensorimotor cortex

SNR signal-to-noise ratio

SPECT single photon emission computed tomography

SPM statistical parametric mapping

WSS wide-sense stationary 


\section{Chapter 1}

\section{Human Neurophysiology at the \\ System Level}

\section{$1.1 \quad$ Introduction}

Mankind's fascination with the brain reaches back amongst the earliest records of civilisation, first describing the human nervous system in ancient Egyptian hieroglyphs nearly four millennia ago (Breasted, 1930). Two of the major turning points in our early understanding of the nervous system are mentioned now because they form the core of modern neuroscience and emphasise the central theme of this thesis - that the advancement of analytical methods leads to new scientific insights. A detailed historical account of these events is given by Finger (1994).

The first turning point, dating back to ancient Greece circa $5^{\text {th }}$ century B.C., was the idea that the mind and intellect resides solely within the brain and not in the heart as was previously believed. It was first proposed in the scholarly work of Alcmaeon, resulting from his dissection of the human brain, and was established a century later during the study of seizures, head injuries, and their treatments recorded in the Hippocratic corpus, a series of medical treatises written by Hippocrates amongst other anonymous 
ancient Greek physicians. The second turning point, taking place in late $19^{\text {th }}$ century Europe, was the establishment of the neuron doctrine. It began with the improvement of microscopy, which allowed the investigators to resolve brain tissue at the cellular level. A cellular theory was consequently proposed by German physiologist Theodor Schwann in 1839 , which proposed that organisms were entirely composed of discrete biological cells. However, the nerve cells, having a topology unique from those of other organs, form a complex network, or reticulum, with fine processes branching between the cell bodies. Thus, despite Schwann's cellular theory, none could verify whether the nervous reticulum was actually composed of individual nerve cells whose fine processes were not fused but merely touched. The compelling evidence in favour of the cell theory came from a new tissue staining method by Italian physician Camillo Golgi in 1873, which allowed the staining of individual cells thereby revealing microscopically the termination of their processes. The scientific work proving the cellular nature of the nervous system came from Spanish histologist Santiago Ramón y Cajal in 1890. The final link explaining the communication mechanism between processes of neurons was discovered, and named "synapse," by English neurophysiologist Sir Charles Scott Sherrington in 1897. The neuron doctrine remains today at the foundation of modern neuroscience, upon which we consider that our behaviour, thoughts, and actions are ultimately manifest from the trafficking of information signals spanning the reticulum of the one million million $\left(10^{12}\right)$ neurons that make up the human brain (Kandel et al., 2000). These two major turning points of neuroscience came from advancements in analytical methods, namely in neurosurgery, microscopy, histology, and biochemistry.

Since the neuron doctrine was established, much neurophysiological research has continued at the single neuron level and now also at the system level to further understand how the brain orchestrates its activity to yield a coherent and intelligent human organism. This chapter will first review general neuroanatomy and physiology, and the steps taken toward developing a theory of computational neuroscience. Secondly, it will elaborate on 
some of the modern empirical and theoretical methods developed to study brain function. It will also point out the methods still lacking and, in this regard, state the focus of this thesis. The variety of topics covered in this chapter may seem broad, yet they ought to be understood in the context that neuroscience has become a consilience of specialised disciplines. Therefore, study in one discipline must diligently consider the implications from results found in the other disciplines so that the theory of neuroscience be an integrative one, capable of mutually corroborating the phenomena observed.

\subsection{General neuroanatomy and physiology}

Neurophysiology, the study of brain function, is an anatomically based subject because the brain possesses a highly consistent and organised topography whose distinguishable landmarks serve to identify its features as well as the presence of any abnormalities. Such anatomical consistency led Talairach \& Tournoux (1988) to first plot a stereotaxic brain atlas derived from a single post-mortem female subject. A vastly improved and generalised version derived from over 7000 human samples created through an International Consortium of Brain Mapping known as the ICBM atlas is the recognised standard today (Mazziotta et al., 2001). These maps are of crucial importance to neuroimaging studies as they provide the anatomical basis for the acquired structural and functional image data. The anatomical structure is also present at the microscopic level, where the arrangement and diversity of the neurons found throughout the cerebral cortex vary depending on the region examined. The consistency of this microscopic structure led to the cytoarchitectonic classification of the cortical grey matter, the brain tissue containing the neuronal cell bodies suspended in a fibrous matrix called neuropil. The most widely used cytoarchitechtonic atlases are those of Brodmann (Garey, 1994) and, to a lesser extent, those of von Economo \& Koskinas (2008). Brodmann's atlas is shown in figure 1.1.

Decades of experience primarily involving clinical cases of patients with brain injury 

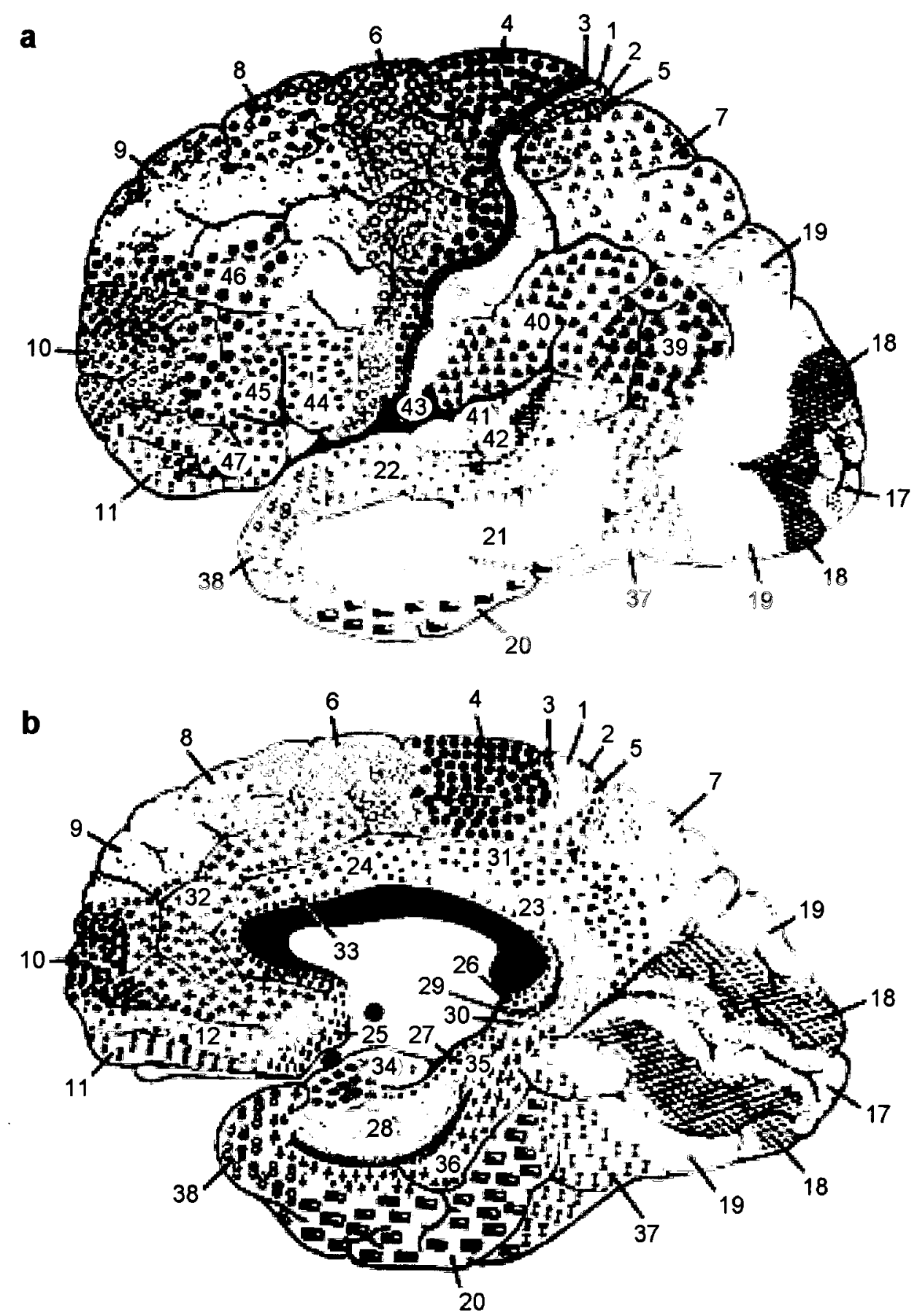

Figure 1.1: Brodmann's cytoarchitectural atlas adapted from (Garey, 1994). Sagittal views of Brodmann's areas on the lateral (a) and medial (b) surfaces of the cerebral cortex. 


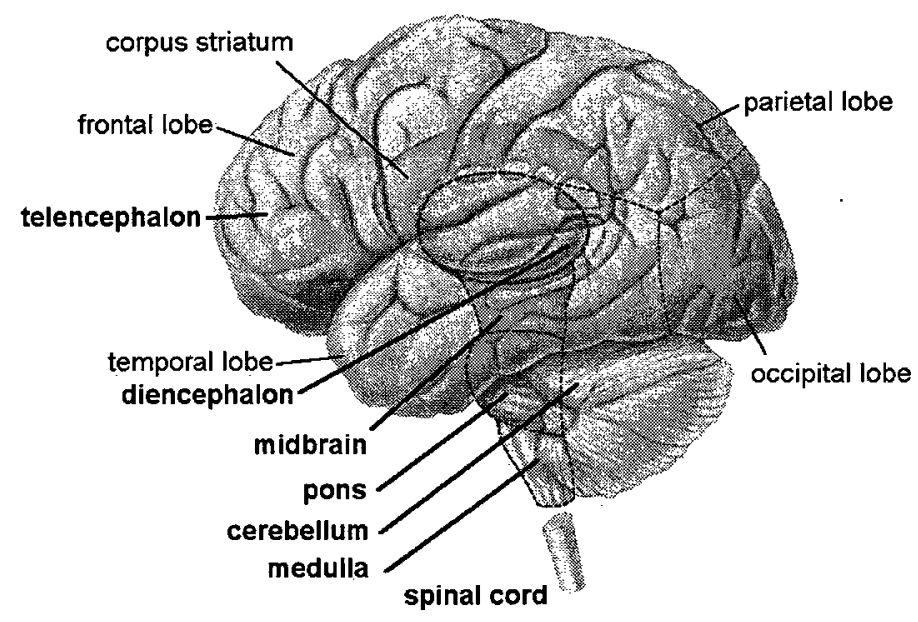

Figure 1.2: Illustration of the gross human neuroanatomy adapted from Kandel et al. (2000). The major regions are labelled in bold type, and the lobes of the cerebrum are labelled in plain type.

and neurological disease as well as non-human primate studies led to the understanding that the divorso furchions of ine brain are indecd locatised throughout the cortex. The major regions of the central nervous system (CNS) are now briefly described based on the work of Barr (1974) to clarify the interconnection of these localised functional areas. The subcortical tissue, i.e., the white matter, is sparse of neuronal cell bodies and instead contains the long axonal processes that are predominantly ensheathed by myelin. The white matter forms the inter-cortical network throughout the brain and communicates to the peripheral nervous system (PNS) throughout the body via the spinal cord. Figure 1.2 illustrates the major regions of the CNS:

- The spinal cord communicates paired sensory and motor nerves throughout the body. A transverse section of the cord reveals a central ' $\mathrm{H}$ ' shaped profile of grey matter surrounded by peripheral white matter that projects the nerve fibres longitudinally to the body.

- These spinal fibre tracts continue through the medulla, which also contains neuron cell clusters that send fibres to the cerebellum and others to the pons.

- The pons consists of two parts: the dorsal part is akin to the medulla, and the 
basal part provides extensive connectivity between each cerebral hemisphere and its contralateral cerebellar hemisphere. These connections are very important for efficient execution of motor activity.

- The midbrain is much like the brain stem regions described above containing sensorimotor pathways and, in addition, the auditory and oculomotor cranial nerves.

- The cerebellum receives data from most of the sensory systems and the cerebral cortex. It works to influence motor neurons supplying skeletal musculature by regulating muscle toneus in relation to gait. While it is primarily responsible for such subconscious movements, it is also known to be involved in the execution of specialised movement based on individual experience.

- The diencephalon is the central core of the cerebrum anatomically and functionally, whose largest component is the thalamus, a dense gray matter region that receives data from all sensory systems (except olfaction) and projects them to the sensory areas of the cerebral cortex. It also reverberates circuits between cortical areas concerned with higher functions, e.g., voluntary movements and cognition. Other nuclei of the thalamus are in circuit with the limbic system, and motor pathways linking cerebellum to corpus striatum and cerebral cortex. It also synthesises hormones and releases them in the blood stream via the pituitary gland. Finally, the retina and hence the visual system, is an immediate derivative of the diencephalon.

- The telencephalon consists of the corpus striatum and both cerebral hemispheres. The corpus striatum wraps around the thalamus and its nuclei project fibres primarily in three directions: i) intra-hemispherically, ii) inter-hemispherically, and iii) those converging towards the thalamus. It has roles in the limbic system and all sensory systems (again, except olfaction). Each cerebral hemisphere consists of a highly convoluted cortex whose topography is typically described by the convex folds called the gyri and the concave grooves called sulci. The hemispheres appear to be virtually 
symmetric at a glance but, in fact, have some typical asymmetries. It is within the cerebral cortex that the neural activity for the highest functions take place.

The cerebral cortex is special in humans because its evolution away from the ancient paleocortex is unmatched throughout the animal kingdom. In lower vertebrates, the paleocortex remains the dominant cortical matter, while in mammals, most of it has been replaced by neocortex. The human cerebral cortex consists of $90 \%$ neocortex. To quote Canadian physician and anatomist Murray Barr on the remarkable distinction of our species in nature (Barr, 1974):

"The unique place of the human species is an endowment conferred by an expanse of neocortex that is possessed by no other animal."

\subsection{Toward a theory of computational neuroscience}

With the knowledge of function localisation and the interconnected nature of the CNS, one can consider the neuron doctrine as providing the building blocks for information processing within a highly distributed computational network. The microscopic complexity and quantity of neurons, combined with their numerous interconnections and inherent inaccessibility, make the comprehensive monitoring of the resulting network's operation impossible to achieve with the current technology. However, this does not preclude the possibility of extensive study of the brain post-mortem, albeit, no longer functioning. Nor does it preclude observing in vivo small subsections in detail, or even the entire system at a macroscopic scale. These problems of data acquisition serve to provide empirical evidence to obtain a theoretical understanding of how information is processed in the brain. There are only limited experimental data on the fundamentals of neural computation taking place at a system level, and consequently, much of the theoretical research comes from the study of individual neurons, small populations of in vitro cultured neurons, and mathematical models (Feng, 2004). Two seminal quantitative results, one at the neuron level and the 
a

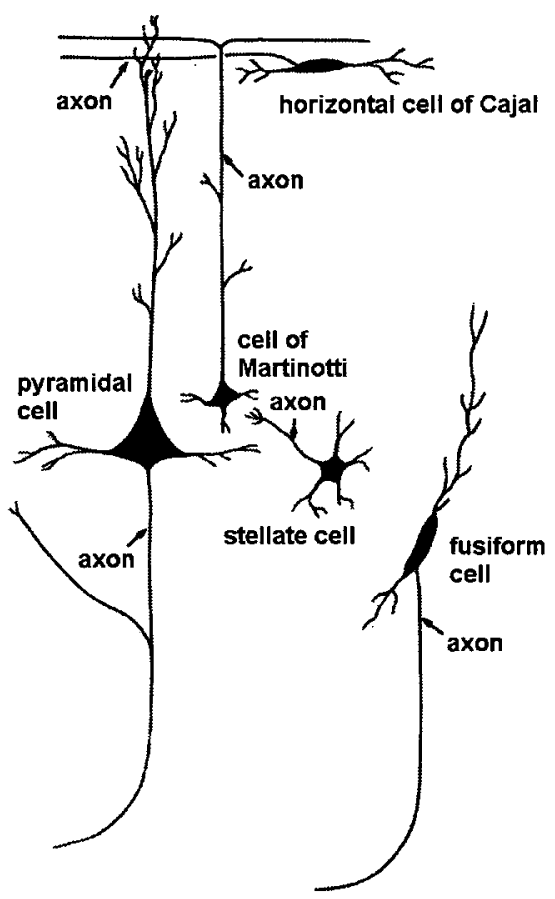

b

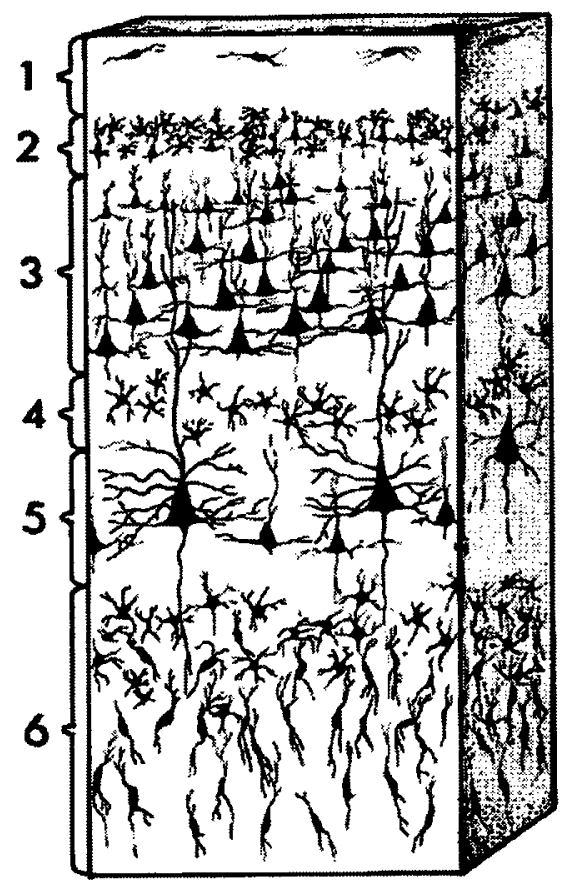

Figure 1.3: Illustration of neurons found in a cortical column of the human neocortex, adapted from Barr (1974). a, Various types of neurons shown to scale with their axon labelled (layer 3 pyramidal cell shown has diameter $10-50 \mu \mathrm{m}$ ). b, Neuronal arrangement in a cortical column, consisting of six layers identified by the occupying cell type (height of a column varies throughout cortex between $1.5-4.5 \mathrm{~mm}$ ).

other at the network level, are described here because their impact has shaped much of the current paradigm on brain function. To relate these results to the neuroanatomy, figure 1.3 illustrates the geometry of neurons and how they are organised into stratified columns that form the neocortex.

The first came from the physiological study by Hodgkin \& Huxley (1952) of the electric current through the surface membrane of the axon. Their study led to a quantitative model of the initiation and propagation of localised ionic currents that travel along the axon away from the cell body, an all-or-nothing phenomenon called the action potential. These currents work to depolarise the resting-state transmembrane ionic potential when its ionic permeability changes, hence allowing the equilibration of the transmembrane concentration gradients of $\mathrm{K}^{+}$and $\mathrm{Na}^{+}$primarily, as well as other "leakage" currents. The dynamics and velocity of conduction of the action potential travelling across the mem- 


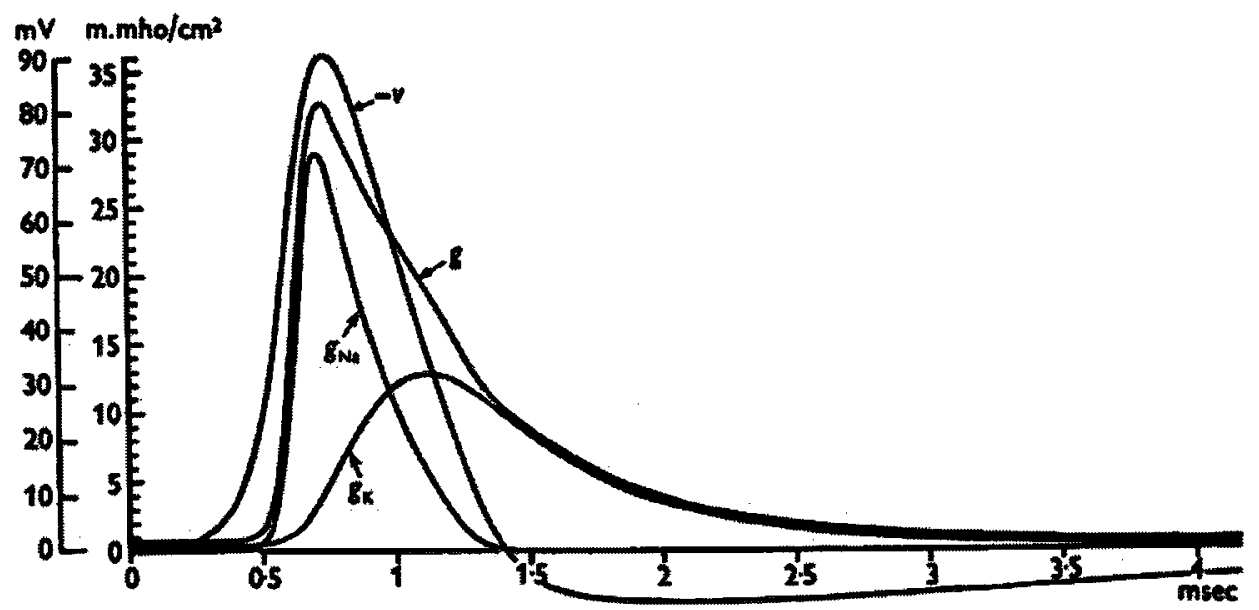

Figure 1.4: Solution to equation (1.1) plotting the propagated action potential $-V(t)$, given the transmembrane conductance $g$ and its $\mathrm{K}^{+}$and $\mathrm{Na}^{+}$components, adapted from Hodgkin \& Huxley (1952).

brane were derived from this model and expressed by the voltage function $V=V(x, t)$ satisfying the partial differential equation (Hodgkin \& Huxley, 1952)

$$
\frac{a}{2 R_{2}} \frac{\partial^{2} V}{\partial x^{2}}=C_{M} \frac{\partial V}{\partial t}+\bar{g}_{K} n^{4}\left(V-V_{K}\right)+\bar{g}_{N a} m^{3} h\left(V-V_{N a}\right)+\bar{g}_{l}\left(V-V_{l}\right)
$$

The left hand side represents the current membrane density, and the four terms on the right hand side represent the membrane's capacitance, and the ionic conductance of the $\mathrm{K}^{+}, \mathrm{Na}^{+}$, and leakage channels, respectively. Figure 1.4 from Hodgkin \& Huxley (1952) plots versus time the propagated action potential $-V$ and the transmembrane conductance that satisfies equation (1.1) and agrees with their experimental data. This work revealed a key mechanism of neuronal communication: sequences of all-or-nothing pulses, which in turn trigger the diffusion of biochemical neurotransmitters across the synapse to reach the adjoining neuron.

The second result addresses the question of how a network of communicating neurons could collectively form a system with meaningful output, instead of gibberish. The problem actually was set in a purely mathematical context, on the algebraic reconstruction of polynomial functions, posed by an influential mathematician of the $19^{\text {th }}$ and $20^{\text {th }}$ 
centuries David Hilbert. The question essentially was whether one could express any continuous multivariate function by means of the sum and composition of a finite number of continuous univariate functions. This question was surprisingly answered in the affirmative by Kolmogorov (1957), showing that any continuous $n$-dimensional function $f$ can be represented by exactly $(n+1)(2 n+1)$ total copies of two continuous one-dimensional functions. Formally, the result is known as the Kolmogorov superposition theorem and can be stated as follows.

Theorem 1 (Lorentz (1976)). Let $f:[0,1]^{n} \rightarrow \mathbb{R}$ be a continuous function. Then $f$ can be rewritten as follows. Let $\epsilon>0$ be a real number, then

$$
f=\sum_{k=1}^{2 n+1} g\left(z_{k}\right)+k,
$$

where $g:[0,1] \rightarrow \mathbb{R}$ is a suitable continuous function (depending on $f$ and $\epsilon$ ), and for all $k$,

$$
z_{k}=\sum_{j=1}^{n} \lambda_{k} \psi\left(x_{j}+\epsilon k\right) .
$$

Here $\lambda_{k} \in \mathbb{R}$, and $\psi$ is a monotonically increasing Lipschitz real function on $[0,1]$ that does not depend on $f$ nor $\epsilon$.

This theorem describes the artificial neural network shown in figure 1.5, called the "Kolmogorov-Lorentz" network, where the first layer has $n(2 n+1)$ nodes, each made from the one-dimensional function $\psi$, and the second layer has $2 n+1$ nodes, each made from the one-dimensional function $g$. This relatively simple feed-forward network is all that is necessary to replicate any continuous $n$-dimensional function desired. Consequently, this result is recognised as of importance in computational neuroscience (Kürková, 1991), since it shows in theory the power of a distributed computational network: a machine capable of responding precisely to multivariate inputs by means of very simple computations taking place in parallel. Much subsequent research has been put into answering the subsequent question of how to choose the suitable univariate function $g$. Neural networks under the umbrella of statistical machine learning has become a fundamental topic of computational neuroscience and pattern recognition in general (Devroye et al., 1996). A statistical theory 


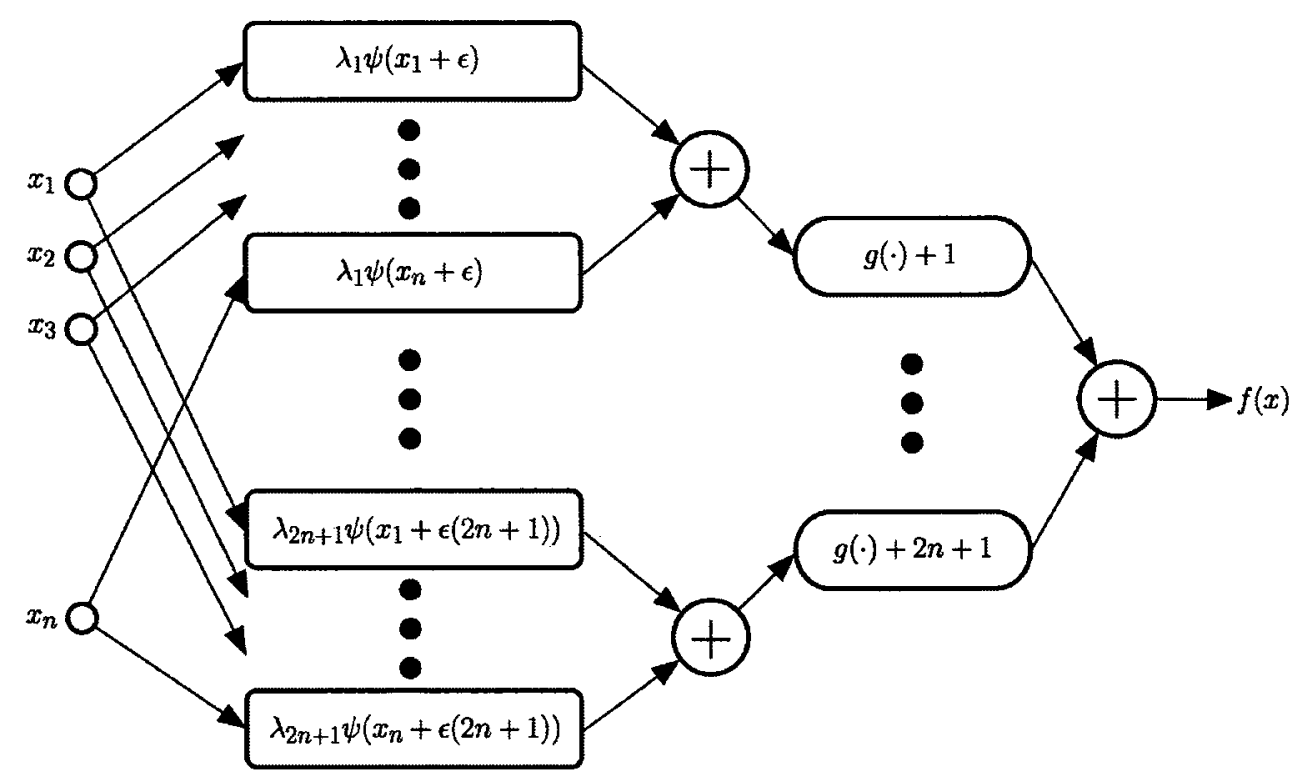

Figure 1.5: Illustration of the Kolmogorov-Lorentz artificial neural network. The arbitrary continuous $n$-dimensional function $f$ is computed by the superposition of two continuous one-dimensional functions $\psi$ and $g$ as required in theorem 1 .

of learning created by Vladimir Vapnik and Alexey Chervonenkis (Vapnik, 1998) now serves to quantify "how much" a machine like a neural network among others can learn from a given data set.

It is clear that to begin applying these ideas to the brain at large will require much effort. The goal is to obtain a solid understanding of the native architecture and basic instructions required for the execution of operations on data circulating within the nervous system, and upon which routines are built for data management and information coding, and furthermore, upon those even more sophisticated programs for adaptation and learning, etc. Despite the remarkable progress in computational neuroscience over the past century, and the energetic debates on various coding theories, a comprehensive theory of this architecture and its implementation throughout the CNS remains largely a mystery (Feng, 2004). Nonetheless, the excitement from the results and discoveries in this relatively new field have brought us to the brink where the neuron doctrine can indeed be considered a realistic and theoretically plausible basis to understand how the brain works. 


\subsection{Gathering empirical evidence of brain function}

The evidence gathered from experimental data can be classified into two complementary groups. Histological, biochemical, and genetic studies approach the problem from the bottom-up, examining the elementary building blocks of cells and their matrix. Psychological, surgical, and medical imaging studies follow a top-down strategy, examining the system behaviour in normal and abnormal conditions. Corroboration of the findings from these disciplines is essential for a sound understanding of neurophysiology and for the validation of the theories they are based upon. Neuroimaging methods are useful in this respect and are briefly introduced to demonstrate this point.

Results from histological studies of the tissue density, organisation, and intragyral connectivity of neurons are being compared with those obtained from human structural neuroimage data acquired by magnetic resonance imaging (MRI) that estimate cortical thickness and curvature from $\mathrm{T}_{1}$-weighted scans (Lyttelton et al., 2009), and are beginning to resolve the gross cytoarchitecture at sub-millimetre resolution (Thomas et al., 2008). Post-mortem white matter fibre tractography studies are now being compared with diffusion tensor imaging (DTI), an MRI technique to reconstruct the main directions of water diffusion within each voxel (Tuch et al., 2003). DTI can estimate the average direction of axonal fibres in voxels of white matter by regularising the diffusion data between voxels to follow three-dimensional curves (Savadjiev et al., 2006).

The physiological processes taking place at the system level, e.g., the regional electromagnetic activity from the superposition of neural action potentials, and its associated regional metabolic activity and regulation of blood flow, are primarily studied with functional neuroimaging methods. This is the case because human physiological studies require an application of minimally invasive methods in order to generalise the observations to their respective populations. 


\subsubsection{Direct cortical recordings}

The regional electromagnetic fields generated by neuron action potentials are best recorded by electrodes temporarily implanted directly into the cortex during surgery. This most invasive procedure seldom takes place, since there are fewer clinical centres with the expertise to conduct these studies, and fewer consenting patients who clinically need the suitable type of neurosurgery. However, much can be learned from these recordings due to their high quality. Hochberg et al. (2006) demonstrated the control of a prosthetic device, a computer mouse cursor, using neuronal ensemble recordings from a $4 \mathrm{~mm}^{2}$ electrode array implanted in the cortex of a tetraplegic patient. Quiroga et al. (2005) identified individual neurons specifically responsible for the recognition of people or objects from similar electrode array recordings in an epileptic patient. Of course, planning these specific experiments, namely, deciding where to put the electrodes, depends mostly on functional neuroimaging studies that measure activity throughout the entire brain during such tasks of motor control or recognition.

\subsubsection{Electromagnetic field recordings}

Non-invasive measurements of electromagnetic activity are typically done with electroencephalography (EEG), i.e., recordings from a net of electrodes covering the scalp. The EEG signal quality is relatively poor due to the electrical insulation properties of the skull and scalp. Moreover, source localisation from EEG data is a severely ill-posed inverse problem. Consequently, spatial resolution is limited to the localisation of the average electric dipole on the superficial part of the cerebral cortex adjacent to the skull. The temporal resolution, however, is excellent being on the order of one millisecond. Therefore, EEG can provide useful information about the causality of the responses to brief stimuli from general cortical centres.

An alternative method is magnetoencephalography (MEG), also non-invasive, which rather measures the magnetic field induced by regional neural activity using very 
sensitive superconductive sensors that similarly cover the skull. The spatial resolution is generally better than EEG because the neural activity-induced magnetic fields are not so much distorted by the scalp and skull. However, MEG also suffers from the severely ill-posed inverse problem and is also most sensitive to the superficial surface of the cerebral cortex. The temporal resolution of MEG is comparable to EEG.

\subsubsection{Metabolic and vascular imaging}

Neural activity requires energy, which is generated by the metabolism of oxygen and glucose supplied by the cerebrovasculature. At the arteriolar level, the brain vessels are endowed with a mechanism to regulate the cerebral blood flow (CBF) so that cortical tissue receives a steady blood supply independent of systemic fluctuations in arterial pressure (Kuschinsky, 2000). Secondly, a task-related increase in regional neural activity is followed by a concomitant regional increase in $\mathrm{CBF}$; a mechanism called functional hyperemia or the hemodynamic response (Iadecola \& Nedergaard, 2007). Nuclear imaging methods such as positron emission tomography (PET) and single photon emission computed tomography (SPECT) are capable of measuring the cerebral metabolic rates of oxygen $\left(\mathrm{CMRO}_{2}\right)$ and glucose (CMRglu), as well as CBF by monitoring the quantity of circulating water, oxygen, or glucose molecules labelled with suitable radioactive atomic isotopes such as ${ }^{15} \mathrm{O},{ }^{18} \mathrm{~F}$, or ${ }^{99 \mathrm{~m}} \mathrm{Tc}$. Thus, these methods provide an indirect measure of neural activity that is dependent of this relationship with metabolic and vascular function. The radiolabelled tracers are considered minimally invasive and are typically introduced into the system by intravenous injection. Nuclear imaging studies involving normal subjects are acceptible; however, they require proper justification because ionising radiation levels involved are significant. PET is generally more sensitive than SPECT, having superior spatial resolution and contrast (Knešaurek \& Machac, 2006). PET has a spatial resolution of about $5 \mathrm{~mm}$, whose images are typically reconstructed by applying the filtered back projection algorithm on data representing the trajectory end-points of emitted 
gamma particles. Thus, unlike EEG and MEG, PET imaging is not a severely ill-posed problem and has a relatively uniform sensitivity so that the deep brain regions can also be observed. Because PET reconstructs images from an accumulation of emission events over time, its temporal resolution is not independent of image quality and is relatively poor. Dynamic PET imaging can have a temporal resolution on the order of tens of seconds.

\subsubsection{Blood oxygenation imaging}

Nuclear imaging studies investigating the relationship between neural activity, metabolism, and blood flow have shown that functional hyperemia entails a disproportionate increase of blood flow and metabolism. Fox \& Raichle (1986) first showed that $\triangle \mathrm{CBF}>\triangle \mathrm{CMRO}_{2}$ during neural activation induced by somatosensory stimulation, which is interpreted as meaning that a large increase in $\mathrm{CBF}$ is needed to make small increases in oxygen metabolism possible (Villringer, 2000). Subsequently, Fox et al. (1988) and Madsen et al. (1995) showed that $\triangle \mathrm{CMRglu}>\triangle \mathrm{CMRO}_{2}$ during neural activation induced by visual and cognitive tasks, and the measured lactate production only accounted for a small proportion of the glucose uptake. These findings suggest that oxidative glycolysis is the main energy source of neural activity, and that the fate of the remaining uptaken glucose is still open to debate (Villringer, 2000).

The combination of these physiological phenomena and the physical fact that the hemoglobin molecule is diamagnetic when oxygenated $(\mathrm{oxy}-\mathrm{Hb})$ and paramagnetic when deoxygenated (deoxy-Hb) led to the discovery of a blood oxygenation level dependent (BOLD) contrast for MRI (Ogawa et al, 1990), and its use in the functional magnetic resonance imaging (fMRI) of the human brain (Kwong et al., 1992). The BOLD contrast serves to indirectly measure changes in neural activity based on changes in the regional concentrations [oxy-Hb] and [deoxy-Hb]. During functional hyperemia, an increase in [oxy- $\mathrm{Hb}]$ takes place since $\Delta \mathrm{CBF}>\Delta \mathrm{CMRO}_{2}$. Therefore, the local MRI signal intensity increases within the vicinity of neural activity because fewer paramagnetic deoxy- $\mathrm{Hb}$ molecules are 
present to destructively alter the magnetic susceptibility within the microvasculature.

To infer neural activity from these relative changes in the BOLD signal, however, relies upon assumptions of the hemodynamics described above, whose metabolic and vascular components are not directly measured by the BOLD signal. However, Ito et al. (2005) verified this expected BOLD response in normal subjects by comparing acquired BOLD fMRI data with subsequent measurements of $\mathrm{CBF}, \mathrm{CMRO}_{2}$, and the oxygen extraction fraction using ${ }^{15} \mathrm{O}$ PET during rest and during a hand motor task. Such validation studies indeed show that fMRI is a powerful technique for observing the cerebrovascular response of neural activity in vivo, having several important advantages over other functional neuroimaging methods. Foremost, it is non-invasive and requires no administration of contrast agent or intervention of any kind. Thus, it poses no additional risk beyond that of a standard MRI scan, making it suitable for the extensive study of consenting normal subjects and patients in their natural state. It has the highest spatial resolution of the functional neuroimaging modalities. For example, a standard scanner operating at 1.5 Tesla magnetic flux density has a resolution on the order of $2 \mathrm{~mm}$ and can image the entire brain in about 2 seconds in a repetitive manner. Image reconstruction is not ill-posed and is done by inverse Fourier transform of the magnitude and phase components of the MR radio frequency data. Finally, fMRI has roughly uniform sensitivity throughout the field of view and can be accompanied by submillimetre resolution structural $T_{1}$ or $T_{2}$ scans for precise anatomical localisation throughout the brain.

\subsection{Analytical methods for neuroimage data}

This chapter began with a discussion of how our understanding of neurophysiology grew in consequence of the advancements in the analytical methods used to study the brain. As the methods began to probe the subtleties of the subject, the investigators increasingly became aware of its sophistication, leading to new questions and eventually new ideas. The 
material surveyed in this chapter hopefully provided the reader with a basic understanding of human neurophysiology at the system level as well as various ways it can be studied. Only with such background can one undertake the problem of analysing neuroimage data.

The success of a neuroimaging experiment depends on the consideration of this understanding when designing the experiment and analysis methodology as well as during the interpretation of results. First, one must avoid associating the acquired images with the absolute truth and rather interpret them as a representation of the subject under study, prone to error and uninformative of certain properties of the subject (Joyce, 2008). Secondly, finding ourselves in the 'information age' has provided us with vast quantities of data. Especially in the case of neuroimaging, as our technological progress moves forward, the complexity of the data begin to approach that of the working human brain. Indeed, these data sets have become large, multidimensional, and sensitive to many factors beyond what is tolerable for the analysis by visual inspection. Consequently, we now move toward the 'analysis age', where our dependence on powerful analytical methods grows substantially. An example of this transition is clear in the work by Quiroga et al. (2005). The first in vivo single neuron action potential recordings were done in 1963, and cortical recording studies began to explore language and processing function in 1988. However, only in 2005 could modern analysis techniques provide the robust identification of single neuron action potentials from vast amounts of data, making them amenable to interpretation by human experts (Abbott, 2009).

The three main stages of neuroimage analysis methods are introduced with brief examples of their implementation. In the following sections, visualise that functional neuroimage data as a set of time series signals localised throughout regions of the brain that were recorded in one session during which the subject was exposed to stimuli of some kind. 


\subsubsection{Pattern recognition}

Pattern recognition methods have been applied to time series data as an exploratory step for the identification of regions carrying similar signals or those carrying signals that potentially respond to a stimulus. This is a reasonable objective, since the brain is a heterogeneous system with some degree of functional substructure. For example, Mourão-Miranda et al. (2005) trained the support vector machine algorithm to discriminate between BOLD fMRI data acquired during resting and active states, and subsequently, applied the machine to predict a new subject's state for each given image. Esposito et al. (2005) applied a supervised clustering algorithm to characterise BOLD fMRI data from multiple subjects by identifying similar independent components present across all subjects. Rajapakse \& Zhou (2007) applied a dynamic Bayesian network algorithm that analysed the temporal connectivity between distinct brain regions using BOLD fMRI data.

\subsubsection{Parametric modelling}

Parametric models of the data sets are also useful in characterising the diversity found between subjects and groups. The most widely used model is the general linear model (GLM) as proposed by Friston et al. (1995) and Worsley \& Friston (1995) that forms a statistical parametric map (SPM) of the voxels from functional neuroimage data. The GLM essentially performs a linear regression of the data onto explanatory variables that represent the stimuli and the expected hemodynamic response. McIntosh et al. (1996) proposed the application of partial least squares (PLS) to neuroimage data. PLS maximises the covariance matrix of the data set by grouping the voxels optimally within blocks that are defined by explanatory variables similar to those in the GLM (McIntosh \& Lobaugh, 2004). Recently, de Pasquale et al. (2008) proposed a Bayesian approach to first account for temporal autocorrelation in BOLD fMRI signals with an autoregressive model, and second, to represent the model parameters within a joint probability density function. 


\subsubsection{Statistical inference}

Methods of performing inference on the model's actual state based on the observed data have also been approached in various, complementary ways. Both SPM and PLS are readily amenable to classical hypothesis testing based on $t$ and $F$ statistics within an analysis of variance (ANOVA) design. An alternative approach is the use of computer-intensive resampling tests, i.e., bootstrap or randomisation techniques described by Efron \& Tibshirani (1991), which have been applied to both GLM and PLS (McIntosh \& Lobaugh, 2004; Wang et al., 2007). A third approach that is emerging is Bayesian inference. All Bayesian models (e.g., Rajapakse \& Zhou (2007); de Pasquale et al. (2008)) are readily amenable to Bayesian inference by applying Bayes' rule to their joint probability density model. Bayesian models have also been implemented within the GLM framework (Penny et al., 2007).

\subsection{Focus and summary of the thesis}

This thesis focuses primarily on the development of novel analysis methods for functional neuroimage data with the objective to further develop our understanding of brain function at the system level. In particular, the experimental studies undertaken were aimed toward the characterisation of the sensorimotor system using BOLD fMRI data acquired from healthy volunteers and ischemic stroke patients.

The motivation for studying stroke is the prevalence of the disease and the ensuing chronic disability that burdens its surviving victims. In North America, stroke is the leading cause of chronic cognitive and motor impairment; moreover, it is the third leading cause of death (Murphy \& Corbett, 2009). Consequently, rehabilitation therapy attempting to minimise stroke's burden-of-disease rely on diagnostic methods to monitor patient recovery. Therefore, neuroimaging methods play an important role in stroke rehabilitation research by monitoring the recovery progress. In addition, functional neuroimaging might 
provide enough information to accurately predict a patient's outcome under a particular therapy. This predictive power would be most useful for the case-by-case selection of the optimal rehabilitation therapy. The next chapter will review the impact stroke has on the sensorimotor system, the conclusions drawn from previous studies, and the questions posed in this research.

The motivation for developing novel analysis methods stem from the known complexity of the CNS and consequently of the acquired functional neuroimage data. Chapters 3 and 4 are dedicated to the acquisition of BOLD fMRI data. Chapter 3 justifies the experimental design and the MRI parameters chosen. It also describes the subject manipulations performed with special consideration for stroke patients. Chapter 4 begins by reporting the results from the preliminary analysis of the data to describe the BOLD signal and noise characteristics; it concludes with the data simulation method used for quantifying algorithm performance. Chapters 5-7 are the core of the thesis that develop the functional neuroimaging analysis methods for i) the objective recognition of substructure in the data set, ii) the characterisation of the BOLD signal space-time structures identified, and iii) a Bayesian hierarchical model for evaluating the statistical significance of these structures. The purpose of this material is to provide a flexible, exploratory analysis method that can characterise the space-time structure of functional neuroimage data. Chapter 8 applies the proposed analysis to characterise the space-time structure of BOLD fMRI data from recovering ischemic stroke patients with motor deficits. This chapter will compare the characteristics from normal and stroke data to determine their ability to discriminate between these populations and furthermore to describe the relationship between these data and the degree of motor impairment.

The material from chapters 4-8 has been subsequently submitted for publication in peer-reviewed international academic journals dedicated to the advancement of data analysis and medical imaging methodology. 


\section{Chapter 2}

\section{The Sensorimotor System}

\subsection{Introduction}

The preceding chapter reviewed the general neurophysiology and its neural, metabolic, and vascular substrates. It also introduced the neuroimaging methods to study brain function at a system level. This chapter now details the subsystem investigated in the remainder of the thesis--the sensorimotor system-beginning with its functional anatomy. For a correct interpretation of the neuroimage data acquired, which relies on functional hyperemia, it is also necessary to review the known cellular mechanisms that control the cerebral microcirculation. Then, the impact that stroke has on this subsystem is described along with the implications for neuroimaging studies. Finally, a discussion of the presented evidence is given with an emphasis on the consequent questions addressed in this research.

\subsection{Functional anatomy of the sensorimotor system}

The sensorimotor system consists of the subcircuits dedicated to somatosensory and motor function. Although distinct, these subcircuits may be considered as a system because of their natural interaction in both body sensation and movement. 


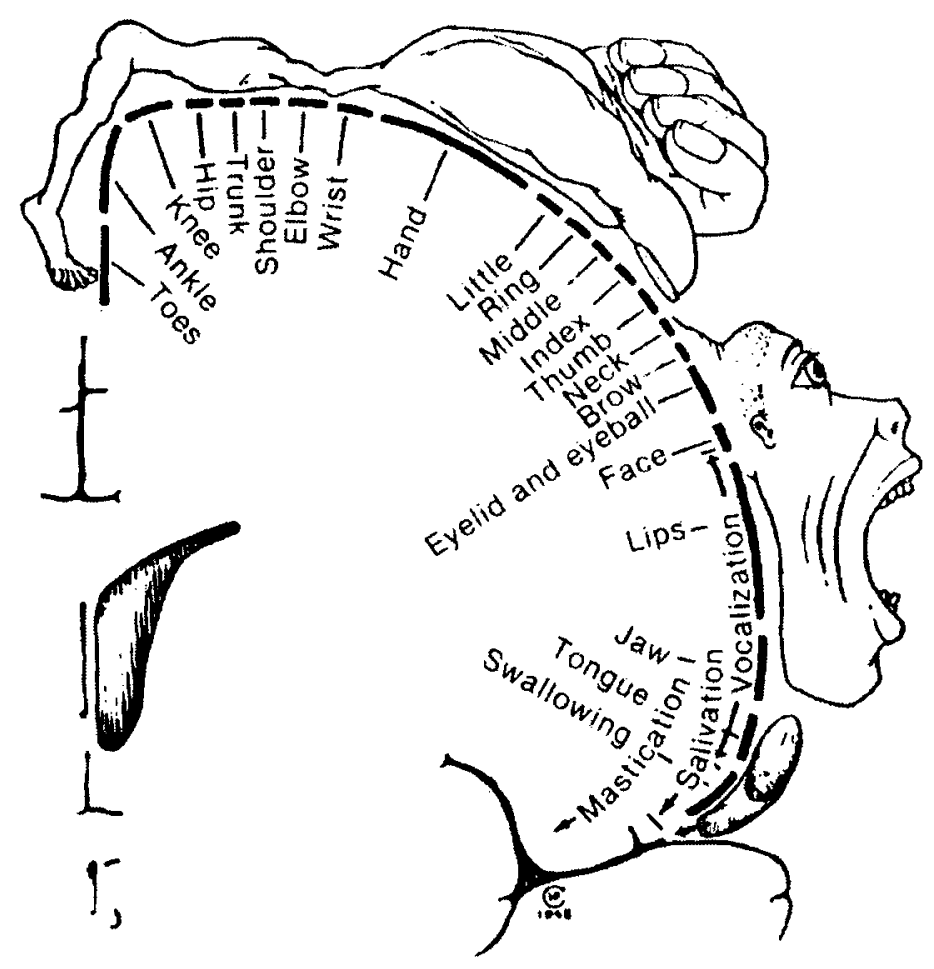

Figure 2.1: Coronal view of the somatotopic mapping of the human primary motor cortex adapted from Penfield \& Rasmussen (1950).

\subsubsection{The sensorimotor cortex}

The planning and execution of voluntary movements take place in the primary motor cortex, also called M1, forming Brodmann's area (BA) 4 (see figure 1.1, page 4). M1 coincides with the precentral gyrus on the lateral and medial surfaces of the frontal lobes. The giant pyramidal neurons, or Betz cells, in M1 send their axon toward the peripheral nervous system (PNS) via cortico-striate fibres to effectuate skeletal muscle control. The distribution of these neurons in M1 form a topographic representation of the body, i.e., a somatotopic mapping, known as the homunculus shown in figure 2.1. To complement M1, the primary somatosensory cortex receives sensory input from the PNS via thalamo-cortical fibres and is located immediately posterior to M1 forming BA 3, 1, and 2, coinciding with the postcentral gyrus on the lateral and medial surfaces of the parietal lobes. The somatotopic mapping also forms a homunculus, not identical to that in M1. These cortical areas are 
collectively referred as the sensorimotor cortex (SMC). The SMC has been extensively studied in humans, and insightful reviews are given by Kandel et al. (2000) and Reis et al. (2008).

Anterior to the SMC are the supplementary motor and premotor areas (BA 6) that are also closely involved in motor control. The precise functions of these areas, however, remain controversial. The supplementary motor area lies on the posterior end of the superior frontal gyrus that coincides with the dorsomedial part of BA 6. It is thought to link cognition with action because it receives cortico-cortical inputs anteriorly from frontal cortex and propagates cortico-cortical outputs posteriorly to M1, which are somatotopically organised. Evidence suggests that this area "primes" motor programs before they are executed (e.g., preparing the movements needed for reaching out to grab an object). This postulate is based on studies demonstrating its activation in self-initiated movements as opposed to movements performed when commanded. A detailed review of the supplementary motor area is given by Nachev et al. (2008). The premotor area lies directly inferior to the supplementary motor area, coinciding with the dorsolateral part of BA 6 . Its principal inputs come from the somatosensory area in the posterior parietal cortex (BA $5 \& 7$ ) and sends outputs alongside those of M1 but in fewer number toward the motor circuits of the PNS. Along with experimental evidence relating premotor area activity and limb motion, it is thought to primarily control the orientation and coordination of movements (Kandel et al., 2000). The posterior parietal cortex then appears to complement the secondary motor areas both in function and location, being posterior to the SMC on the dorsomedial surface of the parietal lobe. It is likely involved in the integration of sensory information as part of a feedback circuit for targeted movements that rely on vision and spatial perspective.

The connectivity of the motor areas on the frontal cortex suggests an anterior-toposterior hierarchical organisation of function. Starting from M1 and proceeding anteriorly along the dorsomedial line, the substructures encountered within the supplementary mo- 
tor area (see Nachev et al. (2008) for details) and beyond appear to be associated with functions of increasing level of abstraction but that ultimately depend on basic motor function. Indeed, Badre et al. (2009) recently provided evidence supporting this hierarchy by demonstrating a direct correlation between the position of focal frontal lobe lesions in patients and their performance deficits during motor tasks involving gradually increasing levels of abstraction. Perhaps motor programs are built in an integrative manner as abstract information from cognitive centres in the prefrontal cortex proceeds posteriorly toward M1.

\subsubsection{The basal ganglia and cerebellum}

The term basal ganglia refers to substructures of the corpus striatum that are involved in motor function, in particular the putamen and the globus pallidus. Together, these centres receive input from the cortex and send output back to the cerebral cortex via thalamo-cortical circuits as well as toward the PNS. The main substructures and their connectivity are illustrated in figure 2.2. The accumulated experimental evidence implicates the basal ganglia in the control of movement extent and speed. Turner et al. (2003), for example, have demonstrated the modulation of regional CBF in the putamen during the performance of whole arm motor tasks involving the pursuit of on-screen targets by joystick manipulation.

The cerebellum has a remarkably distinct cytoarchitecture from the rest of the CNS, possessing an organisation of neuron types not found elsewhere in the brain. It consists of two hemispheres that communicate contralaterally to the cerebral hemispheres; e.g., the left cerebral hemisphere is primarily connected to the right cerebellar hemisphere. As described in chapter 1 , the cerebellum plays an important role in muscle tone regulation for posture and in the co-ordination of voluntary movements (Barr, 1974; Kandel et al., 2000). 

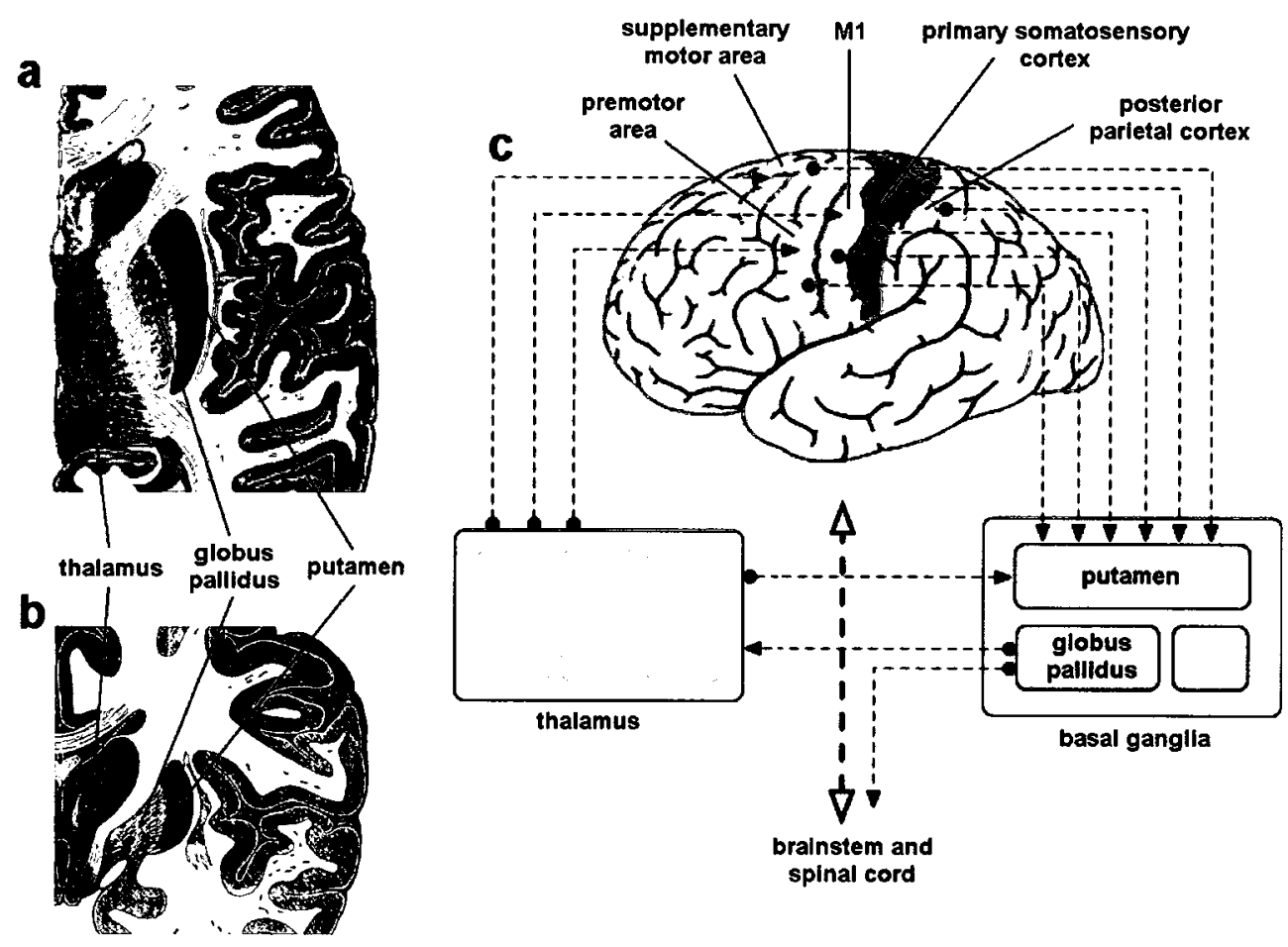

Figure 2.2: The sensorimotor circuits involving the basal ganglia and thalamus. Transverse (a) and coronal (b) sections adapted from Grey (1977). $\mathbb{c}$, Illustration of the main interconnections between cortex, basal ganglia, and thalamus; adapted from Kandel et al. (2000).

\subsection{The neurovascular unit}

Understanding the cellular mechanisms that regulate the cerebral circulation is a major goal of neuroscience. Hamel (2006) and Iadecola \& Nedergaard (2007) provide dense reviews of the current body of knowledge on the subject discussed here.

The brain depends on a continuous blood supply to provide the oxygen and glucose necessary for neuronal activity. Any interruption in this supply causes the deprived neurons to suddenly stop working and die. Consequently, the cerebrovasculature is endowed with cellular mechanisms that provide a steady blood flow in the presence of daily fluctuations of arterial pressure, called cerebrovascular autoregulation, and those that provide a regional increase in $\mathrm{CBF}$ that matches the regional increases in neuronal activity, i.e., functional hyperemia. A good understanding of these mechanisms will help in the interpretation of metabolic and vascular neuroimage data and may also lead to therapeutic 


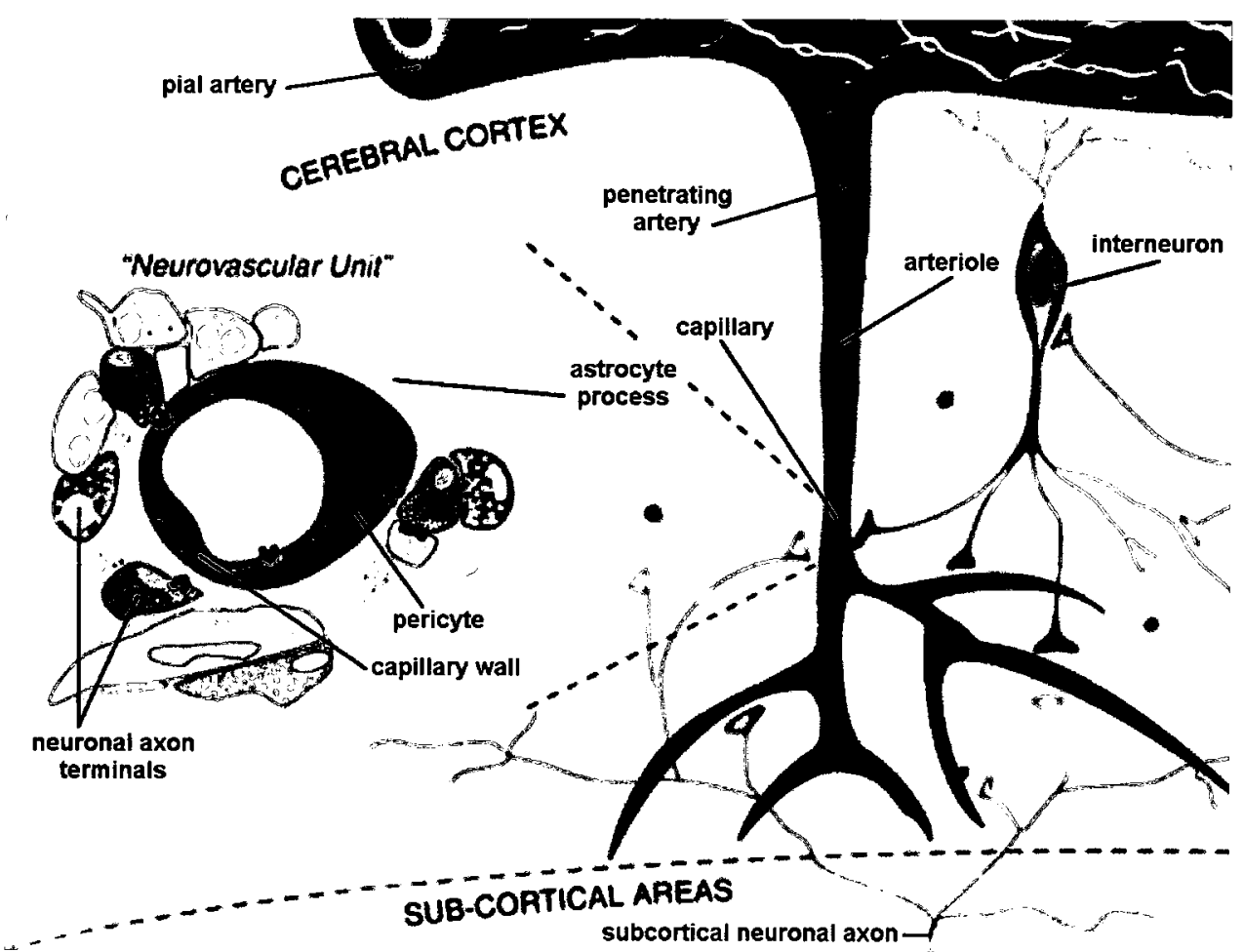

Figure 2.3: Illustration of the neurovascular unit in the cerebral cortex, adapted from Hamel (2006).

avenues for cerebrovascular disease.

The current body of evidence indicates that CBF is locally controlled within the brain's grey matter by a tripartite neurovascular unit composed of neurons, astrocytes, and vascular pericytes that constrict or dilate blood vessel diameter at the arteriolar and capillary levels, collectively called the microvasculature. Figure 2.3 illustrates the structure of the neurovascular unit. Astrocytes belong to the second class of cells, called glia, that along with neurons make up the CNS. These cells are abundant throughout the grey matter and serve to transport chemicals essential for the metabolic processes of neurons. At the capillary and arteriolar level, the pericytes surround the vessel wall and provide the mechanical forces for vasoconstriction. These pericytes are in turn surrounded by astrocytic processes, also called endfeet, and to a lesser extent by the terminals from neuronal axons.

The spatial organisation of the astrocytes suggests that they are the dominant 
mediators of the microcirculation because their cell bodies are spread out to occupy nonoverlapping spatial domains throughout the grey matter, and their endfeet envelop virtually the entire microvascular surface. Moreover, each astrocyte communicates with its neighbouring astrocytes and with all neuronal synapses in their domain via adjoining processes. Therefore, these cells are well positioned to control the local vascular supply by releasing vasoactive agents directly on the pericytes in response to neuronal or glial activity.

Interneurons, i.e., small neurons with their axon terminating locally (e.g., stellate cell in figure 1.3a, page 8) and axon terminals arriving from subcortical fibres are also known to interact with the pericytes directly and via glial endfeet (Hamel, 2006). Kocharyan et al. (2008) have shown in the in vivo rat that the activation of perivascular neuronal terminals, projected from nearby interneurons or from distal neurons via subcortical fibres, strongly influence microvascular tone. In this view, interneurons behave as integrators of the neuronal activity level in their region and consequently have the means to adjust microvascular perfusion accordingly.

There is also recent evidence suggesting that astrocytes may also adjust the microvascular tone independent of neuronal activity. Gordon et al. (2009) have shown in the rat brain that astrocytes can control the microvasculature with various signals, some being independent of neuronal activity, and that they can either constrict or dilate vessels depending on the oxygen availability. Moreover, some of these control signals are not related to neuronal activity. An example of this is the spontaneous intrinsic activity from astrocytes observed in ex vivo tissue slices (Iadecola \& Nedergaard, 2007). In light of this, the astrocyte's role can no longer be thought as merely a passive mediator for focal neuronal activity. On the contrary, it is an active agent capable of controlling vascular tone in response to a number of signals some that may be independent of neuronal activity. Although these mechanisms are in place, the findings do not necessarily reflect the reality in vivo, since the experiments required that tissue be removed from its natural 
environment. Until these effects can be observed in vivo, their functional significance in the normal brain remains inconclusive.

Although the neurovascular unit influences microvessel tone, these arteriolar and capillary vessels are only responsible for one-third of the vascular resistance in the brain, while the remaining two-thirds are determined by the pial arteries travelling on the surface of the brain (Iadecola \& Nedergaard, 2007), also shown in figure 2.3. Consequently, the neurovascular unit can only redistribute $\mathrm{CBF}$ to a limited extent. While this may be sufficient for cerebrovascular autoregulation, it is not for functional hyperemia (Hamel, 2006; Girouard \& Iadecola, 2006; Iadecola \& Nedergaard, 2007). For example, during SMC activation, the vasodilation propagates upstream and relaxes the smooth muscle cells around the appropriate superficial pial artery. The mechanism that allows this retrograde vasodilation remains unclear but is thought to involve the release of multiple vasoactive agents from neurons and glia that target the vasculature at different levels to produce a timely increase in CBF matching the spatial extent of the neuronal activity (Hamel, 2006; Girouard \& Iadecola, 2006).

The consensus is that functional hyperemia involves the interaction of various cells and signalling mechanisms that act in concert to produce the hemodynamic response. These results have serious implications on the interpretation of vascular neuroimage data, such as BOLD or CBF contrasts, since the data may be representative of an aggregate demand of which only a fraction is born of neuronal activity. This concern applies manifold in disease due to the abnormal cellular behaviour related to dysfunction or repair. Indeed, although the BOLD signal is empirically known to be a reliable indicator of neuronal activity in normal subjects, it has been shown to fail in cases of cerebrovascular disease (Rossini et al., 2003). Therefore, only when the neurovascular unit is fully understood and its state can be verified in vivo, can a quantitative model of neuronal activity via neurovascular coupling be justified. 


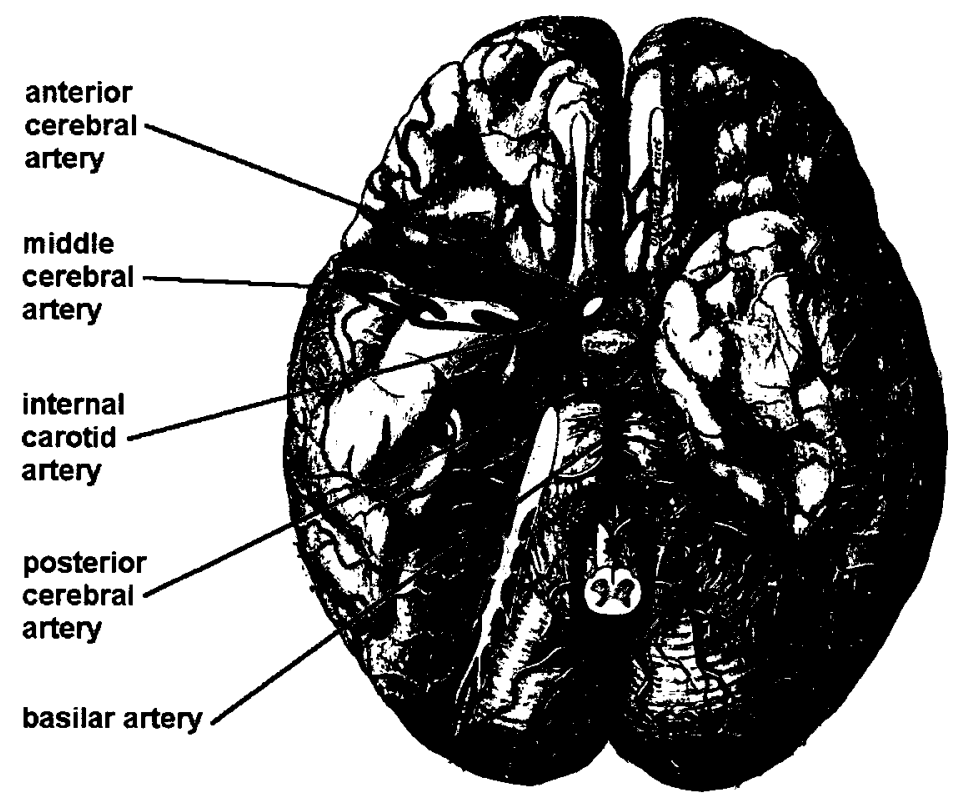

Figure 2.4: Inferior view of the brain revealing the large cerebral arteries forming the circle of Willis, adapted from Grey (1977).

\subsection{The effects of ischemic stroke}

Ischemic stroke is the result of an interruption of sufficient duration and extent in the blood supply to the brain (Murphy \& Corbett, 2009). Focal ischemia typically occurs from the occlusion of a large cerebral artery branching from the circle of Willis, shown in figure 2.4. In the acute phase, the hypoperfused cerebral territory receives an inadequate supply of nutrients and also begins to accumulate toxic metabolic byproducts that lead to the infarction of the brain tissue (Doyle et al., 2008). Consequently, the functional role of the infarcted tissue is permanently lost. Thus, if the victim of stroke survives the initial insult, the chronic effects are often severely disabling and can result in persistent deficits of the sensory, motor, and cognitive faculties. Fortunately, brain tissue is perfused by multiple arteries, and so the extent of infarcted tissue is mediated by a redundant collateral supply, which results in a smaller infarct core surrounded by silent but viable tissue called the ischemic penumbra (Murphy et al., 2006). The pathophysiology that the neurons, glia, and vascular cells undergo following stroke depends on the degree of hypoperfusion experienced in the acute phase. For example, the ischemic core may become infarcted within minutes, 
while the penumbra may eventually die within days by other cellular processes such as apoptosis and inflammation (Doyle et al., 2008).

Alterations of the cerebral circulation take place after stroke. These patients are initially in a state of vasoparalysis and later exhibit impairment of cerebrovascular autoregulation (Girouard \& Iadecola, 2006). In the acute phase, Murphy et al. (2006) were able to accurately predict the fate of ischemic tissue by comparing the CBF and cerebral blood volume (CBV) levels between hemispheres of unilaterally occluded patients. They found that ischemic tissue that avoided infarction exhibited a prominent mismatch between $\mathrm{CBV}$ and $\mathrm{CBF}$ : an increased $\mathrm{CBV}$ and decreased $\mathrm{CBF}$ relative to what is seen in the non-occluded hemisphere. This is understood as a vasodilatory response to prevent the further decline of $\mathrm{CBF}$ during low perfusion pressure conditions. Altered autoregulation can also persist during the chronic phase of stroke. Mandell et al. (2008) were able to demonstrate impaired cerebrovascular reactivity in the affected hemisphere using an induced hypercapnia stimulus. Here cerebrovascular reactivity is defined in terms of the end-tidal partial pressure of $\mathrm{CO}_{2}\left(p_{\mathrm{ET}} \mathrm{CO}_{2}\right)$ as the ratio $\Delta \mathrm{CBF} / \Delta p_{\mathrm{ET}} \mathrm{CO}_{2}$, with an impairment being 2 standard deviations (SD) below the control group average.

Investigations measuring increases in neuronal activity and functional hyperemia were undertaken to determine whether the observed vascular alterations resulted from an uncoupling of the neurovascular unit or by changes in neuronal activity itself. In chronic stroke patients with mild neurological impairment, Bundo et al. (2002) studied interhemispheric differences in neuronal activity using MEG and the resting state CBF using PET during evoked by brief nerve stimulation events. The ischemic hemisphere had a reduced $\mathrm{CBF}$, and also their MEG data suggested a compensatory change in the neuronal activity had taken place, yet the neuronal response to the stimulus was present and remained timely in all patients. It is unfortunate, however, that they were not able to measure CBF during functional hyperemia because PET cannot temporally resolve CBF during a brief event stimulus. Rossini et al. (2003) performed a similar study, this time using BOLD to observe 
functional hyperemia and obtained striking results. They demonstrated that in chronic unilateral stroke patients, neuronal activity can take place without functional hyperemia in either the ischemic hemisphere or even both hemispheres. All ten patients exhibited the increased neuronal activity during nerve stimulation in both hemispheres; however, half of the patients did not undergo functional hyperemia in either the affected hemisphere or altogether. The authors surmised from global cerebrovascular reactivity data from clinical transcranial Doppler measurements that functional hyperemia did not take place in these patients because their microvasculature was already maximally dilated to preserve CBF as was observed by Murphy et al. (2006), discussed above. Nonetheless, the evidence here is compelling and demonstrates that the attenuation and even disappearance of functional hyperemia during neuronal activity can in fact occur after stroke.

Perhaps the most remarkable phenomenon after stroke is the patient's capacity to recover from the impairments incurred from infarction. Despite the permanent loss of neurons in the infarcted territory, the re-emergence of sensory, motor, and cognitive ability can occur from the reorganisation of existing neuronal networks that learn the required functional roles (Murphy \& Corbett, 2009). Capitalising on this recovery mechanism, called neuronal plasticity, is of great importance in reducing stroke's burden-of-disease. Indeed, a large part of today's efforts are aimed at understanding how and when plasticity takes place in the recovering brain, and how these reorganised networks translate into the recovery of the faculties needed for daily life (Murphy \& Corbett, 2009). A pair of studies by Ward et al. $(2003 a, b)$ were the first to thoroughly study functional hyperemia after stroke using BOLD fMRI in cross-sectional and longitudinal patient groups compared to age-matched normal subjects. Furthermore, these studies focused on the sensorimotor system and correlated the presence of the expected hemodynamic response with the degree of recovery using a battery of clinical outcome measures. Their results indicated that patients with poor recovery exhibit additional recruitment of SMC and motor-related areas beyond those seen in normal subjects. Notably, the cortical recruitment occurred 
in the supplementary motor and premotor areas as well as in the posterior parietal area. Additional areas involved the corpus striatum, thalamus, and cerebellum. Furthermore, throughout the six month post-stroke observational period in all patients, the areas gradually decreased as a function of recovery but independent of initial severity or recovery rate.

\subsection{Imaging stroke recovery with fMRI}

The literature review presented above supports the notion that functional neuroimaging is in a unique and tenable position to monitor the pathophysiology and recovery therefrom after ischemic stroke. Neuroimaging may perhaps prove indispensable for the validation of novel rehabilitation or drug therapies that aim to maximise neuronal plasticity by providing the investigators with spatial and temporally resolved indices of effectivity throughout the brain. Stroke can take on many pathological forms and degrees of severity (Doyle et al., 2008); its impact will also depend on the patient's age and medical history (Murphy \& Corbett, 2009). Hence, these factors must be considered on a case-wise basis to determine the optimal therapeutic strategy (Murphy \& Corbett, 2009). While quite speculative at

present, perhaps functional neuroimaging could play a central role in stroke recovery by determining the therapeutic strategy to yield the best possible outcome on a case-by-case basis. It is encouraging at least to see that the longitudinal data from Ward et al. (2003a) and Murphy et al. (2006) suggest that acute phase imaging may have some predictive power of final outcome.

The complexity of functional hyperemia foretells the challenges in interpreting the BOLD signal, especially in patients with cerebrovascular disease. The linear transform model relating neural activity to the hemodynamic response is a reasonable approximation in normal subjects and is attractive for its analytical simplicity (Boynton et al., 1996). However, it has been shown in later studies that, even between normal subjects, non- 
linear variations of the spatial extent and temporal profile of the hemodynamic response are appreciable (Rajapakse et al., 1998; de Pasquale et al., 2008). Furthermore, it has also been observed to vary within subjects depending on the stimulus duration and frequency of occurrence (Vazquez \& Noll, 1998). Finally, age-related changes have also been extensively observed in the normal population (Huettel et al., 2001; D'Esposito et al., 2003). In cerebrovascular disease, alterations of the hemodynamic response such as temporal delays or advances (Carusone et al., 2002; Roc et al., 2006), attenuation (Huettel et al., 2001; Pineiro et al., 2002), negative responses (Röther et al., 2002; Seghier et al., 2004), or even the absence of a response (Rossini et al., 2003) have been reported in the literature. These variations are presumably informative of the neurovascular state of the subject and if possible should be considered. Therefore, it is best to employ analytical methods that are flexible enough to identify and distinguish between such case-specific response patterns.

Studying stroke recovery presents additional challenges. The magnitude and location of the infarcted territory will vary between patients and will introduce specific stimulus-related effects on the BOLD signal. Hence, some studies only enrol "first-ever" stroke patients whose stimulus-specific functional anatomy is spared from infarction. In SMC-related studies, for example, ischemic damage in the subcortical sensorimotor circuits (e.g., corpus striatum and thalamus) can also complicate cortical activity due to the disruption of thalamo-cortical and cortio-striate communication. Another challenge of extensive damage is that the patients acquire atrophic distortions of the cerebral topography, which reduces the precision of anatomical localisation. Head movements can be more pronounced in patients, and so comfortable restraints may be necessary to maintain data quality. Finally, the experimental apparatus must be minimally invasive and cannot compromise the patient's clinical needs and, finally, the performance of any tasks must not be strenuous for the patient. These additional challenges must be met, rather than avoided by curbing the enrolment of impaired patients because their impairment arises, after all, from the very disease we face. 


\subsection{Discussion}

The material presented in chapters 1 and 2 provide the subject background necessary to approach the problem of interpreting functional neuroimage data in the context of cerebrovascular disease. Furthermore, the reviewed physiology of the sensorimotor system will be necessary for the validation of results obtain from the experiments undertaken in this research. In particular, working with the sensorimotor system is advantageous for several reasons. First, it can be stimulated by simple hand motor tasks that can be performed by normal subjects and stroke patients. Second, the motor responses can be simultaneously monitored for comparison with the neuroimage data. Third, it has been previously studied using various experimental paradigms and analysis methods; these studies will be useful in validating the results obtained in this research.

In normal subjects, functional hyperemia evoked from a particular stimulus is known to assume a relatively consistent spatial extent and temporal profile. However, there is enough evidence showing that these characteristics can change dramatically in the presence of cerebrovascular disease. Therefore, the nature of these alterations reflects the underlying pathophysiology. This thesis seeks to answer questions on whether the BOLD signal evoked from sensorimotor stimulation is informative of this process. Can we distinguish between normal subjects and stroke patients by looking at the space-time structure of their BOLD signals? If so, can we specifically characterise the structures relating to ischemic stroke and, hence, reasonably speculate on the underlying pathophysiology? Focusing on the stroke patients, can we relate their BOLD signal characteristics to the degree of motor impairment? Can we distinguish between different stages of stroke recovery from the signal characteristics observed in individual patients? If so, how do the characteristics evolve as recovery progresses? Additionally, neuronal plasticity commonly takes place after ischemic stroke, and distinct cerebral areas take on functional roles that were lost to infarction. Evidence suggests that the location of these recruited areas are not arbitrary but are rather functionally related to the destroyed tissue. Therefore, it is 
natural to ask how the role of recruited tissue might differ from role of the native one, and whether their hemodynamics are informative of this difference.

It is not tenable to approach these questions with standard analytical neuroimaging methods because they were not designed for pattern recognition but rather to test hypotheses concerning the presence of a particular response. Neither is the visual examination of the raw data set reasonable, due to the data's dimensionality and the subtle nature of the underlying BOLD signal. Instead, this thesis proposes a novel neuroanalytical method and aims to show that an exploratory pattern recognition method is capable of objectively identifying the space-time structural characteristics of the data, thereby making the isolated responses amenable to expert visual inspection and interpretation. 


\section{Chapter 3}

\section{Data Acquisition Methods: Part I Experimental Design and Neuroimaging}

\subsection{Introduction}

The introductory chapters reviewed the academic literature concerning the neurological systems involved and their evolution after stroke so that the choices motivating the data acquisition and analysis can be justified. The material in the next two chapters cover the data acquisition methodology used in this research, whose objectives are i) to systematically reproduce functional hyperemia in the sensorimotor system, and ii) to simultaneously observe the nervous system response and the cerebrovascular response.

This chapter first describes the experimental design that was developed in our laboratory to focus on observing functional hyperemia elicited by a novel visual feedback controlled hand motor task. Here, the implementation of the stimulus apparatus is given describing how the stimuli are presented and how the motor responses are recorded. Next, the neuroimage data acquisition method is detailed, including the pertinent $M R$ scanner specifications, the MR pulse sequence and protocols used for structural and functional imaging as well as the data preprocessing steps used to co-register the data sets and improve BOLD signal quality. Finally, the ethical and procedural considerations for human 
subjects are described, with an emphasis on the extra care needed for participating stroke patients. The chapter ends with a discussion of the benefits and drawbacks of the practical aspects of our method. The material presented in this chapter was primarily conceived by my co-supervisor Dr. M. J. Hogan and his collaborators. However, I did participate throughout my doctoral research in the implementation and refinement of the experimental apparatus and the stimulus paradigm used in our laboratory.

\section{$3.2 \quad$ Experimental design}

In these experiments, we decided to evoke functional hyperemia by requiring the subject to perform a hand motor task. The rationale follows from the above-mentioned research objectives and dealing with the environmental constraints imposed by the MR scanner. First, the hand representation on the SMC is disproportionately large (see figure 2.1, page 22), and consequently, a vascular response will likely be easier to observe with neuroimaging. Moreover, simple hand movements can be performed almost effortlessly in a consistent manner without requiring the movement of other body parts. This is especially relevant for systematically reproducing the BOLD signal, since the subject is required to lie supine and restrain from any head movements at all times. Second, hand movements are the natural choice for the subject to interact with a sensor to record their responses. Voluntary movements, such as gripping an object, occur within $500 \mathrm{~ms}$ of the cue to respond and so provide a timely correlate that can by simultaneously measured during neuroimaging. For this reason, we decided to have the subject hold a palm-sized ball in each hand that can be squeezed on given cues. Each ball is connected by tubing to a pressure sensor that monitors the pressure continuously throughout the experiment. Figure 3.1 shows the hand grip device and the pressure sensor mounting panel. Each hand grip device consists of a $100 \mathrm{ml}$ silicone evacuator bulb (Bard Inc., Covington, USA) attached to $15 \mathrm{~m}$ of Tygothane tubing with $6.35 \mathrm{~mm}$ outer and $4.76 \mathrm{~mm}$ inner wall diameters (Saint-Gobain, 


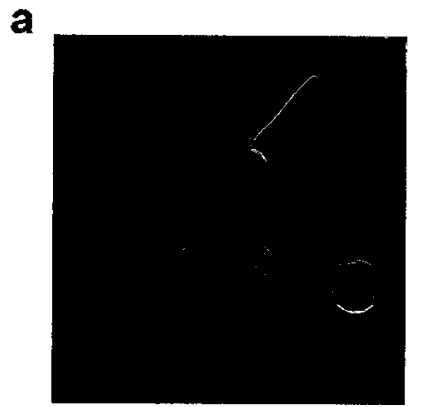

hand grip device

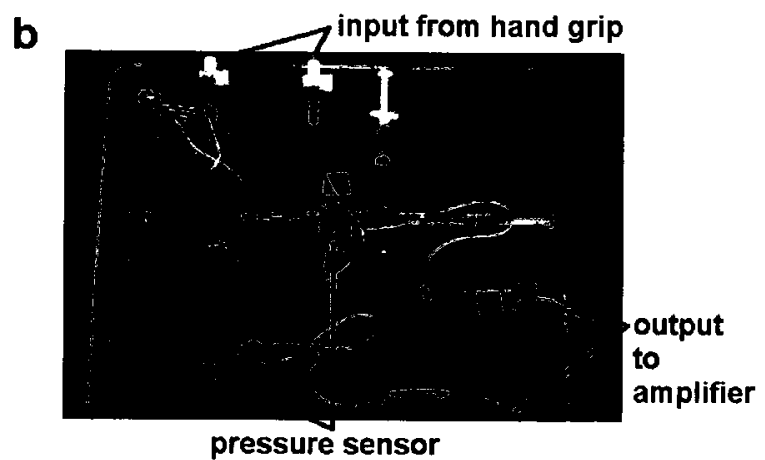

Figure 3.1: Photographs of the hand grip portion of the experimental apparatus. $\mathbf{a}$, The hand grip device in relation to a Canadian dollar. $\mathbf{b}$, The pressure sensors for both devices colour-coded (red for right hand; purple for left hand).

Akron, USA). Each sensor is a Deltran I disposable pressure transducer (Utah Medical Products, Midvale, USA) designed to measure hydraulic pressure, and so the finer tubing on the mounting panel is flooded with sufficient water using the attached $3 \mathrm{ml}$ syringes. All interconnections are made with Med-Rx male/female plastic connectors and three-way junctions (Belan Inc., Oakville, Canada).

Functional hyperemia occurs in the several seconds following an increase in the action potential firing rate of a subpopulation of neurons. Because the action potential takes place over of a few milliseconds (see figure 1.4, page 9), it is possible to elicit an observable hemodynamic response for subsecond stimuli (Rosen et al., 1998). Hence, BOLD fMRI can resolve individual hemodynamic responses during an imaging session where the subject is exposed to brief stimuli separated by periods of inactivity. Experiments of this sort are known as event-related stimulus paradigms. As mentioned in the previous chapter, functional hyperemia is not an all-or-nothing phenomenon and will vary depending on stimulus duration and frequency of occurrence (Vazquez \& Noll, 1998). Figure 3.2 demonstrates this fact. Consequently, to elicit reproducible BOLD signals between stimuli, we decided to use a constant stimulus duration of two seconds, followed by a rest period lasting between 16 and 20 seconds. The exact duration of each rest period is randomly chosen before each session, so that the stimuli occur irregularly to minimise subject habituation and planning. A two-second stimulus duration was chosen because it 

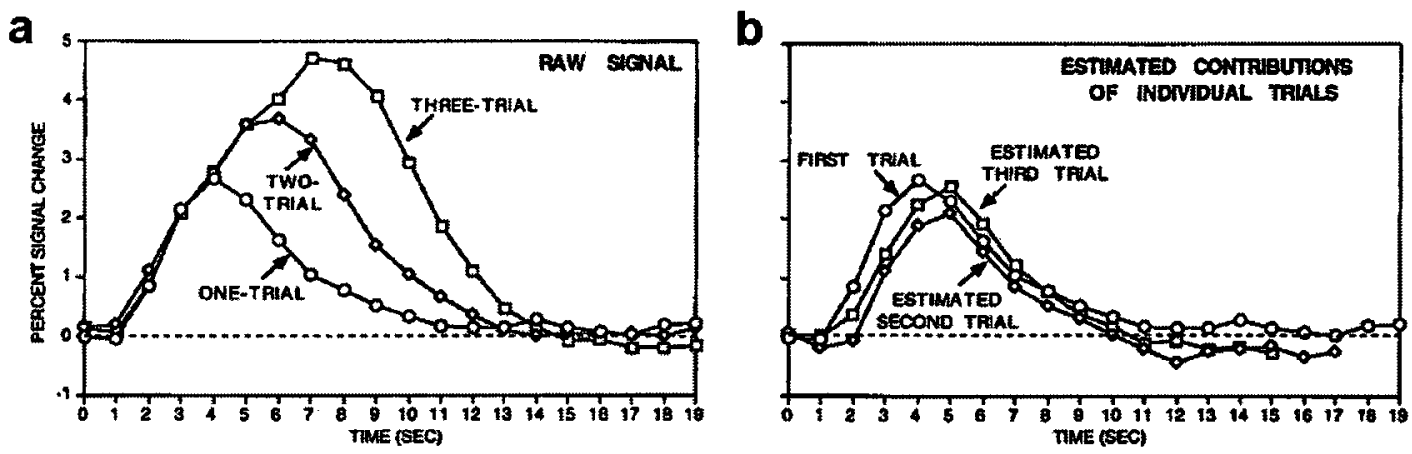

Figure 3.2: Plot of the BOLD signal measuring functional hyperemia after onesecond stimuli are presented once, twice, and thrice with one-second interstimulus rest periods, adapted from Rosen et al. (1998). a, The raw signals show that the hemodynamic response compounds with additional stimuli. b, Subtracting individual responses from the compound signal shows that subsequent responses are different from the original response.

coincides with the optimal fMRI $T_{R}$ value, i.e., the scanning repetition rate. This allows us to program event-related stimuli that are synchronised with the scanner $T_{R}$ cycle so that each hemodynamic response can be sampled consistently.

The nature of the stimulus and motor response are based on published methods used in other laboratories and on our research-specific objectives. We chose to stimulate the subject to produce a motor response using a visual cue that is displayed on a projection screen and visible through a small mirror mounted in front of the subject's eyes while inside the scanner. The display elements are kept to a minimum appearing on a flat dark blue background so as to limit the presentation of visual information. The elements visible on the display at all times are a fixation point in the centre of the display and two horizontal bars, a yellow one representing the paradigm state, and a light blue one representing the current grip force exerted by the tested hand. During the resting state, the subject is trained to relax while looking at the fixation point. At this time, both bars appear near the bottom of the display. During the two-second event, the yellow bar jumps upward to $60 \%$ of the screen height. The subject is trained to respond by squeezing the ball in their tested hand so as to raise the light blue bar as quickly and accurately as possible to reach the yellow bar. Regardless of the hand tested, the signals from both hands are 


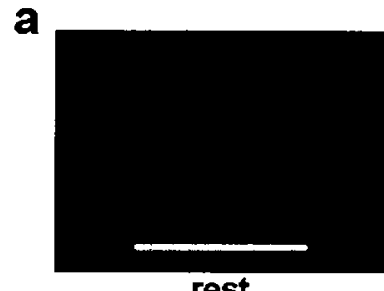

rest

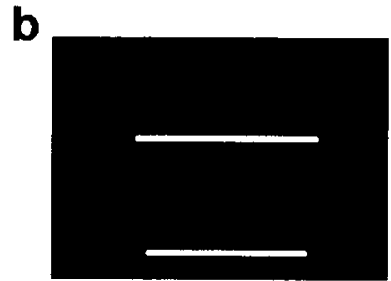

event

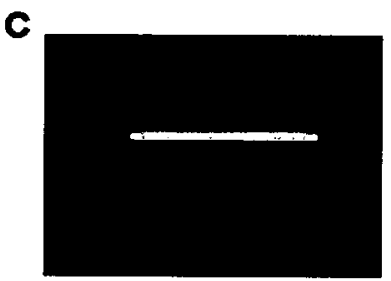

response

Figure 3.3: Display frames seen by the subject during rest (a), when an event begins (b), and when the subject performs the motor response (c).

always recorded. When the event ends, the yellow bar returns to its original position, and the subject is trained to relax their grip to allow the light blue bar to fall. The display during these states is shown in figure 3.3. The force required to reach the target is set to a fraction of the subject's maximum voluntary contraction (MVC), which is determined during training. To minimise ocular movements, the subject is trained to always look at the fixation point, and the screen is positioned to be in the centre of the subject's field of view, suotenaing a vertical angie of 5 degrees. Moreover, the position of the lignt biue bar is constrained so that it can never leave the display. As discussed in chapter 2 , it is known that target tracking motor tasks relying on visual feedback engage the various subcircuits of the sensorimotor system (see section 2.2.2, page 24). Furthermore, previous studies have reported results from similar approaches enabling interstudy comparison (Ward et al., 2003a,b; Turner et al., 2003).

The electronic portion of the apparatus driving the experiment was also implemented in our laboratory using LabView ver. 8.2 software-controlled electronics (National Instruments, Austin, USA). The electronics modules are shown in figure 3.4. During an imaging session, the apparatus is synchronised with the MR scanner $T_{R}$ cycle via a trigger pulse that is emitted by the scanner at the beginning of every image acquisition during the session. For a predetermined number of images to be acquired, the apparatus then generates events that always begin with a new image acquisition. The delay between the reception of a trigger pulse and the display update is kept under $10 \mathrm{~ms}$. Pressure transducer signals pass through an amplifier (World Precision Instruments, Saratosa, USA) 


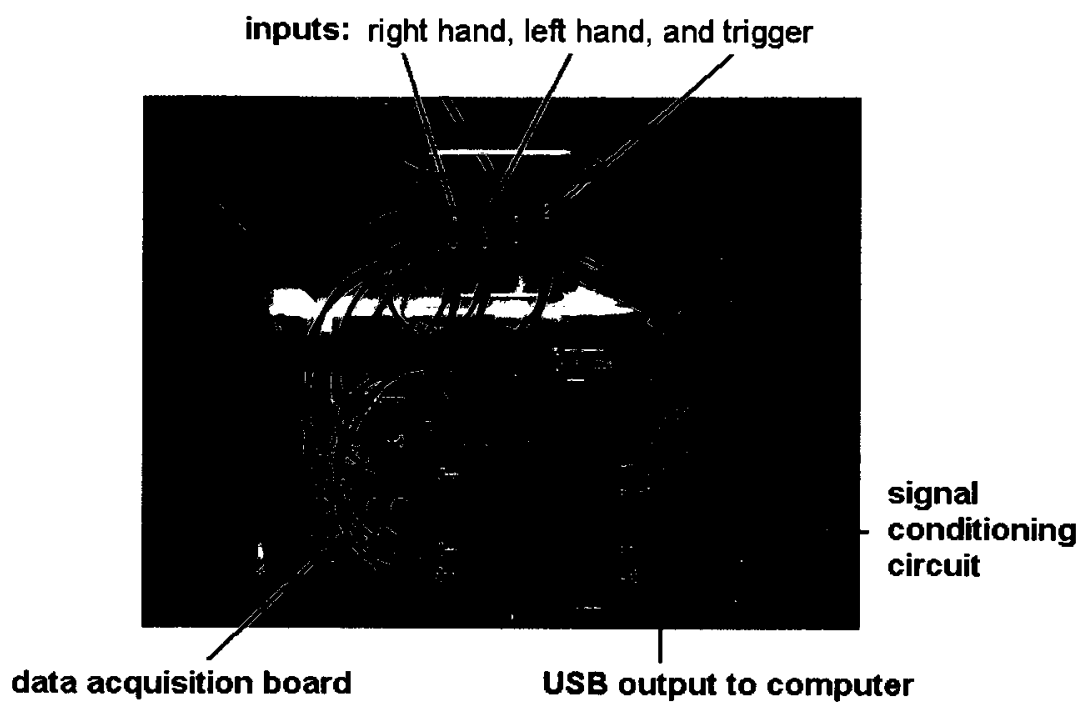

Figure 3.4: Photograph of the electronic portion of the experimental apparatus (top view). The signal inputs, the signal conditioning circuit, the data acquisition board, and the computer output are labelled.

before reaching our electronics. The signal conditioning circuit was necessary to isolate all signals from possible spikes in outlet power using a battery-powered voltage follower circuit, and to extend the trigger pulse from $10 \mu \mathrm{s}$ to $10 \mathrm{~ms}$ to make it compatible with the remainder of our circuit logic. The data acquisition hardware allows up to a $2 \mathrm{kHz}$ sampling rate on each motor signal.

The complete experimental apparatus is summarised in figure 3.5, where the right hand is being tested during this session. The apparatus records the trigger signal, the event timing, and the motor signals from both hands. The neuroimage data are acquired by the MR scanner and recorded on optical media via the scanner console.

\subsection{Neuroimaging data}

All image data are acquired using the Magnetom Symphony MRI scanner operating within a 1.5 Tesla magnetic flux density (Siemens, Erlangen, Germany). The gradient fields in this system have slope $30 \mathrm{mT} / \mathrm{m}$ and slew rate $125 \mathrm{~T} / \mathrm{m} / \mathrm{s}$. The radio frequency (RF) head coil system used consists of 16 circularly polarised phased array elements. The functional 


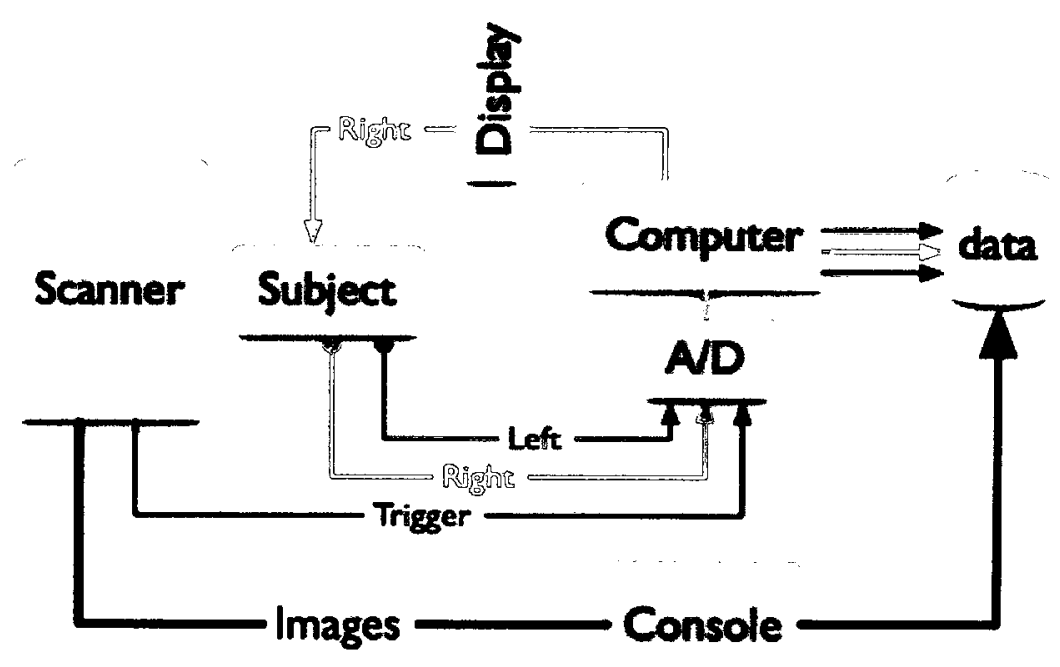

Figure 3.5: Diagram of the experimental apparatus interacting with the scanner and the subject ('A/D' represents the data acquisition board).

and structural imaging were performed using standard MR pulse sequences available with the scanner, and so only the pertinent details are given for each protocol.

The BOLD fMRI images were acquired using the Siemens echo-planar imaging (EPI) pulse sequence with name 'epfid2d1_64'. This is a $70^{\circ}$ gradient-echo pulse followed by the EPI pulse sequence with timing $T_{R}=2000 \mathrm{~ms}$ and $T_{E}=30 \mathrm{~ms}$. The interpolated voxel size is $1.72 \times 1.72 \times 5.00 \mathrm{~mm}^{3}$ along the read-encode, phase-encode, and slice select directions, respectively. Each image is formed from $128 \times 128 \times 26$ contiguous voxels along these same directions. The in-plane orientation, i.e., spanned by the readand phase-encoding directions, is set to acquire transversal sections of the brain that are parallel to the straight line segment formed by the anterior and posterior commissures (e.g., this plane would be a horizontal line passing through figure 1.2 on page 5). The field of view covers full transversal sections of the head, from the vertex of the skull down to just below the midway point of the cerebellum and medulla. Slices are collected in an interleaved fashion to minimise adjacent slice interference from RF residual excitation. With these parameters, we can collect 160 consecutive whole brain images. Hence, a single scan acquires the BOLD signal over $320 \mathrm{~s}$ with two-second resolution. The raw data points 
are encoded as unsigned 16-bit integers; thus, one session of raw fMRI data occupies 130 MB of memory.

In order to compare data between sessions and across subjects relative to an anatomical atlas, we decided to preprocess the raw data using the standardised software package SPM5 (Wellcome department of cognitive neurology, University College London, UK). Rigid body realignment is first performed, which helps to reduce minor head motion artefacts. We verifed that no major movements, i.e., having components greater than twice the corresponding voxel dimension, were present in the data after realignment. Second, slice time-interpolation is performed to correct for the systematic interslice acquisition delay so that the entire image can be treated as an instantaneous sampling of the BOLD signal taken midway through the pulse sequence. Third, each image is spatially normalised to fit the ICBM atlas using an affine transformation algorithm that estimates the transformation parameters from the mean realigned image, resulting in a resampled image of $101 \times 122 \times 28$ voxels. The trilinear interpolation technique used by this software package minimises the introduction of aliasing artefacts to tolerable levels when applied to realigned fMRI data Ashburner (2008). Finally, each image is isometrically smoothed using a Gaussian kernel of $8 \mathrm{~mm}$ full width at half its maximum value. While this kernel reduces the spatial resolution, it considerably improves the BOLD signal strength because of the multivoxel extent of functional hyperemia and accounts for the preprocessing errors introduced by anatomical differences between subjects. These preprocessing steps effectively reduce the session data size to $105 \mathrm{MB}$ of memory.

Structural imaging is also performed before the subject is removed from the scanner using a standard Siemens $T_{2}$ fluid attenuated inversion recovery (FLAIR) pulse sequence with name 'tir2d1_19' and parameters $T_{R}=9000 \mathrm{~ms}, T_{E}=99 \mathrm{~ms}$. The exact orientation parameters used for the functional imaging are re-used here so that both image types are co-registered in space. The affine transformation parameters used to normalise the functional images are applied to the structural image so that an anatomical reference for 
the BOLD data is possible.

\subsection{Participation of normal and stroke subjects}

The participation of human subjects in biomedical experimentation first requires the approval of the research institute's ethics board. Then, each individual must be informed of the details of their participation and in return give written informed consent of their willingness to be involved.

Permission to recruit healthy volunteers participating as normal subjects in this study was granted by the Ottawa Hospital Research Ethics Board (OHREB) as protocol \# 2009395-01H. Appendix A contains copies of the official letter of approval from OHREB and the completed application form, including the information letter and questionnaire. Suitable healthy volunteers must be between 20-65 years of age without contraindications to an MRI examination. Exclusion criteria are pregnancy, inability to perform the task, diabetes, uncontrolled hypertension, chronic obstructive pulmonary disease, congestive heart failure, or other potentially significant medical or surgical conditions. The subjects also must not have a history of neurological disease or drug/alcohol abuse, nor a seizure in the past year or migraine in the past week. The subjects are informed that they are free to withdraw from the study at any time.

The stroke patients are recruited under a different OHREB-approved multi-centre clinical trial (protocol \# 2006076-01H) for assessing the feasibility and safety of a drug treatment to augment stroke recovery. Each patient or their surrogate gave the informed consent to participate. The participating patients suffered an acute ischemic stroke involving the cerebral cortex resulting in hemiparesis and have no contraindications to an MRI examination. The patients do not have past or current additional complications, including those of neurological and cardiovascular nature, deemed significant according to standard clinical examination scores. Patients also have no ongoing history of drug/alcohol 
abuse. The BOLD fMRI data contribute to formulating the assessment of this treatment. While data acquisition and analysis were performed, the investigators were blinded to each patient's medication records.

The relevant functional neuroimaging procedures undertaken by both subject groups begins with a motor task training session of 10-20 minutes in duration. First, the MVC is measured from multiple trials for each hand, whence all subsequent force targets and responses can be calibrated as a percentile of MVC. The event-related stimulus paradigm training then takes place, appearing exactly as does in the scanner. The nominal force required to match the event is set to $25 \%$ MVC; however, patient-specific adjustments are possible. After training, the subjects undergo MRI, lying supine with their heads comfortably supported by movement restricting padding and wearing disposable earplugs underneath noise-dampening headphones. The hand grip devices are placed in their hands with arms comfortably lying alongside their body. A 5-minute test of the apparatus is done, which also ensures that the subject can see and respond to the events properly. The entire scanning procedure involves several BOLD fMRI sessions, testing both hands, and one $\mathrm{T}_{2}$ FLAIR scan. Normal subjects underwent a total of 4-6 fMRI scans for both hands, altogether spending 30-40 minutes in the scanner. Stroke patients underwent a total of 3-4 fMRI scans for both hands, altogether spending 25-30 minutes in the scanner. While scanning, an emergency signalling device was available to the subject if they wished to be immediately removed from the scanner. In addition, the subjects were also monitored by a closed circuit camera during the study to ensure that everything was fine. A study investigator was present during all the scans; no complications occurred with any of the subjects during these studies. 


\subsection{Discussion}

The results in the following chapters will show that this data acquisition method achieves its goals of inducing functional hyperemia reproducibly while simultaneously recording the nervous system response. Nonetheless, improvements to the proposed method are desirable. For example, the MR scanner is exceedingly loud, and its $\mathrm{T}_{R^{-}}$-rhythmic vibrations remain audible despite the noise-dampening precautions. Also, we are relying on a visual stimulus to test the sensorimotor system. Although we minimise the portion of the visual field involved by keeping the room dark and the display simple, the stimulus is nonetheless visual. Hence, the intersubject variability in vision quality becomes a factor because we use a projection system rather than MR-compatible display goggles. Generally speaking, one must remember that the living brain is a complex system whose function ultimately remains beyond the control of the experimenter. Therefore, in addition to the provoked response we seek, concurrent functions are also taking place, some being related to the task and others not. Another limitation is the lack of simultaneous neuronal response localisation. In some paradigms, an alternative would be to sacrifice simultaneity and use two sessions of functional neuroimaging, e.g., EEG/MEG followed by BOLD fMRI. However, for the purposes of this study, simultaneity is very important for correctly recognising the hemodynamic responses that are temporally related with the motor responses. Finally, this experimental paradigm focuses particularly on the sensorimotor system and, hence, is less sensitive to impairments in other faculties. Therefore, the observations made in this study are intrinsically limited to the effects of stroke on the sensorimotor system and should not be extended further.

Special considerations are necessary when working with stroke patients because their impairments and clinical needs vary considerably. Therefore, a flexible experimental protocol is required to enrol a good representation of the population. The scanner room must be as clear as possible for clinical staff and their equipment. A clinician must be present at all times in case of an adverse occurrence. On occasion, a nurse is present to 
provide care and assist the patient. Patients may also be using or require MRI-compatible medical equipment that must be accommodated in the scanner room. Thus, our apparatus only requires that the patient hold a hand grip device in each hand, and that their lineof-sight to the display remain clear during the functional imaging sessions.

When patients are required to perform tasks, they must be simple enough so that the majority can participate. Furthermore, despite the range of impairment, the perceived difficulty of the task should be constant across the group so as to elicit similar neuronal responses. Thus, the hand grip task in our method requires little dexterity, since the motion is gross involving the entire hand and, therefore, is easy to perform. To control for perceived difficulty, the target force is determined relative to the MVC of the tested hand, and in cases of severe paresis, exceptional adjustments are possible.

During scanning, patients typically exhibit more head motion than normal subjects. Despite the use of our head-restraining padding, movements of several millimetres can still occur. Images with large movements produce prominent artefacts in the neuroimage data that often lead to their exclusion in analysis. This remains a difficult challenge in MR data acquisition, since BOLD signal detection is already non-trivial without prominent motion artefacts. Secondly, patients sometimes perform irregular responses, e.g., hand contractions without stimulus, or failure to respond during some stimuli, or responses with the wrong hand, or with both hands. Irregular responses are also a challenge, since they introduce additional variability in the data, yet they are likely relevant to the task and, hence, should not be ignored. For example, one should not assume that responses performed by both hands simultaneously will elicit a neuronal activity pattern that necessarily corresponds to the superposition of two separate single-hand responses. Thus, our apparatus always records the motor signals in both hands in order to detect irregular responses and analyse them accordingly. In our analyses, we analysed neuroimage data acquired during two-handed movements by performing three analyses with different motor references: two using the left and right hand signals, and one using a superposition of both hand signals. 


\section{Chapter 4}

\section{Data Acquisition Methods: Part II Preliminary Analysis and Data Simulation}

\subsection{Introduction}

Given the acquired data described in the previous chapter, we face the problem of detecting the effect of functional hyperemia in BOLD fMRI data. Section 4.2 provides a preliminary analysis of our image acquisition method with the objective of modelling the noise in our data as a random process. The noise model can then be characterised by its mean and autocovariance functions, which are used to estimate the correlation between voxels in space and time.

This approach is used to model both scanner and physiological noise process. For scanner noise, data are acquired from a cylindrical plastic tank containing saline water, referred to as a "phantom." For physiological noise, we acquired null fMRI data from normal subjects; i.e., they underwent the experimental protocol of section 3.2 except without stimuli. All data were acquired using the same functional neuroimaging protocol from section 3.3, with the exception that the phantom data did not undergo spatial normalisation. These functions serve two purposes: i) to select the RF coil settings that minimise the intervoxel correlations while retaining good image contrast, and ii) to help reproduce the 
instrumental and physiological noise process in data simulation.

Secondly, section 4.3 characterises the functional hyperemia signal by its temporal profile following the stimulus event. To do this, we shall survey the signal characteristics observed in normal subjects and patients with CVD that have been reported in the literature. These temporal characteristics will be useful for the interpretation of the signals detected in our neuroimage data and, combined with the noise properties, will allow us to generate physiologically reasonable simulations.

Two simulation methods are designed based on these results in section 4.4. The first method generates synthetic data to test the signal detection performance under various signal-to-noise ratio (SNR) conditions and the capability to distinguish between multiple signals. The noise process is reproduced from the phantom data. The second uses a block resampling method for correlated data as per ? to generate samples from the pool of null fMRI sessions. The functional hyperemia signals are then added to these simulations.

These simulations will be used as the performance benchmark to analyse and compare the signal detection algorithms used in the following chapters. Finally, the analysis and simulation methods used here are discussed in relation to those found in the literature.

\subsection{Mean and autocorrelation function estimation}

The noise data are modelled as a real-valued random process, where each voxel measurement $w$ is a realisation of the random variable $\mathbf{w}$. Then, the time sequence of measurements in a voxel $w[t]$ for $t=0, \ldots, T-1$ is formed by the realisation of a sequence of random variables $\mathbf{w}_{t}$ called a random (or stochastic) process. Similarly, to model an image formed by a set of $X$ voxels, the time index can be substituted by the voxel coordinates written as the multivariate $x \in \mathbb{R}^{3}$. Hence, $w[x]$ is one noise image realised by the random process $\mathbf{w}_{x}$. Finally, a sequence of images is represented by the generalisation $w[n]$ realised from $\mathbf{w}_{n}$, where $n=[x, t]$. This indexing notation is used throughout the thesis to unambiguously 
refer to time sequences, images, or image sequences.

The mean and autocorrelation functions, respectively written as $\mu[n]$ and $\phi[n, m]$, are useful to study the correlation structure of $\mathbf{w}_{n}$. They are defined as

$$
\begin{aligned}
\mu[n] & =\mathcal{E}\left\{\mathbf{w}_{n}\right\}=\int_{-\infty}^{\infty} w p_{n}(w) d w \\
\phi[n, m] & =\mathcal{E}\left\{\mathbf{w}_{n} \mathbf{w}_{m}\right\} \\
& =\int_{-\infty}^{\infty} \int_{-\infty}^{\infty} w v p_{n m}(w, v) d w d v
\end{aligned}
$$

where the expectation operator $\mathcal{E}\{\cdot\}$ averages over the ensemble of realisations of $\mathbf{w}_{n}$, equivalently represented in these equations by the probability density function $p_{n}(\cdot)$ of $\mathbf{w}_{n}$ and the joint density function $p_{n m}(\cdot, \cdot)$ of the ordered pair $\left(\mathbf{w}_{n}, \mathbf{w}_{m}\right)$ (Papoulis, 1984). The correlation coefficient $r[n, m]$ quantifying the dependence between variables $\mathbf{w}_{n}$ and $\mathbf{w}_{m}$ is obtained by calculating the autocorrelation between the mean-centred random processes $\mathbf{w}_{n}-\mu[n]$ and $\mathbf{w}_{m}-\mu[m]$, called the autocovariance function (Oppenheim \& Schafer, 1999)

$$
\begin{aligned}
\gamma[n, m] & =\mathcal{E}\left\{\left(\mathbf{w}_{n}-\mu[n]\right)\left(\mathbf{w}_{m}-\mu[m]\right)\right\} \\
& =\mathcal{E}\left\{\mathbf{w}_{n} \mathbf{w}_{m}\right\}-\mu[n] \mu[m] \\
& =\phi[n, m]-\mu[n] \mu[m] .
\end{aligned}
$$

Indeed, by applying equation (4.3) to the definition of the correlation coefficient, we obtain

$$
\begin{aligned}
r[n, m] & =\frac{\mathcal{E}\left\{\left(\mathbf{w}_{n}-\mu[n]\right)\left(\mathbf{w}_{m}-\mu[m]\right)\right\}}{\sigma[n] \sigma[m]} \\
& =\frac{\gamma[n, m]}{\sqrt{\mathcal{E}\left\{\left|\mathbf{w}_{n}-\mu[n]\right|^{2}\right\} \mathcal{E}\left\{\left|\mathbf{w}_{m}-\mu[m]\right|^{2}\right\}}} \\
& =\frac{\gamma[n, m]}{\sqrt{\gamma[n, n] \gamma[m, m]}},
\end{aligned}
$$

where $\sigma[n]=\sqrt{\mathcal{E}\left\{\left|\mathbf{w}_{n}-\mu[n]\right|^{2}\right\}}$ is the standard deviation (SD) of $\mathbf{w}_{n}$.

When a large ensemble of realisations $\{w[n]\}$ is not available, and the densities 
$p_{n}(\cdot)$ and $p_{n m}(\cdot, \cdot)$ are unknown, it is often acceptible to estimate equations (4.1)-(4.3) by assuming that $\mathbf{w}_{n}$ is an ergodic random process. When this property holds, the expectation $\mathcal{E}\left\{\mathbf{w}_{n}\right\}$ obtained by the ensemble average of $\{w[n]\}$ can instead be calculated by averaging the indexed measurements from a single realisation $w[n]$. Hence, the estimate of the mean in equation (4.1) is simply the average of our finite data set $w[n]$, written as

$$
\hat{\mu}[n]=\langle w[n]\rangle=\frac{1}{N} \sum_{n=0}^{N-1} w[n] .
$$

The notation $\langle\cdot\rangle$ represents the index average and replaces the ensemble average $\mathcal{E}\{\cdot\}$ in ergodic estimates. Of course, this equation can only be valid if $\mu[n]=\mu$ is a constant function over $n$. Because we are also interested in the statistics along $t$ and $x$, we can also estimate the marginal means $\hat{\eta}[x]$ and $\hat{\eta}[t]$ along the space and time dimensions, respectively. For example, $\hat{\eta}[t]$ averages over space yielding the time series

$$
\begin{aligned}
\hat{\eta}[t] & =\langle w[n]\rangle_{x} \\
& =\frac{1}{X} \sum_{x=0}^{X-1} w[x, t],
\end{aligned}
$$

where it is understood that the summation applies to all three spatial dimensions.

Ergodicity implies that the autocorrelation function $\phi[n, m]$ takes on the same values when $\|n-m\|=\tau$ is constant (Papoulis, 1984). Hence, the autocorrelation reduces to a one-parameter function $\phi[\tau]$, invariant to the absolute coordinates $n$ and $m$. A random process satisfying the properties $\mu[n]=\mu$ and $\phi[n, m] \rightarrow \phi[\tau]$ for all $n$ is called wide-sense stationary (WSS). Hence, for a WSS random process $\mathbf{w}_{n}$, it follows from $\mu[n]=\mu$, that $\phi[\tau] \rightarrow 0$ as $\tau \rightarrow \infty$. The ergodic estimate of $\phi[\tau]$ for a finite $N$ is

$$
\begin{aligned}
\hat{\phi}[\tau] & =\langle w[n] w[n+\tau]\rangle \\
& =\frac{1}{N} \sum_{n=0}^{N-1} w[n] w[n+\tau],
\end{aligned}
$$


where $w[n]=0$ for $n<0$ and $n \geq N$ (Oppenheim \& Schafer, 1999). From the last line of this equation, we see that $\hat{\phi}[\tau]$ is a function of size $2 N-1$ that is symmetric about the origin, hence $\hat{\phi}[-\tau]=\hat{\phi}[\tau]$. Consequently, this estimate is equal to the linear convolution of $w[n]$ with itself, which is efficiently computed using the discrete Fourier transform (DFT) of $w[n]$, defined as (Oppenheim \& Schafer, 1999)

$$
W[k]=\mathscr{F}\{w[n]\}=\sum_{n=0}^{N-1} w[n] e^{-i 2 \pi k n / N}
$$

It is also worthwhile to recall that the DFT implementations are most efficient when $\sqrt{N}$ is an integer, and so the original set can be zero-padded to smallest square integer satisfying $Q>2 N$. This can be shown by beginning with the DFT of $\hat{\phi}[\tau]$ for $\tau=0, \ldots, Q-1$

$$
\hat{\Phi}[k]=\mathscr{F}\{\hat{\phi}[\tau]\}=\sum_{\tau=0}^{Q-1} \hat{\phi}[\tau] e^{-i 2 \pi k \tau / Q}
$$

and replacing $\hat{\phi}[\tau]$ with the convolution form in equation (4.7). This yields

$$
\begin{aligned}
\hat{\Phi}[k] & =\sum_{\tau=0}^{Q-1}\left(\frac{1}{Q} \sum_{n=0}^{Q-1} w[n] w[n+\tau]\right) e^{-i 2 \pi k \tau / Q} \\
& =\frac{1}{Q} \sum_{n=0}^{Q-1} w[n]\left(\sum_{\tau=0}^{Q-1} w[n+\tau] e^{-i 2 \pi k \tau / Q}\right) .
\end{aligned}
$$

The term in brackets equals the DFT of $w[\tau]$ shifted by $n$, namely $W[k] e^{i 2 \pi k \tau / Q}$, which can be conveniently rewritten by replacing $k$ with $-k$. By making these substitutions and regrouping terms, the result is obtained

$$
\begin{aligned}
\hat{\Phi}[k] & =\frac{1}{Q} \sum_{n=0}^{Q-1} w[n]\left(W[-k] e^{-i 2 \pi k n / Q}\right) \\
& =\frac{1}{Q} W[-k] \sum_{n=0}^{Q-1} w[n] e^{-i 2 \pi k n / Q} \\
& =\frac{1}{Q} W[-k] W[k] .
\end{aligned}
$$


By using equation (4.11), the autocorrelation estimate can be recovered with the inverse DFT. Finally, applying the symmetry property of $\hat{\phi}[\tau]$, the forms for $\hat{\phi}[\tau]$ and $\hat{\gamma}[\tau]$ reduce to

$$
\begin{aligned}
& \hat{\phi}[\tau]=\frac{1}{Q^{2}} \sum_{k=0}^{Q-1} W^{2}[k] e^{i 2 \pi k \tau / Q}, \\
& \hat{\gamma}[\tau]=\frac{1}{Q^{2}} \sum_{k=0}^{Q-1} W^{2}[k] e^{i 2 \pi k \tau / Q}-\hat{\mu}^{2} .
\end{aligned}
$$

The validity of the ergodic and WSS assumptions can only be determined by the physical interpretation of the system and by the examination of the data. In fact, the noise on the MR signal is primarily due to electronic sources (known as Johnson noise) and from the dielectric and inductive coupling with the body. For these reasons, it is typically considered ergodic and stationary (Cardenas-Blanco et al., 2008). The empirical results from our data support these interpretations. Using data from six phantom imaging sessions taken on separate days, the estimates $\hat{\mu}[n], \hat{\eta}[x]$, and $\hat{\eta}[t]$ were calculated from equations (4.5) and (4.6) to verify the ergodicity of $\mathbf{w}_{n}$. While there was considerable intersession variability between the mean intensity of the image $\hat{\mu}[n]=1688 \pm 223$ au (arbitrary units; mean $\pm \mathrm{SD}$ shown), each function $w[n]$ was flat with variance $\hat{\sigma}^{2}[n]=2.2 \pm 0.3 \mathrm{au}^{2}$. In fact, our data did not deviate from the mean by more than $0.25 \%$ in any direction. The autocovariance estimate $\hat{\gamma}[\tau]$ was calculated from equations (4.12) and (4.13) using five non-overlapping rectangular subsets of $w[n]$ of size $N=X T=16^{3} \times 16$ : sampled half-way through the session and located i) in the image centre, then further along ii) the read-encode axis $x$, iii) the phase-encode axis $y$, iv) the slice-select axis $z$, and v) back in the centre but later in time. The $\hat{\gamma}[\tau]$ function is normalised and plotted along the four axes in figure 4.1. Indeed, the estimate smoothly tends to zero as $\tau$ gets large and remains relatively constant throughout the data set with correlation coefficients $r[n, m]$ for adjacent and subsequent voxel measurements being $r[x, x+1]=0.82 \pm 0.008, r[y, y+1]=0.80 \pm 0.02$, $r[z, z+1]=0.64 \pm 0.03$, and $r[t, t+1]=0.21 \pm 0.08$ (mean $\pm \mathrm{SD}$ shown $).$ 

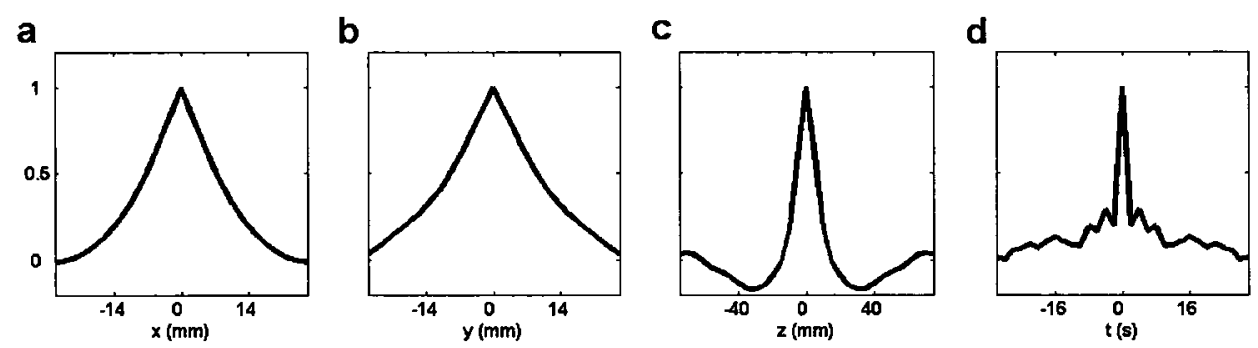

Figure 4.1: The autocovariance function estimate $\hat{\gamma}[\tau]$ plotted along the read-encode $x$-axis (a), the phase-encode $y$-axis (b), the slice-select $z$-axis (c), and the time axis (d). The phantom data were acquired in the CP (PN on) RF coil setting.

These results clearly show that our fMRI data are quite correlated. We ensured that these were the smallest correlations possible by comparing results with other applicable RF coil settings available on the scanner. The correlation analysis was done for the available RF coil settings labelled 'Circular Polarisation' (CP), 'Dual', and 'Triple', where each can be preceded by a 'Prescan Normalisation' (PN) pulse, resulting in six possible settings. Furthermore, the SNR was compared between settings defined by $\mathrm{SNR}=20 \log _{10}(\hat{\mu} / \hat{\sigma})$ in decibels. The results are summarised in figure 4.2, showing that the CP setting with PN is optimal, yielding the lowest correlations with a competitive SNR of $19.4 \mathrm{~dB}$. Therefore, all scans performed on human subjects were done with the $\mathrm{CP}$ ( $\mathrm{PN}$ on) RF coil setting.

\subsection{Physiological BOLD signal characterisation}

The terms "BOLD signal" or "BOLD response" are used henceforth to refer to the transient fluctuation in the fMRI data caused by functional hyperemia. As described in section 1.4.4, the normal BOLD signal is an increase in the MR signal intensity due to the disproportional increase $\triangle \mathrm{CBF}>\Delta \mathrm{CMRO}_{2}$ causing a reduction in concentration of paramagnetic deoxy-Hb. The temporal characteristics of the BOLD signal, such as its amplitude and duration, will depend on both the stimulus and the physics of MRI, in particular, the magnetic flux density and RF pulse sequence. Consequently, the characterisation presented here is focused on our data acquisition approach: event-related stimuli during fMRI 

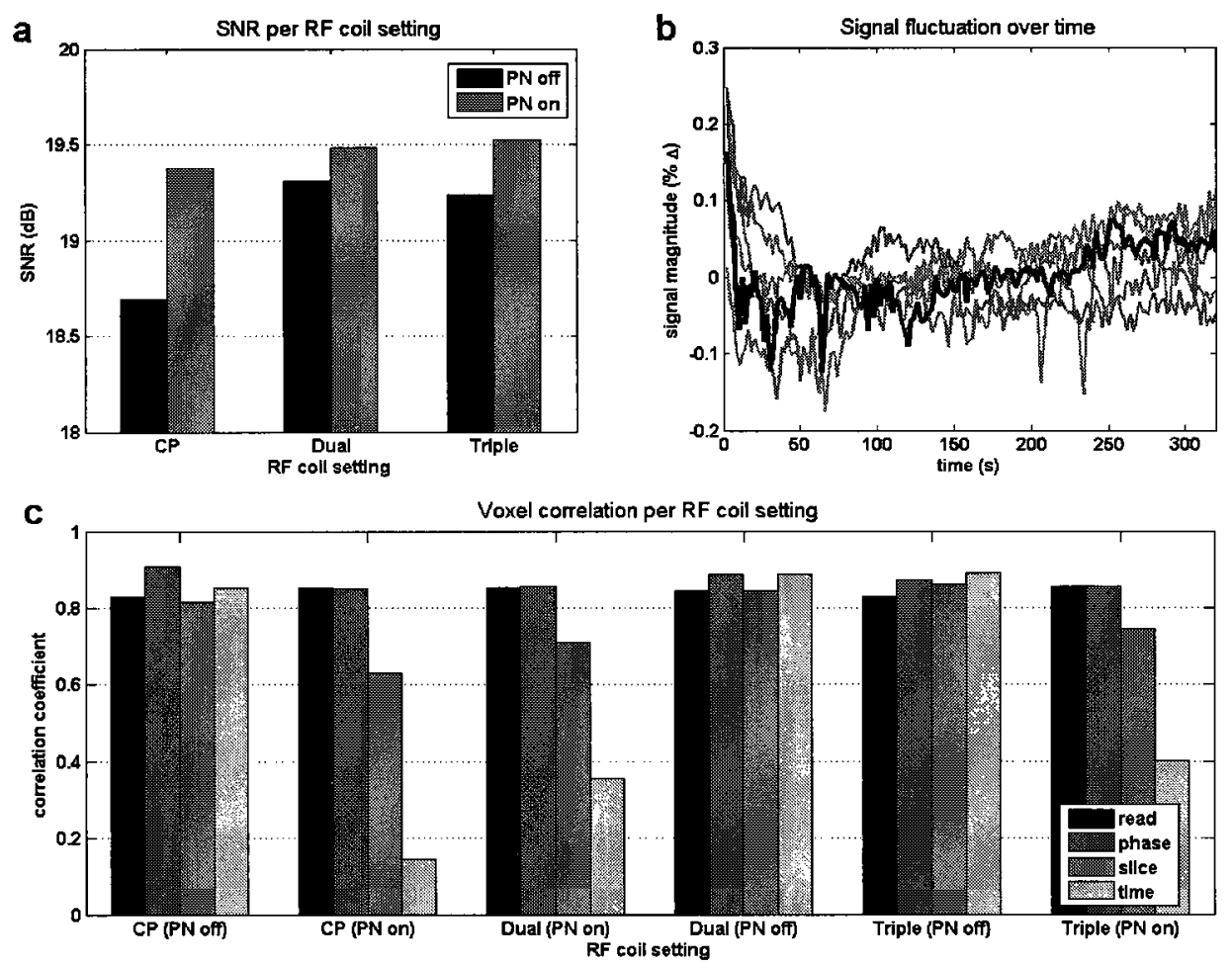

Figure 4.2: Comparison of the six possible MRI RF coil settings. a, The SNR is shown in $\mathrm{dB}$. b, Plot of the six $\hat{\eta}[t]$ signals shown as percent change from the mean; the heavy black curve corresponds to $\mathrm{CP}$ ( $\mathrm{PN}$ on). c, The adjacent intervoxel correlation coefficients along the read-encode $x$, phase-encode $y$, slice-select $z$, and time $t$ axes. 
at $1.5 \mathrm{~T}$ using gradient echo-EPI acquisition.

The paramagnetic effect of [deoxy-Hb] causes only a minor change in the MR physics of the cerebrovasculature. More specifically, within a voxel, it marginally increases the MR transverse relaxation rate of the average proton magnetisation that is dominated by the $\mathrm{T}_{2}^{*}$ decay term (Buxton et al., 2004). For this reason, the BOLD signal represents less than $5 \%$ of the voxel average intensity (e.g., see figure 3.2, page 39). The BOLD signal in the SMC evoked by event-related stimuli is on the order of a $1 \%$ change from the average intensity, which is written henceforth as $1 \% \Delta$. When comparing this amplitude to the background noise, various SNR definitions are used throughout the literature. We selected the most common from twelve relevant articles being

$$
\mathrm{SNR}_{\mathrm{BOLD}}=20 \log _{10}\left(\frac{A}{\sigma}\right)
$$

that is, the BOLD signal amplitude divided by the noise standard deviation. With this definition, the typical range for BOLD SNR is $[-12,6.0] \mathrm{dB}$ corresponding to the $A / \sigma$ ratio of $[0.25,2.0]$. Therefore, a reasonable estimate of SMC event-related BOLD signal quality in $1.5 \mathrm{~T}$ MRI is a $1 \% \Delta$ signal corrupted by noise with $\sigma \approx 0.25 \% \Delta$. A review of how the BOLD SNR depreciates in the elderly and CVD populations is given by D'Esposito et al. (2003). In particular, Huettel et al. (2001) show in a study of age-related hemodynamics that the BOLD SNR in the elderly can be $50 \%$ of that in young participants. Pineiro et al. (2002) show that patients with major arterial stenoses can have BOLD SNR reductions on the order of $30 \%$ of that in normal subjects.

The normal BOLD signal can be divided into three post-stimulus phases: i) a delay before the response onset, ii) a positive peak width, and iii) a negative undershoot period after the response. In CVD patients, the duration and polarity of these phases change. Figure 4.3a plots three BOLD signals normalised to unit extrema whose characteristics are based on those described in the literature. Signal 1 represents a typical BOLD response in normal subjects. The selected values for the onset delay (1.3 s), time-to-peak (5 s), 

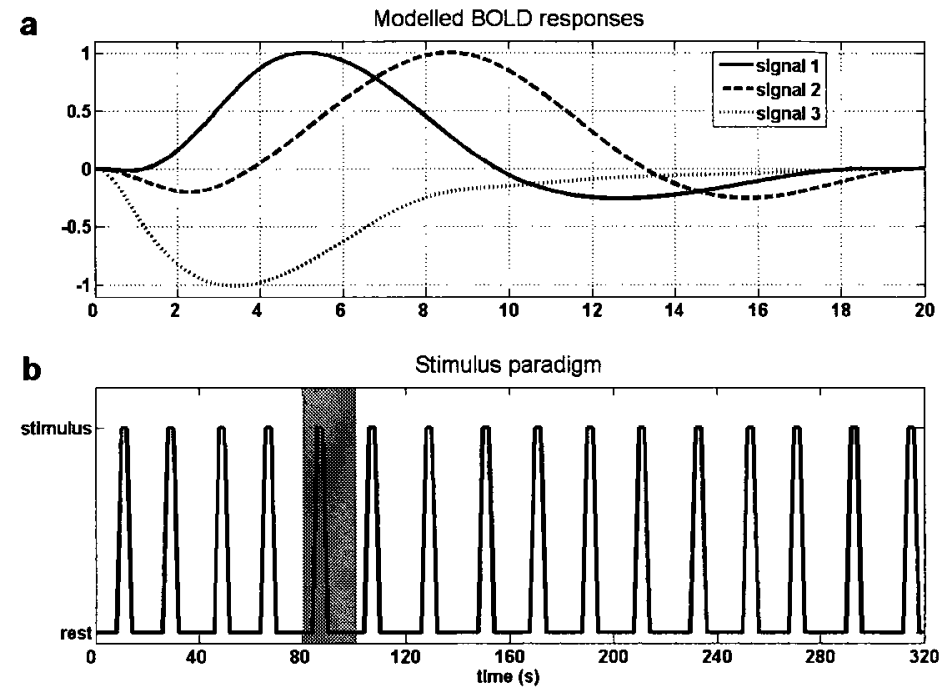

Figure 4.3: Simulated fMRI data. a, Modelled BOLD responses from normal subjects (signal 1) and CVD patients (signals 2,3). b, Event-related paradigm with interstimulus period randomly varying between 16-20 s (grey area indicates simulated BOLD response duration).

peak width $(8.3 \mathrm{~s})$, and undershoot duration (11 s) are the average of reported values from Friston et al. (1998), Huettel et al. (2001), and Buxton et al. (2004). Signal 2 is a delayed response that has been observed in the presence of CVD. Studies by Carusone et al. (2002) and Roc et al. (2006) have an average onset delay of $3.8 \mathrm{~s}$, and Roc et al. (2006) describe an early trough before onset. This trough is also referred to as the "initial dip" in the literature, which is attributed to an initial stage where an increase in $\mathrm{CMRO}_{2}$ has not yet been met by a concomitant increase in CBF (Röther et al., 2002; Buxton et al., 2004; Burke \& Bührle, 2006). Signal 3 is an advanced, negative peak response from CVD patients described by Röther et al. (2002) and Roc et al. (2006) where the time-topeak was advanced by $2 \mathrm{~s}$. These two cases represent unusual conditions where the CVD patients participated with a completely occluded internal carotid artery thereby severely disrupting functional hyperemia. 


\subsection{Data simulations}

The BOLD signal and noise properties were used to design two simulations for algorithm performance analysis.

The first simulation exclusively tests the detection accuracy of the three BOLD signals shown in figure 4.3 under SNR conditions ranging from $[-12,6.0] \mathrm{dB}$. Here, the signal locations are not in an anatomical arrangement. Instead, the signals are added to a central block of the image volume with $\mu[n]=1600$ au, each confined to one of three rectangular regions whose SNR decreases linearly moving upwards along the $y$ axis. The remainder of the image volume consists of a low intensity background without the BOLD signal and with noise generated by an independent Gaussian process convolved with $\hat{\gamma}[\tau]$ from phantom data. The purpose of this simulation is to provide a benchmark description of the algorithm's capability to detect, and distinguish between, the three signals as a function of SNR.

The second simulation uses a computer-intensive bootstrap method called block resampling, described by ?, in order to approximate the spatial and temporal properties of null fMRI data. The purpose of this approach is in large part a first attempt at generating anatomically realistic simulations without a BOLD response that estimate the correlation structure of real resting data. Upon these, one can then add a precisely controlled BOLD response signal with known location with SNR. The main advantage of bootstrap simulation is that a large number of sessions can be generated, hence, allowing an assessment of the algorithm's variability in performance across realistic noise realisations.

The block resampling approach goes as follows. Let $\left\{w^{(j)}[n]\right\}$ for $j=1, \ldots, J$ be an ensemble of $J$ null fMRI sessions. Each session will be partitioned into $k$ blocks of size $b$ of type $B_{i}=\left(w^{(j)}[i], \cdots, w^{(j)}[i+b-1]\right)$ so that each session has the form $w[n]=\left(B_{1}, B_{b+1}, \ldots, B_{N-b+1}\right)$ for $n=0, \ldots, N-1$. The bootstrap session $w_{b}^{*}[n]$ is defined piecewise by its contiguous sub-blocks of type $B_{i}^{*}$ each being drawn with replacement from the pool of $J \times k$ session sub-blocks. In this simulation, the anatomy is preserved in the 
simplest fashion: by not resampling along the spatial directions. Thus, each image is kept intact, and so the ensemble and bootstrap session can be written as time series $\left\{w^{(j)}[t]\right\}$ and $w_{b}^{*}[t]$ for $t=1, \ldots, T$.

It is important to choose a suitable block size. If $b$ is chosen too small, then $w_{b}^{*}[t]$ will have an underestimated autocovariance function; i.e., $\hat{\gamma}^{*}[\tau] \ll \hat{\gamma}[\tau]$ for $\tau>0$. On the other hand, if $b$ is chosen too large, then $w_{b}^{*}[t]$ will be biased toward the few sessions that were sampled rather than uniformly representing all $J$ sessions. It was natural to use a criterion for choosing $b$ that is based on the autocovariance along the $t$-axis $\hat{\gamma}_{t}[\tau]$. Hence, we estimated $\hat{\gamma}_{t}[\tau]$ and set $b$ equal to the central peak width delimited by (and excluding) the first zero-crossing or local minimum on each side of $\tau=0$. In order to estimate the function $\gamma_{t}[\tau]$ with good precision, we must choose $T$ as large as possible to reduce the variance of $\hat{\gamma}_{t}[\tau]$. The fact that the estimate is consistent, i.e., the variance of $\hat{\gamma}[\tau]$ vanishes as $N$ becomes large, is difficult to show because the calculation $\operatorname{var}\{\hat{\phi}[\tau]\}$ is non-trivial (Oppenheim \& Schafer, 1999). However, for a WSS process $\mathbf{w}_{n}$, we can see that the random variable $\boldsymbol{\phi}_{\tau}=1 / N \sum_{n=0}^{N-1} \mathbf{w}_{n} \mathbf{w}_{n+\tau}$ has expectation $\mathcal{E}\left\{\boldsymbol{\phi}_{\tau}\right\}=1 / N \sum_{n} \mathcal{E}\left\{\mathbf{w}_{n} \mathbf{w}_{n+\tau}\right\}=\phi[\tau]$, which converges on the order of $O(1 / N)$. Thus, the square of $\phi_{\tau}$ should shrink faster with order $O\left(1 / N^{2}\right)$ and if unbiased will also converge to $\phi[\tau]$, which implies that $\operatorname{var}\left\{\phi_{\tau}\right\}$ will vanish in the limit $N \rightarrow \infty$. This approach is summarised as the following algorithm.

Algorithm 1 (Time series bootstrap simulation). Given an ensemble of time series data sets $\left\{w^{(j)}[t]\right\}$ for $j=1, \ldots, J$ each of length $T$ (zero-padded to the smallest power of two greater than $T$ ), do the following to generate a time series bootstrap simulation.

1. Calculate the autocovariance function estimate $\hat{\gamma}_{t}[\tau]$ using equation (4.13) from each data set in the ensemble, and set the block size $b=2 \arg _{\tau=\tau_{b}}\left\langle\hat{\gamma}_{t}[\tau]\right\rangle-1$, where $\tau_{b}$ is the first zero-crossing or first local minimum, whichever is closest to the origin.

2. If necessary, truncate each data set to length $T=k b$, where $k \in \mathbb{N}$ is as large as possible.

3. Partition each data set from the ensemble into $k$ blocks of size $b$ of type $B_{i}^{(j)}=\left(w^{(j)}[i], \cdots, w^{(j)}[i+b-1]\right)$ so that each session has the form $w^{(j)}[t]=$ $\left(B_{1}^{(j)}, B_{b+1}^{(j)}, \cdots, B_{T-b+1}^{(j)}\right)$ for $t=0, \ldots, T-1$.

4. Construct a time series bootstrap data set $w_{b}^{*}[t]$ by randomly drawing with replace- 

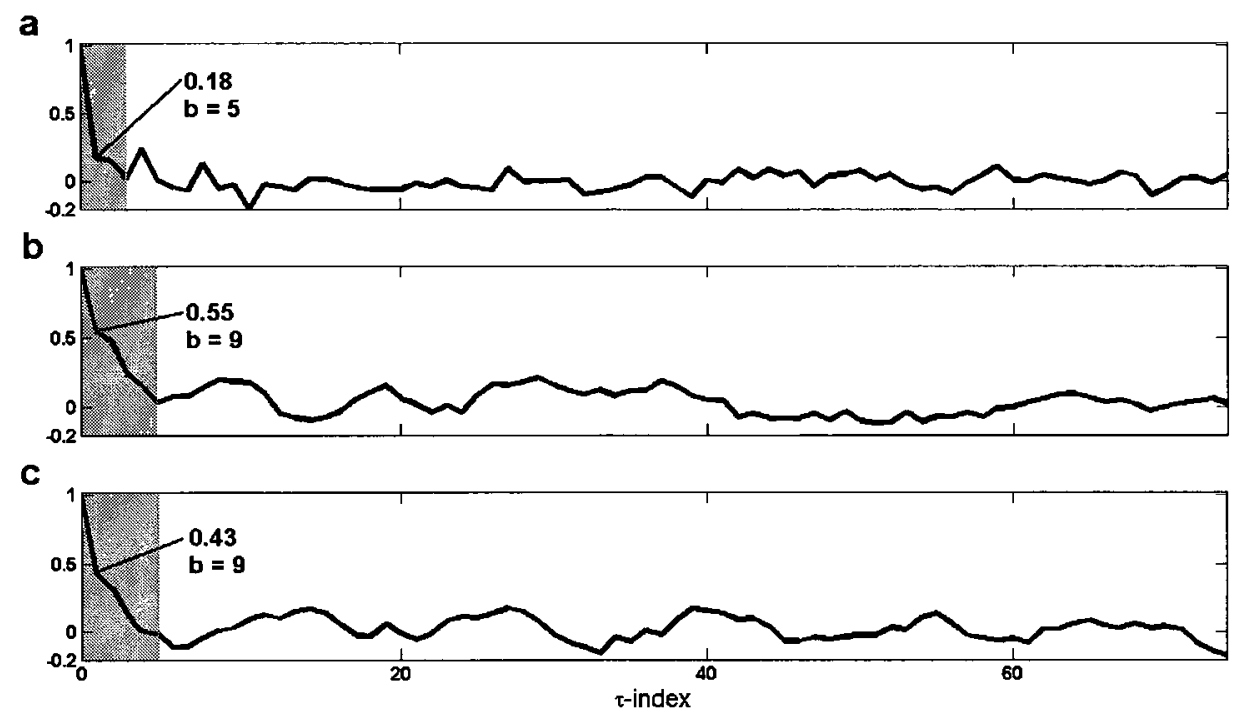

Figure 4.4: Typical autocovariance function estimates $\hat{\gamma}_{t}[\tau]$ computed from single voxel time series of length $T=150$ shown from head phantom (a), null fMRI data (b), and $b=9$ block resampling simulations from null fMRI data (c). The empirical correlation coefficient $\hat{r}[1]$ is shown along with the peak width shaded in grey.

ment $k$ blocks from the ensemble of $J \times k$ blocks $\left\{B_{i}^{(j)}\right\}$, for $j=1, \ldots, J$ and $i=1, b+1, \ldots, T-b+1$.

We chose $T=150$ by discarding the first ten images to avoid the presence of magnetisation stabilisation effects that cause a rapid drop in signal intensity in the several first images, e.g., as seen in figure 4.2b. For these calculations, we acquired $J=3$ consecutive sessions from a normal subject so that no repositioning took place between sessions. Five voxels, with fixed coordinates within the brain tissue but beyond $30 \mathrm{~mm}$ from each other, were selected to calculate $\hat{\gamma}_{t}[\tau]$ in for each session. The peak widths measured have median 9 and range $[7,13]$, and so we chose $b=9$. This leads to $k=17$ blocks per session and each bootstrap simulation is generated from random draws with replacement from the pool of $J \times k=51$ blocks. Our original attempt included session data acquired on different dates but were not amenable to the bootstrap simulation proposed because at the time of acquisition, the exact positioning of the subject within the scanner's field of view was not controlled and so block-related displacement artefacts corrupted the simulated data. 
For comparison, figure 4.4 plots typical autocovariance estimates, for $0 \leq \tau<75$ with their peak width shaded and $\hat{r}[1]$ correlation coefficient indicated, for the phantom, null fMRI, and simulated data. As expected, the phantom data are less correlated with narrower peak width than the null fMRI data. Secondly, the null data and simulation data are similar, possessing wide peaks and slowly oscillating sideband, in contrast to the narrow peak and jagged sideband in the phantom data. One consistent bias is that the simulated data tend to have slightly lower $\hat{r}[1]$ values than the null fMRI data. Perhaps, this is due to the disruption of spatial correlations and "second-order effects," i.e., those depending on higher-order interactions, such as in the ordered triple $\left(\mathbf{w}_{n}, \mathbf{w}_{m}, \mathbf{w}_{p}\right)$ between blocks.

\subsection{Discussion}

These preliminary analyses sought to characterise the underlying noise processes from our empirical phantom and null fMRI data. The autocorrelation estimates performed here show that GE-EPI voxel data are indeed correlated in space and time. It is also clear from these results that there are instrumental and physiological sources introducing temporal correlation in the data. In addition, high spatial correlations are introduced by anatomically normalising and spatially smoothing the fMRI data. The presence of these correlations has been studied and reported by many authors (e.g., see discussions in Zarahn et al. (1997); Purdon \& Weisskoff (1998)) and remains a major obstacle in neuroimaging analysis. This difficulty arises essentially in the calculation of statistical significance on dependent data sets.

For example, classical statistics such as the student's $t$ or Fisher's $F$ upon which hypothesis testing using linear regression and analysis of variance (ANOVA) assume that individual measurements are independent and identically distributed (i.i.d.). Hence, BOLD signal detection on a voxel-wise basis leads to an increased false-positive rate (or equiva- 
lently, an increased type I error rate, or false rejection of the null-hypothesis). Therefore, extraneous mechanisms must be introduced to account for the departure from the i.i.d. assumption to achieve the nominal false-positive rate. Furthermore, resampling statistical methods such as the bootstrap are also intended for i.i.d. data. Here too, the dependency between measurements complicates the efficient and consistent estimation of even the simplest statistics such as the standard error (Politis, 2003).

Zarahn et al. (1997) performed an empirical analysis on phantom and null fMRI data to demonstrate that the data are temporally correlated, and hence have a non-flat power spectrum density (PSD). They showed that the data PSD followed a $1 / f$ magnitude that was not consequent to first-order motion, instrumental, or preprocessing effects. Purdon \& Weisskoff (1998) pointed out that this is more conventionally understood as $1 / \omega^{2}$ PSD characteristic, which corresponds to a linear time-invariant (LTI) system, with a rational transfer function. Our data supports these observations, since there is a clear increase in temporal autocorrelation in null fMRI data compared to phantom data as seen in figure 4.4. In addition to temporal autocorrelation, Zarahn et al. (1997) go on to demonstrate the presence of a "global signal" in the sense that voxel time sequences $w\left[x_{1}, t\right]$ and $w\left[x_{2}, t\right]$ also tend to be strongly correlated for most coordinates $x_{1}, x_{2} \in \mathbb{R}^{3}$. The global signal also introduces a bias in hypothesis-test statistics or correlation estimates. Hence, signal detection methods must account for its effect. Consequently, we defer the global signal phenomenon to the signal detection chapters later in the thesis.

Recent efforts have applied autoregressive (AR) models to dependent data (including fMRI) such as to model the order of dependence in data and also to produce i.i.d. samples from the data. An effective AR model for fMRI data is very appealing for it would reduce the signal detection problem to well-established classical or bootstrap statistical analysis. These methods are not wide spread in fMRI analysis; however, some worthwhile examples of AR models for fMRI data have been published (Harrison et al., 2003; de Pasquale et al., 2008). Although this subject is not dealt with further in this 
thesis, it is an emerging topic in the literature which should be considered in future work.

Besides the mathematical issues arising from the analysis of correlated data sets, a surprising number of articles investigating novel neuroimage analysis methods are tested on oversimplified simulations that do not reproduce the reported features of real fMRI data, such as noise autocorrelation, BOLD signal characteristics, SNR levels, preprocessing, and artefacts. While the objective of the article may be to propose a formidable analysis method (and not detailed simulations), unrealistic simulations make it difficult to gauge the algorithm's performance on real data often lacking ground truth or a "gold standard."

To address these problems, we propose a bootstrap simulation algorithm based on real neuroimage data that preserves the observed correlation structure. However, the effectiveness of this method depends on the precision of subject positioning within the scanner so that displacement artefacts do appear in the intersession resampled blocks. At the time of acquisition, we did not control for this effect with sufficient precision and were only able to produce bootstrap samples using single subjects where the subject remained in the scanner between consecutive sessions.

An autocorrelation analysis of the BOLD fMRI data was performed in this chapter in order to determine the degree of voxel dependence introduced in our acquisition method. This autocorrelation estimate was then used to simulate additive noise on physiologically reasonable hemodynamic response functions. These simulations are important for the performance assessment of the analysis methods developed throughout the thesis. 


\section{Chapter 5}

\section{Analysis Methods: Part I Functional Image Pattern Recognition}

\subsection{Introduction}

The next three chapters put forward a strategy for analysing BOLD fMRI data based on the neurovascular properties discussed in chapter 2 , and on the scanner and physiological effects that were demonstrated in chapter 3. This chapter develops and applies an established unsupervised pattern recognition algorithm called "fuzzy $k$-means," first described by Dunn (1973), to partition the neuroimage data from one session into $k$ clusters of voxel time sequences that are optimally separated according to a least squares objective function. It will be made clear in the discussion section of this chapter why this particular algorithm is suitable for functional neuroimage data analysis. The theoretical material presented in sections 5.2 and 5.3 is the consolidation of my studies synthesising the original work by Dunn (1973) and Bezdek (1981). I have taken their theories and applied them for the pattern recognition of our functional neuroimage data. In section 5.4 , I discuss the idea of partition entropy described by Bezdek (1981) in a probabilistic setting and propose a novel approach to estimate this quantity using null and simulated neuroimage data. In section 5.5, I describe the algorithm implementations used throughout this thesis: a com- 
bination of software I wrote independently and software written by Jarmasz \& Somorjai (2002), which I modified for this research.

Pattern recognition is especially relevant in BOLD fMRI, since the spatial extent of functional hyperemia is on the order of millimetres and lasting several seconds (Malonek \& Grinvald, 1996); therefore, it can be sufficiently sampled in space and time. Secondly, functional hyperemia is known to vary considerably in healthy, and more so, in diseased populations (D'Esposito et al., 2003). Since distinct regions from larger functional circuits interact (sections 1.2 and 2.2), multiple responding regions should be expected and distinguished. Recently for example, Zhou \& Rajapakse (2008) have applied the fuzzy $k$-means algorithm to fMRI data to detect and distinguish between different response signals. However, this algorithm is heuristic in nature, and despite much ongoing effort, it has not yet been directly linked to a probabilistic framework (Bezdek, 1981; Bezdek \& Pal, 1992; Bouguessa et al., 2006; Hathaway et al., 2006). For this reason, in the following two chapters, the heuristically obtained clusters will be modelled parametrically and then submitted to a Bayesian statistical model to determine each cluster's significance.

\subsection{Fuzzy set theory and partitions}

The following definition is applicable in the context of identifying signals within large data sets like those in functional neuroimaging.

Definition 1 (Bezdek (1981)). Pattern recognition is a search for structure in the data.

Here, the data are represented by one neuroimaging session $w[n]$, where we are particularly interested in each voxel time sequence. Thus, we consider our data as being a set of time sequences $w[t]$ indexed by voxel $x \in X$. The structure we seek should explain the relationship between the time sequences of a voxel $x$ and those of other voxels, such that $X$ may be partitioned into subsets of voxels that are related to each other. Hence, the search is the analytical method for obtaining this partition. For now, we will simply associate 
each sequence $w[t]$ with a data point $x$ in an abstract space $X$.

The fuzzy $k$-means algorithm will partition the data indexed by $X$ into $k$ "fuzzy sets," which are a generalisation of the ordinary mathematical concept of a set. Here, the notion of membership imprecision is directly integrated into this fuzzy set theory developed by Zadeh (1965). For example, if we partition $X$ into $k$ subsets $X_{1}, \cdots, X_{k}$, then in the ordinary sense, each $x \in X$ must belong to exactly one subset $X_{i}$. The fuzzy partition, on the other hand, generalises the concept of membership such that $x$ can belong to multiple fuzzy sets with a varying degree of membership. Therefore, the ordinary partition becomes a special case of the fuzzy partition.

Establishing a one-to-one correspondence between a set and a corresponding membership function is the simplest way to rigourously derive the fuzzy set and its generalised subset and equality relations, as well as its intersection, union, and complement operators. Furthermore, this approach will also prove that fuzzy set theory is consistent in the sense that the power set $\mathcal{P}(X)$ (i.e., the class of all fuzzy subsets of $X$ ) is closed under the above mentioned operations. We begin with ordinary sets where the cardinality $|X|$ is always considered to be finite.

Definition 2. Let $X_{i} \subseteq X$. Then the membership function of $X_{i}$ is the mapping $u_{i}: X \longrightarrow\{0,1\}$ defined for all $x \in X$ by the rule

$$
u_{i}(x)= \begin{cases}1, & x \in X_{i} \\ 0, & \text { otherwise }\end{cases}
$$

These membership functions form a one-to-one correspondence between $\mathcal{P}(X)$ and the class of all membership functions because for any $A \in \mathcal{P}(X)$ there is always a unique function $u_{A}$ and vice versa. To see this, let $\mathcal{P}(F)$ denote the class of all membership functions for subsets of $X$. Then, note that the set operations on $A, B \in \mathcal{P}(X)$ are equivalent to the usual relations and operations on real functions $u_{A}, u_{B} \in \mathcal{P}(F)$ for all 
$x \in X$.

$$
\begin{aligned}
A \subseteq B & \Leftrightarrow u_{A}(x) \leq u_{B}(x) \\
A=B & \Leftrightarrow u_{A}(x)=u_{B}(x) \\
\bar{A}=\{x \in X \mid x \notin A\} & \Leftrightarrow \bar{u}_{A}(x)=1-u_{A}(x) \\
A \cap B & \Leftrightarrow \min \left\{u_{A}(x), u_{B}(x)\right\} \\
A \cup B & \Leftrightarrow \max \left\{u_{A}(x), u_{B}(x)\right\}
\end{aligned}
$$

Also, the empty set $\emptyset$ is equivalent to the zero function $0: X \longrightarrow 0$, and the universe set $X$ is equivalent to the one function $1: X \longrightarrow 1$, for all $x \in X$. Therefore, $\mathcal{P}(X)$ and $\mathcal{P}(F)$ along with their respective relations and operations are said to be isomorphic algebras

$$
\left(\mathcal{P}(X), \subseteq,={ }^{-}, \cap, \cup\right) \leftrightarrow\left(\mathcal{P}(F), \leq,={ }^{-}, \min , \max \right)
$$

and can be used interchangeably.

The class of all partitions (of all sizes $k=1, \ldots,|X|$ ) of $X$ form a proper subset of $\mathcal{P}(X)$. Then, more specifically let $\Upsilon(k, X)$ be the class of all $k$-partitions on $X$, where every partition $P \in \Upsilon(k, X)$ has the form $P=\left\{X_{1}, \cdots, X_{k}\right\} \subset \mathcal{P}(X)$ whose elements are of special importance in representing the structure of $X$.

Definition 3. Let $P \in \Upsilon(k, X)$ be a $k$-partition for a data set $X$ and an integer $0<k \leq$ $|X|$. Then, the elements $X_{i} \in P$ are called clusters and have the following properties for all $i=1, \ldots, k$

$$
\begin{gathered}
X_{i} \neq \emptyset, \\
X_{i} \cap X_{j}=\emptyset, \quad i \neq j, \\
\bigcup_{i=1}^{k} X_{i}=X .
\end{gathered}
$$

Equivalently, $P$ can also be defined by the set of membership functions $\left\{u_{1}, \cdots, u_{k}\right\} \in$ $\mathcal{P}(F)$ satisfying the conditions

(a) for all $i=1, \ldots, k$, there exists some $x \in X$ such that $u_{i}(x) \neq 0$, and 
(b) for all $x \in X, \sum_{i=1}^{k} u_{i}(x)=1$.

Therefore, $\Upsilon(k, X)$ can also be written as the $k$-variate membership mapping

$$
\Upsilon(k, X)=\left\{\left\{u_{1}, \ldots, u_{k}\right\}: X \longrightarrow\{0,1\}^{k} \mid u_{i} \text { satisfies (a) and (b) for } i=1, \ldots, k\right\}
$$

This functional representation of $\Upsilon(k, X)$ is the best way to generalise ordinary partitions. Now, a fuzzy subset of $X$ can be formally defined by a simple modification of the membership function in definition 2 .

Definition 4. $A$ fuzzy subset $X_{i}$ of $X$ is defined by the membership function $u_{i}: X \longrightarrow$ $[0,1]$, which maps each $x \in X$ to a real number $y_{i}$ in the unit interval by the rule

$$
u_{i}(x)= \begin{cases}1, & \text { if } x \text { entirely belongs to } X_{i} \\ 0<y_{i}<1 & \text { if } x \text { partially belongs to } X_{i} \\ 0, & \text { otherwise. }\end{cases}
$$

Unlike definition 2, this function has a continuous range on the unit interval and, hence, can describe points having only partial membership to the fuzzy set. The set of all fuzzy membership functions on $X$ from definition 4, can be written as

$$
\Upsilon_{f}(k, X)=\left\{\left\{u_{1}, \ldots, u_{k}\right\}: X \longrightarrow Y_{f} \subset[0,1]^{k}\right\}
$$

where $Y_{f}$ is the diagonal plane cutting through the hypercube $[0,1]^{k}$ given by

$$
Y_{f}=\left\{y \in[0,1]^{k} \mid 0 \leq y_{i} \leq 1, \text { for all } i=1, \ldots, k ; \sum_{i=1}^{k} y_{i}=1\right\}
$$

There are two important observations to make on $\Upsilon_{f}(k, X)$. First, $\Upsilon_{f}(k, X) \supset \Upsilon(k, X)$. This follows immediately from the fact that $[0,1] \supset\{0,1\}$. Consequently, each ordinary partition is embedded in the class of fuzzy partitions. Second, $\Upsilon_{f}(k, X)$ contains degenerate partitions, since condition (a) is not applied in equations (5.13) and (5.14). For example, partitions may contain empty clusters, i.e., with membership function $u_{i}=\mathbf{0}$, exist in $\Upsilon_{f}(k, X)$. Therefore, degenerate $k$-partitions can replicate $(k-1)$-partitions, and 
in general $\Upsilon_{f}(k, X) \supset \cdots \supset \Upsilon_{f}(1, X)$. However, this slightly more general definition will prove convenient when deriving the fuzzy $k$-means algorithm in the next section.

Having described the class of partitions available to analyse $X$, how do we identify the partition that best represents the structure of $X$ ? To answer this optimisation question, we require the notion of distance between data points $x, y \in X$. Hence, the data indexed by $X$ must belong to a metric space $(\mathbb{V}, d)$ where $X \subset \mathbb{V}$, such that we can measure the distance between data points by some metric $d(x, y) \in \mathbb{R}$. Now with a measure of distance, we can form clusters with data points that are closer to each other than to any other point outside the cluster. Moreover, we can also require that the clusters are as far apart from each other as possible. Formally, ordinary partitions $P \in \Upsilon(k, X)$ possessing the property described here are called compact well-separated (CWS) and are quantified according to Dunn (1973) by the ratio of the smallest intercluster distance and the largest intracluster distance

$$
\beta(k, P)=\frac{\min _{1 \leq q \leq k} \min _{\substack{1 \leq r \leq k \\ r \neq k}} \operatorname{dist}\left(X_{q}, \operatorname{conv} X_{r}\right)}{\max _{1 \leq p \leq k} \operatorname{diam}\left(X_{p}\right)},
$$

where $\operatorname{dist}(A, B)=\inf _{x \in A, y \in B} d(x, y), \operatorname{diam}(A)=\sup _{x, y \in A} d(x, y)$, and $\operatorname{conv} A$ is the convex hull of points containing $A$. Finding $\bar{\beta}(k)=\max _{P \in \Upsilon(k, X)} \beta(k, P)$ will quantify the separability of the data set $X$ relative to $d$. Furthermore, Dunn (1973) showed that $(X, d)$ has a CWS $k$-partition if and only if $\bar{\beta}(k)>1$, and the partition $P^{\prime}$ with $\beta\left(k, P^{\prime}\right)=\bar{\beta}(k)$ is optimal.

Having defined structure in $X$ as the optimal $k$-partition, the final question is how do we search for it? Even for small data sets, the partition class $\Upsilon(k, X)$ is immense, making exhaustive search practically impossible. For example, Duda \& Hart (1972) showed that for $|X|=n$ and some integer $1 \leq k \leq n$, the cardinality of $\Upsilon(k, X)$ is exactly

$$
|\Upsilon(k, X)|=\frac{1}{k !} \sum_{i=1}^{k}\left(\begin{array}{c}
k \\
i
\end{array}\right)(-1)^{k-i} i^{n}
$$


Hence, consider this problem for a small data set of size $|X|=32$. In addition, suppose it is known a priori that there are $k=3$ underlying clusters in $X$. Then equation (5.16) indicates that there are over 308 trillion possible 3-partitions in $\Upsilon(3, X)$. A futuristic supercomputer capable of calculating $\beta(3, P)$ per nanosecond given $P$, would need about 3.5 days to scan all of $\Upsilon(3, X)$. The amount of time required grows factorially as $|X|$ grows as well as when $k$ grows from 1 to $|X| / 2$. For example, to scan instead $\Upsilon(4, X)$ on the same computer would require about 24 years!

\subsection{Fuzzy $k$-means on time sequence data}

Heuristic algorithms searching for partitions in $\Upsilon(k, X)$ that maximise $\beta(k, P)$ have been studied. In particular, Ball \& Hall (1967) developed the (ordinary) $k$-means, or ISODATA, algorithm that will find the optimal $P^{\prime} \in \Upsilon(k, X)$ provided $\bar{\beta}(k) \gg 1$ is known a priori. This will not do for our problem for two key reasons. First, we do not know a priori the value of $\bar{\beta}(k)$ for any $k$. Consequently, we do not know what value of $k$ to use. Second, given $k$, this algorithm will always converge to some partition in $\Upsilon(k, X)$ regardless of whether the data are CWS or not.

The fuzzy $k$-means algorithm bypasses these limitations and provides additional advantages. By searching instead within the fuzzy partition class $\Upsilon_{f}(k, X) \supset \Upsilon(k, X)$, the algorithm can still converge to the optimal ordinary partitions for CWS data and, in addition, converge to fuzzy partitions when the data are in fact non-CWS. Secondly, by extending this optimisation problem into a smooth space (requiring that the membership functions be smooth), the algorithm can be derived using calculus. Moreover, for each cluster, the optimal function $u_{i}$ quantifies the imprecision of each $x \in X_{i}$. Therefore, we can obtain the degree of structure in the data by only examining the set of membership functions. Finally, we can compare optimal $k$-partitions based on their imprecision for various values of $k$. Given the set of membership functions $U=\left\{u_{1}, \cdots, u_{k}\right\}$, we can 
compute the corresponding set of average data points called centroids $V=\left\{v_{1}, \cdots, v_{k}\right\}$, where $v_{i}=\left\langle u_{i}(x) x\right\rangle \in \mathbb{V}$ for $i=1, \ldots, k$.

The following theorem is the central result validating the algorithm's convergence to the optimal partition. I have developed a proof based on Dunn (1973) to demonstrate the machinery of the algorithm and to explicitly derive the necessary iterative steps for implementation.

Theorem 2 (Dunn (1973)). If the membership functions $U^{\prime} \in \Upsilon_{f}(k, X) \subset \mathbb{V}$ and centroids $V^{\prime} \in \mathbb{V}$ minimise the least squares distance objective function

$$
J(U, V, X)=\sum_{i=1}^{k} \sum_{x \in X} u_{i}^{2}(x) d^{2}\left(x, v_{i}\right),
$$

then they satisfy the three following conditions for all $i=1, \ldots, k$.

1. For each fixed $x \in X$, let $I=\left\{1 \leq i \leq k \mid v_{i}^{\prime}=x\right\}$ be the index set of clusters where $x$ is an exclusive member. Let $\bar{I}=\left\{1 \leq i \leq k \mid v_{i}^{\prime} \neq x\right\}$ be the complement set of $I$. Then, there are two possible cases.

$A: I=\emptyset$. Then, $x$ has membership values given by

$$
u_{i}^{\prime}(x)=\frac{1 / d^{2}\left(x, v_{i}^{\prime}\right)}{\sum_{j=1}^{k} 1 / d^{2}\left(x, v_{j}^{\prime}\right)} .
$$

$B: I \neq \emptyset$. Then, some $j \in \bar{I}$ implies that $u_{j}^{\prime}(x)=0$, and in particular

$$
\sum_{\substack{i \in I \\ i \neq j}} u_{i}^{\prime}(x)=1 .
$$

2. The partition $U^{\prime}$ always consists of non-empty fuzzy sets. That is, there exists an $x \in X$ such that $u_{i}^{\prime}(x) \neq 0$.

3. The clusters satisfy

$$
v_{i}^{\prime}=\frac{\sum_{x \in X} u_{i}^{\prime 2}(x) x}{\sum_{x \in X} u_{i}^{\prime 2}(x)} .
$$

Proof. It is sufficient to show that each condition must be true if $U^{\prime}$ and $V^{\prime}$ solve equation (5.17). 
Condition 1: The solutions $U^{\prime}$ and $V^{\prime}$ must satisfy

$$
\sum_{i=1}^{k} \sum_{x \in X} u_{i}^{\prime 2}(x) d^{2}\left(x, v_{i}^{\prime}\right) \leq \sum_{i=1}^{k} \sum_{x \in X} u_{i}^{2}(x) d^{2}\left(x, v_{i}\right)
$$

for all $U \in \Upsilon_{f}(k, X)$ and $V \in \mathbb{V}$. This statement is also true for each $x \in X$ because of the condition $\sum_{i=1}^{k} y_{i}=1$ imposed on the range of the mapping $U: X \longrightarrow Y_{f}$ in equation (5.14). To see this for each $x \in X$, arrange the distances $d\left(x, v_{i}\right)$ over $i$ in increasing order, and correspondingly, set the numbers $y_{i}^{\prime}=u_{i}^{\prime}(x)$ to be monotonically decreasing. Therefore, $U^{\prime}$ and $V^{\prime}$ must also satisfy

$$
\begin{aligned}
\sum_{i=1}^{k} u_{i}^{\prime 2}(x) d^{2}\left(x, v_{i}^{\prime}\right) & \leq \sum_{i=1}^{k} u_{i}^{2}(x) d^{2}\left(x, v_{i}\right) \\
& =\min _{y \in Y_{f}} \sum_{i=1}^{k} y_{i}^{2} d^{2}\left(x, v_{i}\right)
\end{aligned}
$$

for all $x \in X$.

For the case $I=\emptyset, x$ has fuzzy membership in several clusters. The argument $y^{\prime} \in Y_{f}$ that minimises equation (5.17) can be calculated by the method of Lagrange multipliers, since equation (5.17) is quadratic in $U$ with a non-negative minimum, and the range $Y_{f}$ is smooth. Furthermore, the possibility that $y^{\prime}$ is an end point of $Y_{f}$ must be avoided. Hence, consider instead the extended range without bounds

$$
\bar{Y}_{f}=\left\{y \in \mathbb{R}^{k} \mid \sum_{i=1}^{k} y_{i}=1\right\} \supset Y_{f} .
$$

Then, the objective is to minimise the function

$$
f(y)=\sum_{i=1}^{k} y_{i}^{2} d^{2}\left(x, v_{i}^{\prime}\right)
$$

subject to the constraint

$$
g(y)=\sum_{i=1}^{k} y_{i}-1=0, \quad y \in \mathbb{R}^{k} .
$$

According to Lagrange's method, since the gradient $\nabla g(y) \neq 0$ for all $y \in \mathbb{R}^{k}$, then there exists a number $\lambda^{\prime} \in \mathbb{R}$ such that the ordered pair $\left(y^{\prime}, \lambda^{\prime}\right)$ is a critical point of the Lagrangian function

$$
L(y, \lambda)=f(y)+\lambda g(y)
$$


Thus, we calculate the partial derivatives for each $i=1, \ldots, k$

$$
\begin{aligned}
& \frac{\partial L}{\partial \lambda}=y_{i}-1=0 \\
& \frac{\partial L}{\partial y_{i}}=2 y_{i} d^{2}\left(x, v_{i}^{\prime}\right)+\lambda=0
\end{aligned}
$$

and by substitution, the solutions are obtained

$$
\begin{aligned}
\lambda^{\prime} & =\frac{-1}{\sum_{j=1}^{k} 1 / 2 d^{2}\left(x, v_{j}^{\prime}\right)} \\
y_{i}^{\prime} & =-\lambda^{\prime} / 2 d^{2}\left(x, v_{i}^{\prime}\right) \\
& =\frac{1 / d^{2}\left(x, v_{i}^{\prime}\right)}{\sum_{j=1}^{k} 1 / d^{2}\left(x, v_{j}^{\prime}\right)} .
\end{aligned}
$$

From this last equation for $y_{i}^{\prime}$, note that $I=\emptyset$ implies that $0<y_{i}^{\prime}<1$ for all $i=1, \ldots, k$. Therefore, we are guaranteed that the solutions $y^{\prime}$ lie in $Y_{f}$ and not on the extension $\bar{Y}_{f}-Y_{f}$. Moreover, they are unique solutions of the membership functions $u_{i}^{\prime}(x)=y_{i}^{\prime}$ for all $x \in X$.

For the case $I \neq \emptyset, x$ belongs exclusively to some cluster $X_{j}, j \in I$, and it must be that $u_{i}^{\prime}(x)=0$ for all $i \in \bar{I}$. Consequently, the minimum attained in equation (5.17) must be zero. Otherwise, if for some $i \in \bar{I}$ we have $y_{i} \neq 0$, then a contradiction arises because $u_{i}^{\prime 2}(x) d^{2}\left(x, v_{i}^{\prime}\right)>0$ is sub-optimal. Therefore, condition 1 must be true if $U^{\prime}$ satisfies equation (5.21), that is, $u_{i}^{\prime}(x)=0$ for all $i \in \bar{I}$.

Condition 2: Suppose, on the contrary, that this condition is false, such that for some cluster $j$ we have $u_{j}^{\prime}(x)=0$ for all $x \in X$. Then in addition to $V^{\prime}$ a distinct set $V^{\prime \prime}$ also satisfies (5.21), where the $j^{\text {th }}$ centroid is forced to equal an unclaimed point $\hat{x} \in X$ outside of $X_{j}$. Such a point must always exist if this condition is false because $k \leq|X|$ and $u_{j}^{\prime}(x)=0$ for all $x \in X$ imply that $X_{j}=\emptyset$. But this contradicts the premise that condition 2 is false, since by condition 1 it must be that $v_{j}^{\prime \prime}=\hat{x}$ if and only if $u_{j}^{\prime}(\hat{x})=1$. Therefore, condition 2 must also hold if $U^{\prime}$ and $V^{\prime}$ satisfy the theorem.

Condition 3: To verify the final condition, which gives the form of the centroid functions, we calculate the directional derivative of the objective function at its minimum, which must be flat in all directions, and solve for $V^{\prime}$. Consider the objective function for $V$ with $U$ fixed $f(V)=J(U, V, X)$. Then, if $U^{\prime}$ and $V^{\prime}$ satisfy equation (5.21) the directional derivative $D_{Q} f(V)$ must also vanish for all vectors $Q \in \mathbb{V}$, provided that the metric is inner-product induced, that is, of the form $d(x, y)=\sqrt{(x-y) \cdot(x-y)}$. To see this, the 
fixed objective can be written as

$$
\begin{aligned}
f\left(V^{\prime}\right) & =\sum_{i=1}^{k} \sum_{x \in X} u_{i}^{\prime 2}(x) d^{2}\left(x, v_{i}^{\prime}\right) \\
& =\sum_{i=1}^{k} \sum_{x \in X} u_{i}^{\prime 2}(x)\left(\left(x-v_{i}^{\prime}\right) \cdot\left(x-v_{i}^{\prime}\right)\right) .
\end{aligned}
$$

Evaluating its directional derivative at $V^{\prime}$ yields, by definition,

$$
D_{Q} f\left(V^{\prime}\right)=\left.\frac{d}{d h} f\left(V^{\prime}+h Q\right)\right|_{h=0} .
$$

Let $\xi=x-v_{i}-h q_{i}$ be the $i^{\text {th }}$ component of $V^{\prime}+h Q$. Then, by the chain rule we get

$$
\begin{aligned}
\left.\frac{d}{d h} f\left(V^{\prime}+h Q\right)\right|_{h=0} & =\left.\frac{d f}{d \xi} \frac{d \xi}{d h}\right|_{h=0} \\
& =\left.\left(\frac{d}{d \xi} \sum_{i=1}^{k} \sum_{x \in X} u_{i}^{\prime 2}(x)(\xi \cdot \xi)\right)\left(\frac{d}{d h}\left(x-v_{i}^{\prime}-h q_{i}\right)\right)\right|_{h=0} \\
& =\left.\left(2 \sum_{i=1}^{k} \sum_{x \in X} u_{i}^{\prime 2}(x)(\xi \cdot 1)\right)\left(-q_{i}\right)\right|_{h=0} \\
& =-2 \sum_{i=1}^{k} \sum_{x \in X} u_{i}^{\prime 2}(x)\left(\left(x-v_{i}^{\prime}\right) \cdot q_{i}\right)
\end{aligned}
$$

This equation must vanish for all $Q \in \mathbb{V}$. Hence, from the last line we have

$$
\sum_{i=1}^{k} \sum_{x \in X} u_{i}^{\prime 2}(x)\left(\left(x-v_{i}^{\prime}\right) \cdot q_{i}\right)=0
$$

which can only vanish over the outer sum if each component is zero. That is, it must be that

$$
\sum_{x \in X} u_{i}^{\prime 2}(x)\left(\left(x-v_{i}^{\prime}\right) \cdot q_{i}\right)=0
$$

for all $Q \in \mathbb{V}$. Continuing in this reductive fashion, we obtain

$$
\begin{aligned}
\sum_{x \in X} u_{i}^{\prime 2}(x)\left(x-v_{i}^{\prime}\right) & =0 \text { for all } i=1, \ldots, k \\
\Rightarrow \sum_{x \in X} u_{i}^{\prime 2}(x) v_{i}^{\prime} & =\sum_{x \in X} u_{i}^{\prime 2}(x) x .
\end{aligned}
$$

Solving for $v_{i}^{\prime}$ in this last equation shows that condition 3 must also be true, therefore, completing the proof. 
This theorem and its proof show explicitly how to obtain from a data set $X \subset \mathbb{V}$ its $k$-partition of maximally compacted clusters that are maximally separated from each other. Specifically, the solution that minimises the objective $J(U, V, X)$ also maximises $\bar{\beta}(k)$, hence produces clusters that are CWS or as close to it as possible. The theorem gives the form of the optimal functions for $U$ and $V$ in equations (5.18) and (5.20), respectively. The proof indicates the mathematical requirements that guarantee the solution exists and is unique, namely, that the membership functions $U$ be smooth and that the metric $d$ be inner-product induced from the space $\mathbb{V}$. From these results, the algorithm to find the CWS optimal solution is as follows.

Algorithm 2 (Fuzzy $k$-means (Dunn, 1973)). Given a finite data set $X$ within a metric space $(\mathbb{V}, d)$ of dimension $D$, an integer $1 \leq k \leq|X|$, and a real number $\epsilon>0$, do the following to obtain a CWS optimal $k$-partition of $X$.

1. Choose an initial non-degenerate partition $U_{0} \in \Upsilon_{f}(k, X)$.

2. Compute the initial centroids $V_{0} \in \mathbb{V}$ according to

$$
v_{i}=\frac{\sum_{x \in X} u_{i}^{2}(x) x}{\sum_{x \in X} u_{i}^{2}(x)}, \quad \text { for } i=1, \ldots, k .
$$

3. Compute a new partition $U \in \Upsilon_{f}(k, X)$ according to the rule: for each $x \in X$, let $I=\left\{i \mid v_{i}=x\right\}$. If $I=\emptyset$, then

$$
u_{i}(x)=\frac{1 / d^{2}\left(x, v_{i}\right)}{\sum_{j=1}^{k} 1 / d^{2}\left(x, v_{j}\right)}, \quad \text { for } i=1, \ldots, k .
$$

If $I \neq \emptyset$, let $j=\min (I)$ and for each $i=1, \ldots, k$

$$
u_{i}(x)= \begin{cases}1 & i=j \\ 0 & \text { otherwise }\end{cases}
$$

4. Compute the new centroids $V$ using $U$ as in step 2, and compute the maximum norm defect

$$
\delta=\max _{1 \leq i \leq k} \max _{1 \leq j \leq D}\left|v_{0 i, j}-v_{i, j}\right|
$$

where $v_{i, j}$ is the $j^{\text {th }}$ component of vector $v_{i}$ relative an arbitrary basis for $\mathbb{V}$. 
5. If $\delta<\epsilon$, then stop. Otherwise, let $U_{0}=U, V_{0}=V$, and repeat the algorithm starting from step 3 .

Now equipped with the machinery to search for structure in our data, we return to the applied problem. Our data set $X$ is the set of voxel time sequences $w[t]$ acquired in one neuroimaging session. Therefore, each voxel $x$ can be uniquely put into correspondence with a vector in $\mathbb{V}$ whose components are given by $w[x, t]$ for $t=0, \ldots, T-1$. Therefore, it is reasonable to choose the Euclidean space $\mathbb{V}=\mathbb{R}^{T}$ to represent our data. The structure we seek must describe the temporal relationship between different voxels by producing voxel clusters whose members are maximally correlated. While the obvious choice for a metric is the Euclidean distance $d(x, y)=\|x-y\|_{2}=\sqrt{\sum_{t=0}^{T-1}(w[x, t]-w[y, t])^{2}}$, we choose instead a metric that is sensitive to signal phase and invariant to signal amplitude so that we may obtain clusters whose voxels are correlated regardless of their amplitudes. Golay et al. (1998) proposed a pseudo-metric exactly for this purpose called the hyperbolic correlation distance, which they show is superior to Euclidean distance and to Pearson's correlation coefficient for clustering BOLD fMRI data. Their pseudo-metric is based on Pearson's correlation

$$
r(x, y)=\frac{1}{(T-1) \hat{\sigma}[x] \hat{\sigma}[y]} \sum_{t=0}^{T-1}(w[x, t]-\hat{\eta}[x])(w[y, t]-\hat{\eta}[y])
$$

such that the value $r(x, y)$ is uniquely mapped onto the non-negative real numbers according to the hyperbolic distance

$$
d(x, y)=\sqrt{\frac{1-r(x, y)}{1+r(x, y)}}, \quad \text { where }-1<r(x, y) \leq 1
$$

This function has several interesting properties. First, it is not inner-product induced and, therefore, the validity of theorem 2 is not necessarily guaranteed. However, Golay et al. (1998) prove that the fuzzy $k$-means algorithm does in fact converge properly using the hyperbolic correlation distance. Second, $d(x, y)$ is directly proportional to the angle 
$\theta$ between of the mean-centred vectors $w[x, t]-\hat{\eta}[x]$ and $w[y, t]-\hat{\eta}[y]$, since the cosine rule $(x \cdot y)=2\|x\|\|y\| \cos (\theta)$ leads immediately to the fact that $r(x, y)=\cos (\theta) /(T-1)$. Finally, Golay et al. (1998) show that if the random process $\mathbf{w}_{t}$ is i.i.d., then $d(x, y)$ is equal to the $Z$-score that tests if the samples $x$ and $y$ are statistically different. Of course, we know from chapter 4 that $\mathbf{w}_{t}$ is not i.i.d. and, therefore, do not make statistical inference at this stage.

\subsection{Partition entropy minimisation}

As mentioned in the previous section, the solution $U$ is all that is necessary to examine the degree of structure present in $X$. Consider, for example, a uniformly distributed data set. Then for any value of $k$, the solution $U$ always consists of $k$ identical constantvalued functions of the form $u_{i}(x)=1 / k$. Hence, a point $x$ belongs to all clusters equally and $U$ does not yield novel information. On the other hand, suppose $X$ is CWS and bi-modally distributed. Then the choice of $k$ will influence $U$ considerably. If $k=2$, then the solution $U$ will converge to two Heaviside membership functions, each centred on one mode and taking on values 0 or 1 depending on $x$. Here, there is a clear distinction on the membership, and $U$ is unambiguous to which cluster each point $x$ belongs. In Shannon's notion of information entropy, the latter example has low entropy while the former has high entropy. Suppose in the latter example we erroneously choose $k=3$, then $U$ is forced to split the two modes into three clusters, invariably introducing imprecision in the membership of some points. Bezdek (1981) applies Shannon's measure to $U$ in order to choose the number $k$ that leads to the most informative partition by minimising its entropy. It is reasonable to apply information entropy to $U$ because this system of functions has the same properties of a set of probability mass functions. In particular, for all $x \in X$, we have $\sum_{i=1}^{k} u_{i}(x)=1$ and $u_{i}(x) \geq 0$.

Definition 5. The partition entropy of a given partition $U \in \Upsilon_{f}(k, X)$ in bits of infor- 


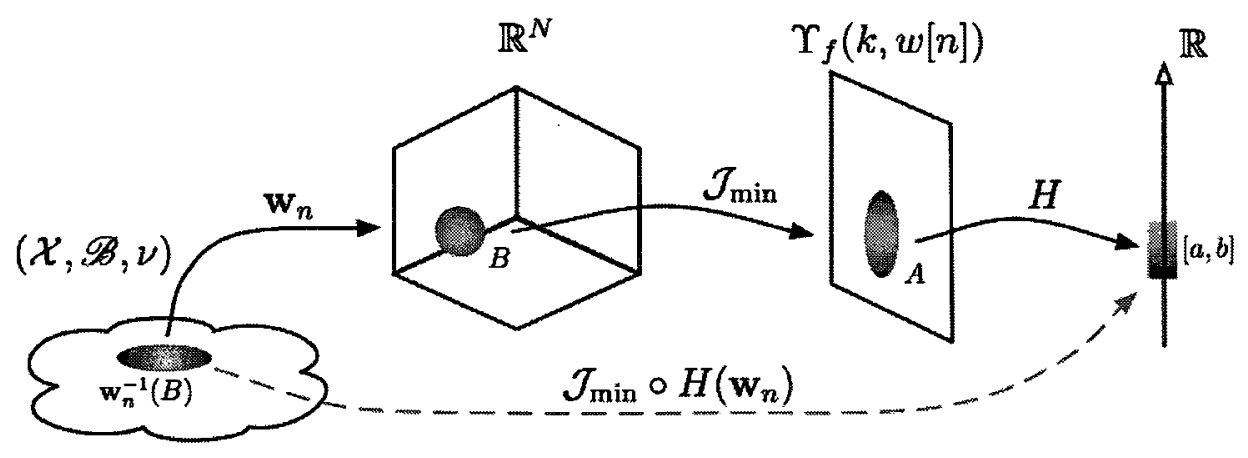

Figure 5.1: Relation between data and their partition entropy. Obtaining a probability distribution of $H$ requires knowledge of the composition of functions $\mathcal{J}_{\min } \circ H\left(\mathbf{w}_{n}\right)$.

mation is given by

$$
H(U)=-\frac{1}{|X|} \sum_{x \in X} \sum_{i=1}^{k} u_{i}(x) \log _{2}\left(u_{i}(x)\right),
$$

where $u_{i}(x) \log _{2}\left(u_{i}(x)\right)=0$ for $u_{i}(x)=0$.

Interpreting this definition as a statistic leads to the following question that remains an open problem in the literature: what is the probability distribution of $H$ given a random process $\mathbf{w}_{n}$ ? Knowing the distribution of $H$ allows the formulation of hypothesis-driven tests to distinguish structured processes $(k>1)$ from uniform ones $(k=1)$. Unfortunately, calculating the distribution of $H$ even with a complete probabilistic description of $\mathbf{w}_{n}$ is still difficult because the fuzzy $k$-means algorithm does not have a closed analytic form. Consequently, the mapping from $w[n] \in \mathbb{R}^{N}$ to $U \in \Upsilon_{f}$ is not accessible. Figure 5.1 illustrates how the chain of transformations beginning from the probability space underlying the random process $\mathbf{w}_{n}$, through the clustering algorithm, represented by the symbol $\mathcal{J}_{\text {min }}$, and the partition space $\Upsilon_{f}$ lead to the entropy measure $H$ taking values on the real line. The statistical question we seek will give us the probability of acquiring data $w[n]$ with a partition entropy that falls in an arbitrary interval $[a, b] \in \mathbb{R}$. By definition, the random process $\mathbf{w}_{n}$ is a one-to-one function that maps an event from the probability space $(\mathcal{X}, \mathscr{B}, \nu)$ to a subset $B \subset \mathbb{R}^{N}$, such that the probability of acquiring a data set $w[n] \in B$ is modelled by the measure of its inverse mapping, that is, $\operatorname{Pr}\left\{\mathbf{w}_{n} \in B\right\}=\nu\left(\mathbf{w}_{n}^{-1}(B)\right)$. 
Formally, the probability required is expressed as $\operatorname{Pr}\left\{\mathcal{J}_{\min } \circ H\left(\mathbf{w}_{n}\right) \in[a, b]\right\}$. However, the partition entropy function is many-to-one and, hence, does not have an inverse $H^{-1}$. The fuzzy $k$-means algorithm does not have an analytic form; hence, its inverse $\mathcal{J}_{\min }{ }^{-1}$ is unknown. Nonetheless, $H$ and $\mathcal{J}_{\min }$ are deterministic functions, and so by estimating $\operatorname{Pr}\left\{\mathbf{w}_{n} \in B\right\}$, we can obtain a lower bound for the probability $\operatorname{Pr}\left\{\mathcal{J}_{\min } \circ H\left(\mathbf{w}_{n}\right) \in[a, b]\right\}$. Based on this rationale, it may be possible to estimate the distribution of $H$ under the implementation of fuzzy $k$-means in algorithm 2 using the bootstrap simulation procedure described in algorithm 1 (page 60) of the acquired null fMRI data. That is, one could compute a histogram distribution for $H$ using an ensemble of bootstrap samples $\left\{w_{b}^{(j *)}[t]\right\}$, for $j *=1, \ldots, J *$. From these simulated $H$ values, we could obtain a lower bound estimate of the $95 \%$ confidence interval generated by simulations of uniformly distributed data and bootstrap null fMRI data in $\mathbb{R}^{N}$.

\subsection{Implementation and results}

Two separate implementations of the fuzzy $k$-means algorithm were used in this research. The first implementation was written in Matlab 2009a platform (The MathWorks, Natick, USA) explicitly for this thesis and ongoing research in the current laboratory. While this implementation provides us with direct control over the entire process, it is severely limited in processing speed and data size. For example, for one neuroimaging session as described here, our computational limit is $k=40$ completing analysis in roughly 12 hours on a Microsoft Windows XP Professional operating system (Microsoft, Redmond, USA), running on a Dell XPS workstation (Dell, Round Rock, USA) equipped with a Intel Core 2 Duo $2.66 \mathrm{GHz}$ CPU (Intel, Santa Clara, USA) and $3 \mathrm{~GB}$ of memory. This Matlab implementation serves as testing software to study the algorithm and perform the partition entropy calculations described in this chapter, but it is not suitable for the large scale processing of the numerous neuroimage data sets acquired and simulated 


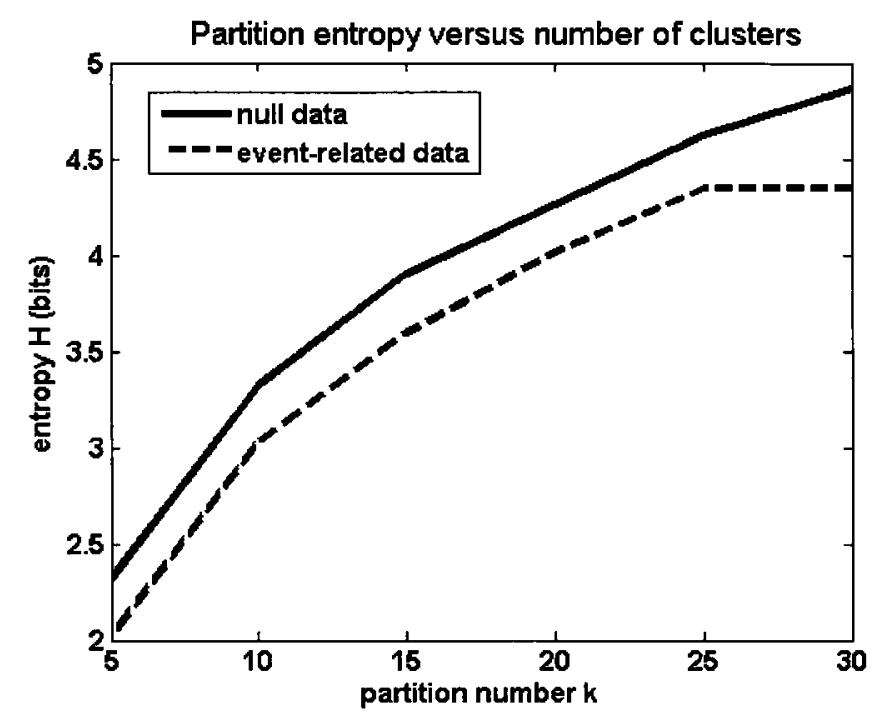

Figure 5.2: Comparison of partition entropy estimates between a null fMRI session and an event-related fMRI session.

during this research. We attempted to perform the bootstrap-based estimate for $H$, but unfortunately the computational resources required was too great for our workstation. Essentially, during the processing a single data set, our machine would run out of memory and unexpectedly quit. Thus, in order to perform a computationally-intensive estimate over hundreds of bootstrap samples on our machine, we would require hundreds of days and an operator to handle the rather unpredictable failures caused by memory consumption. Instead, we were able to produce a partition entropy comparison between real fMRI data sets acquired during a null task and the event-related motor task in a normal subject for $k$ ranging between 5 and 30 in steps of 5 . This is shown in figure 5.2. Although a modest result at best, this single graph took over two days of computation with memory failure handling on our workstation. As a preliminary result, it demonstrates that the active data set has marginally lower entropy for all $k$ tested. As expected in both cases, the entropy gradually declines as $k$ increases, which illustrates the requirement of a normalisation step relative to a uniformly distributed data set, so that the optimal $k$ might be identified.

To address this limitation, a second implementation was used that is based on the $\mathrm{C}++$ source code from the EvIdent software package (National Research Council, Win- 
nipeg, Canada) designed for analysing BOLD fMRI data by Jarmasz \& Somorjai (2002). We modified the code and rebuilt the application using the $\mathrm{C}++$ Microsoft Visual Studio 2005 platform (Microsoft, Redmond, USA) to provide us with binary data files for the cluster membership $U$ and centroid $V$ data. This particular implementation was chosen due to its availability, processing speed, and its cluster merging feature, which heuristically determines the optimal value for $k$ according to the memberships $U$ between each iteration of the algorithm by merging clusters with similar membership functions (Jarmasz \& Somorjai, 1998). Our version of this program runs with parameters set to execute algorithm 2 as shown with a randomly selected initial partition of 40 clusters and tolerance $\epsilon=0.01$. In this implementation, cluster $X_{k}$ is a "rejection cluster" that contains voxels whose time sequences are uncorrelated amongst themselves and all other $k-1$ clusters. This $\mathrm{C}++$ implementation was used to analyse all neuroimage data sets and converged to a stable $k$-partition for each session in a few seconds on the workstation described above. The number of clusters obtained in these data sets was between 16 and 36. Finally, the algorithm converged before reaching a maximum allowed number of 35 iterations in all data sets. Figure 5.3 illustrates the distribution of voxels throughout a $k$-partition obtained with this algorithm from a normal subject performing the event-related paradigm. In this case, 24 clusters are identified, and voxels are rendered in green in the cluster possessing its highest membership value. The surface rendered in grey illustrates the position of the voxels forming the boundary of the brain. The orientation of each image has the subject obliquely facing towards the bottom-left side of the viewer. The eyes are rendered only to clarify the orientation, however, were not included in the analysis. The observations made here are representative of the clustering results throughout all subjects. First, the union of all $k$ clusters form the entire cerebral region analysed. Second, clusters 1 to $k-1$ are sparse and possess a large contiguous region as well as sporadically distributed voxels. Third, many clusters have an interhemispheric symmetry (e.g., $i=21,22,23)$. This is a physiologically plausible phenomenon, given that the cerebral vasculature and macroscopic anatomy (c.f. 

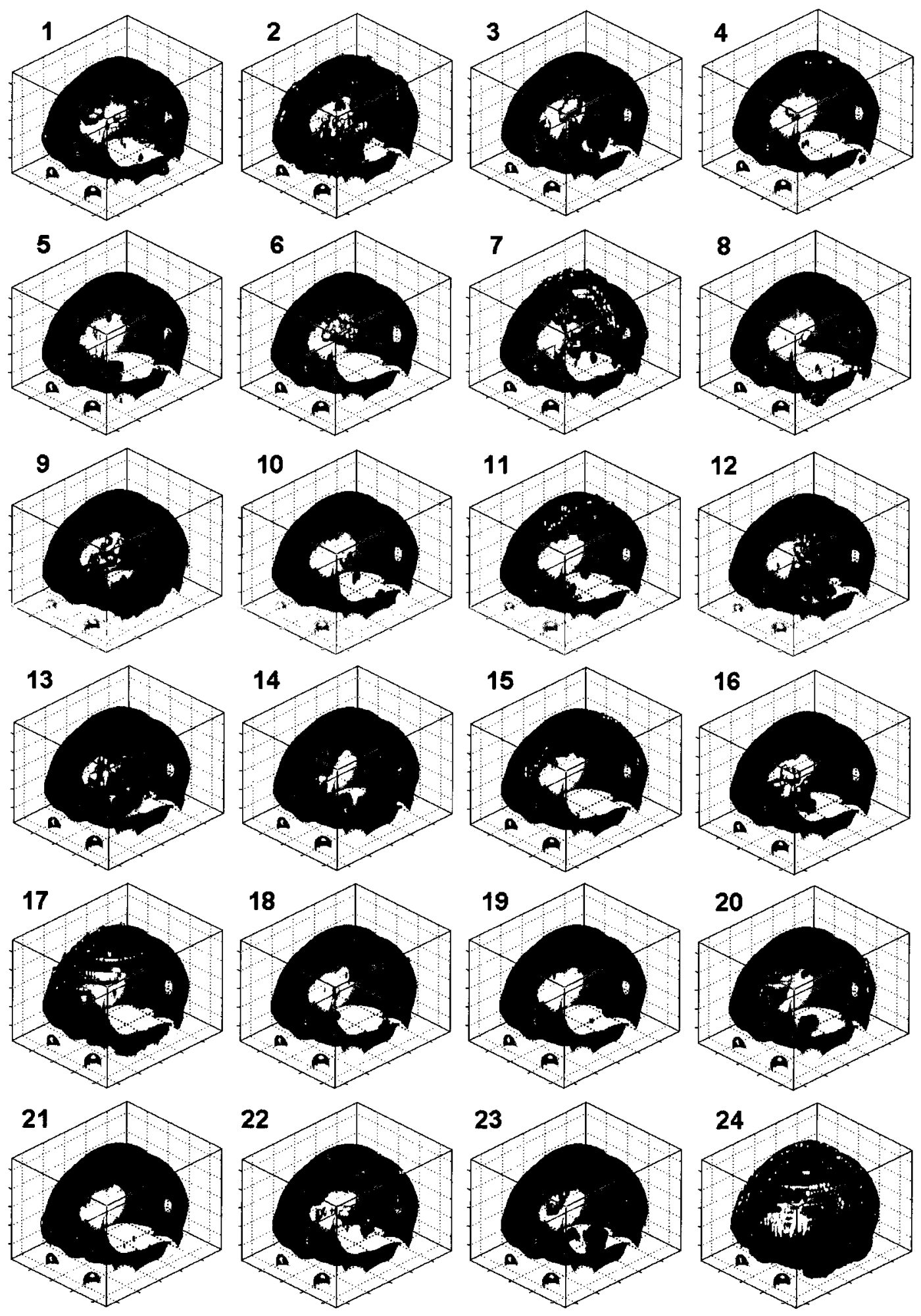

Figure 5.3: Illustration of a $k$-partition obtained from a normal subject performing the event-related visual feedback controlled motor paradigm. Member voxels (green) appear in the cluster with maximum membership. The brain surface is rendered (grey) for intercluster comparison. 
figure 2.4, page 29), as well as the cortical cytoarchitecture (c.f. figure 1.1, page 4, and Garey (1994)) also exhibit interhemispheric symmetry to some extent. Finally, the last cluster $(i=24)$ contains the remainder of voxels with uncorrelated time sequences. This cluster is large and contains the majority of the subcortical cerebral volume. Unlike the other clusters, these voxels do not exhibit prominent MR signal fluctuations beyond the noise level determined by the average signal standard deviation.

\subsection{Discussion}

This chapter describes the theory, application, and implementation of the pattern recognition algorithm used for functional neuroimage analysis in this research. The reason for selecting fuzzy $k$-means algorithm for this problem is primarily based on its ability to identify and distinguish time sequence patterns in BOLD fMRI data without assuming specific patterns a priori. It accomplishes this by clustering the data into maximally separated clusters with the unique advantage of quantifying how close a data set is to being CWS based on the membership functions. In the case of CWS data, the algorithm produces membership functions identical to those of the ordinary $k$-means, i.e., assuming only 0 or 1 values. Although the algorithm is iterative, Dunn (1973) also shows that it possess the descent property of always moving $U$ closer to the optimal solution $U^{\prime}$ with additional iterations. This distinction between the ordinary and fuzzy variants of $k$-means justifies why the latter type is most suitable. This justification follows from the fact that functional neuroimage data do not form a set of independent measurements but rather highly correlated ones, foremost because they represent the operation of a complex system with many interacting parts. Consequently, it is reasonable, and cautious, to expect an appreciable degree of similarity between clusters instead of expecting these data to be CWS. Therefore, fuzzy $k$-means was chosen because it produces the maximally compact and separated partition where the presence of the CWS property can be known directly 
from the partition membership functions.

The principal caveat of this algorithm is the difficulty in linking the parameters $k$ and $U$ with a probability framework so that $k$ and membership value $u_{i}(x)$ can be based on thresholds of statistical significance (Bezdek \& Pal, 1992; Duda et al., 2001). Even if this were possible, the probability density space for the partition space is highdimensional and would likely require heuristics to find the density extremum. A good example of this is a recent Bayesian model for hierarchically clustering time series data from gene expression microarrays (Ramoni et al., 2002). The authors correctly account for temporal autocorrelation with an autoregressive model yet still have to resort to heuristics to find the joint posterior density maximum corresponding to the partition with highest probability of being correct. Since the proposition of the fuzzy $k$-means algorithm in 1973, a Bayesian model for the partition space remains an open problem most likely because of the difficulty in pushing a probability measure forward into the partition and entropy spaces. To address this issue, we attempted to estimate from below the partition entropy versus $\mathrm{k}$ in uniform, null fMRI, and event-related fMRI data sets of equal size. However, we found the computational requirements overwhelming for our workstation and, hence, can only recommend the exploration of this topic for future work. At the very least, the failure to explore this issue caused by a lack of computational power demonstrates the challenges ahead in analytical methods for large and complex data sets.

A practical disadvantage of this algorithm is the difficulty in applying it to on-line problems, since it requires the entire data set $X$ and, consequently, the computational requirements are usually quite high for realistic data sets. A final potential issue revolves around the hyperbolic correlation distance as the pseudo-metric for the algorithm, which is invariant to signal amplitude. Although this distance is shown by Golay et al. (1998) to be more suitable for fMRI data than the Euclidean and standard correlation distances, it is possible that the relevant physiological or pathological states may alter only the magnitude of the measured signal and would therefore go undetected. Evidence of such a 
state would require the use of complimentary metrics or preprocessing techniques.

In conclusion, the obtained clusters from this heuristic exploratory method are useful in identifying and distinguishing patterns of correlated time sequences within BOLD fMRI data without providing pattern templates a priori. This is especially important in stroke, where pathological changes in functional hyperemia are known to occur. In the next two chapters, the space-time structure of these clusters are parameterised and a Bayesian statistical model is applied in order to determine which clusters are responding to the event-related paradigm. 


\section{Chapter 6}

\section{Analysis Methods: Part II Space-Time Structure of the BOLD Signal}

\subsection{Introduction}

The previous chapter used an exploratory pattern recognition method to partition the BOLD fMRI data acquired during one session into clusters having correlated time sequences. Due to the inherent physiological variability in these data, this partition was produced without preconception of the spatial and temporal structure of the clusters. Consequently, the obtained clusters are not necessarily all due to the stimulus response signal of interest but may also represent other underlying processes. Therefore, a set of cluster selection criteria is necessary to objectively determine the salience of each cluster to the experimental paradigm.

To address this requirement, a novel method is proposed for the automated interpretation of clusters identified by exploratory pattern recognition techniques, such as those identified by fuzzy $k$-means discussed in the previous chapter. The selection criteria are formulated using 1) the spatial contiguity of the each voxel cluster, and 2) the temporal cross-correlation between the cluster time sequence and the motor response to an event-related paradigm. The spatial contiguity and temporal cross-correlation para- 
metrically model the space-time structure of each cluster, and the selection criteria are based on the determination of thresholds for each parameter value. The proposed method is demonstrated using BOLD fMRI data acquired from normal subjects performing the event-related visual feedback controlled motor paradigm described in chapter 3 . To verify that the clusters are consistent with the expected normal BOLD response signal (c.f. signal 1 in figure 4.3a, page 57), a comparison is made with the activation maps generated from the established model-based GLM- $t$ test available in the SPM software package (Friston et al., 1995).

Based on the expected sensorimotor system behaviour as well as on the observed $t$-map results testing for the normal BOLD response, the results shown here suggest that these criteria are suitable to distinguish responding clusters appearing in sensorimotorrelated anatomical areas from other clusters with no apparent relation to the paradigm. Moreover, the identified clusters each identify anatomically separate regions rather than combinations thereof. This observation suggests that the proposed method is sensitive to the discrepancies occurring between distinctly responding regions. The material from this chapter was used in the preparation of a peer-reviewed article now published (GómezLaberge et al., 2008).

\subsection{Space characterisation: voxel cluster contiguity}

The cerebrovasculature perfuses contiguous volumes of tissue via common networks of arterioles (Hamel, 2006). Furthermore, their regulation of CBF is locally undertaken by the constriction and dilation of the cerebral microvasculature. Therefore, neural activity is known to occur in contiguous regions of tissue (Malonek \& Grinvald, 1996; Kuschinsky, 2000; Iadecola \& Nedergaard, 2007). Consequently, the BOLD signal is also expected to emerge as a contiguous spatial distribution of voxels in BOLD fMRI data. Based on this evidence, this spatial criterion seeks clusters containing sets of contiguous voxels. 
The data acquired within the scanner $w[n]=w[x, t]$ are a discrete sampling of the volume field of view over time. Spatially, each sample point can be considered as the volume integral of the MR signal within the enclosing voxel dimensions. Temporally, the centroids are calculated as in equation (5.41) of algorithm 2 (page 76) and, therefore, are membership-weighted linear combinations of the member voxel time sequences. Hence, the clusters reveal how the member voxels are distributed in space as a function of temporal correlation with the centroid. Therefore, for each cluster $X_{i}$, we can define a spatial function that produces the voxel time sequence correlation between each member voxel $x$ and the centroid $v_{i}[t]$

$$
R_{i}[x]= \begin{cases}r\left(x, v_{i}\right) & \text { for } x \in X_{i}, \\ 0 & \text { otherwise }\end{cases}
$$

where $r\left(x, v_{i}\right)$ is the Pearson correlation given by equation (5.45) (page 76). Given $R_{i}[x]$ for $i=1, \ldots, k$, contiguous clusters can be potentially distinguished from sporadic clusters.

This requires that the term contiguity be quantitatively defined. To this end, the following terminology is proposed. Two voxels who share the same face are said to be adjacent, and one unbroken sequence of adjacent voxels is defined as a group. Then, all $\left|X_{i}\right|$ voxels of a cluster can be counted group-wise by the series

$$
\left|X_{i}\right|=1 g_{1}+2 g_{2}+\cdots+M g_{M}
$$

where $g_{j}$ is the number of groups of $j$ adjacent voxels, and the largest group has $M$ adjacent voxels. We consider a group to be contiguous if it contains a number of voxels equal to or greater than some constant $m>1$. Thus, by setting $g_{j}=0$ for $j<m$, we obtain a fraction of $\left|X_{i}\right|$ voxels belonging to contiguous groups

$$
0 \leq \frac{1}{\left|X_{i}\right|} \sum_{j=m}^{M} j g_{j} \leq 1
$$

Finally, to penalise clusters with a large number of groups, say $G_{i}$, the series is also 


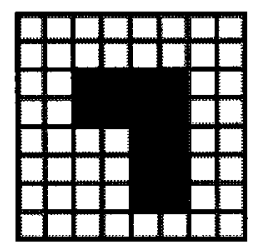

$c=1$

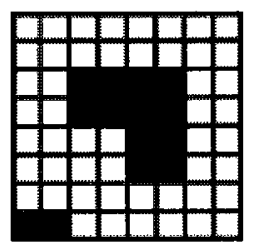

$c=0.86$

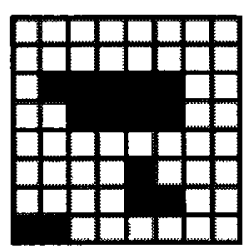

$c=0.43$

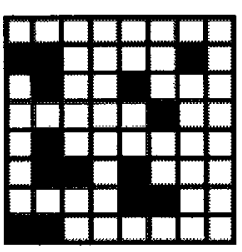

$c=0.21$

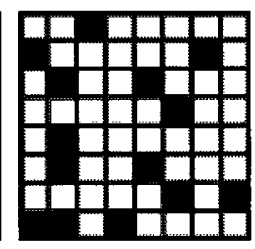

$c=0$

Figure 6.1: Contiguity for a collection of two-dimensional clusters. In each case, the total number of member voxels (black) is $\left|X_{i}\right|=14$, and the smallest group size is set to $m=3$.

normalised by the product $G_{i}\left|X_{i}\right|$ instead of simply by $\left|X_{i}\right|$. With these notions, we define the contiguity of a voxel cluster as follows.

Definition 6. Given a cluster $X_{i}$, its correlation map $R_{i}[x]$, a correlation threshold $0<$ $r_{c}<1$, and an integer $m>1$, the contiguity of the voxels $x \in X_{i}$ with $R_{i}[x] \geq r_{c}$ is given by the series

$$
c=\frac{1}{G_{i}\left|X_{i}\right|} \sum_{j=m}^{M} j g_{j},
$$

where $G_{i}$ is the total number of groups, and the largest group contains $M$ adjacent voxels.

While it is not unusual to expect multiple distinct regions interacting during the execution of a task, we expect this number be small in relation to $k$, compared to a large number of small sporadic regions that are poorly correlated and likely grouped together by chance. This expectation is reasonable according to the objective of identifying distinct anatomical regions per cluster that independently regulate their vascular supply. The properties of contiguity are demonstrated with the following example. Figure 6.1 illustrates the contiguity values obtained from definition 6 with $m=3$ for a fictional set of two-dimensional clusters. For example, the middle cluster has two contiguous groups: one with three voxels, the other with nine voxels. Thus, the contiguity according to the definition is $c=(1 \times 3+1 \times 9) /(2 \times 14)=0.43$. The total number of voxels in each cluster was chosen to be the same: $\left|X_{i}\right|=14$. Notice that the contiguity is bounded between $[0,1]$ and increases with fewer, larger groups. Furthermore, note that the measure is independent of voxel size, as long as $m$ is scaled by the volume ratio between sizes. Finally, the contiguity is also invariant to cluster geometry so that spherical, planar, or linear clusters 
are considered equally.

Before applying the contiguity definition to the set of voxels in each cluster, it is important to consider the nature of cluster membership in terms of correlation. Recall that in algorithm 2 (page 76), the centroid $v_{i}$ from equation (5.41) is a weighted average of the member voxel time sequences, where similar voxel sequences are assigned large membership coefficients $u_{i}(x)$ calculated using equation (5.42).

In neuroimage data, when comparing the cluster centroid with its member voxel time sequences, almost all of the entire range of positive correlation values is typically observed. However, a lower limit must exist where $\min _{x \in X_{i}} R_{k}[x]>0$, since by definition no member voxel can be uncorrelated to the centroid. Therefore, for each cluster, we obtain a bounded function of the contiguity over the domain of correlation coefficients written as $c\left(r_{c}\right)$, where the parameter $0 \leq r_{c} \leq 1$ is a lower limit threshold that only includes voxels with $R_{k}[x] \geq r_{c}$ in the contiguity calculation. Figure 6.2 illustrates the function $c\left(r_{c}\right)$ and the corresponding behaviour of the cluster when $r_{c}$ takes on several values. These images are shown in the radiological convention: as though viewing the transverse section from the subject's feet. Hence, the right hemisphere appears on the left side of the image and vice versa. Notice, in particular, how low contiguity thresholds lead to the admission of spurious voxels that are poorly correlated.

The choice of parameter values for $r_{c}$ and $m$ should be based on the properties of the cerebrovasculature that are known in general and are also observed in the data. In particular, the size of a contiguous voxel group determined by $m$ should be chosen based on the relationship between the voxel size and the known extent of functional hyperemia and spatial smoothing performed during data preprocessing. The threshold $r_{c}$ can be determined based on the behaviour of the function $c\left(r_{c}\right)$ and even, perhaps, on a statistical hypothesis test that would have to account for the known dependence between adjacent voxel correlation values that was demonstrated by the autocorrelation estimates $\hat{\gamma}[\tau]$ from chapter 4. As a first step to demonstrate the performance of this criterion on neuroimage 


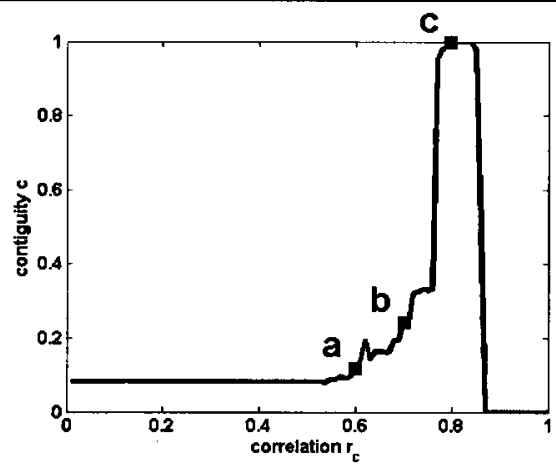

Figure 6.2: Illustration of the effect of $r_{c}$ threshold values on the contiguity function for a typical voxel cluster with colour bar indicating $r\left(x, v_{i}\right)$ on a transverse anatomical overlay shown in radiological convention. Voxels forming a contiguous cluster $X_{i}$ are shown according to the colourmap scale that quantifies $r\left(x, v_{i}\right)$ for contiguity thresholds $r_{c}$ of 0.6 (a, blue square), 0.7 (b, green square), and 0.8 (c, red square). 
data, we set the threshold limit $r_{c}$ equal to the median of the values taken by the contiguity function for each cluster.

\subsection{Time characterisation: causal cross-correlation}

The hand grip response recorded by the experimental apparatus in the visual feedback controlled motor task is an indicator of the temporal relation between the event-related stimuli and the nervous system response. Hence, a comparison is possible between the temporal behaviour of each cluster centroid and the motor response by calculating the cross-correlation function of their time sequences. The centroid time sequence $v_{i}[t]$, of length $T$, for each cluster can be compared to the motor response time sequence $m[t]$, subsampled to match $v_{i}[t]$, according to the following definition for a causal cross-correlation function.

Definition 7. Given a cluster centroid time sequence $v_{i}[t]$, a simultaneously recorded motor response time sequence $m[t]$, subsampled such that $t=0, \ldots, T-1$ correspond to the same time points for both sequences, and a known minimum interstimulus duration $\Delta>1$ measured in time sample units, the causal cross-correlation function is the sequence of length $\Delta$ given by

$$
v_{i} \star m[d]=\sum_{t=0}^{T-1} \tilde{v}_{i}[t+d] m[t], \quad d=0, \ldots, \Delta-1,
$$

where $\tilde{v}_{i}[t]=v_{i}[t \bmod T]$ is the periodic extension of $v_{i}$, and $d$ is the delay of the BOLD signal following the motor response.

The magnitude of $v_{i} \star m[d]$ is meaningful because it is related to the correlation $r\left(\tilde{v}_{i}[t+d], m[t]\right)$ by the formula $v_{i} \star m[d]=(T-1) s_{v} s_{m} r\left(\tilde{v}_{i}[t+d], m[t]\right)$, where $s_{v}$ and $s_{m}$ are the sample standard deviations of the sequences $v_{i}[t]$ and $m[t]$, respectively. This formula follows immediately from the definition of $r(x, y)$ given in equation (5.45) (page 76). The delay $d$ is also important because it models the phase shifts in $v_{i}[t]$ due to the expected delay in functional hyperemia. Time advances $d<0$ are excluded from this model because the stimuli are presented following a randomised interstimulus duration to minimise 


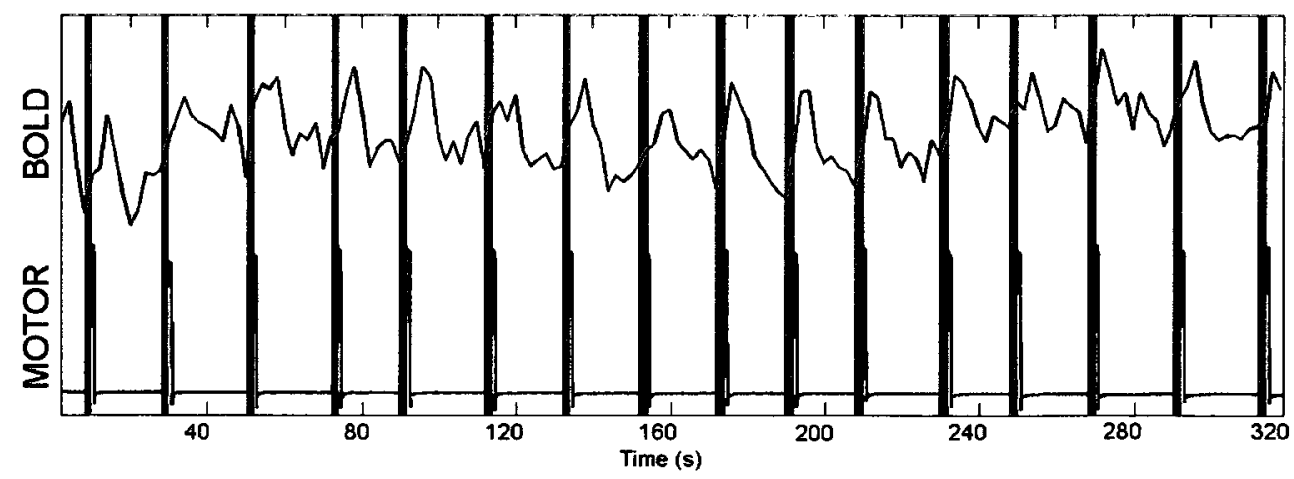

Figure 6.3: Plot of a cluster centroid BOLD response signal (black), the corresponding motor response signal (red), and the event-related stimuli (grey) acquired throughout a neuroimage session from a normal subject.

anticipation of the events. Furthermore, delays larger than the minimum interstimulus duration $d>\Delta$ are also excluded to avoid the incorrect association of a BOLD response with motor responses that precede the latest motor response during an sequence of events. Figure 6.3 illustrates the typical relationship between the event-related stimuli, the motor response, and the BOLD response through the course of one neuroimaging session in a normal subject. The maximum value of $v_{i} \star m[d]$ corresponds to a correlation of $r=0.44$ with a delay of $d=1$, which corresponds to 2 seconds because the MR protocol used $T_{R}=2$ s. The delay is reasonable when looking at the time difference between corresponding peaks in the $v_{i}[t]$ and $m[t]$ signals as well as when comparing with the expected normal response plotted in figure 4.3a (page 57). However, limitations of this approach are also apparent in this example. First, note that the response delay between signals is longer in the early events than in the later events. This effect is not captured by definition 7 , since it considers the entire session rather than each event separately. Secondly, the quality of the BOLD signal is relatively poor compared to the motor signal because it is subject to prominent artefacts caused by scanner noise, minor systemic fluctuations in blood flow, and minor head movements. Thus, the cross-correlation magnitude is influenced by the intensity of these artefactual effects and can lead to a misrepresentation of the average response throughout the session.

This latter point leads to the question of selecting clusters based on the magnitude 
of $v_{i} \star m[d]$, or based on the degree of correlation each cluster has to the motor response signal. As shown previously in chapter 4 , clusters with negatively correlated centroids have been observed in previous BOLD fMRI studies (Goutte et al., 1999; Röther et al., 2002; Roc et al., 2006). Hence, we equally consider clusters with negatively correlated centroids as responsive to the paradigm by selecting clusters with $\left|r\left(\tilde{v}_{i}[t+d], m[t]\right)\right| \geq r_{m}$, where $0<r_{m}<1$ is the correlation threshold between the delayed BOLD and motor sequences that maximise $v_{i} \star m[d]$. While arbitrary thresholds can be set for individual sessions, the variability between subjects, e.g., each having their own degree intercluster dependence, makes this approach unrealistic in general. It seems reasonable for each session, however, that clusters with the highest causal cross-correlations are the most likely regions representing a BOLD response signal. Consequently, in the next chapter, we include all the cluster parameters from a single session together in a session-wide probability model to determine the appropriate threshold values for cluster selection. To demonstrate the performance of this criterion at present, the results shown here are obtained with an arbitrary threshold $r_{m}=0.30$ (whose value is in fact justified in the next chapter) for neuroimage data acquired from normal subjects.

\subsection{Results}

The proposed criteria are applied to neuroimage data to characterise the space-time structure of brain regions that respond to the event-related paradigm. Indeed, the cluster used to generate figures 6.2 and 6.3 is obtained from a normal subject performing the motor task with their right hand, and it is clear that the response is colocalised with the left M1 motor area and exhibits a centroid time sequence that is causally related to the motor response signal.

Figure 6.4 applies these criteria to a different normal subject performing the same motor task with their right hand. The resulting clusters obtained from the proposed 


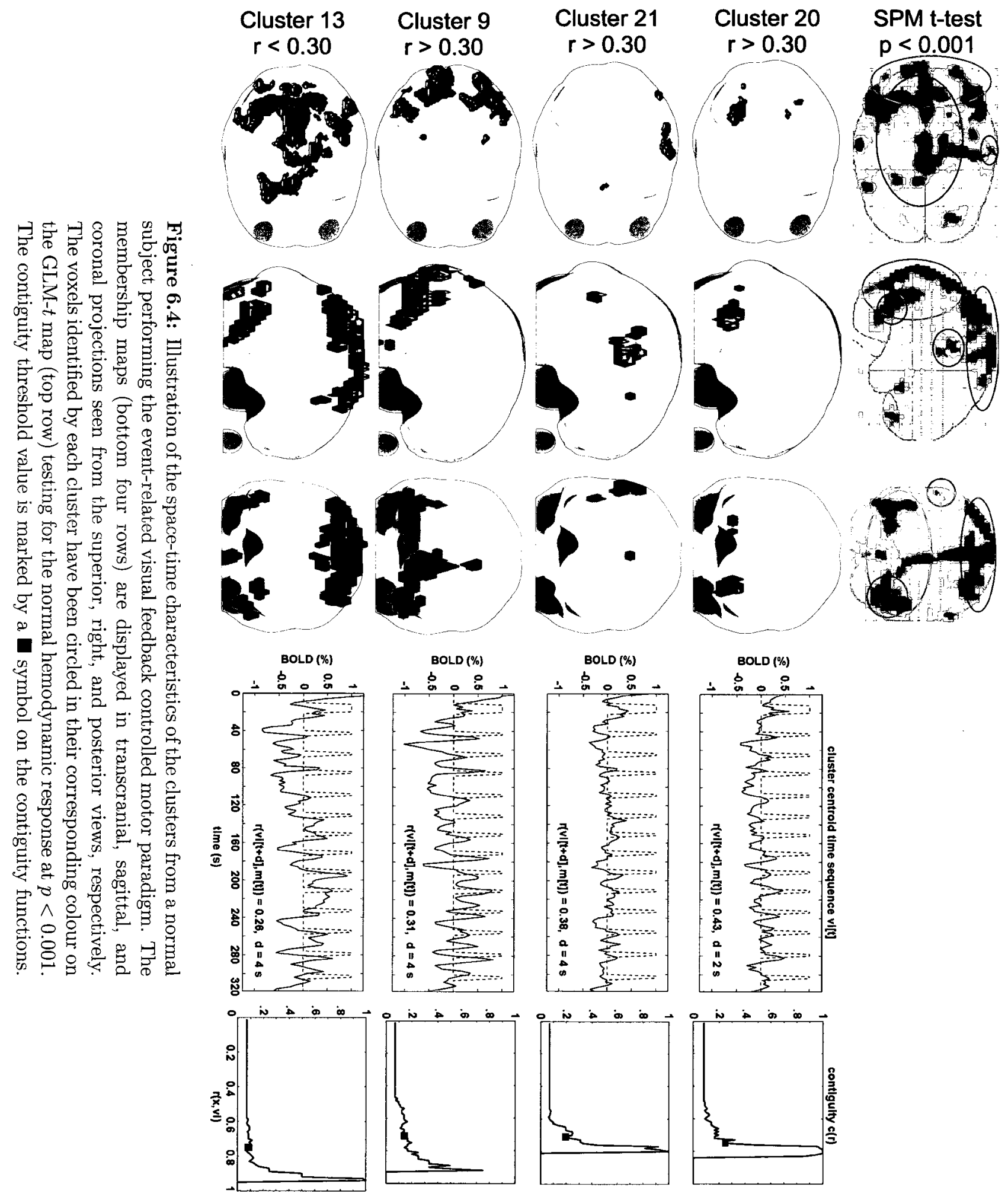


method are compared with the GLM- $t$ map. The most prominent observation between these methods is that the four clusters shown match the GLM- $t$ map when they are superimposed over each other. This is highlighted by colour-coding each cluster with their corresponding regions in the GLM- $t$ map. It is clear that each of these clusters are correlated to the motor signal; however, their centroid time sequences plotted are dissimilar and preferably should not be mixed.

Clusters 20 and 21 are within the right cerebellum and the left M1 motor area, respectively. As discussed in chapter 2, these functional areas are well known to be involved in our event-related paradigm. Clusters 9 and 13, are more likely to be venous signals generated by the accumulation of blood draining through the transverse and superior sagittal sinuses, respectively. Indications of this are based on the colocalisation of clusters 9 and 13 with these prominent venous sinuses, the heightened intensity of their BOLD signals compared to the $\mathrm{M} 1$ response in cluster 21 , and their matching delay and similar time sequences.

Two other interesting observations can be made from this result. First, the amplitude of the BOLD signal in each cluster seems related to the location of the contiguity function peak value. Indeed, the maximum contiguity threshold $r_{c \max }$ that produces a non-zero $c\left(r_{c \max }\right)$ is proportionally related to the SNR of the cluster. To see this, notice that in the noiseless case SNR $\rightarrow \infty$, the voxels are perfectly correlated with the centroid time sequence, which implies that $r_{c \max } \rightarrow 1$. As noise is introduced, the correlations must fall and consequently $r_{c \max }$ must follow. Second, the suspected venous clusters 9 and 13 have a relatively lower contiguity and motor response correlation than clusters 20 and 21. This shows that selecting the threshold $r_{m}$ arbitrarily can easily lead to incorrect cluster selection. This observation motivates the development of an objective method for threshold selection presented in the final chapter on the analysis methods in this thesis. 


\subsection{Discussion}

This chapter proposes a method for the systematic selection of voxel maps identifying potentially activated cerebral territories obtained from the cluster analysis of BOLD fMRI data. Selection criteria were proposed for the automated interpretation and selection of the clusters whose voxels are significantly responsive to the motor response during eventrelated stimuli and occupy contiguous regions in the brain.

Cluster contiguity reveals interesting properties of the spatial structure of the data. The functions $c(r)$ shown in figures 6.2 and 6.4 have the typical shape seen in the majority of the selected clusters we have observed in these studies. That is, for high thresholds, these strongly correlated member voxels are concentrated in one region and hence have a high contiguity. As the threshold is relaxed, weakly correlated members are increasingly admitted and are more so sparsely distributed as $R_{i}[x]$ becomes small. Therefore, spurious clusters can be distinguished from compact ones by comparing their contiguity functions. We also observed how the value $r_{c \max }$ may be useful in estimating SNR throughout clusters. However, while this may be helpful in signal analysis, we must give caution that calculating the SNR value correctly from $r_{c \max }$ in these data is non-trivial because of the intervoxel dependence introduced into the $r\left(x, v_{i}\right)$ data due to the spatial correlations noted in chapter 4.

In an earlier study by Goutte et al. (1999), the causal cross-correlation between centroids and an artificial stimulus signal was recommended as a pre-processing stage. There, the correlation values and corresponding phase delays served as an alternative to neuroimage data in an ordinary $k$-means clustering to reduce the algorithm's sensitivity to the fixed number of clusters and to mitigate scanner noise. Although this may be highly relevant for $k$-means clustering, this method was not used here for two reasons. First, it is critical in our research to relate the cerebrovascular and nervous system responses because of their known uncoupling in stroke. Therefore, we instead correlate the centroids to the motor response. Second, we are not using the original $k$-means clustering which is 
more sensitive to the choice of initial parameters and instead account for noise using the established preprocessing techniques that have so far proven effective for our analyses.

The results shown in this chapter demonstrate how the expected M1 motor region is identified in normal subjects that performed the event-related motor task with their right hand. While, these voxels are also identified by the GLM- $t$ testing for the normal hemodynamic response, the major difference observed is that the proposed method distinguishes between responses while the GLM- $t$ merges them together. In conclusion, these results provide evidence that pattern recognition methods can potentially expose relevant spatial and temporal characteristics of these regions by not mixing identified response signals across separate regions. Such information may be useful in studying functional hyperemia behaviour perturbed by cerebrovascular disease in distinct regions of the brain. 


\section{Chapter 7}

\section{Analysis Methods: Part III Bayesian Hierarchical Model}

\subsection{Introduction}

The analytical research undertaken in the preparation of this thesis is concluded in this chapter. The final stage of analysis is a probabilistic framework that models the structure identified in functional neuroimage data. The central motivation for probabilistic neuroimage analysis revolves around the fact that these data are primarily used for exploratory scientific research with the aim of understanding the substrates of the functional organisation of the brain. Generally speaking, we rely on a probability model to infer the true functional state of the subject from the empirical observations that are made from our data. This statement, however, precludes the selection of a suitable model; a task that first requires the careful examination and characterisation of the observed data as was performed in the previous chapters. Indeed, when studying complex systems and data acquired therefrom, the research to determine a suitable probability model is substantial and, in fact, is a study on the nature of the system itself.

Building on the results from the structural characterisation of the data clusters identified with exploratory pattern recognition, a Bayesian hierarchical probability model 
is proposed to determine the statistical significance of each cluster with respect to the global structure observed throughout the entire data set. It is important to mention that the model presented here is general and does not depend on the specific clustering and parameterisation methods used in this thesis. This modular approach is desirable for two reasons. First, it circumvents the difficulty discussed in chapter 5 of directly integrating statistical methods into the exploratory pattern recognition algorithm, and, second, it allows for the independent innovation and comparison of exploratory pattern recognition and parameterisation algorithms.

This chapter begins by empirically justifying the choice of a Bayesian hierarchical model with a demonstration of the hierarchical organisation of the data and the Gaussian distribution of its temporal correlation structure. Next, the probability theory of the model is developed and is applied to functional neuroimage data. The implementation a Markov chain Monte Carlo (MCMC) technique used to fit the model to the data is described and includes a technique to verify its convergence. The method is validated using simulated BOLD fMRI data and by comparing results with the activation maps generated from a model-based GLM- $t$ test. The specificity of the method is examined using null fMRI data. The results suggest that the proposed method provides an objective framework to identify, and estimate the significance of, distinct BOLD response signals in event-related fMRI. The chapter ends with a discussion of the advantages and limitations of the model. The material presented in this chapter is a novel application of Bayesian data analysis for the exploratory pattern recognition of neuroimage data and is currently being used in the preparation of an article to be submitted for peer-reviewed publication. However, the idea of applying a Bayesian hierarchical model to BOLD fMRI data as a means to infer cluster significance by stimulus-related correlation has been recently (and independently) recommended by Lindquist \& Gelman (2009) as an alternative to avoid the multiple comparison framework used in standard statistical analyses. We also recognised this advantage prior to their publication as well as other important advantages described 


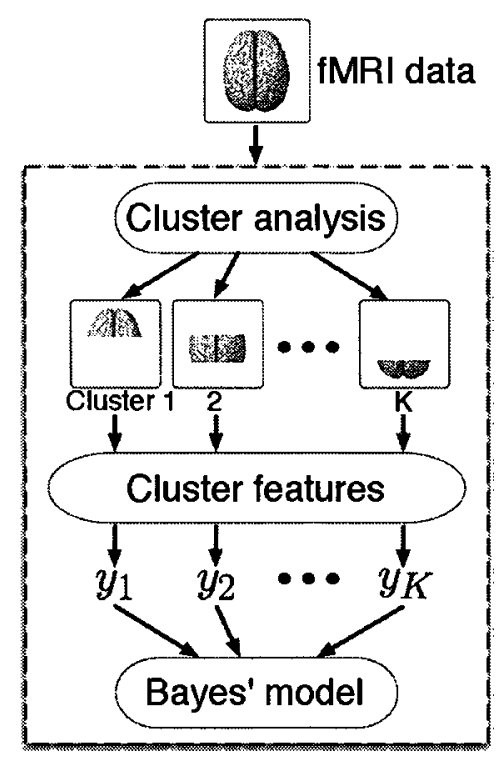

Figure 7.1: Overview of the fMRI analysis stages. The acquired neuroimage data are partitioned by an exploratory pattern recognition algorithm into a set of $k$ clusters. The set of clusters is then parameterised by $k$ features. The features are then used in a Bayesian model to determine the salience of each cluster.

here.

\subsection{Empirical inspection of clustered data}

An overview of the processing stages involved in the analysis of the neuroimage data is given in figure 7.1. Essentially, following cluster analysis and space-time characterisation, the clustered data are parameterised by a feature vector $y_{i}$ for $i=1, \ldots, k$, which can be used as observations within a probability model. For the cluster centroid $v_{i}[t]$ and motor response $m[t]$ time sequences of length $T$, we consider the correlation coefficient $y_{i}=r\left(\tilde{v}_{i}[t+d], m[t]\right)$ obtained by the delay $d$ that maximises the causal cross-correlation function $v_{i} \star m[d]$ from definition 7 (page 92).

To study the empirical distribution of $y$, we consider two questions. At the voxel level, how is the temporal correlation $r(x, m)$ between each voxel $x \in X$ and the motor response $m$ distributed? At the cluster level, how is the correlation $r\left(x, v_{i}\right)$ between each member voxel $x \in X_{i}$ and the cluster centroid $v_{i}$ distributed? Histograms of these 

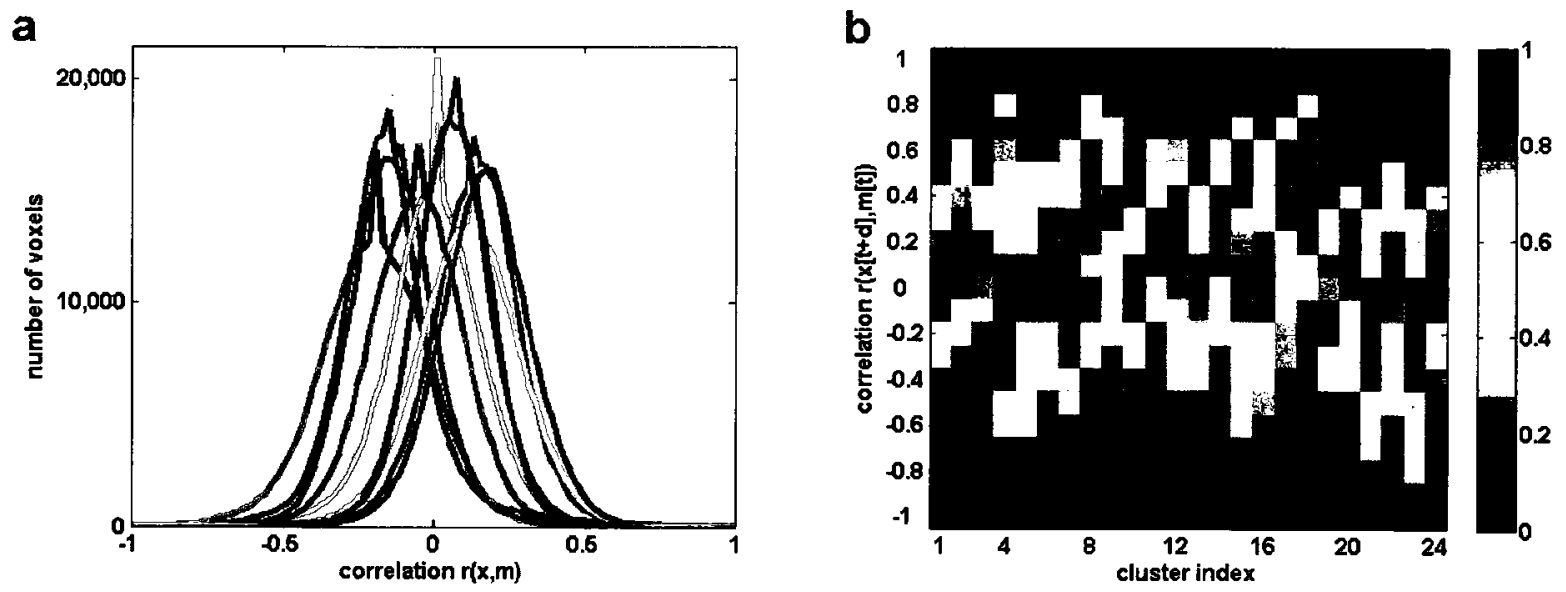

Figure 7.2: Histograms demonstrating the hierarchical and Gaussian form of cluster voxel correlations. a, Guassian-shaped distribution of $r(x, m)$ correlations for ten null fMRI sessions shows how the global signal can be biased in either direction. b, frequency-normalised distribution (colour bar indicates relative frequency) of member voxel correlations (plotted along the ordinate) around their cluster (index along the abscissa) centroid correlation $r\left(v_{i}, m\right)$ within one fMRI session. These histograms demonstrate how motor response correlations are hierarchically distributed and global and cluster levels.

correlations were computed to answer these questions. Figure $7.2 \mathrm{a}$ shows the empirical distribution of $r(x, m)$ for ten null fMRI sessions, and figure $7.2 \mathbf{b}$ shows the superposition of the distributions $r\left(x, v_{i}\right)$ for $i=1, \ldots, k$ from one session. These plots reveal several phenomena. First, at the voxel level, each session produces a Gaussian-shaped distribution of $r(x, m)$ correlations that are not always centred at zero but rather can have a positive or negative bias. Indeed, this bias has been demonstrated in previous fMRI and PET studies (Zarahn et al., 1997; Murphy et al., 2009) resulting in a stimulus-correlated average signal $\hat{\eta}[t]=\langle w[x, t]\rangle_{x}$ referred to as the global signal. Hence, the global signal can be modelled by a Gaussian random variable with mean and variance conditioned by the empirical distribution of $r(x, m)$. Therefore, each voxel correlation $r(x, m)$ can be modelled as a realisation from this random variable. Second, at the cluster level, each session exhibits a hierarchical organisation of correlations $r\left(x, v_{i}\right)$ for $i=1, \ldots, k$, where each cluster produces a Gaussian-shaped distribution centred at $r\left(v_{i}, m\right)$. In conjunction with the bias from the global signal, the voxel correlations are also influenced by the clusters. Consequently, each cluster can be modelled by a Gaussian random variable whose mean 
and variance are conditioned by the empirical distributions of $y_{i}$ and $r\left(x, v_{i}\right)$.

\subsection{Bayesian hierarchical model}

Based on these observations, it is reasonable to explain the correlation data using a model that hierarchically represents the variability in each cluster as well as the global variability, that is, over all clusters combined. For example, in addition to the expected BOLD signal appearing in a subset of localised voxels, other factors such as unrelated physiological and MR scanner effects also cause fluctuations in the data. These can be due to general variations in blood circulation, electronic noise, magnetic field inhomogeneities, or movement, which can affect some or all voxels in the data. Moreover, the intersubject variability shown in figure 7.2a calls to attention the impact that a biased global signal may have on determining cluster significance based on correlation thresholding.

A Bayesian model can account for the bias introduced by the global signal by conditioning the model parameters based on the empirical observations $y$ and their variance. Therefore, we propose a Bayesian hierarchical model to represent the correlative effects of the global signal and of each cluster by model parameters whose values are conditioned on the neuroimage data. The formulation of this model follows the approach of Gelman et al. (2004). The structure of the model parameters is illustrated in figure 7.3. From bottom to top, each of $k$ clusters inputs a vector $y_{i}$ whose elements are empirical measures (e.g., the correlation $\left.r\left(v_{i}, m\right)\right)$ from each cluster. The objective is to determine whether voxels forming cluster $i$ are significantly affected by an underlying response or rather exhibit an unrelated signal. The underlying effect of each cluster is modelled as the parameter $\boldsymbol{\beta}_{i}$ (the true value that $y_{i}$ is measuring). The apex of the structure is the hyperparameter $\boldsymbol{\alpha}$ that models the global signal throughout the data set. The hyperparameter $\boldsymbol{\alpha}$ controls this effect by modelling the influence of the global signal on each cluster via $\boldsymbol{\beta}_{i}$. The following subsections provide the model formulae and explain how the relationship between 


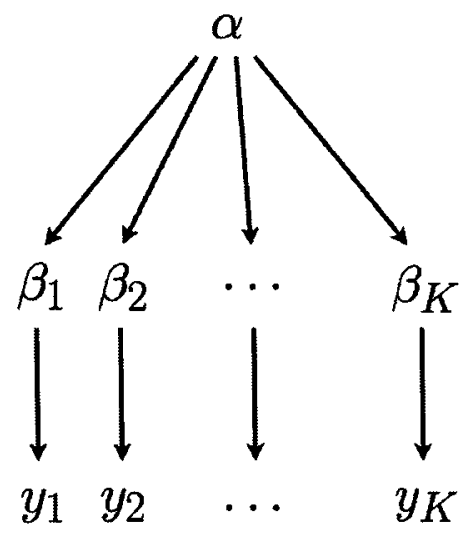

Figure 7.3: Bayesian hierarchical model parameters (adapted from Gelman et al. (2004)) used in the final stage of the analysis. The model parameters $(\alpha, \beta)$ are fit to the data $y$, such that each cluster feature $y_{i}$ is drawn from an underlying signal $\beta_{i}$. In turn, all underlying signals $\beta$ are drawn from a global signal $\alpha$.

the global signal and each cluster is essential in determining cluster significance.

\subsubsection{Joint probability distribution}

The model parameters $\boldsymbol{\alpha}, \boldsymbol{\beta}_{i}$ are Gaussian random variables. The parameter $\boldsymbol{\beta}_{i}$ is distributed with variance $\sigma_{i}^{2}$, estimated directly from the $r\left(x, v_{i}\right)$ data in cluster $i$. The hyperparameter $\boldsymbol{\alpha}$ has variance $\boldsymbol{\tau}^{2}$; these are also random variables, however, less is known about their empirical distributions. Once the mean and variance are described, simulated realisations of the model parameters $\{\alpha, \beta, \tau\}$ given the data $\{y, \sigma\}$ are obtained from the joint posterior distribution

$$
p(\beta, \alpha, \tau \mid y, \sigma) \propto p(\alpha, \tau) p(\beta \mid \alpha, \tau) p(y, \sigma \mid \beta, \alpha, \tau)
$$

where the distributions $p(\cdot)$ are multivariate functions of the vectors $y=\left(y_{1}, \cdots, y_{k}\right)^{\top}$, $\sigma=\left(\sigma_{1}, \cdots, \sigma_{k}\right)^{\top}, \beta=\left(\beta_{1}, \cdots, \beta_{k}\right)^{\top}$, and the scalars $\alpha$ and $\tau$. Thus, the form of the distribution $p(\beta, \alpha, \tau \mid y, \sigma)$ encapsulates the probabilistic structure of the entire model. This distribution is shaped by the functions on the right-hand side of equation (7.1). 


\subsubsection{The likelihood, population, and prior distributions}

The cluster centroid is the sample average of its member voxel time series. Thus, appealing to the central limit theorem, the distribution of the centroid correlation values approaches normality for clusters of many voxels. This approximation is already good for clusters containing over twenty voxels. Hence, given parameter realisations, the likelihood of each data point can be modelled as a Gaussian distribution

$$
y_{i}, \sigma_{i} \mid \beta_{i}, \alpha, \tau \sim \mathrm{N}\left(\beta_{i}, \sigma_{i}^{2}\right) .
$$

In exploratory models, two convenient assumptions exchangeability and Bayesian conjugacy can be made when little is known about the nature of the data. Exchangeability allows us to model the multivariate distributions in equation (7.1) as simple products of their components. Thus, the joint likelihood takes the form

$$
p(y, \sigma \mid \beta)=\prod_{i=1}^{k} p\left(y_{i}, \sigma_{i} \mid \beta_{i}\right) .
$$

Bayesian conjugacy is a type of symmetry in equation (7.1), such that the prior and population distributions have the conjugate parametric form of the likelihood. This implies that the posterior distribution also has the conjugate form of the likelihood. In the case of a Gaussian distributed likelihood as in equation (7.2), conjugacy implies that the population, prior, and posterior densities also be of Gaussian form.

Thus, under the assumption of exchangeability, the joint population distribution takes the product form

$$
p(\beta \mid \alpha, \tau)=\prod_{i=1}^{k} p\left(\beta_{i} \mid \alpha, \tau\right),
$$

where each cluster has a Gaussian random variable describing its population

$$
\boldsymbol{\beta}_{i} \mid \alpha, \tau \sim \mathrm{N}\left(\alpha, \tau^{2}\right) .
$$


Finally, since nothing is known about $\boldsymbol{\alpha}$ and $\boldsymbol{\tau}$ a priori, a noninformative distribution for the joint prior $p(\alpha, \tau)$ is appropriate. Indeed, if we assume the uniform distributions $p(\alpha \mid \tau) \propto 1$ and $p(\tau) \propto 1$, then, the joint prior is also uniform

$$
p(\alpha, \tau)=p(\alpha \mid \tau) p(\tau) \propto 1
$$

\subsection{Gibbs sampling of the posterior density}

Only in simple cases can $p(\beta, \alpha, \tau \mid y, \sigma)$ be computed with Bayes' rule analytically, as in equation (7.1). Here, we consider a more general approach that is immediately applicable to hierarchical models that are not necessarily Gaussian. The approach simulates posterior density realisations of the model parameters using the MCMC technique known as the Gibbs sampler. This requires factoring equation (7.1) into the alternate form

$$
\begin{aligned}
p(\beta, \alpha, \tau \mid y, \sigma) & =p(\beta \mid \alpha, \tau, y, \sigma) p(\alpha, \tau \mid y, \sigma) \\
& =p(\beta \mid \alpha, \tau, y, \sigma) p(\alpha \mid \tau, y, \sigma) p(\tau \mid y, \sigma)
\end{aligned}
$$

so that realisations can be simulated from each term starting with the rightmost density to give $\tau$, then $\alpha$, and finally $\beta$. These MCMC simulations begin with rough estimates of the parameters based on the data, and with each iteration the Markov chain is guaranteed to approach the true distribution of the parameters.

Gelman et al. (2004) describe a method for simulating batch realisations of $k+$ 2 variables $\left\{\beta_{1}, \cdots, \beta_{k}, \alpha, \tau\right\}$ for each iteration, hence, yielding one simulated point of the joint posterior distribution $p(\beta, \alpha, \tau \mid y, \sigma)$. To simulate realisations, we require the conditional density functions on the right-hand side of equation (7.7). Starting with the conditional posterior distribution of $\boldsymbol{\tau}^{2}$, we have the following result.

Lemma 1 (Raiffa \& Schlaifer (1961); Gelman et al. (2004)). Let $\left(y_{i}, \sigma_{i}^{2}\right)$ for $i=1, \ldots, k$ be an exchangeable data set described by a conjugate Bayesian hierarchical model with 
non-informative hyperparameters $\boldsymbol{\alpha}$ and $\boldsymbol{\tau}$, and a set of Gaussian parameters $\boldsymbol{\beta}_{i} \mid \alpha, \tau \sim$ $\mathrm{N}\left(\alpha, \tau^{2}\right)$ corresponding to each data point. Then the Gibbs sampler estimate of the conditional posterior distribution of the variance $\tau^{2}$ follows a scaled inverse- $\chi^{2}$ distribution, written as

$$
\tau^{2} \mid \beta_{i}, \alpha, y \sim \operatorname{Inv}-\chi^{2}\left(k-1, \hat{\tau}^{2}\right),
$$

where $\hat{\tau}^{2}$ is the unbiased variance estimate based on the realisations $\alpha$ and $\beta_{i}$ given by

$$
\hat{\tau}^{2}=\frac{1}{k-1} \sum_{i=1}^{K}\left(\beta_{i}-\alpha\right)^{2} .
$$

\section{Proof.}

For a generic random variable $\boldsymbol{\theta}>0$, the scaled inverse- $\chi^{2}$ distribution is written as $\boldsymbol{\theta} \sim \operatorname{Inv}-\chi^{2}\left(\nu, s^{2}\right)$ and has the exponential form

$$
p\left(\theta \mid \nu, s^{2}\right)=\frac{(\nu / 2)^{\nu / 2}}{\Gamma(\nu / 2)} s^{\nu} \theta^{-(\nu / 2+1)} e^{\nu s^{2} /(2 \theta)},
$$

with two parameters: the degrees of freedom $\nu>0$ and the scale $s>0$. We can establish that the random variable $\tau^{2} \mid \beta_{i}, \alpha, y$ is scaled inverse- $\chi^{2}$ by using the conjugate prior density and letting $\boldsymbol{\theta}=\boldsymbol{\tau}^{2}$. From equation (7.2), the likelihood $\boldsymbol{\beta}_{i} \mid \alpha, \tau^{2}$ is

$$
p\left(\beta_{i} \mid \alpha, \tau^{2}\right) \propto\left(\tau^{2}\right)^{-(k-1) / 2} \exp \left(-\frac{(k-1) \hat{\tau}^{2}}{2 \tau^{2}}\right),
$$

where the unbiased average is written as

$$
\hat{\tau}^{2}=\frac{1}{k-1} \sum_{i=1}^{k}\left(\beta_{i}-\alpha\right)^{2} .
$$

Recall that $\tau$ has a noninformative prior density $p(\tau) \propto 1$; therefore, $p\left(\beta \mid \alpha, \tau^{2}\right)$ has $k-1$ degrees of freedom rather than $k$. The conjugate prior of equation (7.11) has the form $\tau^{2} \sim \operatorname{Inv}-\chi^{2}\left(\nu, s^{2}\right)$; thus, we write it as

$$
p\left(\tau^{2}\right) \propto s^{\nu}\left(\tau^{2}\right)^{-(\nu / 2+1)} e^{-\nu s^{2} / 2 \tau^{2}} .
$$

Computing the product $p\left(\tau^{2}\right) p\left(\beta \mid \alpha, \tau^{2}\right)$ gives the posterior density

$$
\begin{aligned}
p\left(\tau^{2} \mid \beta, \alpha\right) & \propto\left[\left(\frac{s^{2}}{\tau^{2}}\right)^{\nu / 2+1} e^{\nu s^{2} / 2 \tau^{2}}\right]\left[\left(\tau^{2}\right)^{-(K-1) / 2} e^{-(K-1) \hat{\tau}^{2} / 2 \tau^{2}}\right] \\
& \propto\left(\tau^{2}\right)^{-(\nu+K-1) / 2+1} e^{-\left(\nu s^{2}+(K-1) \hat{\tau}^{2}\right) / 2 \tau^{2}} .
\end{aligned}
$$

From this expression, one can recognise that

$$
\tau^{2} \mid \beta, \alpha \sim \operatorname{Inv}-\chi^{2}\left(\nu+K-1,\left[\nu s^{2}+(K-1) \hat{\tau}^{2}\right] /[\nu+K-1]\right) .
$$


Finally, notice that the noninformative prior $p(\tau) \propto 1$ implies that $\nu=0$, and when substituted back into equation (7.15) establishes equation (7.8).

QED

Moving on to the second term from the right in equation (7.7), we seek the conditional posterior distribution of $\alpha$. It is not hard to see that this distribution is simply the sample average and sample variance of the $\beta_{i}$ terms

$$
\boldsymbol{\alpha} \mid \beta, \tau \sim \mathrm{N}\left(\frac{1}{k} \sum_{i=1}^{k} \beta_{i}, \frac{\tau^{2}}{k}\right)
$$

Finally, the conditional posterior distribution of $\boldsymbol{\beta}_{i}$ is obtained based on the exchangeability assumption, since the multivariate density functions for equations (7.7) and (7.2) have product forms. Thus, computing the product $p(\beta \mid \alpha, \tau) p(y, \sigma \mid \beta, \alpha, \tau)$ using equations (7.3) and (7.4) yields the following result.

Lemma 2 (Gelman et al. (2004)). Let $\left(y_{i}, \sigma_{i}^{2}\right)$ for $i=1, \ldots, k$ be an exchangeable data set described by a conjugate Bayesian hierarchical model with noninformative hyperparameters $\boldsymbol{\alpha}$ and $\boldsymbol{\tau}$, and a set of Gaussian parameters $\boldsymbol{\beta}_{i} \mid \alpha, \tau \sim \mathrm{N}\left(\alpha, \tau^{2}\right)$ corresponding to each data point. Then the Gibbs sampler estimate of the conditional posterior distribution of each $\boldsymbol{\beta}_{i}$ is the Gaussian random variable

$$
\boldsymbol{\beta}_{i} \mid \alpha, \tau, y_{i}, \sigma_{i} \sim \mathrm{N}\left(\hat{\beta}_{i}, V_{\beta_{i}}\right)
$$

whose mean and variance parameters are based on hyperparameter realisations

$$
\begin{aligned}
\hat{\beta}_{i} & =\frac{y_{i} / \sigma_{i}^{2}+\alpha / \tau^{2}}{1 / \sigma_{i}^{2}+1 / \tau^{2}}, \\
V_{\beta_{i}} & =\frac{1}{1 / \sigma_{i}^{2}+1 / \tau^{2}} .
\end{aligned}
$$

\section{Proof.}

The conditional posterior distribution of each realisation $\beta$ is

$$
\begin{aligned}
p(\beta \mid \alpha, \tau, y, \sigma) & =p(\beta \mid \alpha, \tau) p(y, \sigma \mid \beta, \alpha, \tau) \\
& =p(\beta \mid \alpha, \tau) p(y, \sigma \mid \beta),
\end{aligned}
$$


where

$$
\begin{aligned}
& p(\beta \mid \alpha, \tau)=\frac{1}{\tau \sqrt{2 \pi}} e^{-(\beta-\alpha)^{2} / 2 \tau^{2}}, \\
& p(y, \sigma \mid \beta)=\frac{1}{\sigma \sqrt{2 \pi}} e^{-(y-\beta)^{2} / 2 \sigma^{2}} .
\end{aligned}
$$

Thus, the product gives

$$
p(\beta \mid \alpha, \tau, y, \sigma)=\frac{1}{2 \pi \sigma \tau} \exp \left[-\frac{1}{2}(\underbrace{\frac{(\beta-\alpha)^{2}}{\tau^{2}}+\frac{(y-\beta)^{2}}{\sigma^{2}}}_{T})\right] .
$$

The term $T$ is quadratic in $\beta$

$$
T=\left(\frac{1}{\sigma^{2}}+\frac{1}{\tau^{2}}\right)\left\{\beta^{2}-2\left(\frac{\frac{y}{\sigma^{2}}+\frac{\alpha}{\tau^{2}}}{\frac{1}{\sigma^{2}}+\frac{1}{\tau^{2}}}\right) \beta+\left(\frac{\frac{y^{2}}{\sigma^{2}}+\frac{\alpha^{2}}{\tau^{2}}}{\frac{1}{\sigma^{2}}+\frac{1}{\tau^{2}}}\right)\right\} .
$$

By completing the square and absorbing the third term into the normalising factor for the distribution, one recognises the Gaussian form with mean and variance as claimed

$$
\begin{aligned}
& \hat{\beta}=\frac{\frac{y}{\sigma^{2}}+\frac{\alpha}{\tau^{2}}}{\frac{1}{\sigma^{2}}+\frac{1}{\tau^{2}}}, \\
& V=\frac{1}{\frac{1}{\sigma^{2}}+\frac{1}{\tau^{2}}} .
\end{aligned}
$$

QED

In summary, given the data $\left(y, \sigma^{2}\right)$, equations (7.8), (7.16), and (7.17) yield a batch realisation for the respective conditional posterior random variables $\boldsymbol{\tau}, \boldsymbol{\alpha}$, and $\boldsymbol{\beta}$ from each iteration of the Gibbs sampler. As noted, these are MCMC simulations that ultimately estimate realisations by iteratively converging toward the true joint posterior distribution $p(\beta, \alpha, \tau \mid y, \sigma)$. Therefore, to ensure that the estimates are sufficiently close to the target distribution and, thus, can be treated as true realisations, we will require a means to check for convergence. The following simulation algorithm encapsulates the entire Gibbs sampler with convergence verification.

Algorithm 3 (Gibbs sampler (Gelman et al., 2004)). Let $\left(y_{i}, \sigma_{i}^{2}\right)$ for $i=1, \ldots, k$ be $a$ data set described by a Bayesian hierarchical model with random variables $\boldsymbol{\tau}, \boldsymbol{\alpha}$, and $\boldsymbol{\beta}$. Choose two positive integers, such as, $G=10$ the number of Gibbs samplers running in parallel, and $2 N=2,000$ the number of realisations from each sampler, and one small number, such as $\epsilon=0.001$. Then a total of $G N=10,000$ batch realisations are obtained 
by the doing the following for each of the $G$ samplers:

1. Set the initial values for each $\beta_{i}=y_{i}$ and compute the initial value for $\alpha=\left\langle\beta_{i}\right\rangle$.

2. Simulate a realisation of $\tau$ using equation (7.8)

$$
\tau^{2} \mid \beta_{i}, \alpha, y \sim \operatorname{Inv}-\chi^{2}\left(k-1, \hat{\tau}^{2}\right),
$$

which can be implemented by first making random draws from a $\chi^{2}$ random variable with $k-1$ degrees of freedom, $X=\chi_{k-1}^{2}$, and then setting $\tau^{2}=(k-1) \hat{\tau}^{2} / X$.

3. Simulate a realisation of $\alpha$ using equation (7.16)

$$
\boldsymbol{\alpha} \mid \beta, \tau \sim \mathrm{N}\left(\frac{1}{k} \sum_{i=1}^{k} \beta_{i}, \frac{\tau^{2}}{k}\right)
$$

with the latest realisations of $\tau$ and $\beta$.

4. Simulate a realisation for each $\beta_{i}$ using equation (7.17)

$$
\boldsymbol{\beta}_{i} \mid \alpha, \tau, y_{i}, \sigma_{i} \sim \mathrm{N}\left(\hat{\beta}_{i}, V_{\beta_{i}}\right)
$$

with the latest realisations of $\alpha$ and $\tau$.

5. Store the batch of latest realisations $\left\{\tau, \alpha, \beta_{1}, \cdots, \beta_{k}\right\}$.

6. Repeat steps 2-5 until $2 N$ batches are obtained.

7. Discard the first $N$ batches from each Gibbs sampler (considered "burn in" samples).

8. Compute the potential scale reduction given by

$$
\hat{R}=\sqrt{\frac{1}{W}\left(\frac{N-1}{N} W+\frac{1}{N} B\right)},
$$

where $W$ and $B$ are the within-sampler and between-sampler variance of the remaining $G N$ realisations.

9. If the inequality $|\hat{R}|<1+\epsilon$ is satisfied, then these $G N$ realisations are convergent and represent realisations from the true joint posterior distribution of the Bayesian hierarchical model.

Now equipped with a convergent MCMC sampling of the joint probability distribution, we can calculate the significance of each cluster with respect to the global signal by computing Bayes' error between the distributions of the hyperparameter $\boldsymbol{\alpha}$ and the cluster parameters $\boldsymbol{\beta}_{i}$, for each $i=1, \ldots, k$. Bayes' error is the probability that a sample 
is incorrectly classified as being drawn from a particular distribution and represents the optimal error rate in sample classification between the two distributions. Geometrically, it is the overlapping area between the tails of both distributions. Therefore, a cluster feature $y_{i}$ is significant with probability of error $p$ when the Bayes' error between the distributions of the global signal $\boldsymbol{\alpha}$ and the cluster parameter $\boldsymbol{\beta}_{i}$ is less than $p$. We illustrate as a summary graph the area overlap between each $\boldsymbol{\beta}_{i}$ and $\boldsymbol{\alpha}$ by generating a corresponding set of boxplots arranged horizontally whose box and whiskers extend vertically and show (by convention) the $5 \%$ and $95 \%$ quantiles of each distribution. When the boxplot of a given $\boldsymbol{\beta}_{i}$ does not overlap with the boxplot of $\alpha$, then that cluster has a distribution of correlation values that are significantly different from the rest of the data set and potentially represent a stimulus-response cluster with probability of error $p<0.05$.

\subsection{Results}

Summary results from the proposed method are shown in figure 7.4. The joint posterior density $p(\beta, \alpha, \tau \mid y, \sigma)$ is illustrated in $7.4 \mathrm{a}$ by a set of box plots representing the MCMC distributions of the parameters $\alpha, \beta_{1}, \ldots, \beta_{k}$ arranged horizontally, much like the correlation histogram of figure $7.2 \mathrm{~b}$. The inter-quartile range (IQR) of the voxel-paradigm correlations are shown by the box height; the whiskers extend another 1.5 IQR beyond the box. A low Bayes' error corresponds to a cluster whose box plot does not overlap with that of $\alpha$ shown in grey. Boxes marked with an asterisk have $p<0.05$. The figure shows that the simulated data are partitioned into 24 clusters, where $\beta$ corresponding to clusters 3,4 , and 5 are significantly different from $\alpha$. The three response-signals seem to be well distinguished by the voxel maps in figure $7.4 \mathrm{~b}$. The averaged voxel time series are shown in figure $7.4 \mathbf{c}$. The correlations and response delay pairs $\left(r_{i}, d_{i}\right)$ for clusters 3,4 , and 5 , are $(0.78,8),(0.78,6),(-0.87,4)$, respectively. The correlations are higher than typically seen in fMRI due to the inclusion of signal voxels with SNR $>1$, but the delays (in sec- 
onds) match those seen in figure 4.3a (page 57). The stability of the proposed method was verified by three repeated analyses on three simulated data sets. In all cases the same three clusters were identified from a range of $[24,30]$ clusters whose correlation coefficient estimate differed on average by $3.8 \times 10^{-4}$ within a data set, and by 0.034 between data sets.

Results using the GLM- $t$ testing for the normal hemodynamic response at $p<0.001$ are computed for comparison. Two, one-tailed tests are done to detect a significant increase or decrease in voxel intensity, referred to as "activation" or "deactivation", respectively. Summary results from the GLM- $t$ method are shown in figure 7.5. Using the paradigm that generated the data shown in figure $4.3 \mathbf{b}$ (page 57), only signal 2 is identified as an activation as shown in figure 7.5a. The deactivation finds no signal voxels. Signals 1 and 3 , however, are identified by advancing the paradigm by 4 seconds $(p[n] \rightarrow p[n+2])$ as suggested by the time-to-peak of the simulated signals in figure $4.3 \mathbf{a}$. The significance threshold for the GLM- $t$ is $p<0.001$. This value was chosen ad hoc to approximate the significance threshold of the proposed method so that the voxel maps in figures 7.4 and 7.5 may be visually compared. Indeed, the structure of the $t$-maps shown are similar to the $r$-maps in figure 7.4 from the proposed method, as expected according to Golay et al. (1998). Thus, similar results between methods are observed as their respective significance thresholds, indicated by the white bar across the grey scales, increase proportionately. Both methods have comparable sensitivity. This can be seen by recording the SNR of the weakest signal voxel correctly identified in each of the 24 image slices. The weakest signal voxel correctly identified by the proposed method has median $\mathrm{SNR}=0.51$ and worst case $\mathrm{SNR}=0.78 ;$ similarly, the GLM- $t$ method has median SNR $=0.56$ and worst case SNR $=0.83$.

The only major difference seen between the methods is related to specificity, namely, the ability to distinguish between the three response signals. In general, the proposed method seems capable of distinguishing various signals automatically, while the GLM- 

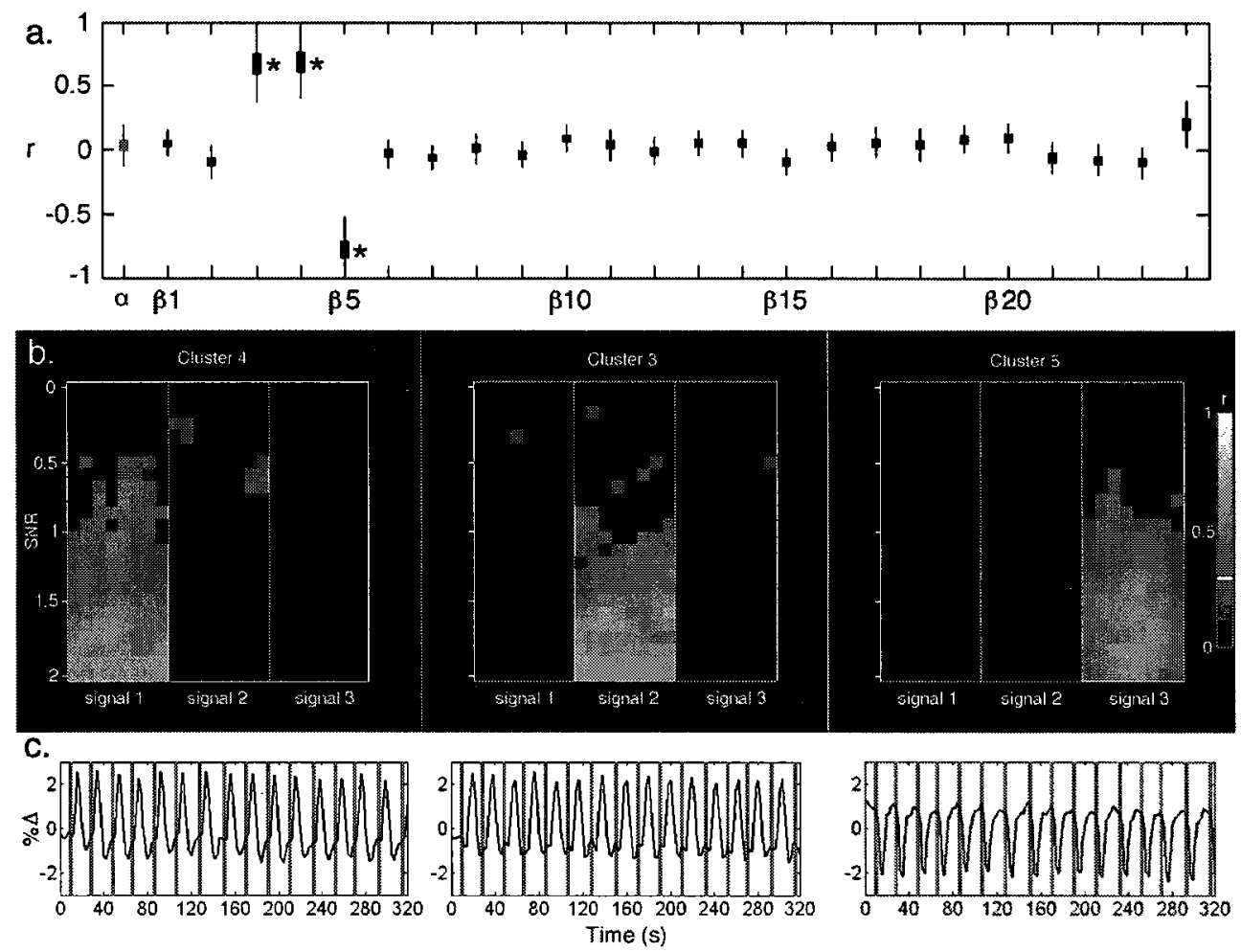

Figure 7.4: Simulation results from the proposed method. a, Box plots of paradigmcluster correlation values from the entire data set $\alpha$ and each cluster $\beta_{i}(i=1, \ldots, k)$. The ${ }^{*}$ symbol denotes clusters that are significantly different from $\alpha$ with $p<0.05$. b, Significant voxel-cluster correlation maps for clusters 3-5 (membership threshold indicated by white bar on scale). c, Averaged voxel time series (events indicated by vertical grey bars).
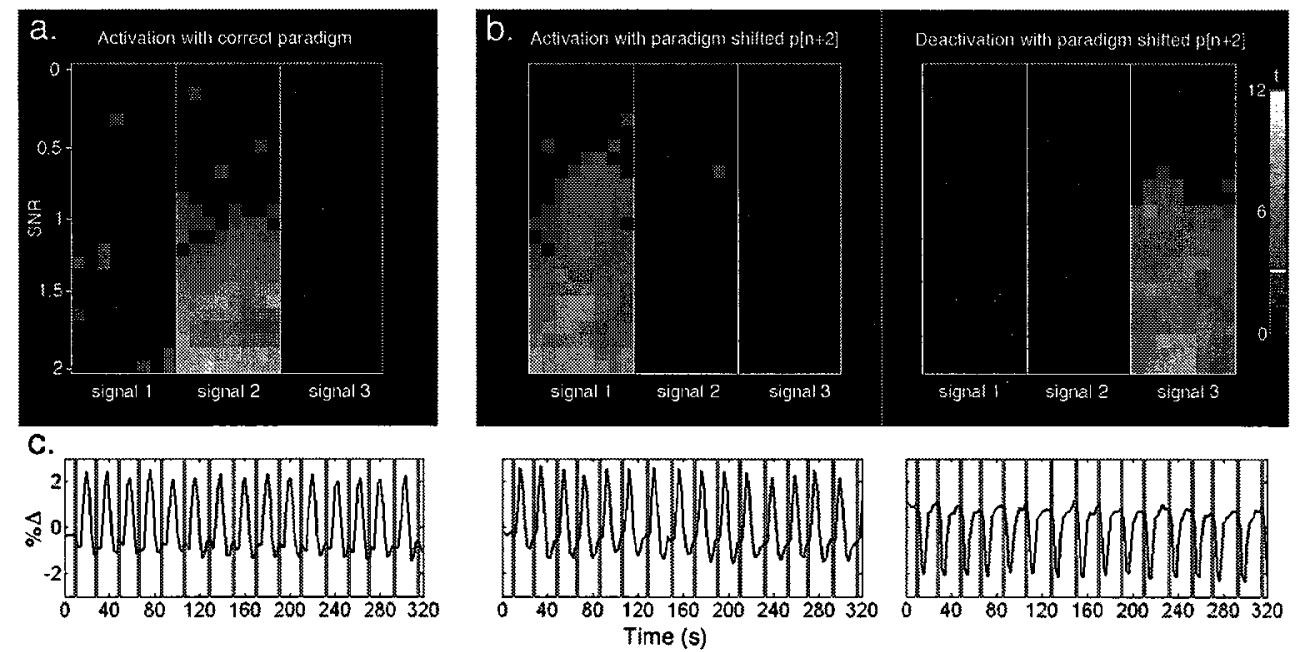

Figure 7.5: Simulation results from the GLM- $t$ method. a, Significant voxelparadigm $t$-maps obtained from the correct paradigm. $\mathbf{b}$, Significant voxel-paradigm $t$-maps obtained from the paradigm advanced by 4 seconds ( $t$-threshold indicated by white bar on scale). c, Averaged voxel time series (events indicated by vertical grey bars). 
$t$ requires informed adjustments of the paradigm to achieve similar results. Even with the necessary adjustments, the GLM- $t$ method does not discern signals 1 and 2 as well as the proposed method. In this simulation, the proposed method seldom misclassifies these signal voxels. The worst case misclassification by the proposed method occurred in a voxel with $\mathrm{SNR}=0.78$. The strongest signal voxels misclassified by the GLM- $t$ method, however, occur at higher signal strengths in voxels with a median $\mathrm{SNR}=0.56$, and worst case SNR $=2.00$. This difference is apparent when comparing the SNR level of the confounded voxels in cluster 3 of figure $7.4 \mathrm{~b}$ with those of figure $7.5 \mathrm{a}$. Both methods, however, identified signal 3 with perfect specificity.

Finally, we applied the proposed method to a set of ten null fMRI data sessions to determine if clusters could be falsely selected. To do this, we applied the event-related stimulus sequence shown in figure 4.3b (page 57) to these data, and the Bayesian model concluded that none of the clusters were related to the stimulus. For example, the largest stimulus-related centroid correlation observed in these clusters had a coefficient of 0.18 , which led to a probability of being significantly different from the global signal of correlation $\alpha=0.06 \pm 0.14$ (mean $\pm \mathrm{SD}$ ) with corresponding Bayes' error $p=0.21$.

\subsection{Discussion}

The application of exploratory algorithms to fMRI data has been hindered by the difficulty in determining the statistical significance of the analysis results. However, the benefit of using such algorithms to study the complex regulatory mechanisms of the cerebrovasculature and its interaction with the nervous system may be unprecedented. This chapter proposes an exploratory method for event-related fMRI, which uses the Bayesian hierarchical model as a statistical framework to infer the significance of each identified region. Our simulation results show comparable behaviour between the exploratory method and the GLM- $t$ over a wide range of physiologically plausible SNR levels. These results also 
demonstrate that the proposed method can discriminate between distinct response signals without a priori knowledge of their dynamics.

The proposed method offers three advantages in analysing event-related data over methods based on hypothesis test statistics. First, the fuzzy $k$-means algorithm used is independent of the stimulus paradigm and, therefore, is not biased by the unequal sample sizes between stimulus-response and rest measurements inherent in event-related designs. The reliability of hypothesis test statistics, on the other hand, is considerably affected by unequal sample sizes, especially when samples exhibit variance heterogeneity (Howell, 1992). Indeed, the GLM performs best with blocked designs (Worsley \& Friston, 1995) and otherwise may require a correction strategy.

Second, the Bayesian hierarchical model accounts for the effect of the global signal that has been shown by Zarahn et al. (1997) to bias statistical inference. While correction strategies have been proposed for model-based methods (Zarahn et al., 1997; Desjardins et al., 2001; Macey et al., 2004), this has not been addressed in exploratory methods to our knowledge.

Finally, although only response signals that have been reported in the literature were used in our simulation study, the proposed method is not signal dependent and may be used to discover arbitrary response signals. This is important in event-related fMRI as short stimuli (under 4 seconds) may evoke considerably nonlinear responses (Boynton et al., 1996; Vazquez \& Noll, 1998), which are more difficult to model a priori.

We have previously described, in the preceding chapter, selection criteria that are informative of the temporal and spatial structure of voxel clusters from fMRI data. There, we used the arbitrary threshold $\left|r_{m}\right|<0.30$ for cluster selection. To justify this choice, the aggregate correlation coefficient for the significant and insignificant clusters in our fMRI results were computed, yielding $\bar{r}_{\text {accept }}=0.41 \pm 0.17(n=22)$ and $\bar{r}_{\text {reject }}=0.15 \pm 0.08$ $(n=239)$, respectively. Therefore, our data suggest that the decision boundary may indeed lie near $\left|r_{m}\right|=0.30$. However, care is required when considerable global signal 
correlation is present in individual subjects. A recent article by Murphy et al. (2009) investigates the effect of the global signal and correction strategies in detail.

In conclusion, the reproducibility and comparability of results from the proposed exploratory method have been demonstrated on simulated data and on human null fMRI data. The results suggest that the proposed method is a suitable approach to separately identify distinct, physiologically plausible BOLD responses within event-related fMRI sessions. 


\section{Chapter 8}

\section{Imaging Motor Deficits After Stroke: Space-Time Structure of the BOLD Signal}

\subsection{Introduction}

Understanding the regulatory mechanisms of cerebral blood flow and its dysfunction in disease is a central topic in the neurological scientific literature. Ample evidence now exists indicating that factors related to age, disease, and medication can alter cerebrovascular regulation (Attwell \& Iadecola, 2002; D'Esposito et al., 2003; Burke \& Bührle, 2006; Girouard \& Iadecola, 2006; Kocharyan et al., 2008). As a consequence, these factors, many of which may not be readily apparent, will also significantly affect the BOLD signal. The recurring message from these studies is that an analysis inferring neuronal activity from an a priori model of the BOLD response signal can be easily misinterpreted in the presence of these confounding factors. Therefore, fMRI may be better suited as an exploratory technique used to identify and discriminate between differing BOLD responses throughout the brain.

The analytical methods developed throughout the thesis are now applied to neuroimage data acquired during the event-related visual feedback controlled motor task from normal and ischemic stroke populations. First, the normal and stroke participants are enu- 
merated and described. Second, the results obtained from each stage and component of the analysis are given, including the motor responses, neuroimage data, pattern recognition, cluster significance within the Bayesian model, and their space-time characterisation. Finally, the identified brain regions responding to the event-related paradigm from each group are presented in aggregate, compared, and interpreted. Interesting individual cases that demonstrate the differences between groups are also shown. The chapter ends with a discussion on the quality of the data, the performance of the method, and the conclusions drawn from the results and based on the current body of knowledge in the literature. The data and results shown here are currently being used in the preparation of an article to be submitted for peer-reviewed publication.

\subsection{Description of participants}

A summary of the human subjects participating in this study is given here. The full details of the neuroimage and subject participation protocols are given in sections 3.3 and 3.4.

\subsubsection{Normal subjects}

The group of participants representing the normal subject population were admitted if i) they were between the ages of 20 and 65 during the study, ii) they gave informed consent in accordance with the OHREB, and iii) they did not fit any of the following exclusion criteria:

1. Contraindication to MRI as per The Ottawa Hospital criteria

2. Pregnancy

3. Inability to perform the required task

4. Diabetes, uncontrolled hypertension, chronic obstructive pulmonary disease, congestive heart failure, or other significant concurrent medical or surgical disease

5. Prior symptomatic neurological disease resulting in deficit, cognitive changes, or impairment of function 
Table 8.1: Participants in the normal group. Subject 2 declared that he is taking medication to control his hypertension.

\begin{tabular}{|c|c|c|c|}
\hline Subject code & Gender & Age & Medication \\
\hline \hline Normal 1 & male & 38 & none \\
\hline Normal 2 & male & 57 & atorvastatin, irbesartan \\
\hline Normal 3 & male & 37 & none \\
\hline Normal 4 & female & 27 & none \\
\hline Normal 5 & female & 33 & none \\
\hline Normal 6 & male & 52 & none \\
\hline Normal 7 & male & 29 & none \\
\hline
\end{tabular}

6. Seizure in past 12 months

7. Migraine in past week

8. History of alcohol or drug abuse

9. Inability to tolerate MRI (claustrophobia, anxiety)

Seven normal subjects of $39 \pm 11$ years of age (mean, SD) were enrolled as a sample from the "normal" population. During the 4 hours prior to their participation, the subjects did not consume any caffeinated products, any tobacco products, and did not perform physical exercise. During the 12 hours prior to their participation, they did not consume any alcoholic beverages. No issues were identified during the sessions and after asking the subjects whether they experienced any difficulties or abnormalities during their participation. Their details are given in table 8.1. In order to match the quantity of data from the stroke patients, two neuroimage sessions were selected from each subject, yielding a total of 14 sessions forming the normal group data set.

\subsubsection{Stroke patients}

Four stroke patients of $73 \pm 10$ years of age were enrolled as a sample from the unilateral ischemic stroke population with motor deficits that are part of an ongoing multi-centre clinical trial. Their inclusion/exclusion criteria pertinent to this study can be summarised 
Table 8.2: Participants in the ischemic stroke group.

\begin{tabular}{|c|c|c|c|l|c|c|}
\hline $\begin{array}{c}\text { Subject } \\
\text { code }\end{array}$ & Gender & Age & $\begin{array}{c}\text { Ischemic } \\
\text { hemisphere }\end{array}$ & $\begin{array}{l}\text { Infarct } \\
\text { description }\end{array}$ & 3 weeks & 3 months \\
\hline \hline Patient 1 & female & 80 & right & $\begin{array}{l}\text { subcortical frontal lobe } \\
\text { in MCA territory }\end{array}$ & no & yes \\
\hline Patient 2 & male & 61 & left & $\begin{array}{l}\text { cortical lesions in pre- } \\
\text { and post-central gyrii }\end{array}$ & yes & yes \\
\hline Patient 3 & male & 83 & right & $\begin{array}{l}\text { entire frontal lobe } \\
\text { (grey \& white matter), } \\
\text { superior temporal gyrus } \\
\text { in MCA territory, insula, } \\
\text { external capsule, } \\
\text { corpus striatum }\end{array}$ & yes & yes \\
\hline Patient 4 & male & 67 & right & $\begin{array}{l}\text { superior temporal gyrus } \\
\text { in MCA territory, insula, } \\
\text { external capsule, } \\
\text { corpus striatum }\end{array}$ & yes & yes \\
\hline
\end{tabular}

as follows. Each patient or their surrogate gave the informed consent to participate, in accordance with the OHREB. These patients have had recently suffered an acute ischemic stroke involving the cerebral cortex that resulted in hemiparesis. This is their first stroke, and they do not have past or current additional complications, including those of neurological and cardiovascular nature. They do not have an ongoing history of drug/alcohol abuse. When it was possible, the patients were invited to participate at two nominal time points: 3 weeks, and 3 months post-stroke. These visits are referred to henceforth as " 3 weeks" and "3 months," respectively. While the data acquisition and analysis were performed, the investigators were blinded to the patient's medication records. Their details are given in table 8.2 .

Two neuroimage sessions per patient visit were selected testing the ipsilesional hand, and the contralesional hand, yielding a total of 14 sessions forming the stroke group. Figure 8.1 summarises the cerebral infarct of the stroke patients and plots their motor responses acquired during the neuroimage sessions, along with a comparison obtained 
Notes:

- The target force is $\mathbf{2 5 \%}$ MVC in all sessions

-Ordinate scale ranges from $0-50 \%$ MVC unless labeled otherwise

Legend:

\begin{tabular}{|l|}
\hline - left hand \\
right hand \\
- event duration
\end{tabular}

Normal
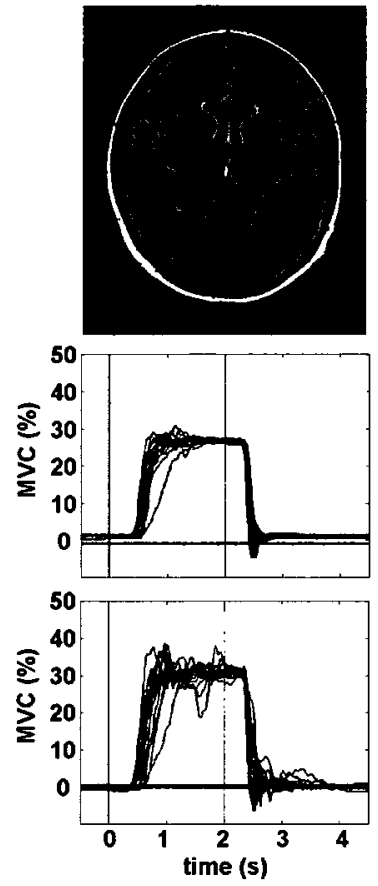

Patient 3
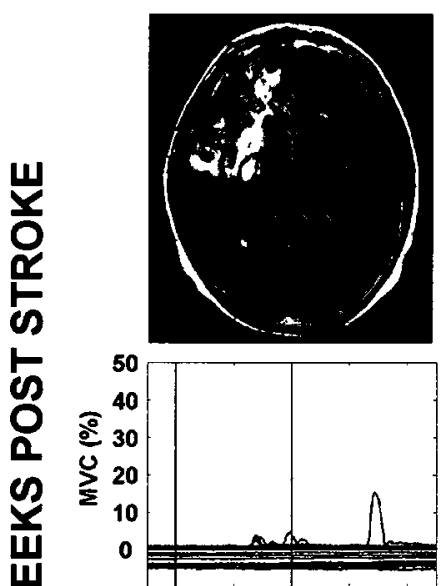

$\sum$

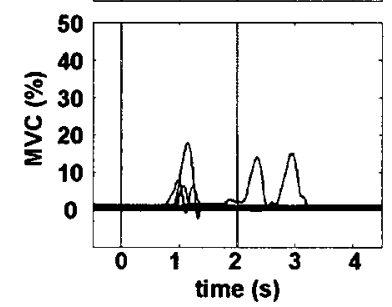

Patient 4
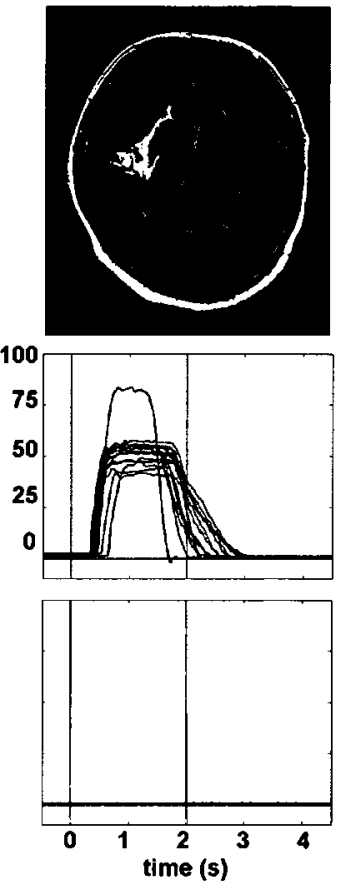

Patient 1

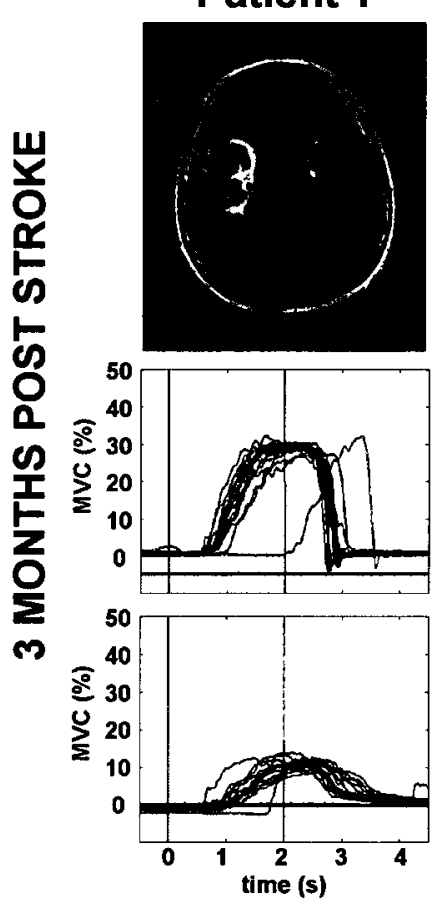

Patient 2
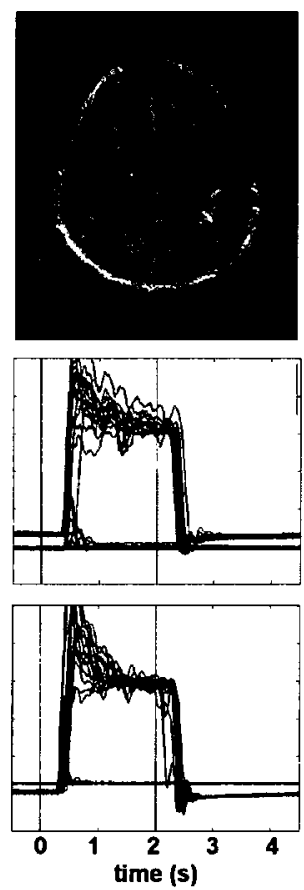

Patient 3
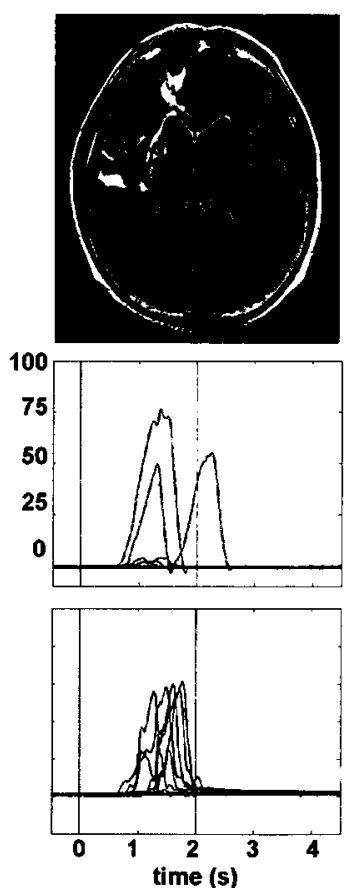

Patient 4
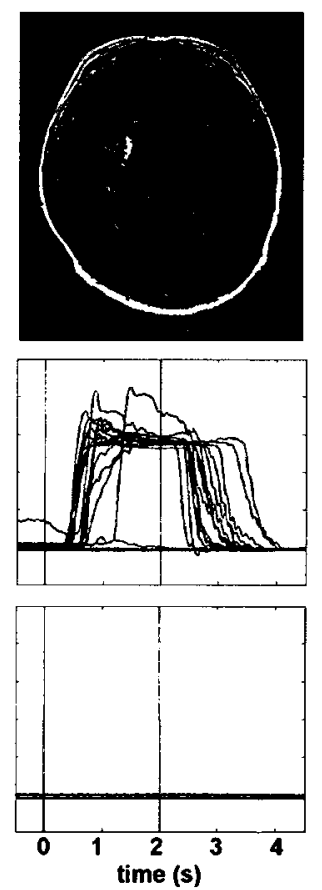

Figure 8.1: Summary showing the structural MRI (radiological convention) and motor responses from the stroke patients in comparison to a normal subject. The motor response signals from both hands are superimposed over each event occurring between the vertical grey lines shown. These data for each subject were acquired during two neuroimage sessions testing the right hand (top graph) and left hand (bottom graph). 
from a normal subject. The structural MRI are shown in radiological convention. These data reveal several outstanding features about our stroke patients and emphasises the variability of impairment resulting from stroke. The ischemic regions vary considerably across this group, including white matter infarct in the right middle cerebral artery (MCA) territory in patient 1 , small focal lesions on the left SMC in patient 2, extensive damage throughout the right frontal lobe and right corpus striatum in patient 3 , and extensive damage in the right insular and right striatal regions in patient 4.

Correspondingly, the severity and nature of the impairments also vary substantially, and introduce challenges in data management and analysis as well as in the interpretation of the results. In particular, patients 1 and 3 were unable to remain still during the sessions and inadvertently made large, sudden head movements being on the order of several millimetres (despite comfortable head restraints). These movements caused major artefacts in the neuroimage data and required that sections containing such artefacts be removed during analysis. Patient 3 also showed a complication of motor and cognitive deficits that resulted in a form of motor agnosia. That is, the patient believed that he was responding well to the stimuli when, in fact, he did not respond to most stimuli and very weakly to few stimuli. Patient 2 was able to perform the task well with both hands, however, would often inadvertently perform mirror movements with the hand that was not being tested. The striatal infarct in Patient 4 resulted in complete plegia in his contralesional hand. Because we could not acquire a motor signal from that hand, we had to make special adjustments to the paradigm to allow the patient to participate in the study. Notably, we asked that the patient imagine moving his hand during events in hopes of observing the presence of a volition-related SMC response. 


\subsection{Results}

\subsubsection{Motor response data}

The motor response signals provide us with an observable that is directly related to the nervous system response to the event-related stimuli. Hence, to probe the neurovascular relationship in this paradigm, we used them in the space-time characterisation of the identified BOLD signal patterns. In addition to using the motor signals directly in our analytical method, we provide a qualitative description of them here by comparing the contraction grip strength, timing, and accuracy to the event stimuli in both hands during each session.

All normal subjects performed the task similarly and with perfect event response accuracy. That is, they responded to every event in a consistent and timely manner using tested hand. Furthermore, they never squeezed the hand grip device between events, and the non-tested hand always remained still throughout the session. Hence, no mirror movements or stimulus unrelated movements of any kind were observed. The normal responses shown in figure 8.1 are characteristic of the group. The dominant hand produced very consistent responses, except during the first event where a slow time-to-target, which is is visible in figure 8.1. For example, the usual delay after the event onset and offset is roughly $500 \pm 50 \mathrm{~ms}$. The time-to-target is roughly $250 \mathrm{~ms}$ and remains quite steady for the event duration. While there is a noticeable difference in the precision in the non-dominant hand, it still retains similar timing characteristics.

The results from each stroke patient is considerably different from the normal group as well as from the other patients in the stroke group. Table 8.3 summarises the main characteristics that were qualitatively observed in the motor response data used in the analysis. In general, the event response accuracy of the stroke patients was good and, if possible, improved in both hands during their second visit. Excluding the sessions testing the plegic hand of patient 4 , the average accuracy across the group in the visit at 3 weeks 


\begin{tabular}{|c|c|c|c|c|c|c|c|}
\hline 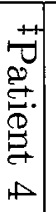 & 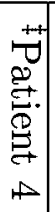 & $\begin{array}{l}\overrightarrow{7} \\
\stackrel{0}{0} \\
\stackrel{0}{0} \\
\stackrel{8}{+} \\
\omega \\
\omega\end{array}$ & 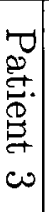 & 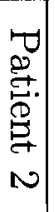 & 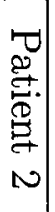 & 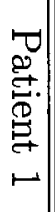 & 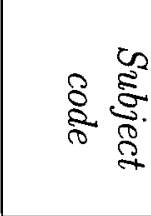 \\
\hline 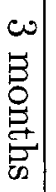 & 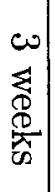 & 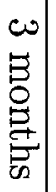 & 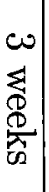 & 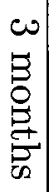 & 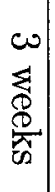 & 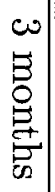 & S: \\
\hline 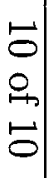 & $\begin{array}{l}\stackrel{\oplus}{\oplus} \\
\stackrel{+}{\oplus} \\
\stackrel{\oplus}{\oplus}\end{array}$ & $\begin{array}{l}- \\
0 \\
\infty \\
\infty\end{array}$ & $\begin{array}{l}\overrightarrow{0} \\
0 \\
\stackrel{+}{0} \\
\vec{N}\end{array}$ & 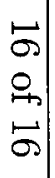 & $\begin{array}{l}\overrightarrow{0} \\
0 \\
\$ \\
\sigma\end{array}$ & 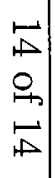 & 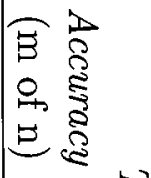 \\
\hline$\Rightarrow$ & $\Rightarrow$ & $\Rightarrow$ & $\Leftarrow$ & $\Rightarrow$ & $\Rightarrow$ & $\|$ & 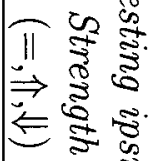 \\
\hline 0 & 0 & $\bullet$ & 0 & $\rightarrow$ & $\oplus$ & 10 & 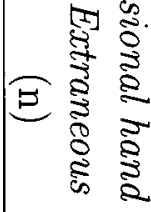 \\
\hline 0 & 0 & 0 & 0 & $\vec{\Delta}$ & $\omega$ & 0 & 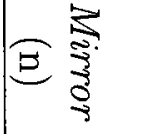 \\
\hline $\begin{array}{l}0 \\
\circ \\
\stackrel{\infty}{\sigma}\end{array}$ & $\begin{array}{l}0 \\
0 \\
\stackrel{0}{2} \\
\sigma\end{array}$ & $\begin{array}{l}\mathrm{c} \\
0 \\
\mathrm{~m} \\
\mathrm{~m}\end{array}$ & $\begin{array}{l}+ \\
\stackrel{+}{\circ} \\
\oplus\end{array}$ & $\begin{array}{l}\vec{\sigma} \\
\stackrel{+}{+} \\
\vec{\sigma}\end{array}$ & 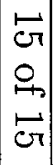 & $\begin{array}{l}0 \\
0 \\
0 \\
0\end{array}$ & 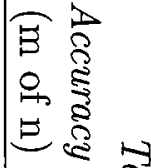 \\
\hline 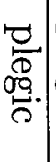 & 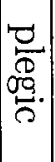 & $\|$ & $\Leftarrow$ & $\Rightarrow$ & $\Rightarrow$ & $\Leftarrow$ & 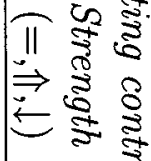 \\
\hline 0 & 0 & $\mapsto$ & $\omega$ & $\oplus$ & $-v \mid$ & 10 & 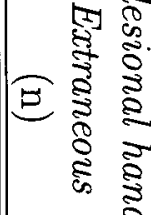 \\
\hline$\circ$ & 0 & 0 & o & $\checkmark$ & 0 & 0 & 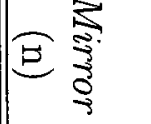 \\
\hline
\end{tabular}

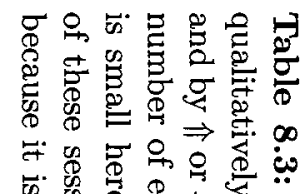

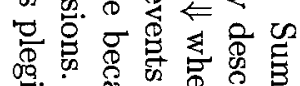

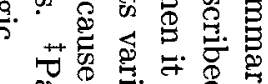

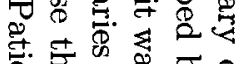

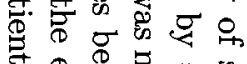

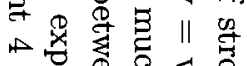

空合

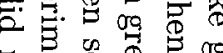

草要惫. 席

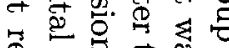

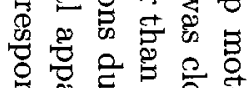

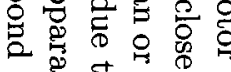

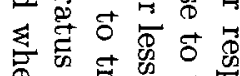

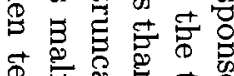

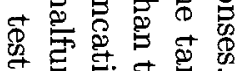

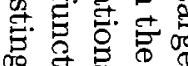

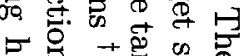

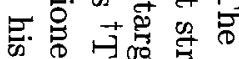

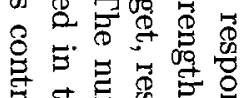

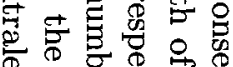

. क :

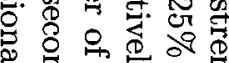

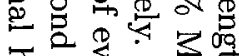

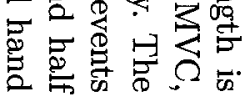


post-stroke is $92 \%$, which includes the outlier session where patient 3 only responded to 4 of the 9 events. During the visit at 3 months post-stroke, the accuracy improved to $98 \%$ with only one event being missed across the group by patient 3 . Indeed, these event response results suggest that the paradigm is suitable for stroke patients with a wide range of motor deficits. Furthermore, the improvement in accuracy between the visits is more likely to reflect the effects of rehabilitation than long-term learning, since the two sessions were over two months apart. Interestingly, the patients have a tendency to respond with more strength than required to meet the target in 10 of the 14 sessions. Since the grip strength was measure relative to their MVC acquired during the calibration and training before neuroimaging, it is possible that the patients were in fact not making their utmost effort during the MVC calibration. However, this effect is not seen in the normal group. Another plausible explanation is that the patient's exhibit a loss of fine hand control that is necessary to achieve the sub-maximal grip strength target.

In addition to the event responses, the patients 2 and 3 occasionally performed extraneous contractions between events. This highlights the importance of using the motor signal instead of the stimulus time sequence in neuroimage analysis. Patient 2 also often performed mirror movements, which are visible in figure 8.1. Thus, we repeated data analysis using the non-tested hand signal to determine whether distinct contralateral SMCrelated BOLD signal was detectable. Finally, in the case of patient 4, who has a plegic contralesional hand, we used a surrogate motor response signal that was obtained by convolving the event pulse train with signal 1 from figure 4.3 (page 57).

\subsubsection{Neuroimage data}

No problematic distortions due to infarction atrophy were observed in the preprocessed neuroimages acquired from the stroke group. Interestingly, however, a prominent image distortion of the anatomy appears in patient 2 due to a small extracerebral paramagnetic implant on the right side of the skull. The paramagnetic implant attenuated the MR 


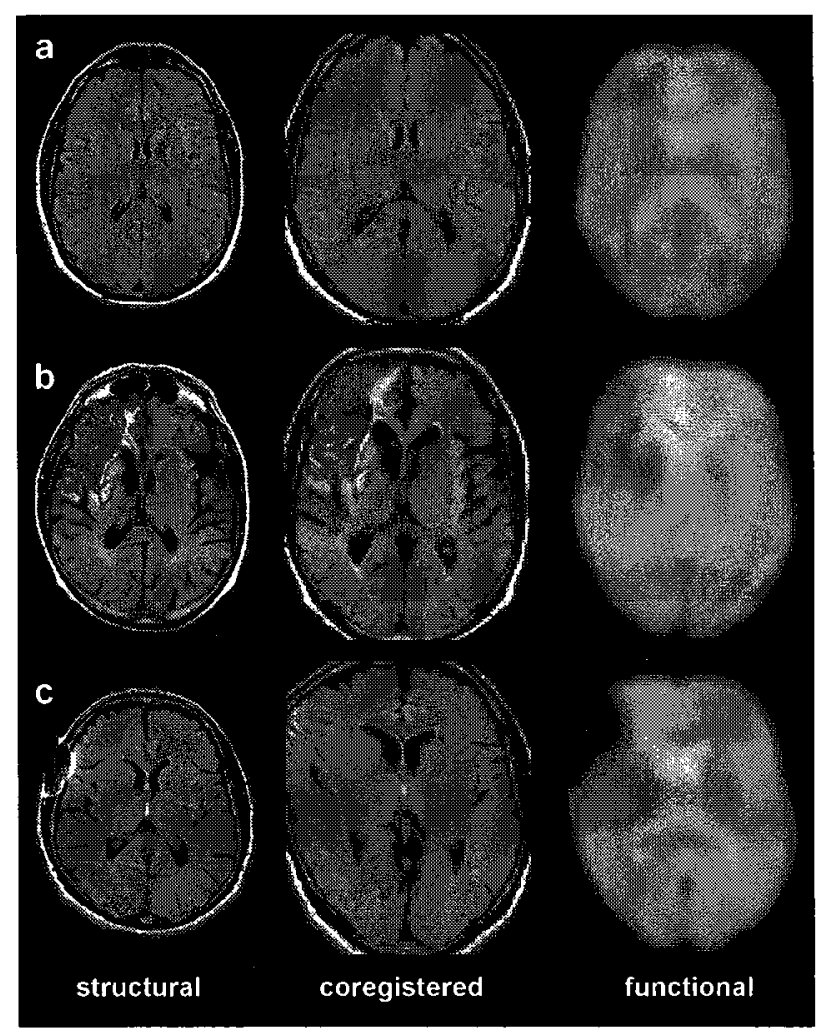

Figure 8.2: Demonstration of the neuroimage quality in a normal subject (a), and in the presence of a large stroke infarction (b) and extracerebral paramagnetic implant (c). A considerable distortion in the anatomy of the coregistered and functional images in row $\mathbf{c}$ is appreciable.

signal in the $\mathrm{T}_{2}$ FLAIR structural image, and is distorted after coregisteration to the EPI functional images. Figure 8.2 compares the extent of these anatomical distortions to a normal subject. As shown in $8.2 \mathrm{c}$, the coregistered structural image overlaps quite well with the regions spared from paramagnetic attenuation in the functional images. In this case, the extent of the region affected by attenuation was greater in the functional image than in the structural one. Therefore, we decided to leave the data intact, since artificially repairing the artefact in the $\mathrm{T}_{2}$ FLAIR and EPI images would at best restore geometry in a region where the BOLD signal could not be measured. In general, we found that the coregistration routine to be adequate for handling image distortions due to infarction atrophy and minor head motion. Paramagnetic implants can potentially lead to problematic attenuation of the functional signal. However, in our case, the spared image region was smallest in the functional image, and we were still capable of reasonably 
coregistering the corresponding regions in the structural image.

Head motion in the normal subjects was always smaller than the EPI voxel dimensions; therefore, the full image sequence data $T=160$ were used in the analyses. The stroke patients, however, were not always motionless. In particular, many images from patients 1 and 3 had to be excluded from analysis because the motions were well beyond the voxel dimensions and were often sudden and reoccurring. When excluding images, we attempted to use as much data as possible without splicing together disjoint images sequences. All but one session retained at least 8 events and were over half of the full image sequence, i.e., with $T>80$. Unfortunately, one session retained only 5 events and was shortened to $T=63$ due in part by head motion and also by an unexpected malfunction in the experimental apparatus where visual controlled feedback was lost after $t>100$. The malfunction was caused by an subtle error in the control software that was introduced during an update in the software code. The problem was resolved after the session and did not reoccur afterward. In general, we found that the detection and compensation of head motion performed by the realignment routine used was adequate except in the case of compensating for large motions that were greater than twice the image voxel size. We verified the detection results from the realignment routine by generating videos of the image data and visually marking time points when motion took place. Moreover, we found this video inspection technique to be very useful in confirming the presence of large movements that were not adequately removed by the realignment routine.

\subsubsection{Bayesian hierarchical model}

The Bayesian hierarchical model was fit to each of the 14 sessions from the normal and stroke groups using the MCMC Gibbs sampler (algorithm 3, page 110). We verified that in every session the MCMC simulated parameters converged to the joint posterior probability density of the clustered neuroimage data according to the value of the potential scale reduction factor described in the algorithm. Figure 8.3 shows the distribution of the 


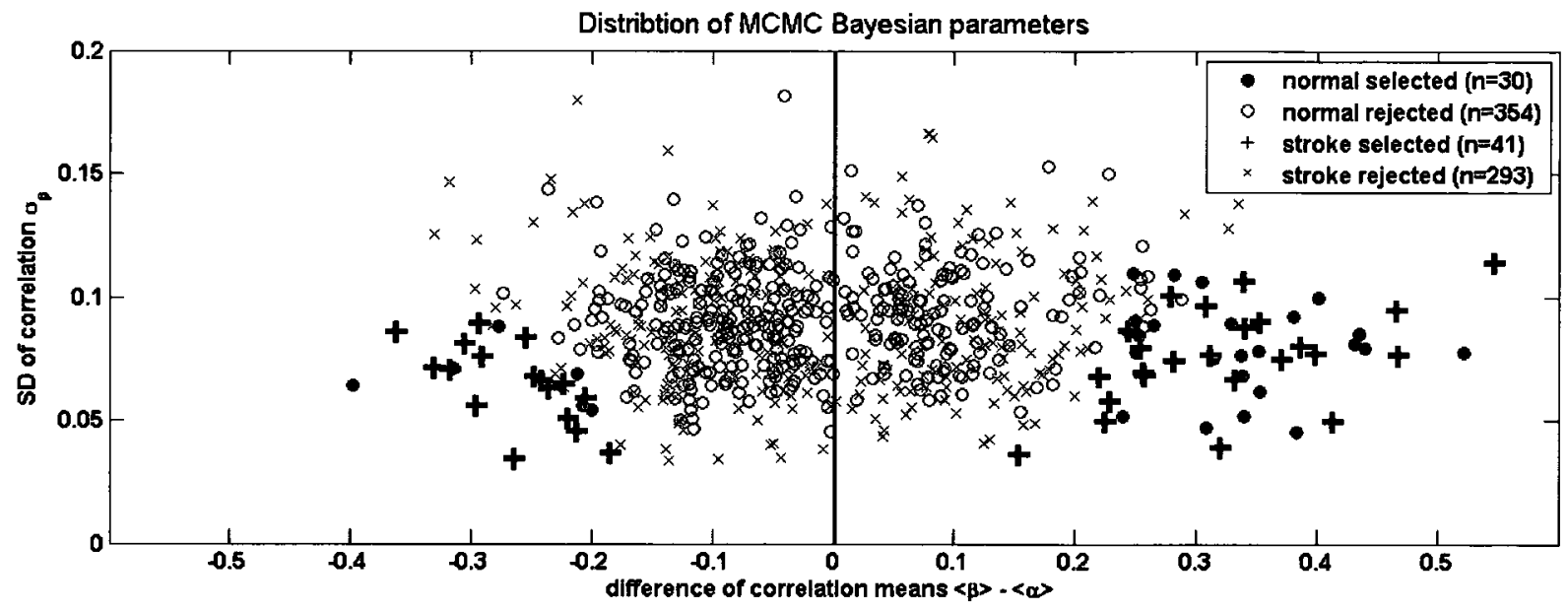

Figure 8.3: Graph of the distribution of the MCMC simulated Bayesian hierarchical model parameters for every cluster identified in the normal (black circles) and stroke (red crosses) groups. Each point in the graph represents the difference of means between the cluster and global signal correlations (plotted along the abscissa) and the cluster's SD of correlations (plotted along the ordinate). The boldfaced points were selected as representing a BOLD signal response to the event-related paradigm.

model parameters for every cluster in all 28 sessions from both groups. For each cluster, the difference between the mean values $\langle\boldsymbol{\beta}\rangle$ and $\langle\boldsymbol{\alpha}\rangle$ is plotted along the abscissa, and the corresponding $\mathrm{SD}$ of $\boldsymbol{\beta}$, written as $\sigma_{\beta}$ is plotted along the ordinate. This graph illustrates how the model represents each cluster in terms of two factors: how different the cluster correlation is from the global signal, and with what certainty this difference is not a random occurrence. According to this graph, the decision boundary for cluster selection appears to be a linear relationship such that the absolute difference $|\langle\boldsymbol{\beta}\rangle-\langle\boldsymbol{\alpha}\rangle|$ must grow linearly in proportion to the SD in $\boldsymbol{\beta}$ for a cluster to be selected. As expected, the decision boundary for positive and negative correlation is symmetric about the origin with parametric form $y=k|x|$, for $k \approx 0.4$.

The rejected clusters from both groups are similarly distributed and well-centred around the global signal $\boldsymbol{\alpha}$. However, this is not the case for the selected clusters across groups. In the normal group, the majority of the selected clusters are positively correlated with few selected clusters being anticorrelated. While the stroke group has a similar distribution of positive correlations, it also has far more anticorrelated clusters. This suggests the presence of negative BOLD signals in our stroke group data. A second 

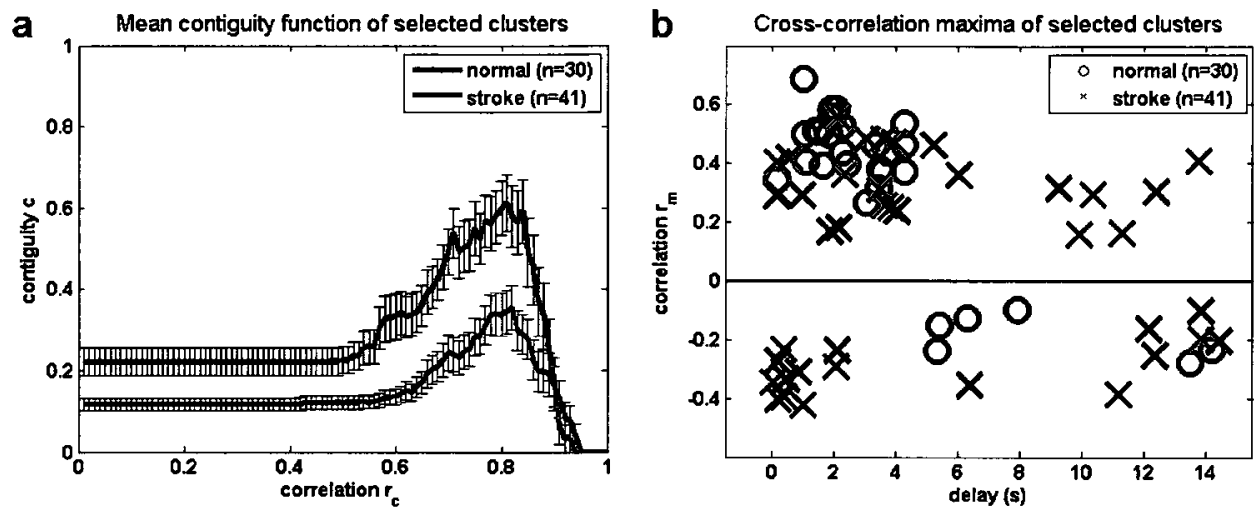

Figure 8.4: Characterisation of the space-time structure of the BOLD signal identified in the normal (black) and stroke (red) groups. a, Plot of the mean contiguity function and its standard error for the selected clusters representing a BOLD response signal. b, Graph of the causal cross-correlation maxima $\left(r_{m}, d\right)$ for the selected clusters representing a BOLD response signal. In order to distinguish the delay values (discretised as $T_{R}$ multiples), they were jittered using random noise uniformly distributed over $[-1,1]$ seconds.

contrast between the groups seen in this figure is the number and proportion of selected clusters. In the normal group, 384 clusters were identified among which 30 or $7.8 \%$ were selected, while in the stroke group fewer clusters totalling 334 were identified, yet more of them 41 in total or $12.3 \%$ were selected. This represents a considerable increase of $(12.3-7.8) / 7.8=57.7 \%$ in the number of significant clusters observed in the stroke group, and this increase seems to appear in large part as anticorrelated BOLD response signals. The final two result sections, which directly quantify the space-time structure of the selected clusters and display the corresponding neuroimages, demonstrate that these results inferred by the model are also empirically appreciable.

\subsubsection{Space-time structure of selected clusters}

The space-time parameterisation of contiguity (definition 6, page 89) and causal crosscorrelation (definition 7, page 92) described in this thesis were applied to all selected clusters to examine whether a difference in space-time structure is appreciable. Figure 8.4 shows the space-time structure of the selected clusters across both groups. In figure 8.4a, the contiguity function from all selected clusters for each group were averaged and plotted 
along with the standard error of the mean. The typical function shape, rising sharply from the right and reaching a peak contiguity before falling, is clearly shown for both groups. Interestingly, both groups reach their contiguity maximum for the same threshold value of $r_{c} \approx 0.8$. This suggests that the SNR is comparable between the groups. The contrast between the groups lies in the mean contiguity value of the selected cluster maps. Our data shows that after stroke, the contiguity of the BOLD response signal is significantly diminished, which results in the occurrence of more sparsely distributed BOLD response patterns in stroke patients.

In figure $8.4 \mathbf{b}$, the delay $d$ that maximises the causal cross-correlation function is plotted along the abscissa, and the corresponding correlation $r_{m}$ to the motor response signal is plotted along the ordinate. Because the delay is poorly sampled in blocks of 2 seconds (corresponding to the MRI $T_{R}$ parameter), we jittered these data with uniform random noise over $[-1,1]$ seconds so that the individual points can be seen. As expected from a normal BOLD response, the majority of the selected clusters from the normal group appear in the top-left corner of the graph, corresponding to a positive correlation with the motor signal that is delayed by 2 seconds (c.f. signal 1 in figure 4.3, page 57). In contrast, the stroke group exhibit many anticorrelated clusters with short delays of 1 second, and also positively correlated clusters with long delays of roughly 12 seconds. Furthermore, the stroke group clusters appearing in the expected top-left region of the graph are on average less correlated than those in the normal group. These data suggest that after stroke, a wider variety of response signals being early/anticorrelated or late/correlated appear and on average have a diminished correlation to the motor signal.

Interestingly, several unexpected outliers in the normal group appear to be weakly anticorrelated and delayed by over 6 seconds. We found that all but two of these outliers vanished after adjusting the probability threshold from $p<0.05$ to $p<0.025$, while the positively correlated clusters remained. This suggests that the vanished clusters were marginally selected as false positives. However, two of these clusters, with a 6 -second delay 
and $r_{m}$ of -0.15 and -0.24 , remain and are just as significant as the positively correlated clusters from the normal group. These clusters were identified from the same data set acquired during the first session of normal subject 5 . This is an unusual occurrence that demonstrates either a physiologically significant effect, or a flaw of the Bayesian hierarchical model that must be acknowledged. Therefore, we decided to focus on this case in an attempt to resolve this confound.

To determine why these outliers were selected, we examined the global signal in our normal group data and found that $\boldsymbol{\alpha}$ is normally distributed with mean 0.019 and SD $0.076(n=14)$. However, we found that the session producing these significant outliers has an abnormally high global signal correlation of 0.20 ; in fact, it is the highest observed value in either group. This implies that a large proportion of voxels throughout this subject's brain were correlated with the motor signal; therefore, slightly anticorrelated clusters can potentially stand out from the whole. We ruled out the possibility of stimulusrelated head movements in this session, which suggests that perhaps a stimulus-related and distributed vascular response actually took place in this subject. In addition, the significantly anticorrelated cluster maps are highly contiguous and appear in the basal ganglia and the bilateral premotor cortex, respectively. These regions are well-known to be involved in the performance of motor tasks and, hence, appear to be genuine responses. All considered, there is reason to believe that this anomaly is physiologically significant, since it is known that functional hyperemia is a spatially localised mechanism that does not cause a cerebrum-wide BOLD response signal. Indeed, localised BOLD responses are precisely what we observed in the remainder of our normal group data. Nonetheless, we ought to remain sceptical of the significance of this anomaly, which did not reappear in the second session of normal 5 or elsewhere. Therefore, we recommend that the parameter $\boldsymbol{\alpha}$ be carefully examined for unusually large correlations during analysis because these extreme cases may not be well represented by the proposed Bayesian model. 


\subsubsection{Identified sensorimotor-related brain regions}

Figure 8.5 illustrates the observed BOLD response pattern from the normal group. The selected clusters from all 14 sessions are summarised as an aggregate functional map overlaid on a coregistered transverse structural image shown in radiological convention. The voxels in the selected cluster maps are superimposed, and the colour scale indicates the number of sessions in which the voxels are significant. Because both hands were tested in these tasks, we reflected the functional data acquired during right-handed tasks across the midline so that the expected sensorimotor-related regions can be compared together. Hence, the expected contralateral responses should appear in the right cerebral hemisphere.

Indeed, the expected SMC response is clearly seen in all sessions acquired from the normal group. This demonstrates that the experimental apparatus, data acquisition, and analytical methods proposed here systematically reproduce the BOLD signal response to an event-related motor paradigm. Specifically, the M1 and primary somatosensory areas, bordering the central sulcus contralateral to the moving hand, corresponds in all subjects with a timely and localised BOLD response signal. The secondary cortical areas known to be involved in hand motor tasks corresponded with a second timely and focused BOLD signal in most subjects. These cortical areas are the SMA, located on the dorsomedial cortical surface anterior to $\mathrm{M} 1$, and the premotor and posterior parietal areas ipsilateral to the moving hand. In addition, the putamen and thalamus also corresponded with a BOLD response signal. These deep brain centres appear in the second row from the top of this figure, periventricular to the anterior horns.

Therefore, in agreement with the sensorimotor system literature reviewed in chapter 2 (c.f. figure 2.2, page 25), the reproducible involvement of these specific regions seen here supports the current evidence that multiple sensorimotor subcircuits interact across distributed networks during the performance of somatosensory function. Two unexpected cortical regions, the superior temporal gyrus and the insula, also corresponded with a bilat- 


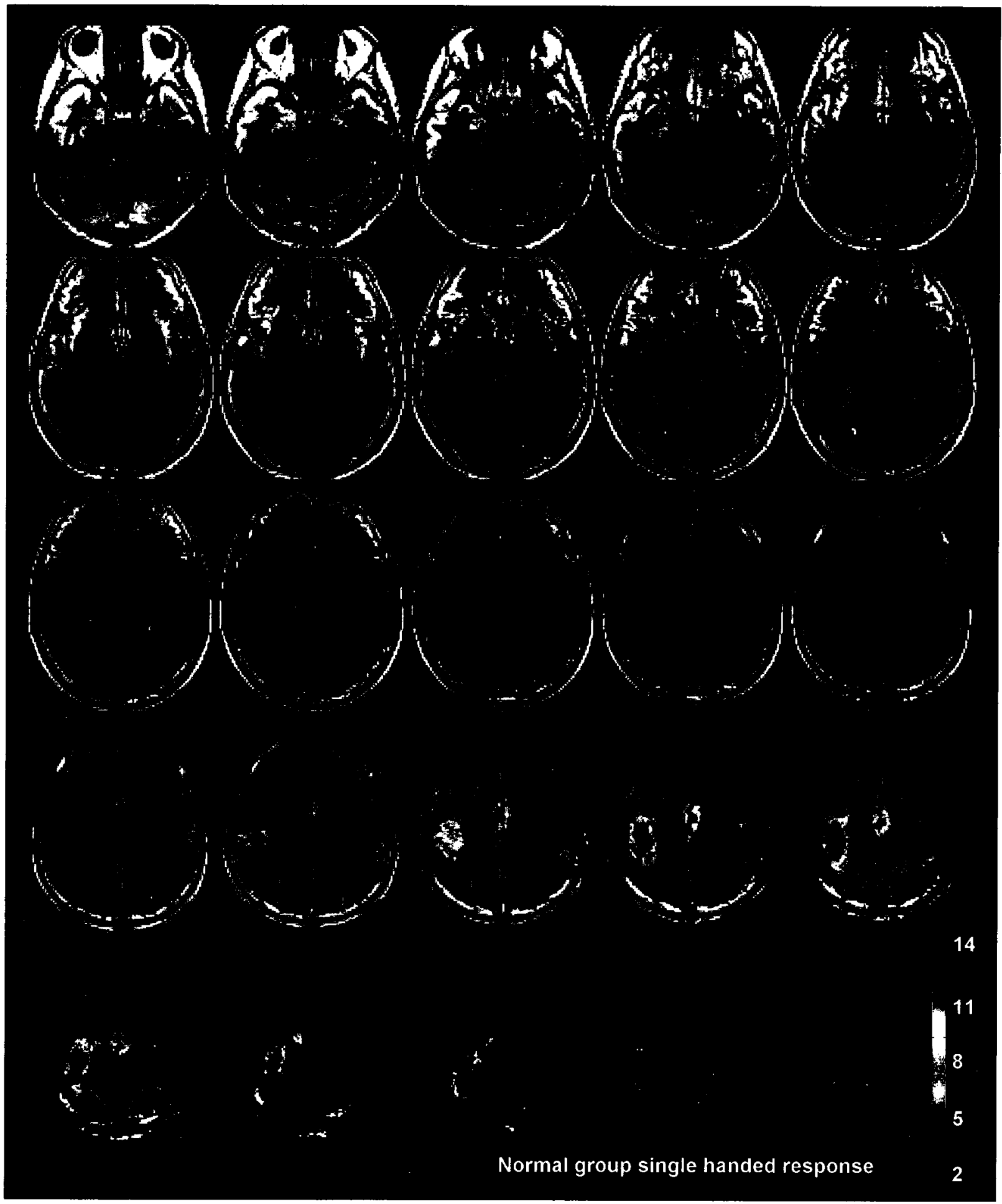

Figure 8.5: BOLD response signal from the normal group performing the eventrelated motor task with either hand shown in radiological convention on a coregistered transverse structural image from a normal subject. Neuroimage data from righthanded sessions are reflected across the midline. Voxels in the selected cluster maps from all 14 sessions are superimposed, and the colour scale indicates the number of sessions in which the voxels are significant. 
eral BOLD response signal in several subjects. These regions, however, are not considered central to the functional anatomy of the sensorimotor system. One plausible explanation for these observed BOLD responses might follow from the fact that the SMC and these regions are located on the same cerebrovascular territory, which is primarily perfused by the MCA (c.f. figure 2.4, page 29). Consequently, one could speculate that the blood flow in these regions is affected to some extent by the retrograde vasodilation taking place during functional hyperemia in the SMC. The remaining infrequently observed regions visible within the cerebellum and the prefrontal cortex are less of interest in this paradigm because they operate at abstract levels and so are involved in many faculties.

The characterisation of the space-time structure of the stroke group data indicated a marked deterioration in the contiguity and correlation of the BOLD response signal in this event-related paradigm. These effects are also clearly shown in the aggregate results in figures 8.6 and 8.7, which show the BOLD signal clusters selected from the stroke patients performing the motor task using either their ipsilesional hand, or their contralesional hand, respectively. In these figures, we reflected the functional data acquired from patient 2 across the midline so that the expected sensorimotor-related regions from ipsilesional and contralesional hands can be compared together. The arrows in the stroke group figures indicate the slice and hemisphere where the SMC response is expected according to the normal group results.

These results provide direct evidence that although the patients were performing the task, the expected functional hyperemia is largely absent. Instead, a widespread and poorly correlated neurovascular response takes place inconsistently across the stroke group. Indeed, the infrequent and patchy BOLD response patterns covering most of the cortical surface of these stroke patients is strikingly different from the reproducible and highly focused BOLD response in the normal subjects. There are two intriguing potential explanations behind this phenomenon: i) neuronal plasticity is taking place, and the observed patient-specific sparse cortical centres are acting in concert to compensate for the 


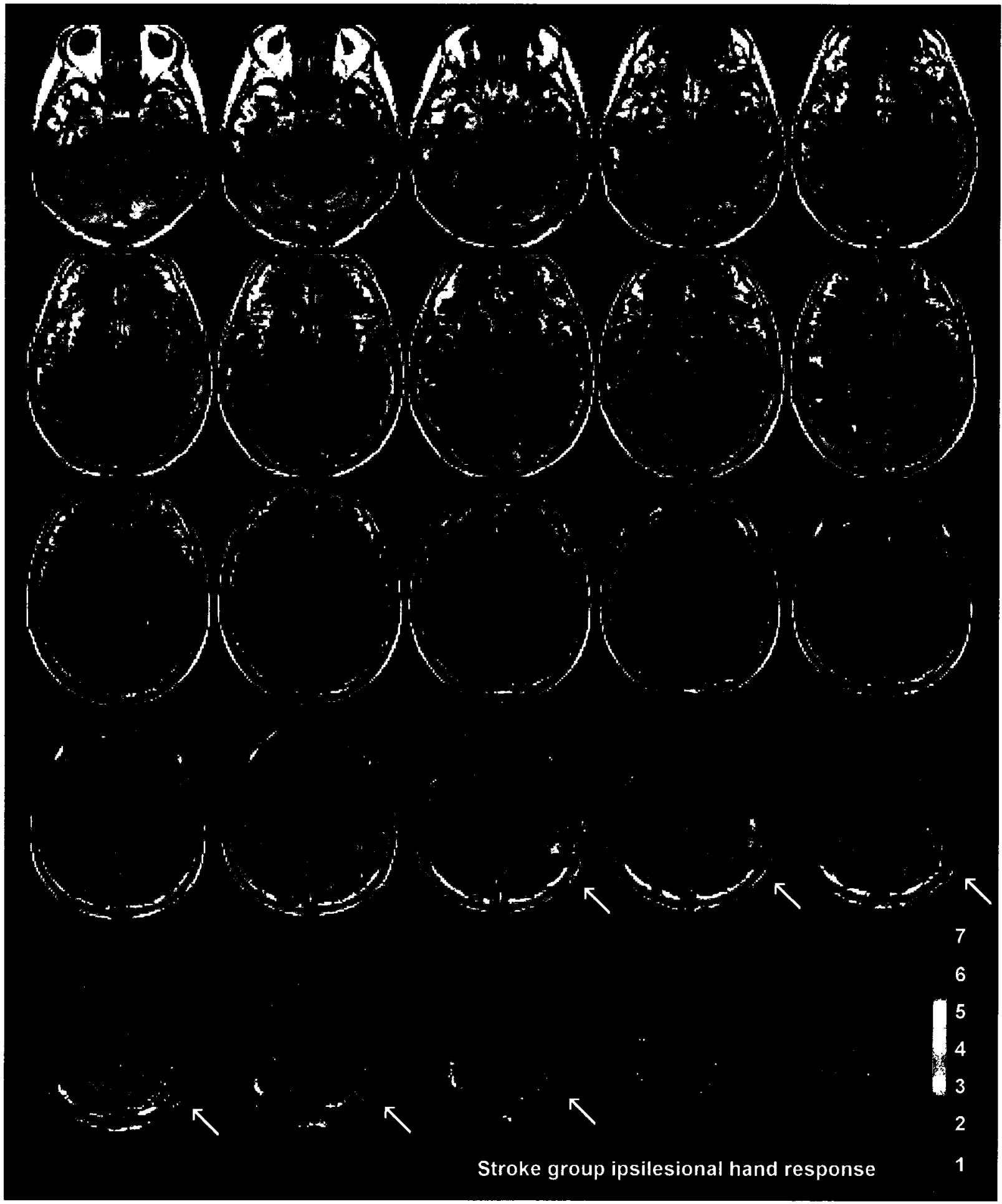

Figure 8.6: BOLD response signal from the stroke group performing the eventrelated motor task with the ipsilesional hand. The overlay is shown in radiological convention on a coregistered transverse structural image from a normal subject. Neuroimage data from left-handed sessions are reflected across the midline. Voxels in the selected cluster maps from all 7 sessions are superimposed, and the colour scale indicates the number of sessions in which the voxels were significant. Arrows indicate slice and hemisphere where the SMC response is expected according to normal group results. 

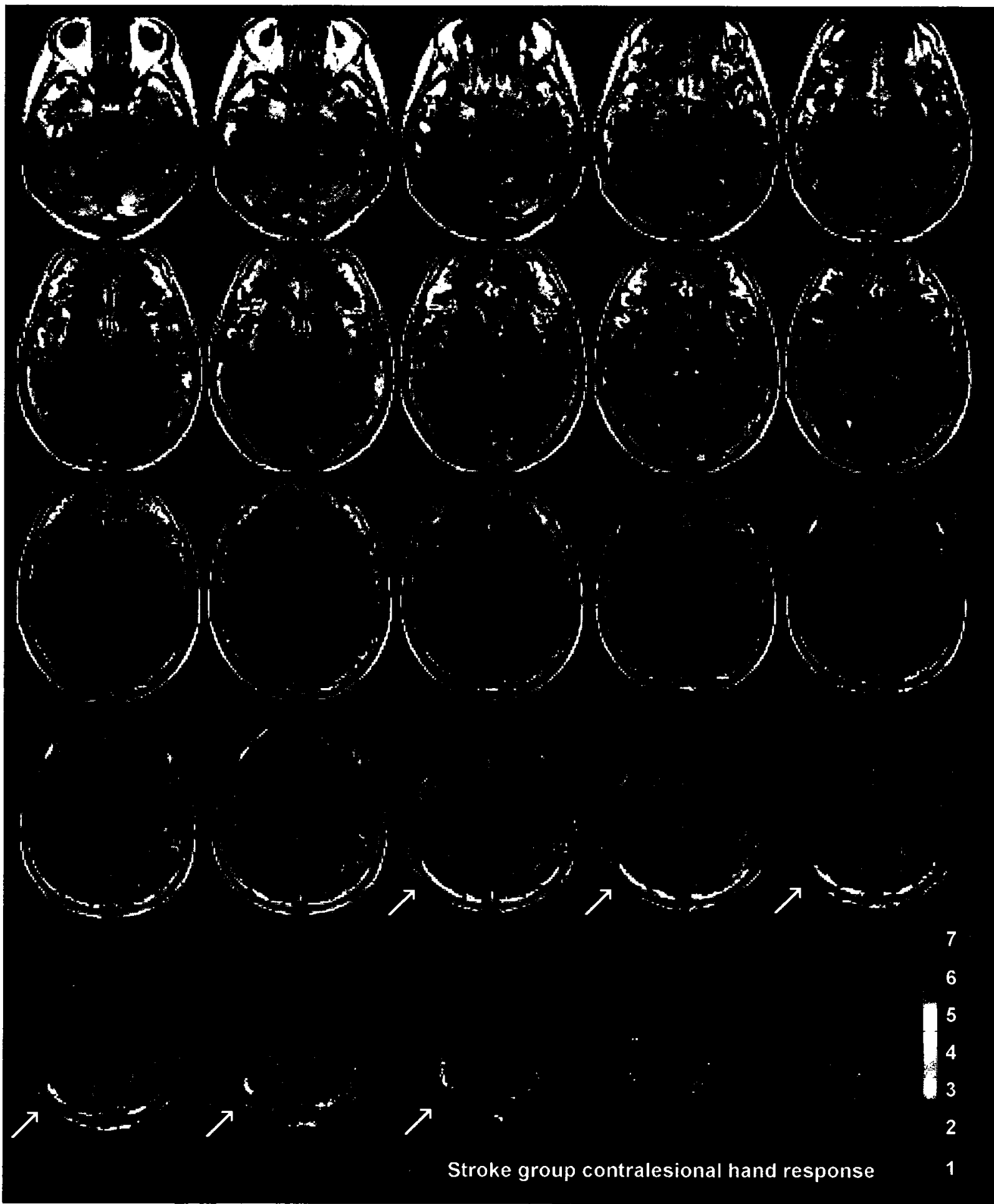

Figure 8.7: BOLD response signal from the stroke group performing the eventrelated motor task with the contralesional hand. The overlay is shown in radiological convention on a coregistered transverse structural image from a normal subject. Neuroimage data from right-handed sessions are reflected across the midline. Voxels in the selected cluster maps from all 7 sessions are superimposed, and the colour scale indicates the number of sessions in which the voxels were significant. Arrows indicate slice and hemisphere where the SMC response is expected according to normal group results. 
damaged sensorimotor system, or ii) the cerebrovascular system has been compromised by stroke, and the patient-specific BOLD signals appearing sporadically do not correspond to colocalised neuronal activity. Of course, isolating the factors that lead to our observations would require a larger study that also accounts for the confounding factors between the groups (e.g., age), as well as, the co-morbidity factors associated with stroke (e.g., hypertension, smoking, diabetes). However, based on our current results and our review of neurophysiology and the cerebrovascular system in the first two chapters, we may speculate that neurovascular dysfunction is a more plausible cause than neuronal plasticity to explain the sparse and inconsistent BOLD response patterns we have observed. Consider the following arguments. First, the cytoarchitectural organisation of the human brain is remarkably consistent across individuals; e.g., consider the validity of Brodmann's areas, and the consistent BOLD response pattern seen in our normal group data. Hence, if neuronal plasticity was taking place, we would expect to see BOLD responses appearing somewhat focused in the motor-related areas, yet this is clearly not the case in our data. Second, Rossini et al. (2003) have failed to detect an SMC-localised functional hyperemia in half of their unilateral stroke patients performing motor tasks. However, they detected in all patients increased SMC-localised neuronal activity. Indeed, functional hyperemia is a complex phenomenon that relies on a multitude of cells and molecular signalling mechanisms to provide the neurovascular coupling between neuronal activity and the cerebral circulation. Consequently, pathophysiology extending beyond the ischemic core and penumbra could potentially render dysfunctional the neurovascular unit throughout the cerebrovascular system.

In addition to this general lack of a consistent hemodynamic response, more subtle differences are appreciable between the results obtained from the ipsilesional and contralesional hands. Specifically, the ipsilesional data continue to show in most sessions a BOLD response in the expected SMC (indicated by the arrows in figure 8.6) and the SMA. This result is expected based on the literature presented in chapter 2, and on the knowl- 
edge that the SMC contralateral to this hand was not ischemic. However, in the data acquired during contralesional hand movement, this expected response is almost entirely absent (indicated by the arrows in figure 8.7). These contralesional data instead reveal in most sessions a small, unexpected BOLD response in the hemisphere ipsilateral to the moving hand (i.e., the hemisphere which was not ischemic) predominantly in the primary somatosensory area and the posterior parietal area. Although the sensorimotor system is known to operate to some extent across hemispheres, as suggested by the normal group result, little is known on whether such a bilateral interaction is sufficient for enabling centres in one intact hemisphere to compensate for deficiencies incurred in the other hemisphere. While these results suggest the presence of a neural-driven BOLD response ipsilateral to the paretic hand in the majority of the stroke group data, it remains to be seen whether this response is reproducible in future studies of unilateral stroke with motor deficit.

Individual neuroimage analysis results are now shown in order to demonstrate the performance of the proposed model as well as the differences between groups. Figure 8.8 presents a summary of the results from the second session of normal subject 5 , and figure 8.9 similarly shows the results from stroke patient 1 at 3 months post-stroke. At the top of each figure, the Bayesian hierarchical model simulates the distribution of voxel time sequence correlations with the motor response signal $\left(r_{m}\right)$ and shows their boxplots for the global signal $\boldsymbol{\alpha}$ and each cluster $\boldsymbol{\beta}_{i}$, for $i=1, \ldots, K$. For visual comparison, the $95 \%$ probability interval of $\boldsymbol{\alpha}$ is bounded between the two horizontal grey lines. The selected clusters (marked by ${ }^{*}$ ) are considered significantly different from $\boldsymbol{\alpha}$ if their Bayes' error is $p<0.05$. At most three of the selected clusters are presented in each figure in order of decreasing Bayes' error. For each cluster, starting from left of the figure, a computer rendition of the member voxel position (shown in green) relative to the brain (delineated in grey) are shown from sagittal (top left), coronal (top right), and transverse (bottom) views. A corresponding coregistered anatomical overlay is shown in radiological convention containing the voxel with maximum centroid time sequence correlation $r\left(x, v_{i}\right)$ whose value 


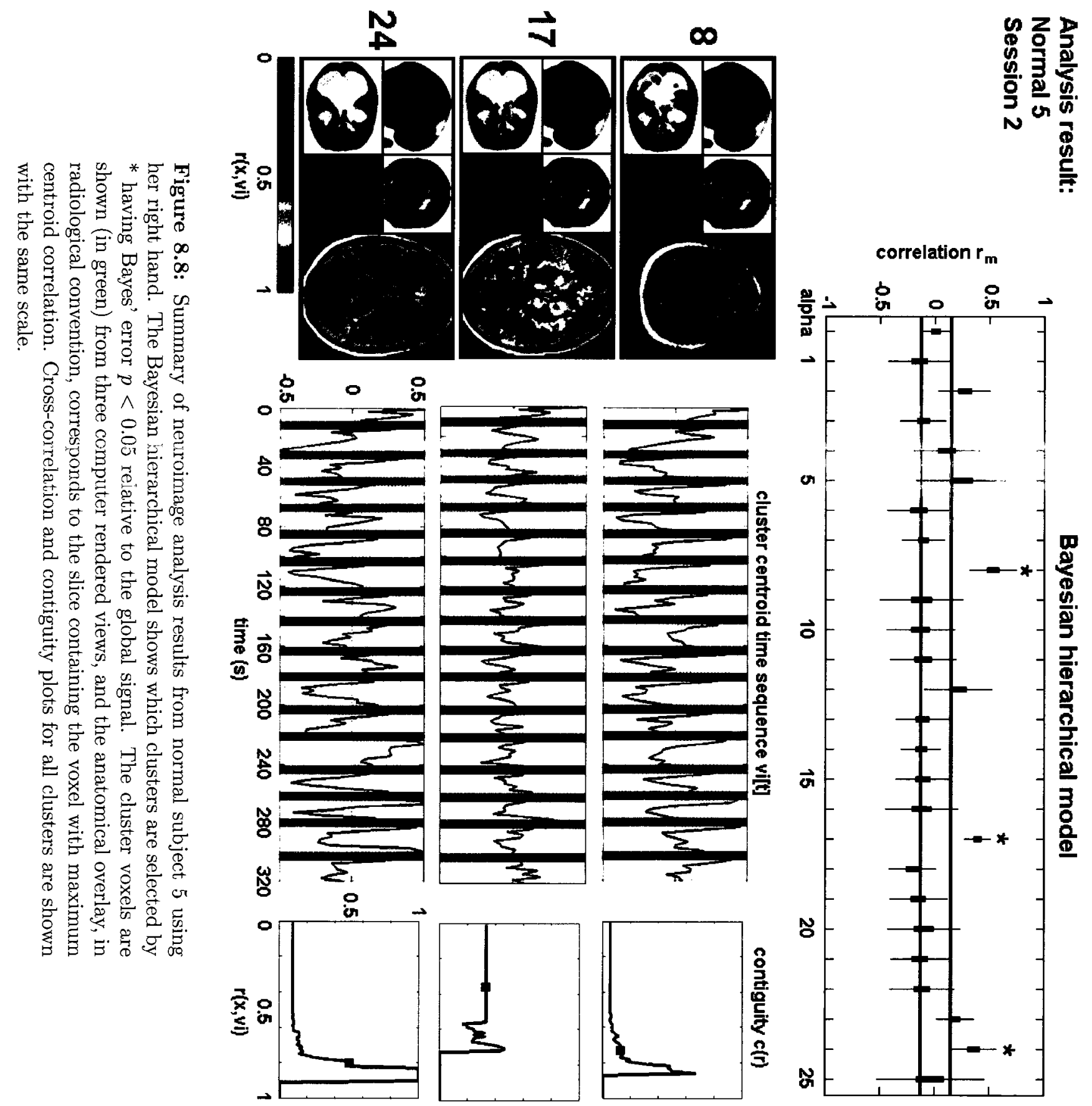




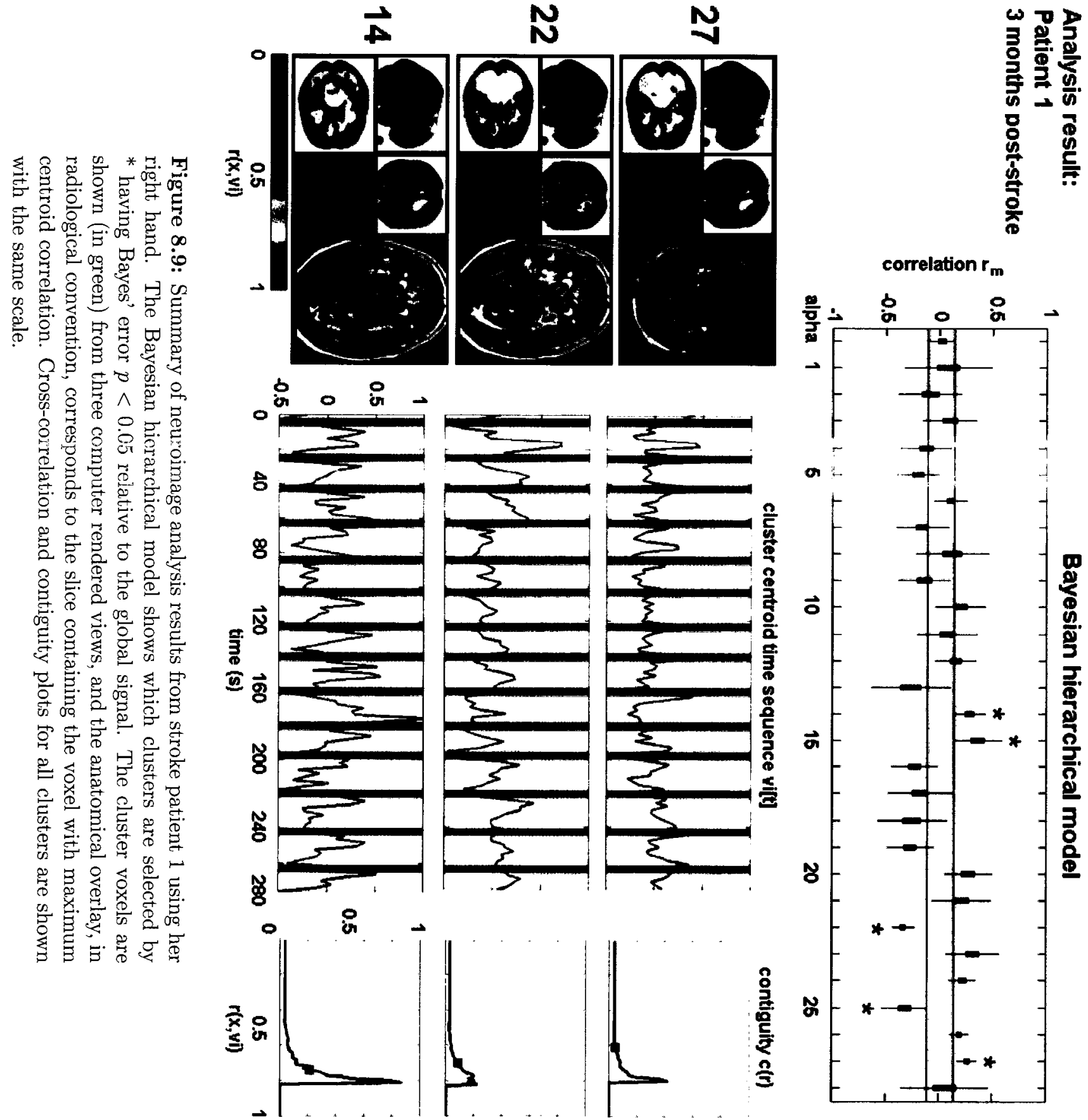


is indicated by the colour scale. Finally, the graphs for the cluster centroid time sequence $v_{i}[t]$ in comparison to the motor responses (grey bars), and the cluster contiguity function $c(r)$ are shown using the same scale within each figure.

In both cases shown here, the subjects used their right hand to perform the task, and their left-hemisphere SMC was identified as cluster 8 in the normal subject and cluster 27 in the stroke patient. In addition, regions within the putamen and thalamus were identified as shown in clusters 17 in the normal subject and cluster 22 in the stroke subject. Notice how in the normal case the centroid is positively correlated, while in the stroke case it is anticorrelated. The emergence of anticorrelated clusters is typically demonstrated here when comparing the boxplots between these subjects shown in the Bayesian hierarchical model. The third unexpected clusters shown in these subjects are marginally significant and lack the same synchronisation of the previous two clusters yet reached a significant threshold nonetheless. While it would be easy to eliminate these clusters by choosing a slightly smaller $p$, it is perhaps better to admit these clusters, verify their location, and keep track of how often they appear across subjects before interpreting their significance with respect to the task. Finally, these subjects demonstrate how the cluster contiguity is typically diminished in the stroke group as can be seen in the cluster renditions and their contiguity functions.

\subsection{Discussion}

This chapter applied the analytical methods developed throughout this thesis as a whole to i) study the neuroimage data acquired from our normal and ischemic stroke groups, and ii) to interpret the results in corroboration with the evidence reported in the neurological and cerebrovascular literature. The sample sizes of the groups participating in this research are small in relation to those required to make inference at the population level. Hence, our clinical interpretation of the results are limited. However, the data acquired from 
these groups demonstrate the performance of the novel analytical methods proposed in this thesis - the principal aim of this research - and also provide supporting evidence of the reproducibility of the BOLD response to event-related motor tasks in the normal subject population as defined here.

The results presented in this chapter do indeed show that the proposed analytical method is capable of characterising and distinguishing between the space-time structure of the identified response patterns in normal subjects and ischemic stroke patients. Specifically, in the stroke group, the number of responding brain regions identified increases and appear in normally unresponsive areas throughout the cerebrum relative to the normal group. Moreover, these regions possess a diminished spatial contiguity, and a diminished correlation to the motor responses that are either extensively delayed, or early and anticorrelated.

The stroke patients who participated in our study illustrate the large variability in how cerebrovascular disease can affect an individual. While our aim was to recruit a sample of first-ever stroke patients with motor deficits that represent this population as closely as possible, the size of the group included in this research turned out to be rather small. Consequently, we chose to use this limited data set in the best way possible to provide the aggregate results shown here. Specifically, we decided to pool our stroke data in two groups: sessions using the ipsilesional hand, and those using the contralesional hand. To do so, we essentially did not account for the potential effects introduced by hemispheric asymmetry by reflecting some of our neuroimage data across the cerebral midline so that comparisons could be made. Secondly, although three of our patients returned for a second visit roughly three months post-stroke, we chose in this research to pool all of our data into a single time point for analysis. Essentially, we thought that the group was too small to subdivide any further and that, instead, a single aggregate group would be more effective to demonstrate the proposed analytical methodology and the most prominent differences between the normal and stroke groups. We also recognise 
that our study is inadequate for a compelling physiological interpretation of our results. A larger study is required that accounts for the confounding factors between the groups (e.g., age), as well as, the co-morbidity factors associated with stroke (e.g., hypertension, smoking, diabetes) in order to identify how these factors influence our results.

Nonetheless, we can speculate based on the literature reviewed in this thesis, that the results from our stroke group are predominantly affected by neurovascular dysfunction. According to the current understanding of cerebrovascular autoregulation and functional hyperemia (Attwell \& Iadecola, 2002; D'Esposito et al., 2003; Girouard \& Iadecola, 2006; Hamel, 2006), it is plausible that the dispersed responses appearing throughout the cerebral cortex of the stroke patients are the result of a retrograde vasodilation, possibly extending back to the larger arteries, whose increase in perfusion is inadequately regulated by the microvasculature throughout most of the brain. To verify this hypothesis, it would be necessary to recruit a cohort of ischemic stroke patients with motor deficits, that are age-matched with a cohort of normal subjects, and monitor their recovery over time using perhaps a similar event-related motor paradigm in order to determine whether good motor recovery correlates with the disappearance of the spurious BOLD response patterns leaving only the expected SMC-related and/or potentially novel cortical regions colocalised with a timely and focused BOLD response signal. 


\section{Chapter 9}

\section{Conclusion}

\subsection{Introduction}

This chapter concludes the thesis by summarising the salient points of the research described herein whose principal objective was to develop novel analytical methods for functional neuroimage data. The motivation for developing new analysis methods stems from our ever growing awareness of the complexity of the human brain. It seems natural in science that as our knowledge base grows, new questions come forward, which in turn depend on new methods to obtain answers.

To write this thesis, it was necessary to draw from the many disciplines that form the consilience of neuroscience in order to appreciate this remarkable biological system. This interdisciplinary study has provided us with the appropriate vantage point to identify the neurological questions that can be addressed with functional neuroimaging. Indeed, the latest knowledge on the profound cerebrovascular changes that follow stroke has motivated this proposal of exploratory pattern recognition for functional neuroimage data. Furthermore, the neurophysiological literature synthesised here was also fundamental in experimentally observing these changes and comparing their space-time characteristics. Hopefully, the consolidation of the interdisciplinary material presented here goes beyond 
the thesis motivations by providing a broader perspective of neuroscience that is needed to ponder the fundamental questions on the subject.

\subsection{Summary and critique of the proposed methods}

A critique of the proposed methods is provided here, which summarises the limiting assumptions, advantages, and issues discussed throughout the thesis. Potential avenues for future work are also mentioned here.

The experimental design used in this research was developed particularly to characterise the sensorimotor system using BOLD fMRI data acquired from healthy volunteers and ischemic stroke patients with motor impairments. Consequently, its ability to characterise impairments in other systems was not assessed and should be limited to studies focused on the topics described here. Our experimental protocol proved to be flexible enough to account for the wide range of impairments and clinical needs of the stroke patients in our study. We also found that the event-related motor paradigm was effective in engaging all participants, and succeeded in systematically reproducing functional hyperemia in our normal group. The major issues with our approach are technological in nature. MRI is very sensitive to head movements and generates prominent artefacts in our functional neuroimage data. We found these difficult to avoid in our stroke group due to their involuntary motion. MRI is not readily compatible with other electromagnetic instruments, which makes the simultaneous imaging of both neural and vascular components difficult. This limited our comparisons to muscular responses in the hands instead of direct measures of neural activity.

The preliminary analysis of our BOLD fMRI data was successful in measuring the degree of correlation present within each session. This analysis was instrumental in i) selecting the MRI RF coil settings with smallest correlation values, and ii) modelling the noise process for BOLD fMRI data simulation. An important direction for future research 
is to develop statistical methods to extract independent samples from highly correlated neuroimage data. For example, one approach that shows promise is the application of autoregressive models.

When working with the original correlated data, this thesis proposes a novel time series bootstrap algorithm for neuroimage session simulations. Although this approach has the advantage of generating many neuroimage session simulations with realistic anatomical and functional traits, a limitation is that the original neuroimage acquisitions require additional techniques to obtain consistent slice positioning so that each simulation retains a realistic anatomy. A potential future project is to develop and test consistent slice positioning techniques to attempt the generation of large scale bootstrap simulations from null fMRI data.

The fuzzy $k$-means pattern recognition algorithm used to partition each neuroimage session was successful in distinguishing between distinct BOLD signal responses. Our approach to determine the optimal clustering number $k$ relied upon previous work based on a heuristic technique. Although this algorithm is suitable for this problem due to its ability to identify the presence of compact well-separated clusters, a direct link to a probabilistic framework has not been found, which would eliminate the need for the currently-used heuristics. Notably, the notion of information entropy may be helpful in making this link. Consequently, we addressed this problem by proposing a novel computer-intensive technique that could lead to lower-bound probability estimates for partition entropy of neuroimage data sets. The major limitation of this approach is the computational resources required, preventing us from showing more than a preliminary result from a small data set. For future work, it would be interesting to perform these entropy estimates on a large data set to determine if these entropy estimates could lead to a probability-based fuzzy $k$-means clustering method.

A novel method to characterise the space-time structure of neuroimage data is proposed in this thesis. We applied these characteristics to neuroimage data and demon- 
strated their usefulness as selection criteria for the identification of stimulus-related voxel clusters. However, the criteria used here should be limited to the study of the BOLD signal induced by functional hyperemia, since they were designed based on the known features of this response. Caution is advised, since these features may not apply in other neuroimage modalities or for the use with different experimental paradigms.

A Bayesian hierarchical model is proposed for the analysis of neuroimage data in this thesis. This idea has also been proposed recently by another research group whose authors are arguably amongst the leading statisticians in Bayesian analysis. We conceived this idea independently and demonstrated that, indeed, this model is suitable to represent the hierarchical nature of voxel time sequence correlations in our neuroimage data. Furthermore, the model also accounts for the potential bias introduced by the global signal present throughout the data. It was advantageous to implement the model using the Markov chain Monte Carlo simulation technique so that the model's probability densities can be modified without the need of calculating analytical expressions for joint probability models. For example, a project for future work would be to apply this model with a more general parametric form of the global signal density function to account for non-Gaussian effects, e.g., the skews or spikes occasionally encountered in some of our neuroimage sessions.

\subsection{Summary of the neuroimage stroke trial results}

The research undertaken in this thesis is dedicated to the advancement of our understanding of the regulatory mechanisms of the cerebral circulation and its dysfunction in disease. This thesis presents neuroimage results from relatively small population samples from healthy volunteers and ischemic stroke patients. The proposed method was successful in differentiating between these populations.

Throughout our neuroimage experiments involving healthy volunteers, our methods 
were successful at corroborating and reproducing the expected functional hyperemia related to sensorimotor function. Namely, the consistent appearance of localised and timely functional hyperemia in the sensorimotor cortex contralateral to the moving hand and in the supplementary motor areas. Secondary responses that are also associated with the sensorimotor system were also identified in the premotor and posterior parietal areas ipsilateral to the moving hand. Occasionally, responses within the basal ganglia and thalamus were also encountered. As discussed throughout the thesis, these sensorimotor centres are known to play key roles in visual feedback controlled motor tasks and have been observed in other neuroimaging studies similar to ours.

Secondly, we applied this method to provide preliminary results on how ischemic stroke may affect the sensorimotor system. In contrast to the results from the normal subjects, the neuroimage experiments involving the stroke patients produced strikingly different responses. In these patients, a two-fold increase in the number of responding brain regions was observed. These regions had a significantly diminished spatial contiguity. Specifically, these response patterns were not confined to the expected sensorimotor centres but rather appeared throughout most of the cerebral grey matter. Furthermore, the timely and consistent cross-correlation structure of the normal group responses appears to have deteriorated in the stroke group data. Our results show that this temporal relationship with the motor signal was substantially more variable than in the normal group due to the unexpected appearance of i) early and anti-correlated responses, and ii) late and positively correlated responses.

It is most likely that these widely dispersed response signals appearing throughout the cerebral cortex of the stroke patients are no longer coupled to colocalised neural activity. Alternative interpretations based on cortical plasticity, i.e., the recruitment of unexpected areas of cortex to compensate for the infarcted tissue, are not likely to explain our results because the observed responses are not contiguous, timely, nor consistent within the stroke group. According to this premise, our results suggest that the mechanisms of 
cerebral autoregulation and functional hyperemia can remain compromised in the months following ischemic stroke.

\subsection{General conclusion and future directions}

In conclusion, this thesis provides direct evidence supporting the application of exploratory pattern recognition and the Bayesian analysis of neuroimage data acquired during the study of ischemic stroke and its impact on the cerebrovascular system. The benefit of using such algorithms to study the complex regulatory mechanisms and their interaction with the nervous system in the wake of cerebrovascular disease may be unprecedented. Therefore, it is recommended that the analytical methods proposed here be extended into a longitudinal study of recovering ischemic stroke patients with motor deficits. Monitoring the spacetime structure of the neurovascular response to motor tasks during recovery would provide key information on our ability to directly assess the efficacy of stroke rehabilitation and help validate future programmes. 


\section{Bibliography}

Abbott, A. (2009). Opening up brain surgery, in Nature News. Nature, 461, 866-868.

Ashburner, J. (2008). SPM5 Manual: The FIL Methods Group. Functional Imaging Laboratory, Wellcome Trust Centre for Neuroimaging.

Attwell, D. \& Iadecola, C. (2002). The neural basis of functional brain imaging signals. Trends Neurosci., 25, 621-625.

Badre, D., Hoffman, J., Cooney, J.W. \& D'Esposito, M. (2009). Hierarchical cognitive control deficits following damage to the human frontal lobe. Nat. Neurosci., 12, 515522 .

Ball, G.H. \& Hall, D.J. (1967). ISODATA, an iterative method of multivariate analysis and pattern classification. Behavioural Science, 12, 153-155.

Barr, M.L. (1974). The Human Nervous System: an Anatomical Viewpoint. Harper \& Row, Hagerstown, USA.

Bezdek, J.C. (1981). Pattern Recognition with Fuzzy Objective Function Algorithms. Plenum, New York, USA.

Bezdek, J.C. \& Pal, S.K. (1992). Fuzzy Models for Pattern Recognition: Methods That Search for Structure in Data. IEEE, New York, USA.

Bouguessa, M., Wang, S. \& Sun, H. (2006). An objective approach to cluster validation. Patt. Recog. Lett., 27, 1419. 
Boynton, G.M., Engel, S.A., Glover, G.H. \& Heeger, D.J. (1996). Linear systems analysis of functional magnetic resonance imaging in human V1. J. Neurosci., 16, 4207-4221.

Breasted, J.H. (1930). The Edwin Smith Surgical Papyrus. The University of Chicago Press, Chicago, USA.

Bundo, M., Inao, S., Nakamura, A., Kato, T., Ito, K., Tadokoro, M., Kabeya, R., Sugimoto, T., Kajita, Y. \& Yoshida, J. (2002). Changes of neural activity correlate with the severity of cortical ischemia in patients with unilateral major cerebral artery occlusion. Stroke, 33, 61-66.

Burke, M. \& Bührle, C. (2006). Bold response during uncoupling of neuronal activity and CBF. NeuroImage, 32, 1-8.

Buxton, R.B., Uludă̆, K., Dubowitz, D.J. \& Liu, T.T. (2004). Modeling the hemodynamic response to brain activation. NeuroImage, 23, S220-S233.

Cardenas-Blanco, A., Tejos, C., Irarrazaval, P. \& Cameron, I. (2008). Noise in magnitude resonance images. Magn. Reson. Part A, 32, 409.

Carusone, L.M., Srinivasan, J., Gitelman, D.R., Mesulam, M.M. \& Parrish, T.B. (2002). Hemodynamic response changes in cerebrovascular disease: implications for functional MR imaging. Am. J. Neuroradiol., 23, 1222-1228.

de Pasquale, F., Gratta, C.D. \& Romani, G.L. (2008). Empirical Markov chain Monte Carlo Bayesian analysis of fMRI data. NeuroImage, 42, 99-111.

Desjardins, A.E., Kiehl, K.A. \& Liddle, P.F. (2001). Removal of confounding effects of global signal in functional MRI analyses. NeuroImage, 13, 751-758.

D'Esposito, M., Deouell, L.Y. \& Gazzaley, A. (2003). Alterations in the BOLD fMRI signal with ageing and disease: a challenge for neuroimaging. Nature Rev. Neurosci., $\mathbf{4}$, $863-872$. 
Devroye, L., Györfi, L. \& Lugosi, G., eds. (1996). A Probabilistic Theory of Pattern Recognition. Springer-Verlag, Berlin, Germany.

Doyle, K.P., Simon, R.P. \& Stenzel-Poore, M.P. (2008). Mechanisms of ischemic brain damage. Neuropharmacology, 55, 310-318.

Duda, R.O. \& Hart, P.E. (1972). Pattern Classification and Scene Analysis. Academic Press, New York, USA.

Duda, R.O., Hart, P.E. \& Stork, D.G. (2001). Pattern Classification. Wiley-Interscience, New York, USA, 2nd edn.

Dunn, J. (1973). A fuzzy relative of the ISODATA process and its use in detecting compact well-separated clusters. J. Cybern, 3, 32-57.

Efron, B. \& Tibshirani, R. (1991). Statistical data analysis in the computer age. Science, 253, 390-395.

Esposito, F., Scarabino, T., Hyvarinen, A., Himberg, J., Formisano, E., Comani, S., Tedeschi, G., Goebel, R., Seifritz, E. \& Salle, F.D. (2005). Independent component analysis of fMRI group studies by self-organising clustering. NeuroImage, 25, 193-205.

Feng, J., ed. (2004). Computational Neuroscience: a Comprehensive Approach. Chapman \& Hall/CRC, Boca Raton, USA.

Finger, S. (1994). Origins of Neuroscience: a History of Explorations into Brain Function. Oxford University Press, New York, USA.

Fox, P.T. \& Raichle, M.E. (1986). Focal physiological uncoupling of cerebral blood flow and oxidative metabolism during somatosensory stimulation in human subjects. Proc. Natl. Acad. Sci., 83, 1140-1144.

Fox, P.T., Raichle, M.E., Mintun, M.A. \& Dence, C. (1988). Nonoxidative glucose consumption during focal physiological neural activity. Science, 241, 462-464. 
Friston, K.J., Holmes, A.P., Worsley, K.J., Poline, J.P., Frith, C.D. \& Frackowiak, R.S.J. (1995). Statistical parametric maps in functional imaging: a general linear approach. Hum. Brain Mapp., 2, 189-210.

Friston, K.J., Fletcher, P., Josephs, O., Holmes, A., Rugg, M.D. \& Turner, R. (1998). Event-related fMRI: characterizing differential responses. NeuroImage, 7, 30-40.

Garey, L.J., ed. (1994). Brodmann's 'Localisation in the Cerebral Cortex'. Smith-Gordon, London, UK, 2nd edn.

Gelman, A., Carlin, J.B., Stern, H.S. \& Rubin, D.B. (2004). Bayesian Data Analysis. Chapman \& Hall/CRC Press, Boca Raton, USA.

Girouard, H. \& Iadecola, C. (2006). Neurovascular coupling in the normal brain and in hypertension, stroke, and alzheimer disease. J. Appl. Physiol., 100, 328-335.

Golay, X., Kollias, S., Stoll, G., Meier, D., Valavanis, A. \& Boesiger, P. (1998). A new correlation-based fuzzy logic clustering algorithm for fMRI. Magn. Reson. Med., 40, 249-260.

Gómez-Laberge, C., Adler, A., Cameron, I., Nguyen, T. \& Hogan, M.J. (2008). Selection criteria for the analysis of data-driven clusters in cerebral fMRI. IEEE Trans. Biomed. Eng., 55, 2372-2380.

Gordon, G.R., Choi, H.B., Rungta, R.L., Ellis-Davies, G.C.R. \& MacVicar, B.A. (2009). Brain metabolism dictates the polarity of astrocyte control over arterioles. Nature, 456, 745-750.

Goutte, C., Toft, P., Rostrup, E., Nielsen, F.A., \& Hansen, L.K. (1999). On clustering fo fMRI time series. NeuroImage, 9, 298-310.

Grey, H. (1977). Anatomy, Descriptive and Surgical. Random House, New York, USA. 
Hamel, E. (2006). Perivascular nerves and the regulation of cerebrovascular tone. J. Appl. Physiol., 100, 1059-1064.

Harrison, L., Penny, W.D. \& Friston, K. (2003). Multivariate autoregressive modeling of fMRI time series. NeuroImage, 19, 1477-1491.

Hathaway, R.J., Bezdek, J.C. \& Huband, J.M. (2006). Scalable visual assessment of cluster tendency for large data sets. Patt. Recog., 39, 1315.

Hochberg, L.R., Serruya, M.D., Friehs, G.M., Mukand, J.A., Saleh, M., Caplan, A.H., Branner, A., Chen, D., Penn, R.D. \& Donoghue, J.P. (2006). Neuronal ensemble control of prosthetic devices by a human with tetraplegia. Nature, 442, 164-171.

Hodgkin, A.L. \& Huxley, A.F. (1952). A quantitiative description of membrane current and its application to conduction and excitation in nerve. J. Physiol., 117, 500-544.

Howell, D.C. (1992). Statistical Methods for Psychology. Duxbury Press, Belmont, USA.

Huettel, S.A., Singerman, J.D. \& McCarthy, G. (2001). The effets of aging upon the hemodynamic response measured by functional MRI. NeuroImage, 13, 161-175.

Iadecola, C. \& Nedergaard, M. (2007). Glial regulation of the cerebral microvasculature. Nat. Neurosci., 10, 1369-1376.

Ito, H., Ibaraki, M., Kanno, I., Fukuda, H. \& Miura, S. (2005). Changes in cerebral blood flow and cerebral oxygen metabolism during neural activation measured by positron emission tomography: comparison with blood oxygenation level-dependent contrast measured by functional magnetic resonance imaging. J. Cereb. Blood Flow Metab., 25, 371-377.

Jarmasz, M. \& Somorjai, R.L. (1998). Time to join! cluster-merging in unsupervised fuzzy clustering for functional MRI data. In Proc. ISMRM 6th Annu. Meeting, 2068, Sydney, Australia. 
Jarmasz, M. \& Somorjai, R.L. (2002). Exploring regions of interest with clustering analysis (EROICA) using a spectral peak statistic for selecting and testing the significance of fMRI activation time series. Artif. Intell. Med., 25, 45-67.

Joyce, K.A. (2008). Magnetic Appeal: MRI and the Myth of Transparency. Cornell University Press, Ithaca, USA.

Kandel, E.R., Schwartz, J.H. \& Jessel, T.M. (2000). Principles of Neural Science. McGraw Hill, New York, USA, 4th edn.

Knešaurek, K. \& Machac, J. (2006). Comparison of ${ }^{18}$ F SPECT with PET in myocardial imaging: a realistic thorax-cardiac phantom study. BMC Nucl. Med., 6.

Kocharyan, A., Fernandes, P., Tong, X.K., Vaucher, E. \& Hamel, E. (2008). Specific subtypes of cortical GABA interneurons contribute to the neurovascular coupling response to basal forebrain stimulation. J. Cereb. Blood Flow Metab., 28, 221-31.

Kolmogorov, A.N. (1957). On the representation of continuous functions of many variables by superpositions of continous functions of one variable and addition. Doklady Akademii Nauk USSR, 14, 953-956.

Kürková, V. (1991). Kolmogorov's theorem is relevant. Neural Comput., 3, 617-622.

Kuschinsky, W. (2000). Regulation of cerebral blood flow. In C.T. Moonen \& P.A. Bandettini, eds., Functional MRI, 15-24, Springer-Verlag, Berlin, Germany.

Kwong, K.K., Belliveau, J.W., Chesler, D.A., Goldberg, I.E., Weisskoff, R.M., Poncelet, B.P., Kennedy, D.N., Hoppel, B.E., Cohen, M.S. \& Turner, R. (1992). Dynamic magnetic resonance imaging of human brain activity during primary sensory stimulation. Proc. Natl. Acad. Sci. U.S.A., 89, 5675-5679.

Lindquist, M.A. \& Gelman, A. (2009). Correlations and multiple comparisons in functional imaging. Persp. Psych. Sci., 4, 310-313. 
Lorentz, G.G. (1976). The 13th problem of Hilbert. In Mathematical developments arising from Hilbert problems (Amer. Math. Soc.), 419-430, Providence, USA.

Lyttelton, O.C., Karama, S., Ad-Dab'bagh, Y., Zatorre, R.J., Carbonell, F., Worsley, K. \& Evans, A.C. (2009). Positional and surface area asymmetry of the human cerebral cortex. NeuroImage, 46, 895-903.

Macey, P.M., Macey, K.E., Kumar, R. \& Harper, R.M. (2004). A method for removal of global effects from fMRI time series. NeuroImage, 22, 360-366.

Madsen, P.L., Hasselbalch, S.G., Hagemann, L.P., Olsen, K.S., Bülow, J., Holm, S., Wildschiødtz, G., Paulson, O.B. \& Lassen, N.A. (1995). Persistent resetting of the cerebral oxygen/glucose uptake ratio by brain activation: evidence obtained with the KetySchmidt technique. J. Cereb. Blood Flow Metab., 15, 485-491.

Malonek, D. \& Grinvald, A. (1996). Interactions between electrical activity and cortical microcirculation revealed by imaging spectroscopy: implications for functional brain imaging. Science, 272, 551-554.

Mandell, D.M., Han, J.S., Poublanc, J., Crawley, A.P., Stainsby, J.A. \& an D. J. Mikulis, J.A.F. (2008). Mapping cerebrovascular reactivity using blood oxygen level-dependent MRI in patients with arterial steno-occlusive disease. Stroke, 39, 2021-2028.

Mazziotta, J., Toga, A., Evans, A., Fox, P., Lancaster, J., Zilles, K., Woods, R., Paus, T., Simpson, G., Pike, B., Holmes, C., Collins, L., Thompson, P., MacDonald, D., Iacoboni, M., Schormann, T., Amunts, K., Palomero-Gallagher, N., Geyer, S., Parsons, L., Narr, K., Kabani, N., Le Goualher, G., Boomsma, D., Cannon, T., Kawashima, R. \& Mazoyer, B. (2001). A probabilistic atlas and reference system for the human brain: International Consortium for Brain Mapping (ICBM). Philos. Trans. R. Soc. Lond. B Biol. Sci., 356, 1293-1322. 
McIntosh, A.R. \& Lobaugh, N.J. (2004). Partial least squares analysis of neuroimaging data: applications and advances. NeuroImage, 23, S250-S263.

McIntosh, A.R., Bookstein, R.L., Haxby, J.V. \& Grady, C.L. (1996). Spatial pattern analysis of functional brain images using partial least squares. NeuroImage, 3, 143-157.

Mourão-Miranda, J., Bokde, A.L.W., Born, C., Hampel, H. \& Stetter, M. (2005). Classifying brain states and determining the discriminating activation patterns: support vector machine on functional MRI data. NeuroImage, 28, 980-995.

Murphy, B.D., Fox, A.J., Lee, D.H., Sahlas, D.J., Black, S.E., Hogan, M.J., Coutts, S.B., Demchuk, A.M., Goyal, M., Aviv, R.I., Symons, S., Gulka, I.B., Beletsky, V., Pelz, D., Hachinski, V., Chan, R. \& Lee, T.Y. (2006). Identification of penumbra and infarct in acute ischemic stroke using computed tomography perfusion-derived blood flow and blood volume measurements. Stroke, 37, 1771-1777.

Murphy, K., Birn, R.M., Handwerker, D.A., Jones, T.B. \& Bandettini, P.A. (2009). The impact of global signal regression on resting state correlations: are anti-correlated networks introduced? NeuroImage, 44, 893-905.

Murphy, T.H. \& Corbett, D. (2009). Plasticity during stroke recovery: from synapse to behaviour. Nat. Rev. Neurosci., 10, 861-872.

Nachev, P., Kennard, C. \& Husain, M. (2008). Functional role of the supplementary and pre-supplementary motor areas. Nat. Rev. Neurosci., 9, 856-869.

Ogawa, S., Lee, T.M., Kay, A.R. \& Tank, D.W. (1990). Brain magnetic resonance imaging with contrast dependent on blood oxygenation. Proc. Natl. Acad. Sci., 87, 9868-9872.

Oppenheim, A.V. \& Schafer, R.W. (1999). Discrete-Time Signal Processing. Prentice Hall, New Jersey, USA, 2nd edn. 
Papoulis, A. (1984). Probability, Random Variables, and Stochastic Processes. McGrawHill, New York, USA.

Penfield, W. \& Rasmussen, T. (1950). The Cerebral Cortex of Man: A Clinical Study of Localization of Function. Macmillan, New York, USA.

Penny, W., Flandin, G. \& Trujillo-Barreto, N. (2007). Bayesian comparison of spatially regularised general linear models. Hum. Brain Mapp., 28, 275-293.

Pineiro, R., Pendlebury, S., Johansen-Berg, H. \& Matthews, P.M. (2002). Altered hemodynamic responses in patients after subcortical stroke measured by functional MRI. Stroke, 33, 103-109.

Politis, D.N. (2003). The impact of bootstrap methods on time series analysis. Stat. Science, 18, 219-230.

Purdon, P.L. \& Weisskoff, R.M. (1998). Effect of temporal autocorrelation due to physiological noise and stimulus paradigm on voxel-level false-positive rates in fMRI. Hum. Brain Mapp., 6, 239-249.

Quiroga, R.Q., Reddy, L., Kreiman, G., Koch, C. \& Fried, I. (2005). Invariant visual representation by single neurons in the human brain. Nature, 435, 1102-1107.

Raiffa, H. \& Schlaifer, R. (1961). Applied Statistical Decision Theory. Harvard Business School, Boston, USA.

Rajapakse, J.C. \& Zhou, J. (2007). Learning effective brain connectivity with dynamic Bayesian networks. NeuroImage, 37, 749-760.

Rajapakse, J.C., Kruggel, F., Maisog, J.M. \& von Cramon, D.Y. (1998). Modeling hemodynamic response for analysis of functional MRI time-series. Hum. Brain Mapp., 6, $283-300$. 
Ramoni, M.F., Sebastiani, P. \& Kohane, I.S. (2002). Cluster analysis of gene expression dynamics. Proc. Natl. Acad. Sci., 99, 9121-9126.

Reis, J., Swayne, O.B., Vandermeeren, Y., Camus, M., Dimyan, M.A., Harris-Love, M., Perez, M.A., Ragert, P., Rothwell, J.C. \& Cohen, L.G. (2008). Contribution of transcranial magnetic stimulation to the understanding of cortical mechanisms involved in motor control. J. Physiol., 586, 325-351.

Roc, A.C., Wang, J., Ances, B.M., Liebeskind, D.S., Kasner, S.E. \& Detre, J.A. (2006). Altered hemodynamics and regional cerebral blood flow in patients with hemodynamically significant stenoses. Stroke, $\mathbf{3 7}, 382-387$.

Rosen, B.R., Buckner, R.L. \& Dale, A.M. (1998). Event-related functional MRI: past, present, and future. Proc. Natl. Acad. Sci., 95, 773-780.

Rossini, P.M., Altamura, C., Ferretti, A., Vernieri, F., Zappasodi, F., Caulo, M., Pizziella, V., Gratta, C.D., Romani, G.L. \& Tecchio, F. (2003). Does cerebrovascular disease affect the coupling between neuronal actvity and local haemodynamics? Brain, 127, $99-110$.

Röther, J., Knab, R., Hamzei, F., Fiehler, J., Reichenbach, J.R., Büchel, C. \& Weiller, C. (2002). Negative dip in BOLD fMRI is caused by blood flow-oxygen consumption uncoupling in humans. NeuroImage, 15, 98-102.

Savadjiev, P., Campbell, J.S., Pike, G.B. \& Siddiqi, K. (2006). 3D curve inference for diffusion MRI regularization and fibre tractography. Med. Image. Analysis, 10, 799813.

Seghier, M.L., Lazeyras, F., Zimine, S., Maier, S.E., Hanquinet, S., Delavelle, J., Volpe, J.J. \& Huppi, P.S. (2004). Combination of event-related fMRI and diffusion tensor imaging in an infant with perinatal stroke. NeuroImage, 21, 463-472. 
Talairach, J. \& Tournoux, P. (1988). Co-Planar Stereotaxic Atlas of the Human Brain: 3-Dimensional Proportional System - An Approach to Cerebral Imaging. Thieme, New York, USA.

Thomas, B.P., Welch, E.B., Niederhauser, B.D., Jr., W.O.W., Anderson, A.W., Gore, J.C., Avison, M.J. \& Creasy, J.L. (2008). High-resolution 7T MRI of the human hippocampus in vivo. J. Magn. Reson. Imaging, 28, 1266-1272.

Tuch, D.S., Reese, T.G., Wiegell, M.R. \& Wedeen, V.J. (2003). Diffusion MRI of complex neural architecture. Neuron, 40, 885-895.

Turner, R.S., Desmurget, M., Grethe, J., Crutcher, M.D. \& Grafton, S.T. (2003). Motor subcircuits mediating the control of movement extent and speed. J. Neurophysiol., 90, 3958-3966.

Vapnik, V., ed. (1998). Statistical Learning Theory. Wiley, New York, USA.

Vazquez, A.L. \& Noll, D.C. (1998). Nonlinear aspects of the BOLD response in functional MRI. NeuroImage, $\mathbf{7}, 108-118$.

Villringer, A. (2000). Physiological changes during brain activation. In C. Moonen \& P. Bandettini, eds., Functional MRI, 3-13, Springer-Verlag, Berlin, Germany.

von Economo, C. \& Koskinas, G.N. (2008). Atlas of the Cytoarchitectonics of the Adult Human Cerebral Cortex. Karger, Basel, Switzerland, translation from German by L. C. Triarhou.

Wang, Z., Childress, A.R., Wang, J. \& Detre, J.A. (2007). Support vector machine learning-based fMRI data group analysis. NeuroImage, 36, 1139-1151.

Ward, N.S., Brown, M.M., Thompson, A.J. \& Frackowiak, R.S.J. (2003a). Neural correlates of motor recovery after stroke: a longitudinal fMRI study. Brain, 126, 2476-2496. 
Ward, N.S., Brown, M.M., Thompson, A.J. \& Frackowiak, R.S.J. (2003b). Neural correlates of outcome after stroke: a cross-sectional fMRI study. Brain, 126, 1430-1448.

Worsley, K.J. \& Friston, K.J. (1995). Analysis of fMRI time-series revisited-again. NeuroImage, 2, 173-181.

Zadeh, L. (1965). Fuzzy sets. Inform. and Control, 8, 338-353.

Zarahn, E., Aguirre, G.K. \& D'Esposito, M. (1997). Empirical analyses of BOLD fMRI statistics. NeuroImage, 5, 179-197.

Zhou, J. \& Rajapakse, J.C. (2008). Fuzzy approach to incorporate hemodynamic variability and contextual information for detection of brain activation. Neurocomputing, $\mathbf{7 1}$, 3184-3192. 
Appendix A

Ethics and Experimental Protocol

A.1 Research ethics board letter of approval 


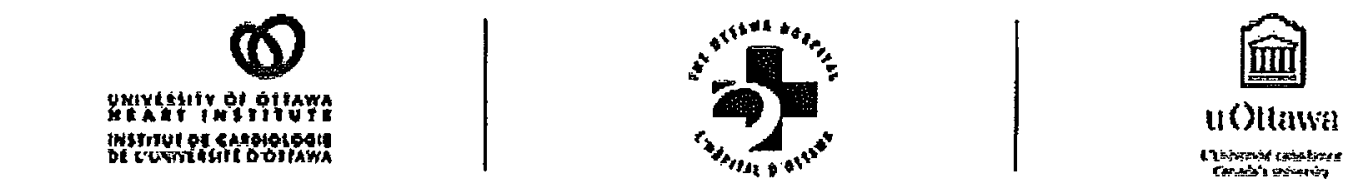

Ottawa Hospital Research Ethics Boards / Conseils d'éthique en recherches

761 Parkdalo Avenue sulte 108, Ottawa, Ontarlo K1Y 157 613-798-6565 oxt. 14902 Fax: 613-761.4311

htto:thww.ohfl.ca/oheob

Thursday, August 13, 2009

Dr. Matthew Hogan

University of Ottawa

451 Smyth Road Room 1442

Roger Guindon Hall

Ottawa, Ontario

K1H 8M5

Dear Dr. Hogan:
Re: Protocol \# 2009395-01H Optimization of an Event-Related Functional Magnetic Resonance Imaging (fMRI) Paradigm Using a Visual Feedback Controlled Motor Task

Protocol approval valid until - Sunday, September 13, 2009

Thank you for the French poster received on August 12,2009. This protocol was reviewed by the full Board of the Ottawa Hospital Research Ethics Board (OHREB) at the meeting held on June 2, 2009. You have met the requirements of the OHREB and your protocol has been granted approval by the OHREB for two months to begin recruiting English-speaking participants. No changes, amendments or addenda may be made to the protocol or the consent form without the OHREB's review and approval.

Approval is for the following:

- Detailed Description of Research Proposal (version 3) received July 15, 2009

- Case Report Form received July 15, 2009

- Hospital MRI Screening Form

- English Poster received July 15, 2009

- Subject Master Record Form received July 15, 2009

- English Participant Questionnaire Form received July 15, 2009

- English Participant Information Letter and Consent Form received July 15, 2009

- French Poster received August 12, 2009

The validation date should be indicated on the bottom of all consent forms and information sheets (see copy attached).

Upon receipt, review and approval of the French consent form and questionnaire, the study expiry date may be extended to June 1, 2009 (one year from the meeting date) and the recruitment of French-speaking participants may begin. When submitting French documentation to the OHREB, confirm it has been translated or approved by Eric Lepine (email all documents, except validated questionnaires, to Eric at elepine@ohri.ca). 
The Ottawa Hospital Research Ethics Board is constituted in accordance with, and operates in compliance with the requirements of the Tri-Council Policy Statement: Ethical Conduct for Research Involving Humans; Health Canada Good Clinical Practice: Consolidated Guideline; Part C Division 5 of the Food and Drug Regulations of Health Canada; and the provisions of the Ontario Health Information Protection Act 2004 and its applicable Regulations.

Yours sificeraly,

Raphael Šaginur, M.L.

Chairman

Ottawa Hospital Research Ethics Board

Encl.

II 


\section{A.2 Protocol involving human subject particiaption}




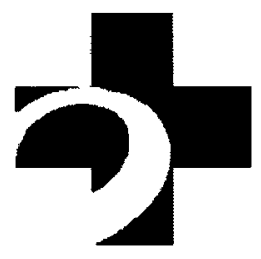

The Ottawa L'Hôpital Hospital $\mid$ d'Ottawa

Research Ethics Board

\section{Application for Research Ethics Approval}

To submit a research proposal to the Ottawa Hospital Research Ethics Board (OHREB), it is mandatory to submit the actual protocol.

The application forms must be filled out completely. Please bear in mind that some members of the OHREB may not be experts in your own field so please strive, where appropriate, to make your submission understandable to those outside your area of expertise. Information Sheets and Consent Form must be included with the protocol (where appropriate).

\section{Please submit the appropriate number of copies:}

For protocols requiring full review:

- Application

- Patient Information Sheet and/or

Consent forms *

18 copies (signed original plus 17 copies)

( ${ }^{*}$ English version only)

18 copies (original plus 17 copies)

(Once the English version has been approved by the OHREB, the French version may be submitted.)

- Departmental Impact Sheet

with applicable attachments

- Detailed Budget:

- Protocol:

18 copies (signed original plus 17 copies)

18 copies

- Questionnaires,

Case Report Forms, etc : $\quad 8$ copies

- Investigator's Brochure: 2 copies

For protocols requiring expedited review:

(Survey, Chart Reviews, Non-Invasive Testing/Intervention):

- Application:

2 copies (signed original plus 1 copy)

- Patient Information Sheet and/or

Consent forms *

2 copies (original plus 1 copy)

- Questionnaires, etc.

( ${ }^{*}$ English version only)

(Once the English version has been approved by the OHREB, the French version may be submitted.)

- Departmental Impact Sheet with

applicable attachments

- Detailed Budget

2 copies (signed original plus 1 copy)

- Protocol

2 copies (original plus 1 copy)

2 copies (original plus 1 copy)

N.B. All documentation must be typed. Incomplete protocols will be returned prior to review. 
The forms have been created in Word, using the Forms tool to allow ease of use in completing them. The following are a few tips on completing the document:

- Please use the "TAB" key to move between fields.

- The "Enter" key will create extra space in the form that is not required.

- The form fields will not accept formatting, normal type will be fine.

- It is hoped that you are able to complete the form in the space allocated, however, if you need additional space, please insert a page where appropriate.

- If you have suggestions to improve this form, please let us know.

\section{Protocol Submission}

The application form, the protocol, investigator's brochure, the consent form, patient information sheet, budget, questionnaires and any other material described above should be submitted to:

Ottawa Hospital Research Ethics Board

c/o Ms. Christine Banyard

Ottawa Hospital, Civic Campus

751 Parkdale Avenue

Suite 106

Ottawa, Ontario K1Y 1J7

or

Ottawa Hospital Research Ethics Board

Drop Box Location: Room 1812-A (in the CEP area on the main floor)

General Campus - Ottawa Hospital

501 Smyth Road, Box 201

Ottawa, Ontario

K1H 8 L6

To confirm whether or not we have received your correspondence, or to request a copy of lost correspondence, please contact:

Kathy Millar

Ethics Clerk, Research Ethics Board

Phone: 613-798-5555, extension 17523

Email:kmillar@ohri.ca

For information on the status of your protocol up to and including initial approval, please contact:

Christine Banyard

Protocol Officer II, Research Ethics Board

Phone : 613-798-5555, extension 14902

Email: cbanyard@ohri.ca

Or 
Linda Longpré

Protocol Officer II, Research Ethics Board

Phone: 613-798-5555, extension 13523

Email: longpre@ohri.ca

For general inquiries about research ethics including the status of revised information sheets, amendments, renewals, terminations, etc., please call:

Heather McDonald

Protocol Officer I, Research Ethics Board

Phone: 613-798-5555, extension 14146

Email: heamcdonald@ohri.ca

For information on procedural issues, problematic protocols, compensation clauses, OHREB document changes, etc. please contact:

Mary Ann Laviolette

Ethics Co-ordinator, Research Ethics Board

Phone: 613-798-5555, extension 15072

Email: mlaviolette@ohri.ca

For information on and requests for clinical research space, please contact:

Kim Adams

Director, Research Administration, OHRI

Phone: 613-798-5555, extension 15079

Email: kadams@ohri.ca

The Chair of the OHREB is:

Raphael Saginur, M.D.

Phone: 613-798-5555, extension 14902

The Vice-Chair of the OHREB is:

Francine F-A. Sarazin, Ph.D., C.Psych.

Phone: 613-798-5555, extension 14902

The Chair of the HREB is:

Richard F. Davies, MD, PhD, FRCPC

Phone: 613-798-5555, extension 19865

The Vice-Chair of the HREB is:

James A. Robblee, MD, FRCPC

Phone: 613-798-5555, extension 19865

Protocols must be signed by the Head of the Department or Division in which the research projects are to be carried out. (The Principal Investigator or Co-Investigator should not sign as the Head of the Department or Division for their own research studies) 
Protocols will be reviewed on a bi-monthly basis at the OHREB meeting. Please refer to the list for the exact submission deadlines listed under 'Meeting Dates'.

Protocols submitted for 'Expedited Review' are normally processed within two to four weeks of submission. Applications may be submitted at any time - the submission dates do not apply to 'expedited' studies.

Protocols submitted for 'Chart Review' are normally processed within 24 to 48 hours of submission. Applications may be submitted at any time - the submission dates do not apply to 'chart review' studies. 


\section{RESEARCH ETHICS APPLICATION \\ NOTE: ALL DOCUMENTATION MUST BE TYPE WRITTEN. \\ Use the "TAB" key to move between fields.}

\section{PROTOCOL TITLE}

Optimization of an event-related functional magnetic resonance imaging (fMRI) paradigm using a visual feedback controlled motor task

\section{PRIMARY INVESTIGATOR FOR MULTICENTRE TRIALS}

\begin{tabular}{|l|l|l|}
\hline Last Name & First Name \\
\hline Title/Position & $\begin{array}{l}\text { Department } \\
\text { \& Location } \\
\text { (or full } \\
\text { mailing } \\
\text { address) }\end{array}$ \\
\hline Tel. & Fax & \\
\hline Email & & \\
\hline
\end{tabular}

\section{PRINCIPAL INVESTIGATOR AT THE OTTAWA HOSPITAL}

If the Principal Investigator does not hold an academic appointment, indicate who the responsible investigator will be. Investigators must complete the Tri-Council Policy Statement On-lineTutorial and submit a copy of their certificate to Hillary Falconer at hfalconer@ohri.ca. The tutorial can be found at: www.pre.ethics.gc.ca

\begin{tabular}{|c|c|c|c|}
\hline Last Name & Hogan & First Name & Matthew \\
\hline \multirow[t]{2}{*}{ Title/Position } & \multirow[t]{2}{*}{ Associate Professor } & Tel. & (613)798-5555 ext.16251 \\
\hline & & Fax & $(613) 761-5360$ \\
\hline $\begin{array}{l}\text { Dept/Unit \& } \\
\text { Location } \\
\text { (full mailing } \\
\text { address) }\end{array}$ & $\begin{array}{l}\text { Division of Neurosciences, C2 } \\
\text { The Ottawa Hospital - Civic } \\
\text { Campus } \\
1053 \text { Carling Avenue } \\
\text { Ottawa, Ontario K1Y 4E9 }\end{array}$ & Email & mhogan@uottawa.ca \\
\hline $\begin{array}{l}\text { Division/ } \\
\text { Portfolio }\end{array}$ & Neurology & Signature: & \\
\hline
\end{tabular}

\section{CO-INVESTIGATORS AT THE OTTAWA HOSPITAL}

\begin{tabular}{|c|c|c|c|}
\hline Last Name & Nguyen & First Name & Thanh \\
\hline \multirow[t]{2}{*}{ Title/Position } & \multirow[t]{2}{*}{ Assistant Professor } & Tel. & (613)594-2490 ext. \\
\hline & & Fax & $(613) 761-4529$ \\
\hline $\begin{array}{l}\text { Dept/Unit \& } \\
\text { Location } \\
\text { (full mailing } \\
\text { address) }\end{array}$ & $\begin{array}{l}\text { Diagnostic Imaging } \\
\text { The Ottawa Hospital } \\
\text { Civic Campus } \\
1053 \text { Carling Ave } \\
\text { Ottawa, ON, K1Y 4E9 }\end{array}$ & Email & thnguyen@ottawahospital.on.ca \\
\hline $\begin{array}{l}\text { Division/ } \\
\text { Portfolio }\end{array}$ & Neuroradiology & Signature: & \\
\hline
\end{tabular}




\begin{tabular}{|c|c|c|c|}
\hline Last Name & Cameron & First Name & lan \\
\hline \multirow[t]{2}{*}{ Title/Position } & \multirow[t]{2}{*}{ MR Physicist } & Tel. & (613)737-8635 ext. \\
\hline & & Fax & $(1)-$ \\
\hline $\begin{array}{l}\text { Dept/Unit \& } \\
\text { Location } \\
\text { (full mailing } \\
\text { address) }\end{array}$ & $\begin{array}{l}\text { Diagnostic Imaging } \\
\text { The Ottawa Hospital } \\
\text { General Campus } \\
501 \text { Smyth Road } \\
\text { Ottawa, ON, K1H 8L6 }\end{array}$ & Email & icameron@ottawahospital.on.ca \\
\hline $\begin{array}{l}\text { Division/ } \\
\text { Portfolio }\end{array}$ & MRI & Signature: & \\
\hline
\end{tabular}

\begin{tabular}{|c|c|c|c|}
\hline Last Name & Sharma & First Name & Mukul \\
\hline \multirow{2}{*}{ Title/Position } & \multirow{2}{*}{$\begin{array}{l}\text { Director, Regional Stroke } \\
\text { Program }\end{array}$} & Tel. & (613)798-5555 ext. 14709 \\
\hline & & Fax & $(613) 761-5360$ \\
\hline $\begin{array}{l}\text { Dept/Unit \& } \\
\text { Location } \\
\text { (full mailing } \\
\text { address) }\end{array}$ & $\begin{array}{l}\text { Division of Neurosciences, C2 } \\
\text { The Ottawa Hospital - Civic } \\
\text { Campus } \\
1053 \text { Carling Avenue } \\
\text { Ottawa, Ontario K1Y 4E9 }\end{array}$ & Email & msharma@ottawahospital.on.ca \\
\hline $\begin{array}{l}\text { Division/ } \\
\text { Portfolio }\end{array}$ & Neruology & Signature: & \\
\hline
\end{tabular}

\begin{tabular}{|l|l|l|l|}
\hline Last Name & First Name & \\
\hline Title/Position & Tel. & $(\quad)$ & - \\
\hline $\begin{array}{l}\text { Dept/Unit \& } \\
\text { Location } \\
\text { (full mailing } \\
\text { address) } \\
\text { Division/ } \\
\text { Portfolio }\end{array}$ & Fax & Email & \\
\hline
\end{tabular}

\begin{tabular}{|c|c|c|c|c|}
\hline Last Name & First Name & & & \\
\hline \multirow[t]{2}{*}{ Title/Position } & Tel. & $(\quad)$ & - & ext. \\
\hline & Fax & $(\quad)$ & - & \\
\hline $\begin{array}{l}\text { Dept/Unit \& } \\
\text { Location } \\
\text { (full mailing } \\
\text { address) }\end{array}$ & Email & & & \\
\hline $\begin{array}{l}\text { Division/ } \\
\text { Portfolio }\end{array}$ & Signature: & & & \\
\hline
\end{tabular}

If you need more space an additional form for co-investigators is available on the website. 


\section{APPROVAL BY INVESTIGATOR'S DEPARTMENT/DIVISION HEAD/ \\ CLINICAL MANAGER/CHIEF (THIS SHOULD NOT BE THE PRINCIPAL INVESTIGATOR AND/OR CO-INVESTIGATOR)}

Hospital and university division and department administrators are responsible for academic activities within their unit, and may provide guidance and support to investigators. The purpose of this signature section is to ensure that administrators are aware of research activities and the impact of these activities.

$\square \quad$ The study answers a reasonable scientific/clinical question and is consistent with hospital/faculty policies and mission.

$\square$ The study resources (budget, space, support staff) are adequate to support the study.

$\square \quad$ The local investigators are qualified to perform the study.

$\square \quad$ There are an adequate number of research participants suitable to be approached for enrolment for this study. This population is not already oversubscribed in clinical research.

I have reviewed this application and agree it should be submitted for ethics approval.

\begin{tabular}{|l|l|l|} 
Name & Pierre Bourque & Contact Number : (613)798-5555 ext. 18160 \\
\hline Title/Position & Head, Division of Neurology & Signature \\
$\begin{array}{l}\text { Dept/Unit \& } \\
\text { Location }\end{array}$ & $\begin{array}{l}\text { Division of Neurosciences, C2 } \\
\text { The Ottawa Hospital - Civic }\end{array}$ & \\
(full mailing & $\begin{array}{l}\text { Campus } \\
\text { address) }\end{array}$ & $\begin{array}{l}\text { 1053 Carling Avenue } \\
\text { Ottawa, Ontario K1Y 4E9 }\end{array}$ \\
\hline Date &
\end{tabular}

\section{REVIEW TYPE}

Please indicate whether you are requesting full or expedited review. (Please see our website www.ohri.ca/ohreb for more information on what qualifies for expedited review.)

\section{Full Review \\ X Expedited Review}

\section{STUDY TYPE}

Describe the Research Project by checking as many of the following as apply:

Study Type:

\begin{tabular}{|l|l|}
\hline$\square$ & Investigator driven and sponsored by OHRI \\
\hline $\mathbf{X}$ & Experimental Research/Clinical Trial \\
\hline & Interventional Research \\
\hline $\mathbf{X}$ & Non-Interventional Research \\
\hline $\mathbf{X}$ & Observational Research \\
\hline $\mathbf{X}$ & Pilot \\
\hline & Sequel to previously approved project (Protocol \# : _ _ \\
\hline & Genetic Research (Addendum 1 must be included with completed application) \\
\hline
\end{tabular}


Program Evaluation

New therapeutic method

Medical Device Research

Please attach a letter from sponsor indicating Health Canada application/approval.

This is mandatory prior to final REB approval.

Health Canada Application/Approval is attached (insert as next page)

Health Canada Application/Approval will be forwarded.

\section{Location of the Study:}

$X \quad$ Single Centre Trial

Multicentre Trial

Study Design:

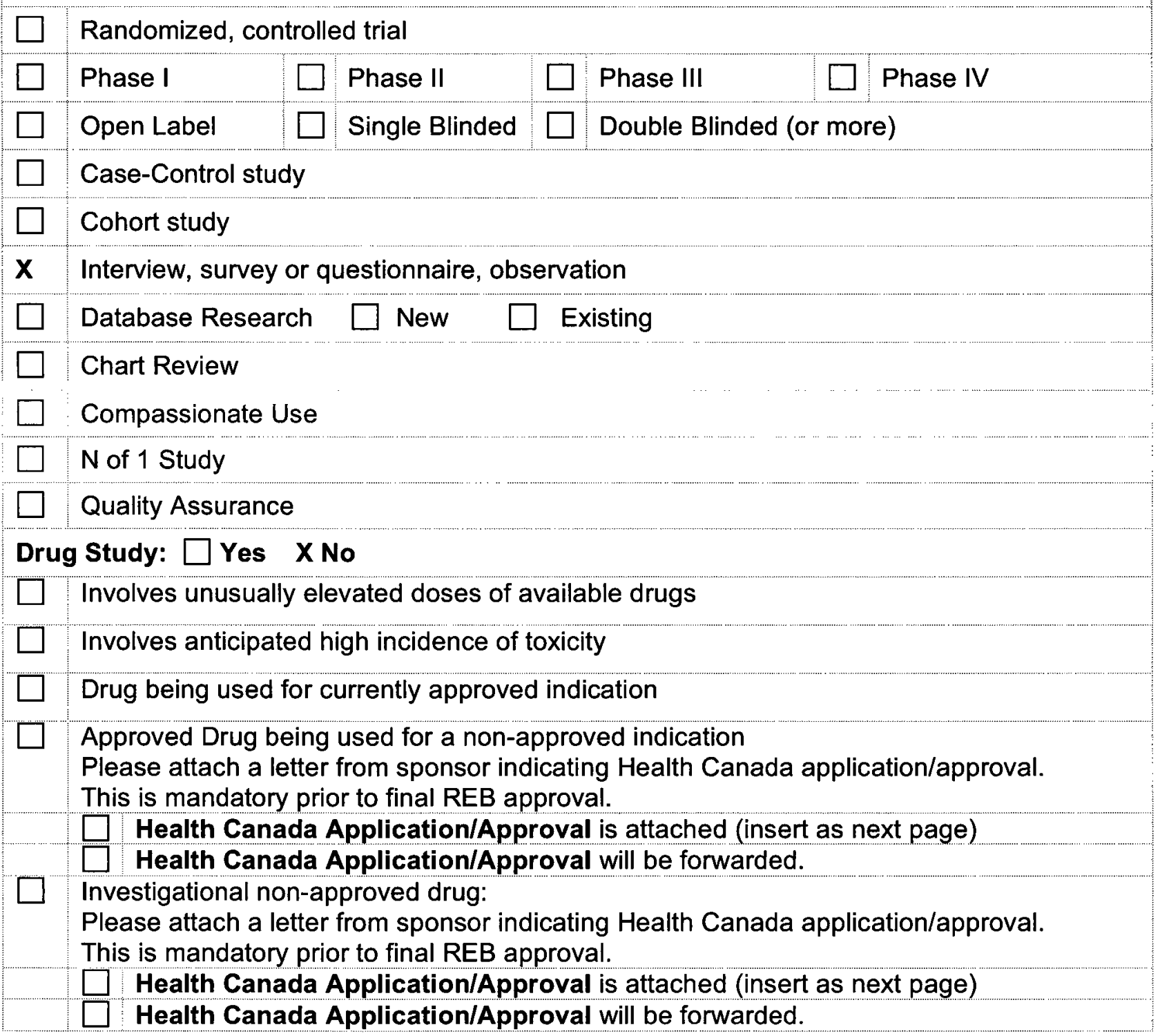




\section{PURPOSE AND OBJECTIVES}

Please state clearly the hypothesis to be tested, in lay terms.

The purpose of this observational study is two-fold:

1. to determine the optimal parameters for our stimulation protocol for fMRI studies, and

2. to assess the reproducibility of the acquired fMRI data in 10 healthy subjects.

The stimulus protocol requires the subject to respond to brief, computer generated visual cues displayed on a screen by squeezing a small plastic ball placed in their hand. Much like a video game, the ball is wired back to the computer so that the display provides visual feedback to the subject on their actions.

The objectives for part (1) are to determine the following parameters:

a. a suitable placement of the screen inside the scanner room and suitable ambient illumination and display colours,

b. a suitable fraction of the subject's maximum applied force in order to produce a clear signal yet not cause discomfort or unnecessary motion, and

c. a suitable stimulus duration to produce a clear signal yet remain as brief as possible.

The objectives for part (2) are to validate the protocol by assessing the reproducibility of the fMRI data acquired from 10 healthy subjects. This will be done by comparing two sessions taken 3 months apart for each subject. 
Provide the rationale for the study.

It is planned to use the optimized stimulus protocol to collect fMRI data evaluating brain function in future clinical studies. The reproducibility assessment in healthy subjects will be used to estimate the required sample size of patient populations and appropriate data analysis methods.

A detailed description of the research proposal is found in appendix $E$.

\section{RECRUITMENT}

Describe how these research participants will be identified and recruited. (Only members of the participant's health care team should contact patients at the Ottawa Hospital.)

In addition, please ensure you address the following:

- If initial contact is by letter or if an advertisement is to be used, attach a copy.

- How will the researcher ensure that there are no breaches of patient privacy?

- How will the possibility of coercion, duress or undue incentive be avoided or minimized?

.

The subjects will be recruited from a population of convenience, consisting of individuals working in our imaging and stroke research groups, by use of advertisement (appendix A) posted in these research areas at the OHRI/TOH. No patients will be recruited and no advertisement will be posted in areas where they would be seen by patients.

All data will be anonymized using an alphanumeric code. A copy of the key table and subject master records (Apppendix $G$ ) linking the subject name to this code will be held by the principal investigator and kept in a locked file cabinet. Results will be reported in aggregate with no reference to individual subject names.

Coercion will be minimized by using only posted advertisement in the recruitment process. Potential subjects will be informed that they are participating on a strictly voluntary basis and no incentive will be provided. 
Are recruitment incentives provided? $\square$ Yes $X$ No

If yes, please describe what they are, and when they will be provided.

Are controls involved? $\square$ Yes X No

Does the study include subjects in a control group? $\square$ Yes $\square$ No

If controls are involved, if their selection and/or recruitment differs from the above, provide details.

Does the research include research participants who may not be competent to give informed consent? $\square$ Yes $X$ No If yes, justify and explain how consent will be obtained and from whom.

- Number of Centres recruiting globally: 1

X National Study - Country Canada

International Study - Country

- Total number of research participants being recruited at all centres globally: 16 
- Number of research participants to be recruited at each Ottawa Hospital campus:

$\square$ Civic $\mathrm{X}$ General

Riverside

Other 16

Provide the rationale for the selected sample size and the methodology used to calculate the sample size:

This is an observational study to evaluate the reproducibility of $f M R I$ data acquired with our stimulus protocol. The variability in the data is not known. The sample sizes proposed are based on our prior experience with $\mathrm{fMRI}$ studies performed at the Ottawa Hospital and are reasonable for the purposes of this study.

Part 1: Optimization of parameters (see Appendix E for details)

a. The investigators will determine the positioning of the screen and illumination settings.

b. The optimal force to apply to the pressure balls to comfortably generate a clear fMRI signal will be determined by 3 subjects.

c. The optimal stimulus duration will be determined using data from 6 subjects ( 3 subjects in part $b$ above plus 3 new subjects).

Part 2: Assessment of signal reproducibility (see Appendix $E$ for details)

We do not yet have good estimates on the variability of brain response measured with fMRI using our novel stimulation paradigm. Based on our experience using fMRI in block design paradigms, data from 10 subjects should be sufficient to evaluate signal reproducibility. 


\section{DESCRIPTION OF POPULATION}

Inclusion Criteria - Who is being recruited and what are the criteria for their selection?

We are recruiting normal individuals who see the posted advertisement and respond. This will include researchers, students, research support staff, technologists and technicians.

The inclusion criteria are:

1) Age 20 to 65

2) Informed consent obtained 
Exclusion Criteria - What research participants are excluded from participation?

1) MR imaging exclusion criteria as per The Ottawa Hospital MRI Screening Form (appendix B)

2) Pregnancy

3) Inability to perform the required task

4) Diabetes, uncontrolled hypertension, COPD, CHF, other significant concurrent medical or surgical disease

5) Prior symptomatic neurological disease resulting in deficit, cognitive changes, or impairment of function

6) Seizure in past 12 months

7) Migraine in past week

8) History of alcohol or drug abuse

9) Inability to tolerate MR imaging (claustrophobia, anxiety) 


\section{DESCRIPTION OF METHODS AND PROCEDURES}

If additional space is required, insert one additional page)

Summary of Methods and Procedures. Please include a summary of the following:

- any specific manipulations;

- type, quantity, and route of administration of drugs and radiation; operations; tests; use of medical devices that are prototype or altered from those in clinical use;

- type, and number of interviews or questionnaires; are the questionnaires validated, reliable and have they been pilot tested?

- Are procedures/treatments standard practice or new, what is the risk level? (Flow diagrams and point form discussion are encouraged and should be appended separately.)

\section{Recruitment interview:}

Potential subjects will be provided with the study information letter (Appendix $C$ ) and, following review, informed consent to participate in the study will be obtained by one of the study investigators. Subject specific information will be collected (Appendix $G$ ) and study participants will then complete a short questionnaire (appendix $D$ ) to determine eligibility to continue in the study. If eligible, subjects will then participate in either Part 1 or Part 2 of this study.

\section{Part 1: Optimization of parameters}

a. The investigators will meet in the scanner room to establish a suitable experimental environment. No scanning will be necessary.

b. Three healthy subjects will each spend 45 minutes (including preparation time) in the MR scanner during which they will undergo 15 minutes of $\mathrm{fMRI}$ scanning, followed by a 5 minute anatomical scan, and then another 15 minutes of fMRI. These same subjects will also participate in part (c), however, no sooner than 3 months later in order to minimize any learning effects.

c. Six healthy subjects will each spend $\mathbf{4 5}$ minutes (including preparation time) in the scanner during which they will undergo the same scanning schedule as in part (b).

\section{Part 2: Assessment of signal reproducibility}

Ten healthy subjects who did not participate in part (1) will each attend 2 MR imaging sessions approximately 3 months apart. During each session, the subject will first complete a 15 minute training routine to measure their maximal applied grip force and their stimulus response timing. Afterwards, they will spend 35 minutes (including preparation time) in the MR scanner during which they will undergo 10 minutes of fMRI scanning, followed by a 5 minute anatomical scan, and another 10 minutes of $f M R I$ scanning. During scanning, the subject's end-tidal $\mathrm{CO} 2$ concentration will be monitored using nasal prongs. Before and after each session, the subject's blood pressure and heart rate will be measured.

All MRI scans will follow standard practice protocols and no contrast agents will be used. Blood pressure will be measured non-invasively using a pressure cuff applied to the arm. End-tidal $\mathrm{CO} 2$ will be measured using nasal prongs connected to a standard capnometer.

The risk level of participation is low; the risks involved apply generally to standard MRI scanning (see section 12 below). The subject is free to withdraw from the study at any time.

A detailed description of the experimental procedure is found in the research proposal (appendix E) and the case report form for each subject is found in appendix $F$. 


\section{RISKS}

Describe the discomfort or risks that participants may incur as a result of their participation in this research. Also note the following:

- particular risks associated with each procedure, drug, test or other aspect of the protocol.

- delineate, when appropriate, what risks relate to standard care,

- what risks relate to participation in the study.

- quantify risks where possible by providing percentages, or by describing as rare, common, etc.

The risks are those associated with standard MRI:

- Infrequent development of temporary heightened anxiety and/or claustrophobia due to loud noises and small enclosure inside the MRI scanner

- Infrequent sensations of discomfort from prolonged period of lying still inside the scanner

- Very rare tapping or tingling sensations in skeletal muscle due to MR pulse sequences; these have not been observed in our studies to date.

- Very rare unrecognized contraindication to MRI; all subjects will undergo standardized screening (appendix B) and will be excluded from study if there is any suspicion of possible contraindication.

- Incidental findings of potential clinical relevance occur in $0.5 \%$ to $2 \%$ of the general population; the subject will be advised of any noted findings and given access to standard clinical evaluation and care if desired.

Will the management of the participant's condition be prolonged or delayed as a result of the research? $\square$ Yes X No

If yes, explain, specifying any risks associated with prolongation or delay.

Are there any standard therapies, diagnostic procedures or information to be withheld from participants for the purpose of the study? $\square$ Yes $X$ No If yes, explain, specifying the risks and benefits to the participants and justify. 
Are there stopping rules for the study? (This does not apply to individual patient withdrawal, but to the study as a whole) $\square$ Yes $X$ No

If yes, please describe.

Is there a data safety monitoring board in place? $\square$ Yes $X$ No

If yes, describe the composition of the board.

What procedures in this protocol are additional to those required for routine patient care? (Include the Impact Sheet with applicable attachments for all procedures which are attributable to this study)

None 
Is a placebo used? $\square$ Yes X No

If yes, please justify:

\section{POSSIBLE BENEFITS}

Describe any possible benefits to the participant as a result of their participation in this research.

There is no direct benefit obtained from participating in this study.

\section{CONFIDENTIALITY}

\section{It is the policy of the OHREB that no records with the patient's name leave the Ottawa Hospital.}

How will data be protected against breaches in security/privacy? Be sure to include the following information:

If the information will be housed in a database, will it be password protected?

$\square$ Confirm that an independent study number will be assigned to each patient record.

What information will be collected? (A 'Case Report Form' may be provided with these details). No identifying information should be used such as hospital unique number, OHIP \#, etc. if any identifying information is required, justification must be provided.

Date of birth is also considered a personal identifier. Confirm that you are not using date of birth. If it is required, clarify whether month and year of birth will suffice? If you require full date of birth, justification must be provided.

$\square$ Indicate how the master list linking the patient to the independent study number will be maintained and safeguarded, and confirm that it will be stored separately and securely.

$\square$ Indicate how data containing Personal Health Information (PHI) will be protected against breaches of privacy. (i.e. locked cabinets? password protected?)

$\square$ Indicate who will have access to the study data, and which organizations and/or individuals will have access to PHI for audit purposes. Add the OHREB and Ottawa Health Research Institute (OHRI) to the list of possible reviewers.

$\square$ Confirm that no PHI will be leaving the Ottawa Hospital. 
Indicate how long information will be kept after the close of the study? (25 years for investigational drug and device studies regulated by Health Canada as outlined in Health Canada's Food and Drug Regulations, Division 5 - Drugs for Clinical Trials Involving Human Subjects, Section C.05.012. For all other studies, records must be kept by the investigator for 15 years.)

Indicate how paper and electronic data will be destroyed once the storage date has expired (shredded? deleted?)

All data will be anonymized using an alphanumeric code that is assigned independently (contains no information that may be linked to the subject's identity). Electronic databases containing study information will be password protected. The information collected on each subject is limited to the subject specific information on the subject master record (Appendix $G$ ) and to that information obtained on the Ottawa Hospital MRI Screening Form (Appendix B), the Screening Questionnaire (Appendix D), and the Case Report Form (Appendix F). The date of birth is not requested. We do require the age at study enrollment. Personal health information is contained in Appendix $B$ and Appendix $D$ which will be held by the principal investigator in a locked filing cabinet. The key table and subject master records (Appendix G) linking the subject name to the study code will be held separately by the principal investigator also in a locked filing cabinet.

The study investigators will have access to the $\mathrm{PHI}$ information collected in Appendices $\mathrm{B}$ and $\mathrm{D}$ which will be identified only by the code string. The CRF data (Appendix F) will be held in a database accessible to the study investigators and password protected. Information on age, sex, handedness, vascular disease and medication use collected from Appendix $D$ will also be stored electronically in this database. Records containing PHI will not leave the Ottawa Hospital.

The OHREB and Ottawa Health Research Institute will have access to the records for audit purposes.

Records will be held by the principal investigator for 15 years. After this time paper records will be destroyed by shredding. Electronic records will be deleted and backup copy media destroyed. 


\section{BUDGET}

\begin{tabular}{|l|l} 
Has this research been funded? & $\square$ YES X NO \\
\hline If YES provide the: & X If NO complete the following: \\
Name of the Agency/Sponsor: & Has funding been applied for: $X$ YES $\square$ NO \\
Amount of funding received: $\$$ & $\begin{array}{l}\text { Name of the Agency/Sponsor: Heart and Stroke Centre } \\
\text { for Stroke Recovery }\end{array}$ \\
Amount of funding applied for: $\$ \underline{30,000}$ \\
Date submitted: $\frac{20 / 04 / 2009}{D D / M M / Y Y Y Y}$
\end{tabular}

If no funding has been applied for, how will the research be supported?

If the study was peer-reviewed, please provide the name of the individual or agency that completed the review.

Does the industry-sponsored budget contain appropriate overhead?

YES $\square$ NO X N/A

A Clinical Trial Agreement has been forwarded to the Contracts Officer

A Clinical Trial Agreement is attached to the OHREB application

Have you included a budget summary for the protocol?

$X$ YES $\square$ NO $\square$ N/A

(If this is not included, approval of protocol will be delayed.

Details on the disbursement of any excess funds received for this study should also be provided.)

Does the REB review fee apply to this study?

YES $\square$ NO X N/A

A cheque is enclosed

An invoice request is enclosed

A transfer request is enclosed

(All industry sponsored research projects are subject to a $\$ 3,000.00$ administration fee, and any cooperative group studies that are funded in excess of $\$ 3,000.00$ per patient.)

\section{POTENTIAL CONFLICTS OF INTEREST}

Please indicate if you have a conflict of interest or separate financial agreements with the sponsor of this study.

$\square$ YES $\square$ NO X N/A If yes, please explain. 


\section{STAFFING}

What staffing will be required? All staff should be listed and this list maintained during the course of the study. Staff must complete the Tri-Council Policy Statement On-lineTutorial and submit a copy of their certificate to Hillary Falconer at hfalconer@ohri.ca. It can be found at www.pre.ethics.gc.ca

This includes research nurses, research co-ordinators, etc. Research nurses should provide a copy of their Nursing Certificate for the current year, to the Research Services Office if this has not already been done for another study.

Name \& Role in Study
Already Employed?
Full-time vs.
Part-time

X FT

NO

PT

$\begin{array}{ll}\text { X YES } & \text { X FT } \\ \square \text { NO } & \square \text { PT }\end{array}$

Laberge

Ph.D. Graduate Student

Data Analyses

2.

3.

4.

5.

$\square$ YES

NO

YES

No

YES

NO

YES

NO

PT

FT

$\square$ PT
Office/Laboratory Location and Telephone
Number

If new office or laboratory space is required, the Space Planning \& Management section of the Departmental Impact form must be signed and submitted Campus: General

\section{Office/Lab \# 1444 RGN}

(613) $562-5800 \times .8182$

\section{Campus:}

\section{Office/Lab \#}

( ) - $\mathbf{x}$.

Campus:

\section{Office/Lab \#}

( ) - $\mathbf{x}$.

Campus:

Office/Lab \#

( ) - $x$

Campus:

\section{Office/Lab \#}

$\mathbf{x}$.

In the space below please provide:

a) a list of their qualifications: BSc, BEng, MASc

b) a list of the duties to be performed, including delegated/sanctioned medical acts and acts performed under the Regulated Health Professions Act if this has not already been done for another study:

None, no clinical duties, no subject contact 
Protocol Title:

\section{DEPARTMENTAL IMPACT}

Does the protocol require use of Hospital and/or OHRI resources (equipment, staff, space) over and above those normally required in the standard care of a patient?

$\begin{array}{lll}\text { Equipment } & \square \text { YES } & \text { X NO } \\ \text { Staff } & \square \text { YES } & \text { X NO } \\ \text { Space } & \square \text { YES } & \text { X NO }\end{array}$

Will hospitalization or outpatient visits be required beyond what is required for standard care?0
Outpatient Visits
$\square$ YES
$X$ NO
Hospitalization
YES
$X$ NO

Indicate impacts associated with this Protocol, by Department:

IF YES is indicated, a signature of an individual authorized to sign for the department must be obtained. (Please see our website for a list of contact names http://www.ohri.ca/ohreb/)

Signature:

\begin{tabular}{|c|c|c|}
\hline Nursing & $\square$ YES & $\times$ NO \\
\hline Emergency Department & $\square$ YES & $X$ NO \\
\hline $\begin{array}{l}\text { Health Records } \\
\text { (See Appendix A) }\end{array}$ & YES & $X$ NO \\
\hline Laboratory Services & $\square$ YES & $X$ NO \\
\hline $\begin{array}{l}\text { Radioisotopes } \\
\qquad \text { (See Appendix B) }\end{array}$ & YES & $X$ NO \\
\hline $\begin{array}{l}\text { Diagnostic Imaging } \\
\text { (See Appendix B and C) }\end{array}$ & YES [ & \\
\hline Pharmacy & $\square$ YES & $X \mathrm{NO}$ \\
\hline Nutrition And Food Services & $\square$ YES & $X \mathrm{NO}$ \\
\hline Opthalmology & $\square$ YES & $X \mathrm{NO}$ \\
\hline Space Planning \& Management & $\square$ YES & $X \mathrm{NO}$ \\
\hline Clinical Investigation Unit & $\square$ YES & $X$ No \\
\hline Cardiopulmonary Services & $\square$ YES & $\mathrm{XNC}$ \\
\hline
\end{tabular}

Please submit Clinical Director's
Acknowledgment
www.ohri.ca/ohreb/forms.htm

\begin{tabular}{l} 
Please submit signed Health Records \\
form. www.ohri.ca/ohreb/forms.htm \\
\hline Please submit signed Lab Impact \\
form. www.ohri.ca/ohreb/forms.htm \\
\hline Please submit Radiation Safety form. \\
www.ohri.ca/ohreb/forms.htm \\
\hline Please submit signed Diagnostic \\
Imaging form. \\
www.ohri.ca/ohreb/forms.htm \\
\hline Please submit signed Pharmacy form \\
www.ohri.ca/ohreb/forms.htm \\
\hline \\
Please submit signed Opthalmology \\
form www.ohri.ca/ohreb/forms.htm \\
\hline
\end{tabular}


APPENDIX A. ETHICS AND EXPERIMENTAL PROTOCOL

\section{A.3 Consent form and supporting documents}




\section{VOLUNTEERS NEEDED FOR MRI BRAIN STUDY*}

The Neuroscience Program of the Ottawa Hospital Research Institute is looking for healthy volunteers between the ages of 20 and 65 to participate in a functional Magnetic Resonance Imaging (MRI) study to determine the quality and reproducibility of brain activity images taken during a visually-cued hand movement task.

Your participation will involve one 10 minute screening interview and either one or two 45 minute MRI sessions scheduled during evenings at the Ottawa Hospital-General Campus. The protocol is non-invasive and only requires performing hand movements while watching a screen during MRI scans.

If you are interested, please contact the Stroke Research Office at the Ottawa Health Research Institute at 613 798-5555 ext 16217.

* This research study has been approved by the Ottawa Hospital Research Ethics Board (OHREB) 


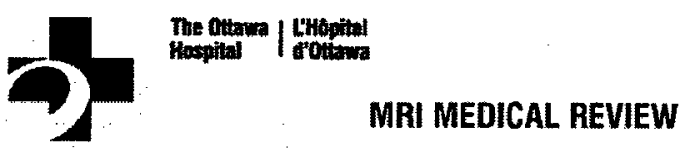

Please answer all questions carefully and completely. The following items can interiere with the MRI study and your safety.

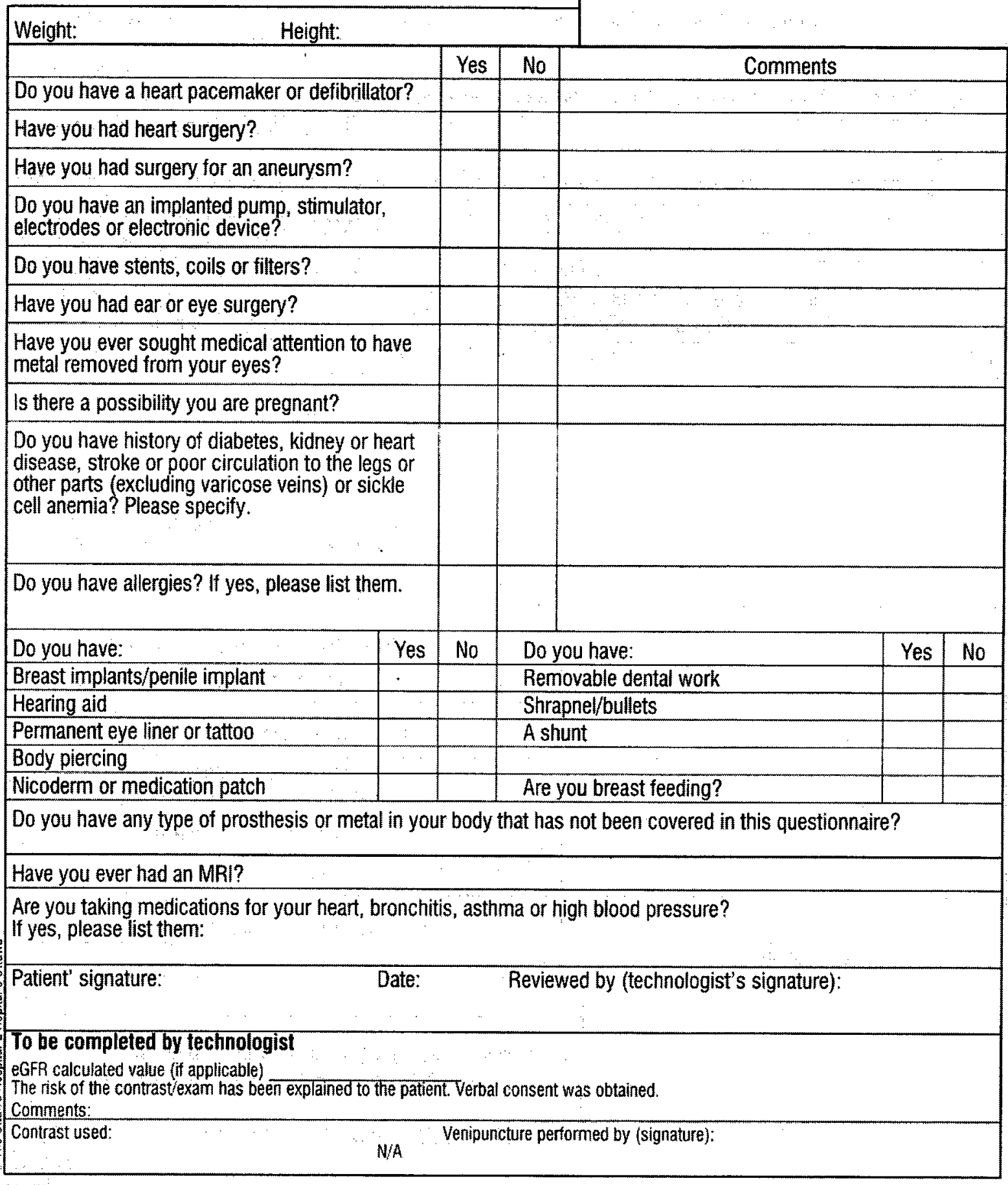




\section{Participant Information Letter}

\section{Optimization of an event-related fMRI paradigm}

using a visual feedback controlled motor task

A pilot study to determine the diagnostic potential of functional MRI for identifying cerebrovascular reactivity and interconnectivity of responding regions

Principal Investigator: Dr. Matthew J. Hogan

This participant information letter is part of the process of obtaining informed consent. This letter should give you the basic idea of what this research study is about and what your participation involves. Please read the information below carefully and ask questions about anything you don't understand before deciding whether or not you wish to take part in this study. You may take home an unsigned copy of this letter to think about and discuss with others before making your decision.

\section{Introduction: brain function and fMRI}

Recent advancements in magnetic resonance imaging (MRI) allow us to image the activity in the brain that takes place during a scan. This is possible because MRI is sensitive to changes in oxygen saturation in blood flowing through the brain. Since oxygen is consumed in active areas, brain activity can be observed non-invasively using MRI. This is called functional MRI (fMRI).

\section{What is the purpose of this study?}

The purpose of this observational study is to assess the diagnostic potential of fMRI to identify regions of the brain that respond during a simple motor task (described on the next page) and determine how each of the regions seen are connected.

\section{How will I participate should I decide to take part in this study?}

In addition to this interview, your participation would consist of either one or two visits, of about one hour duration, to take place in the evening at the Ottawa Hospital-General Campus. If you are scheduled for two visits, they will be approximately 3 months apart.

You will have to follow the instructions listed before your arrival at the hospital:

- do not drink any alcoholic beverages 12 hours before arrival

- do not drink any caffeinated beverages 4 hours before arrival

- do not smoke 4 hours before arrival

- do not perform vigorous exercise 4 hours before arrival

Upon arrival at the hospital, you will be taken to the MRI imaging facility and asked to remove any jewellery. You will be asked if you followed the above instructions.

Before entering the MRI room, you will be briefly trained on how to respond to the visual cues during the fMRI session by squeezing one of the small plastic balls placed in your 


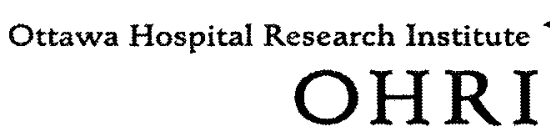

Institut de recherche de l'Hôpital d'Ottawa

hands. You will get to run through a trial-run once outside and once inside the MRI room.

Inside the MRI room, you will be given ear plugs for the loud noises during scanning. You will also be given a plastic tube to wear just under your nose in order to monitor your breathing. You will then be positioned on your back inside the MRI scanner, and the plastic balls placed in your hands. A small mirror will be placed a few centimetres above your eyes so you can see the projection screen placed near your feet. During the fMRI sessions, two horizontal bars will be displayed on a dark blue background. At the beginning, a yellow bar will appear at the bottom of the screen and occasionally will quickly jump to the top of the screen for a couple of seconds. A turquoise bar will also appear at the bottom of the screen. You will be able to move the turquoise bar upwards by squeezing one of the plastic balls. When releasing the ball, the bar will fall back to the bottom of the screen. The objective of the task is to chase the yellow bar with the turquoise bar.

The MRI scanning time is divided into three components:

1. 10-15 minutes of fMRI where you will perform the task

2. 5 minutes of standard MRI where you need only lie still

3. 10-15 minutes of fMRI where you will perform the task

Immediately before and after MRI, your heart rate and blood pressure will be recorded as would be done during a standard visit to your doctor. This will complete your participation in the study. You will be taken back to the change room and thanked as you leave.

\section{Will I be paid to participate?}

No. You will not receive any financial compensation.

\section{What are the potential physical benefits for me?}

There are no direct physical benefits to you.

\section{What are the possible risks of participating?}

The only risks associated with this study are those related to MRI. There are infrequent cases where people feel anxiety or claustrophobia due to the loud noises and small enclosure inside the scanner, or discomfort from lying still for too long. There have been rare cases reported that during MRI, subjects felt the irregular occurrence of tapping or tingling sensations in back or chest muscles or in the joints of the arms or legs. However, this has never been reported during our studies.

No radiological dyes or contrast agents are administered in this study.

What happens if you accidentally discover a problem in my brain?

Unexpected abnormalities may be found in images of the brain in up to $2 \%$ of healthy people. If an abnormality is seen within your images, you will be notified immediately. If you wish, you will receive appropriate medical care. 
How will the results of the study be disseminated?

The results are expected to be presented and/or published at scientific discussions and meetings, in journal and/or conference papers, as well as in the doctoral thesis of Mr. Gómez-Laberge within the Faculty of Engineering at Carleton University, Ottawa, Canada. All results will be presented in a manner that will make identification of your information not possible.

How will the anonymity and confidentiality of my data be protected?

Your data will be completely anonymized by replacing any identifying information with a study code. Only the principal investigator will have access to the master file that links your name to your study code. Your anonymized data and the master file will be kept for fifteen (15) years at the Ottawa Hospital under lock-and-key before being destroyed. These data will not be shared; however, they may be viewed for audit purposes, under supervision of the principal investigator or his staff, by the Ottawa Hospital Research Institute and the Ottawa Hospital Research Ethics Board. We will collect your contact information so that we may contact you if an abnormality in the MRI scan is discovered.

\section{How are my legal rights protected?}

In the event of research-related side effects or injury, you will be provided with appropriate medical treatment/care. You are not waiving your legal rights by agreeing to participate in this study. The investigators and the hospital still have their legal and professional responsibilities.

\section{How and when can I withdraw myself from this study?}

You can withdraw your participation in this study at any time by informing any of the investigators either in person or by phone. This will not impact your care at the Ottawa Hospital now, or anytime in the future.

\section{Whom may I contact should I have questions or concerns?}

The investigators of this study are available to answer any questions you may have regarding this study. You may reach Dr. Matthew Hogan at the Ottawa Hospital at 613$798-5555 \times 16251$.

If you want any further information regarding your rights as a subject participating in a research study, or in the event of a research related injury, you may contact the Chair of the Ottawa Hospital Research Ethics Board at 613-798-5555 x 14902. 
Informed Consent Signature Sheet

Optimization of an event-related fMRI paradigm

using a visual feedback controlled motor task

A pilot study to determine the diagnostic potential of functional MRI for identifying cerebrovascular reactivity and interconnectivity of responding regions

Principal Investigator: Dr. Matthew J. Hogan

Participant's agreement to participate in this study:

My signature below indicates that I have read all four pages of information about this study, including the research procedure, possible risks, and potential benefits to me. The content and the meaning of this information have been explained to me, and I understand it. I have had the opportunity to ask questions. I agree to participate in the study described above.

I will be given a copy of this signed information sheet. I have been informed that the purpose of this study is for the assessment of the diagnostic value of magnetic resonance imaging of brain activity in a healthy population. I can withdraw from the study at any time without any negative consequences.

The research data from this study will remain confidential. By signing this form, I have not waived any of my rights. It simply indicates that I have agreed to participate in this study.

Participant's Name in Print

Participant's Signature

Date

Investigator's statement:

My signature below indicates that I have witnessed the participant read all four pages of information and have answered all of the participant's questions clearly, truthfully, and to their satisfaction.

Investigator's / Delegate's Name in Print 


\section{Participant Questionnaire Form}

\section{Optimization of an event-related fMRI paradigm}

using a visual feedback controlled motor task

A pilot study to determine the diagnostic potential of functional MRI for identifying cerebrovascular reactivity and interconnectivity of responding regions

Principal Investigator: Dr. Matthew J. Hogan

STUDY CODE: - 01 - EFMRI01

Please read and complete the information below carefully and ask questions about anything you don't understand in this form.

1. Please complete: Age:

Gender:

2. Are you right- or left-handed?

$\square$ RIGHT , $\square$ LEFT , $\square$ BOTH

3. Have you had prior surgery or an operation of any kind?

$\square$ YES or $\square$ NO

If yes, please list procedure name, date, and location:

4. Have you had a prior MRI examination?

$\square$ YES or $\square$ NO

If yes, please list body part imaged, date, and location:

5. Have you experienced any problem related to a previous MRI examination?

$\square$ YES or $\square$ NO

If yes, please describe:

6. Have you had an injury to the eye involving a metallic object or fragment (e.g., metallic slivers, shavings, foreign body, etc.)?

$\square$ YES or $\square$ NO

If yes, please describe: 
7. Have you ever been injured by a metallic object or foreign body (e.g., BB, bullet, shrapnel, etc.)?

$\square$ YES or $\square$ NO

If yes, please describe:

8. Are you currently taking or have you recently taken any medication or drug?

$\square$ YES or $\square$ NO

If yes, please list:

9. Have you ever had a heart attack or stroke?

YES or $\square$ NO

10. Are you suffering from any diseases?

$\square$ YES or $\square$ NO

If yes, please list:

For female participants:

11. Are you pregnant or experiencing a late menstrual period?

$\square$ YES or $\square$ NO 


\section{Optimization of an event-related fMRI paradigm \\ using a visual feedback controlled motor task \\ Principal Investigator: Dr. Matthew J. Hogan}

\section{Summary}

Functional magnetic resonance imaging (fMRI) represents a non-invasive method to map brain activity and connectivity in response to specific motor, sensory or cognitive tasks. We propose to use BOLD (blood oxygen level dependent) fMRI to evaluate the pattern of brain activity in response to a brief motor task. The relationship between the BOLD response and the underlying neural activity is complex and incompletely understood. This response can be affected by both physiological factors (such as age, alertness, training) and by disease states (such as cerebrovascular disease). In order to use any specific fMRI paradigm to evaluate brain function in disease a clear understanding of the response in normal controls is required. The investigators have implemented a feedback controlled brief motor task that permits accurate control and monitoring of the physiological response. The optimal parameters for this task have not been determined nor has any training effect been evaluated in repeated studies. To use this task to monitor recovery from neurological illness an understanding of these issues is required. With the tight control and measurement of physiological response afforded by this paradigm evaluation of the process of coupling the neural activity to the BOLD response may be possible in this event related design. Analyses of these data will proceed both along standard lines using Statistical Parametric Mapping (SPM) and through use of a novel data driven analysis approach that has been established within the laboratory of the principal investigator.

\section{Experimental Procedure}

All fMRI procedures closely follow the protocol used in the GIST study currently approved by the OHREB and have been used in subjects enrolled into this study at The Ottawa Hospital.

\section{fMRI Task Training}

Study participants will undergo a training session lasting 15 minutes prior to entering the MRI scanner. This session will simulate the fMRI stimulation paradigm and expected response. Subjects will sit with soft compressible balls (Jackson-Pratt drains) placed in each hand. These pressure balls will be connected to pressure transducers that will generate an electrical signal in response to compression of the balls.

The first step will be to measure the maximum force that can be applied to the pressure balls in each hand (maximal applied force - MAF). Based on our experience with the GIST protocol, which utilizes a similar fMRI paradigm, this measurement will take 3 minutes. Once the maximal force is obtained all subsequent force targets and responses can be calibrated as a percentage of the MAF.

The training stage follows the MAF measurement. Subjects will view a display screen that will show two horizontal bars. One bar moves from a rest position (the bottom of the screen) to a stimulus position (which is $60 \%$ of the full screen height) for a short period (typically 2 seconds) and then returns to the rest position. The subject controls the position of the other bar and will squeeze the pressure ball to match the position of the stimulus bar as rapidly and accurately as possible. The force required to match the bar is typically $25 \%$ of the MAF and will be adjusted depending on the outcome of part 1 of this study. The speed and accuracy that the stimulus bar can be followed will be measured and calculated in real time to signal when training targets are 
achieved. Based on our own experience developing this paradigm for the GIST trial, training can be accomplished in 10 minutes in normal subjects.

\section{Magnetic Resonance Imaging}

Standard operating procedures will be followed for all MRI studies which will be performed on the 1.5 T MR scanner at the General Site of the Ottawa Hospital. Participants will be asked to lie supine with their head placed in the standard head frame which will be equipped with a mirror through which the subject can visualize the stimulus display screen. Subjects will wear a disposable set of nasal prongs that will be used to monitor end tidal $\mathrm{CO} 2$ using a portable capnometer during the study. Blood pressure and heart rate will be measured at the start and end of study. Pressure balls will be placed in each hand and the subject will be placed within the magnet. The operation of the stimulus equipment will then be tested for each hand. This setup phase will take 5 minutes. The fMRI imaging sequences will then be performed, as outlined below, with the imaging session typically lasting 35 minutes. Subjects will then be removed from the magnet.

During the study an emergency signalling device is available to the subject if they wish to be immediately removed from the scanner. The subject is also monitored by closed circuit TV during the study and will be removed at any indication of a problem. A study investigator will be present for all scans.

\section{Objectives}

\section{Optimal equipment positioning and illumination}

The investigators will determine the appropriate setup parameters of the stimulus equipment and scanning environment. In particular, the optimal position and height of the projection screen, and the most comfortable ambient lighting and graphical presentation parameters (color, brightness) will be recorded.

\section{Optimal target fraction of the maximal applied force (MAF)}

fMRI data collected from three subjects will be used to determine the optimal target force exerted on the plastic pressure balls to adequately respond to the stimulus. Along with an increase in the target fraction of MAF, we expect to observe an increase in fMRI activity as well as involuntary motion and subject discomfort.

\section{Optimal stimulus duration}

fMRI data collected from six subjects will be used to determine a suitable stimulus duration to produce a clear signal yet remain as brief as possible. We expect the response signal to deteriorate as the stimulus duration is decreased.

\section{Assessment of fMRI signal reproducibility}

Ten subjects will attend 2 visits, being at least 3 months apart, during which they will undergo six 5 minute fMRI scans preceded by the training session outlined above. fMRI as well as pressure transducer data will be recorded to assess the reproducibility of the image signal as well as determine whether a training effect exists between the two visits. We expect to observe a reproducible fMRI signal, localized in the sensorimotor cortex, in all scans. We will determine the variability in $\mathrm{fMRI}$ and physiological data and test the null hypothesis that no training effect exists. 


\section{Experimental Procedure}

\section{Part 1: Optimization of Stimulus Protocol Parameters}

\subsection{Equipment positioning and illumination}

The investigators will setup the visual stimulus equipment consisting of one standing MRI-compatible projection screen to be placed inside the scanner room, and one projector and laptop to be placed in the control room. The visual stimulus program will be projected through the observation window onto the projection screen.

\subsection{Target fraction of maximal applied force}

The visual stimulus equipment described in part 1.1 and the stimulus response equipment consisting of two plastic pressure balls with tubing placed inside the MR room, two pressure transducers placed in the instrumentation room, and trigger-pulse detection equipment placed in the control room. The subject's breath rate (BR) and endtidal $\mathrm{PCO}_{2}$ will be monitored via nasal prongs using capnometer placed inside the MR room at the 5 Gauss line.

The subject will undergo three 5 minute fMRI scans (using a standard EPI pulse sequence) during which they will respond to stimuli, with their dominant hand, with force targets set to $25 \%, 50 \%$, and $75 \%$ of the MAF. This will be immediately followed by a 5 minute anatomical scan (standard $\mathrm{T}_{2}$-FLAIR pulse sequence) during which the subject need only lie still. Finally, three fMRI studies as described above will be repeated. The stimulus duration will be set to 2.0 seconds for these scans.

The subject's blood pressure (BP) and heart rate (HR) will be measured before and after MRI using a manual sphygmomanometer. The three subjects who perform this task will also perform part 1.3; however, a minimum of 3 months will elapse before they are scanned again.

\subsection{Optimal stimulus duration}

The complete stimulus apparatus and capnometer will be setup, as described in parts 1.1 and 1.2. After calibration of the MAF and training, the subject will undergo three 5 minute fMRI scans, followed by a 5 minute anatomical scan, and then another battery three fMRI scans. The six fMRI scans will each have a distinct stimulus duration randomized from the set of $0.5,1.0,1.5,2.0,2.5$, and 3.0 second durations. The target force as a fraction of the MAF will be the optimal value determined in part 1.2. The subject will respond to all scans with the dominant hand. The subject's $B R, P C O_{2}, H R$, and BP will be recorded as in part 1.2.

\section{Part 2: Assessment of fMRI Signal Reproducibility}

The complete stimulus apparatus and capnometer will be setup, as described in parts 1.1 and 1.2. Before entering the scanner room, the MAF will be determined and the training session will be completed as described above (about 15 minutes duration). Subjects will undergo two 5 minute fMRI scans using optimal force and duration parameters. The response will be measured from the dominant hand in the first scan and the non-dominant hand in the second scan. Afterwards, a 5 minute anatomical scan will be completed, followed by two further 5 minute fMRI scans assessing the response from each hand. The subject's $\mathrm{BR}, \mathrm{PCO}_{2}, \mathrm{HR}$, and $\mathrm{BP}$ will be recorded as in part 1.2. Study subjects will return for a $2^{\text {nd }}$ fMRI scan after 3 months. 


\section{Data Analysis}

1. Image preprocessing

The SPM5 software package (Wellcome Trust Centre for Neuroimaging, University College of London, England) will be used to examine and preprocess the images. Movement artifacts will be mitigated by rigid body realignment, and the images will be spatially smoothed to improve the signal-to-noise ratio. These steps will result in a significant performance improvement of the following analyses at the cost of a tolerable lose of spatial resolution.

\section{Data-driven clustering}

After preprocessing, each session will be analyzed using an implementation of the fuzzy c-means clustering algorithm (Dunn 1974, Bezdek 1981) in the software package Evldent (Jarmasz, 2002) (National Research Council of Canada). The result is a partition of the brain into clusters whose member voxels have significantly correlated time sequences.

\section{Selection criteria for cluster activity}

The temporal and spatial structure of each cluster will be analyzed in relation to the paradigm sequence and the contiguity of the voxel set. We recently proposed cluster selection criteria for such analysis (Gomez-Laberge 2008). The result is a set of informative values for each cluster based on these criteria, which will be used in the selection of clusters indicative of response to the stimuli.

\section{Statistical inference on cluster activity}

A statistical framework will be applied to perform inference on the effective activation of clusters obtained from the fMRI session data. The statistical model will be a multivariate hierarchical model (Gelman 2004) that is fit using the selection criteria from part 3 for all clusters. For each cluster, the result is an estimate of the probability that the cluster voxels are activated by the paradigm stimulus.

\section{Scientific Validity}

Functional MRI is a non-invasive and potentially informative modality to observe brain function during the recovery process from brain insult. This is a pilot study designed to assess fMRI signal reproducibility under optimal conditions in healthy subjects using a novel visual feedback controlled motor task. An important corollary of this objective is the development of novel fMRI analysis methods based on modern statistical and pattern recognition techniques, which aim to reduce unnecessary assumptions about the data. This has the potential to extract regionally specific information on brain activation that may be overlooked in more classical model driven analyses methods but which may reveal important insights on brain function in disease states. 


\section{References}

Bezdek, J.C. (1981). Pattern Recognition with Fuzzy Objective Function Algorithms. Plenum Press, New York, USA.

Dunn, J.C. (1974). A fuzzy relative of the ISODATA process and its use in detecting compact wellseparated clusters. J. Cybern., vol. 3, pp. 32-57.

Gelman, A., Carlin, J.B., Stern, H.S., Rubin, D.B. (2004). Bayesian Data Analysis. Chapman \& Hall/CRC Press, Boca Raton, USA.

Gomez-Laberge, C., Adler, A., Cameron, I., Nguyen, T.B., Hogan, M.J. (2008). Selection criteria for the analysis of data-driven clusters in cerebral fMRI. IEEE Trans. Biomed. Eng., vol. 55., pp. 23722380.

Jarmasz, M., Somorjai, R.L. (2002). Exploring regions of interest with clustering analysis (EROICA) using a spectral peak statistic for selecting and testing the significance of fMRI activation time series. Artif. Intell. Med., vol. 25, pp. 45-67. 


\section{Case Report Form}

Optimization of an event-related fMRI paradigm

using a visual feedback controlled motor task

Principal Investigator: Dr. Matthew J. Hogan

Subject study code: - $01-$ EFMRI01

Investigator name:

Date of visit:

\section{LAST, FIRST}

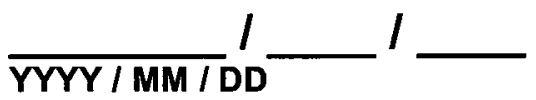

Location:

$\square$ General campus

$\square$ Civic campus

Scheduled procedure:

$\square$ Parts 1.2 and 1.3 (2 visits)

$\square$ Part 1.3 only (1 visit)

$\square$ Part 2 only (2 visits)

Obtained informed consent:

Obtained signed questionnaire:

Obtained signed MRI screening: 
Date:

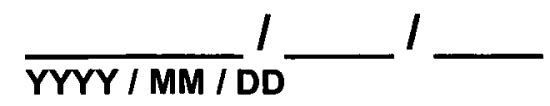

Time:

AM / PM

Equipment checklist

Consent forms:

$\square$ Signed consent \& questionnaire

$\square$ Signed MRI screen form

Stimulus apparatus:

$\square$ Laptop

$\square$ Pressure transducer cart

$\square$ Trigger pulse detector

$\square$ Squeeze balls and tubing

$\square$ Screen

$\square$ Projector

$\square$ Oscilloscope and test probes

$\square$ Blank CDs

Vitals:

$\square$ Capnometer and nasal prongs

$\square$ Sphygmomanometer stethoscope

$\square$ Pulse oximeter 
Scanner name / location:

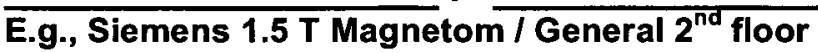

Functional MRI Parameters

Sequence directory:

$$
\text { E.g., TN > FMRI > GIST }
$$

Pulse sequence name:

$$
\text { E.g., ep2d_bold (epfid2d1_64) }
$$

Matrix size (interpolate?):

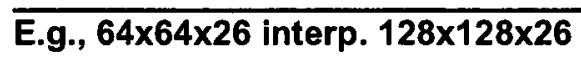

Voxel size $\left(\mathrm{mm}^{3}\right)$ :

$$
\text { E.g., } 1.7188 \times 1.7188 \times 5.0 \mathrm{~mm} 3
$$

$T_{R}(\mathrm{~ms})$ :

$T_{E}(\mathbf{m s}):$

Flip angle (deg.):

FFT scale factor $[0,1]$ :

\section{Anatomical MRI Parameters (T2-FLAIR) $\square$ Coregister with fMRI slices}

Sequence directory:

Pulse sequence name:

Matrix size (interpolate?):

Voxel size $\left(\mathrm{mm}^{3}\right)$ :

$T_{R}(m s):$

$T_{E}(\mathbf{m s}):$

Flip angle (deg.): 


\section{EXPERIMENTAL PROCEDURE: Part 1.2}

Setup:

$\square$ Stimulus apparatus in MR room

$\square$ Capnometer in MR room beyond 500 Gauss

Pre-MR room: $\square$ Did subject follow prep instructions? Y I N If no, explain:

$\square$ Is subject MR compatible now? Y / N No jewelry or metal

$\square$ BP (sys/dia mmHg) : I

MR room:

$\square$ HR (beats/min):

MR room:

Shimming/

Localizer:

Put on nasal prongs and ear plugs

Position subject in scanner $w /$ balls

$\square$ Make sure subject is in comfortable position

$\square$ Make sure subject sees screen clearly

fMRI 1:

$$
\text { Maximal applied force (MAF) calibration }
$$

Remind subject to lie still + focus on task

$\square$ Dominant hand L / R 25\% MAF

$\square 0$ min. $\quad$ PCO2 (mmHg):

BR:

$2.5 \mathrm{~min}$. PCO2 $(\mathrm{mmHg})$ : BR:

5 min. PCO2 $(\mathrm{mmHg})$ : BR: Comments: 
$\square 0$ min. $\quad$ PCO2 (mmHg): BR:

$\square 2.5$ min. PCO2 (mmHg): BR:

$\square 5$ min. $\quad$ PCO2 $(\mathrm{mmHg})$ : BR: Comments:

T2-FLAIR:

Dominant hand L / R $\quad \mathbf{7 5 \%}$ MAF

$\square 0$ min. $\quad$ PCO2 (mmHg): BR:

2.5 min. PCO2 $(\mathrm{mmHg})$ : BR:

$\square 5$ min. $\quad$ PCO2 (mmHg): BR: Comments:

fMRI 4:

$\square$ Dominant hand L / R 25\% MAF

$\square 0$ min. $\quad$ PCO2 (mmHg): BR:

$2.5 \mathrm{~min} . \mathrm{PCO} 2(\mathrm{mmHg})$ : BR:

5 min. PCO2 (mmHg): BR: Comments: 
$\square$ o min. PCO2 (mmHg): BR:

$\square 2.5$ min. PCO2 (mmHg): BR:

$\square 5$ min. $\quad$ PCO2 $(\mathrm{mmHg})$ : BR: Comments:

fMRI 6:

$\square$ Dominant hand L / R $\quad$ 75\% MAF

$\square 0$ min. $\quad$ PCO2 (mmHg): BR:

$2.5 \mathrm{~min}$. PCO2 $(\mathrm{mmHg})$ : BR:

$\square 5$ min. $\quad$ PCO2 $(\mathrm{mmHg})$ : BR: Comments:

Post-MR:

$\square$ BP (sys/dia mmHg) :

I

HR (beats/min):

$\square$ Did subject reclaim their metal items?

$\square$ Thank you very much!

Shutdown: $\square$ Start recording all data onto CDs

Stimulus apparatus

Capnometer

Time finished:

AM / PM 


\section{EXPERIMENTAL PROCEDURE: Part 1.3}

Setup:

$\square$ Stimulus apparatus in MR room

$\square$ Capnometer in MR room beyond 500 Gauss

Pre-MR room: $\square$ Did subject follow prep instructions? Y I N If no, explain:

$\square$ Is subject MR compatible now? Y / N No jewelry or metal

$\square$ BP (sys/dia mmHg) : I

$\square$ HR (beats/min):

MR room: $\quad \square$ Put on nasal prongs and ear plugs

$\square$ Position subject in scanner w/ balls

$\square$ Make sure subject is in comfortable position

$\square$ Make sure subject sees screen clearly

Shimming/ Localizer:

$\square$ Maximal applied force (MAF) calibration

fMRI 1:

Remind subject to lie still + focus on task

$\square$ Dominant hand L / R Duration:

$\square$ min. $\quad$ PCO2 (mmHg): BR:

$2.5 \mathrm{~min}$. PCO2 (mmHg): BR:

5 min. PCO2 $(\mathrm{mmHg})$ : BR:

Comments: 
fMRI 2:

$\square$ Dominant hand L / R Duration:

$\square 0$ min. PCO2 (mmHg): BR:

$\square 2.5 \mathrm{~min} . \quad \mathrm{PCO} 2(\mathrm{mmHg})$ : BR:

$\square 5$ min. $\quad$ PCO2 (mmHg): BR: Comments:

fMRI 3:

$\square$ Dominant hand L / R Duration:

$\square 0$ min. $\quad$ PCO2 $(\mathrm{mmHg})$ : BR:

$2.5 \mathrm{~min} . \quad \mathrm{PCO} 2(\mathrm{mmHg})$ : BR:

$\square 5$ min. PCO2 (mmHg): BR: Comments:

T2-FLAIR:

$\square$ Tell subject to rest but to keep lying still Comments:

fMRI 4:

$\square$ Dominant hand L / R Duration:

$\square 0$ min. PCO2 (mmHg): BR: $2.5 \mathrm{~min}$. PCO2 (mmHg): BR:

$\square$ min. PCO2 (mmHg): BR: Comments: 
fMRI 5:

$\square$ Dominant hand L/ R Duration:

$\square 0$ min. PCO2 (mmHg):

BR:

$\square 2.5 \mathrm{~min} . \quad$ PCO2 $(\mathrm{mmHg})$ :

BR:

$5 \mathrm{~min}$. PCO2 $(\mathrm{mmHg})$ :

BR:

Comments:

fMRI 6:

$\square$ Dominant hand L/R Duration:

$\square 0$ min. $\quad$ PCO2 (mmHg):

BR:

$\square 2.5 \mathrm{~min}$. PCO2 (mmHg):

BR:

$5 \mathrm{~min} . \quad \mathrm{PCO} 2(\mathrm{mmHg})$ :

BR:

Comments:

Post-MR:

BP (sys/dia mmHg) :

I

$\square$ HR (beats/min):

$\square$ Did subject reclaim their metal items?

$\square$ Thank you very much!

Shutdown: $\square$ Start recording all data onto CDs

$\square$ Stimulus apparatus

$\square$ Capnometer

Time finished:

AM / PM 


\section{EXPERIMENTAL PROCEDURE: Part 2 VISIT \#}

Setup:

$\square$ Stimulus apparatus in training room

$\square$ Capnometer in MR room beyond 500 Gauss

Training:

$\square$ Maximal applied force (MAF) calibration

$\square$ Training using both hands

$\square$ Setup stimulus apparatus in MR room

Pre-MR room: $\square$ Did subject follow prep instructions? Y I N If no, explain:

$\square$ Is subject MR compatible now? Y / N No jewelry or metal

$\square$ BP (sys/dia mmHg):

HR (beats/min):

MR room:

$\square$ Put on nasal prongs and ear plugs

$\square$ Position subject in scanner w/ balls

$\square$ Make sure subject is in comfortable position

Shimming/

Localizer:

$\square$ Remind subject to lie still + focus on task 
$\square 0$ min. $\quad$ PCO2 (mmHg):

BR:

$\square 2.5 \mathrm{~min} . \quad \mathrm{PCO} 2(\mathrm{mmHg})$ : BR:

$\square 5$ min. $\quad$ PCO2 $(\mathrm{mmHg})$ : BR: Comments:

fMRI 2:

Non-dominant hand L / R

$\square$ o min. $\quad$ PCO2 (mmHg): BR:

2.5 min. PCO2 $(\mathrm{mmHg})$ : BR:

$\square 5$ min. $\quad$ PCO2 $(\mathrm{mmHg})$ : BR: Comments:

T2-FLAIR:

$\square$ Tell subject to rest but to keep lying still Comments:

fMRI 3:

Dominant hand L / R

$\square 0$ min. $\quad$ PCO2 (mmHg):

BR:

$\square 2.5$ min. PCO2 (mmHg):

BR:

5 min. PCO2 $(\mathrm{mmHg})$ : BR: Comments: 
$\square 0$ min. PCO2 (mmHg): BR:

$\square 2.5 \mathrm{~min} . \quad$ PCO2 (mmHg): BR:

$\square 5$ min. $\quad$ PCO2 (mmHg): BR: Comments:

Post-MR:

$\square$ BP (sys/dia mmHg) : I

$\square$ HR (beats/min):

$\square$ Did subject reclaim their metal items?

$\square$ Thank you very much!

Shutdown: $\quad \square$ Start recording all data onto CDs

$\square$ Stimulus apparatus

$\square$ Capnometer

Time finished: AM / PM 


\section{Subject Master Record}

\section{Optimization of an event-related fMRI paradigm}

using a visual feedback controlled motor task

A pilot study to determine the diagnostic potential of functional MRI for identifying cerebrovascular reactivity and interconnectivity of responding regions

Principal Investigator: Dr. Matthew J. Hogan

This information is to be collected immediately after signing the consent form and is to be kept under lock-and-key by the principal investigator.

Subject study code: - 01 - EFMRI01

\section{Subject Identification:}

Name:

Address:

Phone:

E-mail: 